$\underline{\text { Supplementary Information }}$

\title{
Reversible Photoswitchable Inhibitors Generate Ultrasensitivity in Out-Of-Equilibrium Enzymatic Reactions
}

Michael Teders, Aleksandr A. Pogodaev, Glenn Bojanov, and Wilhelm T. S. Huck*

Institute for Molecules and Materials, Radboud University Nijmegen,

Heyendaalseweg 135, 6525 AJ, the Netherlands

w.huck@science.ru.nl 


\section{Table of Content}

1. GENERAL INFORMATION AND INSTRUMENTATION 5

2. SYNTHESIS AND CHARACTERISATION DATA OF PHOTOSWITCHABLE CHYMOTRYPSIN INHIBITORS (PIS, MAIN TEXT FIG. 2A) 9

2.1. Overview: Synthesized Photoswitchable $\alpha$-Chymotrypsin Inhibitors 9

2.2. Procedure for the Synthesis of most Pls 10

2.2.1. General Procedure for the Synthesis of Nitrosobenzenes (GP1) 10

2.2.2. General Procedure (GP2) for the Synthesis of Azobenzene Boronate Esters (25) 13

2.2.3. General Procedure (GP3) for the Synthesis of Potassium Trifluoroborate-Based PIs 20

$\begin{array}{ll}\text { 2.3. Synthesis of Potassium Trifluoroborate-Based PI } 3 & 27\end{array}$

2.3.1. Mills Reaction to Obtain 2-lodoazobenzene $57 \quad 27$

$\begin{array}{ll}\text { 2.3.2. Synthesis of Azobenzene Boronic Acid } \mathbf{5 8} & 28\end{array}$

2.3.3. Synthesis of Potassium Trifluoride-Based PI 3
28

2.4. Synthesis and Characterization Data of PI 15

2.4.1. Esterification of Benzoic Acid $\mathbf{5 0} \quad 29$

2.4.2. Synthesis of Potassium Trifluoride-Based PI 15 30

2.5. Synthesis of Potassium Trifluoroborate-Based PI 19 31

2.5.1. Mills Reaction to Obtain lodoazobenzene $\mathbf{5 9}$

2.5.2. Synthesis of Azobenzene Boronic Acid 60

2.5.3. Synthesis of Potassium Trifluoride-Based PI 19 32

3. PHOTOPHYSICAL CHARACTERIZATION OF PIS 33

3.1. UV/vis Absorption Studies of PIs $\quad 33$

3.2. Photoreversibility and -stability of PIs 41

3.3. Thermal Relaxation of cis-PIs $\quad 49$

3.4. Determination of cis-PI Isomer Content at $365 \mathrm{~nm}$ PSS $\quad 54$ 
$\begin{array}{ll}\text { 4.1. } & \text { Preparation of Stock Solutions }\end{array}$

$\begin{array}{lr}\text { 4.2. AMC Calibration } & 63\end{array}$

4.3. Michaelis-Menten Analysis of Cr/AAPF-AMC System 65

4.4. Determination of the Inhibition Constants of PIs 66

\begin{tabular}{ll} 
4.4.1. & General Procedure \\
\hline
\end{tabular}

$\begin{array}{lll}\text { 4.4.2. } & \text { Michaelis-Menten Plots and } K_{\mathrm{i}} \text { of PIs } & 67\end{array}$

$\begin{array}{ll}\text { 4.4.3. Overview of the Determined PI Inhibition Constants } & 78\end{array}$

4.5. Batch Photoswitching (Main Text Fig. 2B) 82

$\begin{array}{lr}\text { 4.6. Dose-Response-Curve } & 84\end{array}$

5. FLOW-EXPERIMENTS USING A CONTINUOUS-STIRRED FLOW TANK REACTOR 86

\begin{tabular}{ll} 
5.1. & General Information and Procedures \\
\hline
\end{tabular}

5.1.1. Preparation of Continuous-Stirred Flow Tank Reactor (CSTR) 86

5.1.2. Overview of the Setup Used for Flow Experiments $\quad 87$

$\begin{array}{llr}\text { 5.1.3. Preparation of Solutions for Flow Experiments } & 89\end{array}$

$\begin{array}{ll}\text { 5.1.4. Initiating the Flow Experiment } & 90\end{array}$

5.1.5. Conversion of Fluorescence Intensity to AMC Concentration 90

$\begin{array}{llr}\text { 5.1.6. Data Processing } & 91\end{array}$

5.2. Experimental Details and Results $\quad 92$

5.2.1. Multi-State Activity Control (Main Text Fig. 3C) 92

5.2.2. Alteration of Pulse Duration (Main Text Fig. 4A) 94

5.2.3. Alteration of Pulse Duration Cr/Ap System (Main Text Fig. 4C) 99

$\begin{array}{ll}\text { 5.2.4. } & \text { Alteration of Pulse Frequency (Main Text Fig. 5) } \\ \end{array}$

6. ARDUINO \& PYTHON SCRIPTS FOR CONTROLLING LEDS 109

7. MODEL SIMULATIONS OF THE ULTRASENSITIVE SYSTEM (MAIN TEXT FIG. 4B) 110

8. REFERENCES 112 
9. NMR SPECTRA

113 


\section{General Information and Instrumentation}

\section{Reaction set-up procedures and techniques}

The given reaction temperature corresponds to that of the oil bath or the alumina heating block surrounding the reaction tubes. Solid reagents or catalysts were added first to the reaction vessel followed by the addition of the solvent under standard counterflow protection gas working techniques. Afterwards, liquid reaction components as well as highly reactive (solid) starting materials or catalysts were added to the reaction vessel. Standard laboratory equipment and glassware was utilized for the performance of reactions. Following the described work-up and purification steps, the isolated products were evaporated at the rotary evaporator and potentially remaining solvent amounts were removed under high vacuum prior to product characterization. Hamilton gas-tight syringes or Eppendorf pipettes were used for transferring small amounts of liquids, while larger amounts were transferred via disposable syringes and cannulas.

\section{Chemicals}

Commercially available chemicals were obtained from Acros Organics, Alfa Aesar, Fischer Scientific, Fluorochem, Merck, Sigma Aldrich, TCI Europe or VWR and utilized without any further purification steps unless otherwise stated.

\section{Enzymes}

Chymotrypsin (bovine pancreas) was purchased from Sigma Aldrich:

- Chymotrypsin from bovine pancreas (Sigma Aldrich \#C4129), Type II, lyophilised powder, $\geq 40$ units $/ \mathrm{mg}$ protein. Purity $\geq 85 \%$ protein. $\mathrm{M}_{\mathrm{w}}=25.0 \mathrm{kDa}$.

For the calculation of enzyme concentration, Chymotrypsin was assumed to be essentially salt-free and calculations are based on the molecular weight specified above. Chymotrypsin was aliquoted as concentrated stock solution $(10$, or $100 \mu \mathrm{M})$, frozen in liquid nitrogen and kept at $-80^{\circ} \mathrm{C}$. Chymotrypsin aliquots were dissolved in a $\mathrm{HCl} / \mathrm{CaCl}_{2}(2 \mathrm{mM} / 20 \mathrm{mM})$ solution. Experiments were performed using a Tris/CaCl $/ \mathrm{BSA}_{2}$ buffer $(200 \mathrm{mM} / 20 \mathrm{mM} / 0.1 \mathrm{w}-\%)$ with a $\mathrm{pH} 7.8$ (adjusted with $1 \mathrm{M} \mathrm{HCl}, 99.99 \%$ purity).

\section{Solvents}

Solvents required for flash column chromatography, work-up, extraction or recrystallization (e.g. Acetone, $\mathrm{CH}_{2} \mathrm{Cl}_{2}, \mathrm{Et}_{2} \mathrm{O}$, EtOAc, n-pentane, THF, $\mathrm{MeOH}$ ) were supplied from Acros Organics, Merck, Sigma Aldrich or TCI Europe in the highest available quality and used without any further purification. Anhydrous DMF and $N, N$-Diisopropylethylamine (water content $<50 \mathrm{ppm}$ ) were purchased from Acros Organics or Sigma Aldrich and stored over molecular sieves under an atmosphere of argon.

\section{Thin layer chromatography}

Silica gel $\mathrm{F}_{254}$ aluminum sheets purchased from Merck were used for thin layer chromatography. Visualization of spots was performed by exposure to UV light $\left(\lambda_{\max }=254 \mathrm{~nm}\right)$ or by staining using cerium-ammonium-molybdate or potassium permanganate solutions followed by subsequent heating. 


\section{Flash column chromatography}

Silica gel (60 А, 0.035-0.070 mm), purchased from Acros Organics or Fluorochem, was used for column chromatography. Chromatographic purification was performed by employing a positive pressure of argon or pressurized air.

\section{Electrospray ionization mass spectrometry (ESI-MS)}

Mass spectra were obtained from a Thermo Scientific ${ }^{\mathrm{TM}}$ LCQ Fleet ${ }^{\mathrm{TM}}$ ion trap mass spectrometer with Gemini-NX C18 110A 150 × 2.0 mm column and JEOL Accurate Time of Flight (ToF) instruments, both using linear ion trap electrospray ionisation (ESI).

\section{UV/Vis absorption spectroscopy}

UV/Vis absorption spectra were recorded on a Jasco V-650 spectrophotometer, equipped with a temperature control thermostat. The samples were measured utilizing Hellma fluorescence QS quartz cuvettes (chamber volume $=1.4 \mathrm{~mL}, \mathrm{H} \times \mathrm{W} \times \mathrm{D}=46 \mathrm{~mm} \times 12.5 \mathrm{~mm} \times 12.5 \mathrm{~mm}$ ) sealed with a PTFE septum.

\section{Irradiation equipment - LEDs}

For irradiation, LEDs purchased from Avonec (www.avonec.de; $5 \mathrm{~W}, \lambda_{\max }=460 \mathrm{~nm} ; 3 \mathrm{~W}, \lambda_{\max }=$ $365 \mathrm{~nm}$ ) and powered by a Thorlabs LEDD1B driver were used. The emission spectra of the respective light sources are displayed below in Fig. S1.

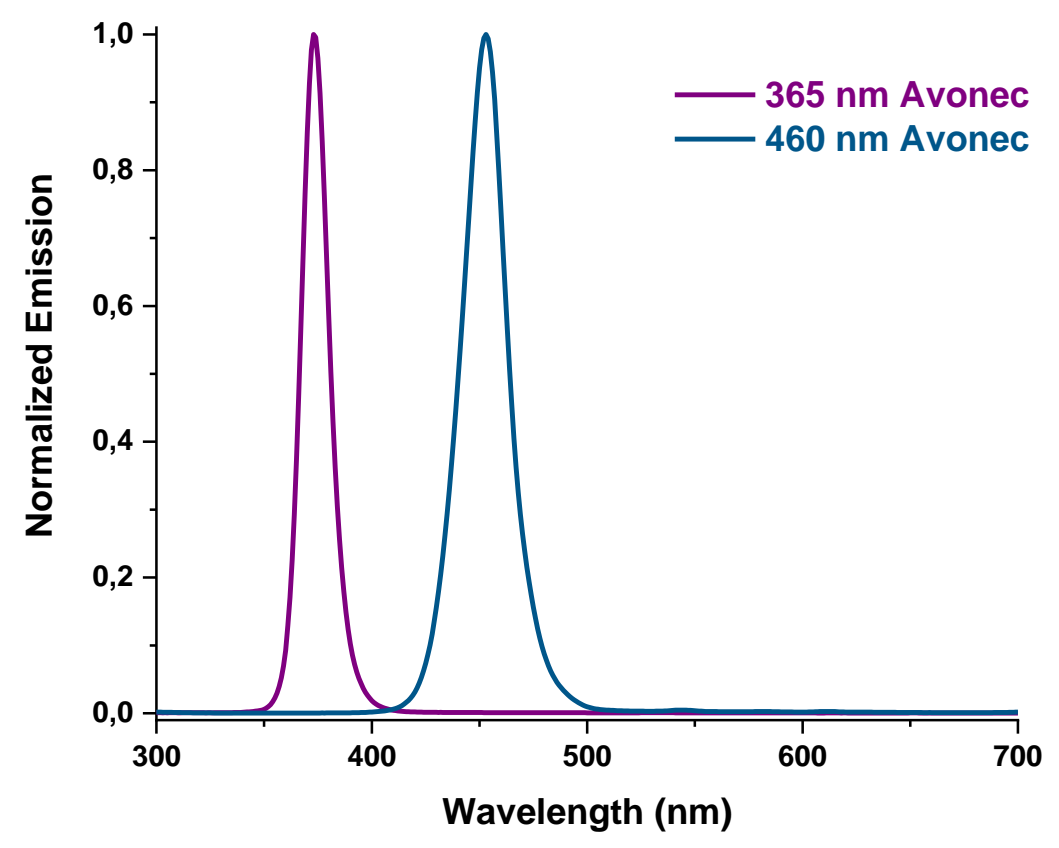

Figure S1. Emission spectra of the respective light sources used throughout the experiments. Displayed absorption data normalized based on the highest measured value. 


\section{NMR spectroscopy}

Unless otherwise noted, NMR spectra were measured at room temperature on a Bruker AVANCE III 400 spectrometer or a Varian Associates INOVA 400 spectrometer. The chemical shifts $(\delta)$ are given in ppm and ${ }^{1} \mathrm{H}$ and ${ }^{13} \mathrm{C}$ NMR spectra are reported relative to tetramethylsilane (TMS) by referencing the residual solvent signals (acetone- $d_{6}: \delta_{\mathrm{H}}=2.05 \mathrm{ppm}, \delta_{\mathrm{C}}=29.9 \mathrm{ppm} ; \mathrm{CD}_{2} \mathrm{Cl}_{2}: \delta_{\mathrm{H}}=5.32 \mathrm{ppm}$, $\delta_{\mathrm{C}}=54.0 \mathrm{ppm} ; \mathrm{CDCl}_{3}: \delta_{\mathrm{H}}=7.26 \mathrm{ppm}, \delta_{\mathrm{C}}=77.16 \mathrm{ppm} ; \mathrm{DMSO}-d_{6}: 2.50 \mathrm{ppm}, \delta_{\mathrm{C}}=39.52 \mathrm{ppm} ; \mathrm{D}_{2} \mathrm{O}$ : $\left.4.79 \mathrm{ppm} ; \mathrm{MeOH}-d: 3.31 \mathrm{ppm}, \delta_{\mathrm{C}}=49.00 \mathrm{ppm}\right) .{ }^{19} \mathrm{~F}$ NMR spectra are reported relative to $\mathrm{CCl}_{3} \mathrm{~F}$ as external standard and are thus not referenced using an internal standard. Reported multiplicities of NMR signals are reported according to the following abbreviations: $s$ (singlet), $d$ (doublet), $t$ (triplet), $q$ (quartet), quint (quintet), $\mathrm{m}$ (multiplet) or combinations of these. In the case of broad NMR signals, a " $\mathrm{b}$ " is added additionally in front of the multiplicity.

NOTE: Due to the trans/cis isomerization of compounds containing an azobenzene functionality, more signals were observed in both the ${ }^{1} \mathrm{H}$ and ${ }^{13} \mathrm{C}$ spectra than would be expected for the pure trans-isomer. Only signals for the major trans-isomer are reported.

\section{Flow experiments}

Cetoni Low Pressure High-Precision Syringe Pumps neMESYS 290N were used to carry out the flow experiments. Flow rates were configured using the Cetoni neMESYS microfluidic software. Production of fluorescent product in the continuously stirred tank reactor outflow was monitored using a self-made flow cuvette (see Fig. S2).

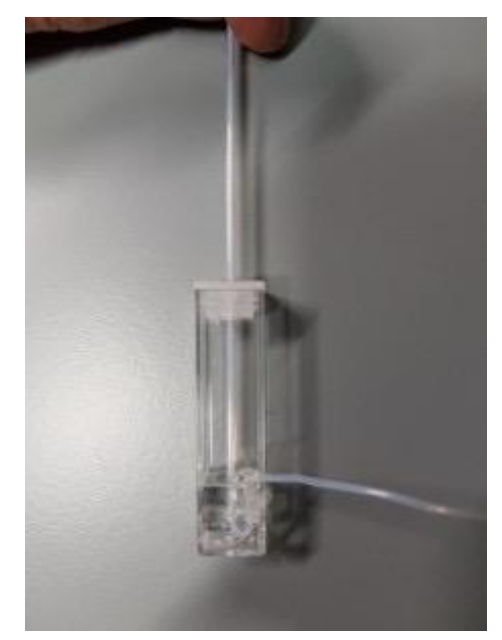

Figure S2. Picture of the self-made flow cuvette used for fluorescence readout during flow experiments.

The fluorescence readout was performed by using a AvaSpec-ULS2048L-EVO spectrometer, a AvaLight-LED 355/380 light source and fiber optic cables in combination with the AvaSoft-Basic Software - all purchased from Avantes.

\section{Fluorescence spectroscopy}

Fluorescence measurements, required for kinetic enzyme assays or inhibition constant determination, were performed with a Tecan Spark 10M plate reader equipped with a cryostat. Fluorescence intensity of wells within 96-well plates (purchased from Greiner Bio-One), containing $200 \mu \mathrm{L}$ of reaction mixture, was monitored for $40 \mathrm{sec}$ (shaking 3s/orbital mode/amplitude $4 \mathrm{~mm}$ ) at $25{ }^{\circ} \mathrm{C}$ using top fluorescence reading mode; $\lambda_{\mathrm{ex}} / \lambda_{\mathrm{em}}=380 \mathrm{~nm} / 460 \mathrm{~nm}$ for 7 -amino-4-methylcoumarin-based substrates. 
The autoinjector was used to add the enzyme stock solution to the respective well prior to orbital shaking and fluorescence analysis.

\section{Software}

Origin 2018b and Microsoft Excel were used for the fitting of rate and inhibition constants. SpectraGryph v. 1.2.14 was used for processing of UV/vis spectra. Python 3.6 with additionally installed tellurium package for simulation of kinetics has been used throughout the modelling studies.

\section{Optical bandpass filters}

In the course of UV-A irradiation of the PDMS reactor in flow experiments, a bandpass filter from Thorlabs with a center wavelength of $360 \mathrm{~nm}$ (FB360-10) was used (Full Width Half Max $=10 \pm 2 \mathrm{~nm}$ ). The filter was placed directly in front of the respective LED light source $(\sim 0.5 \mathrm{~cm}$ distance). 


\section{Synthesis and Characterisation Data of Photoswitchable Chymotrypsin Inhibitors (Cr-PIs, Main Text Fig. 2A)}

\subsection{Overview: Synthesized Photoswitchable $\alpha$-Chymotrypsin Inhibitors}
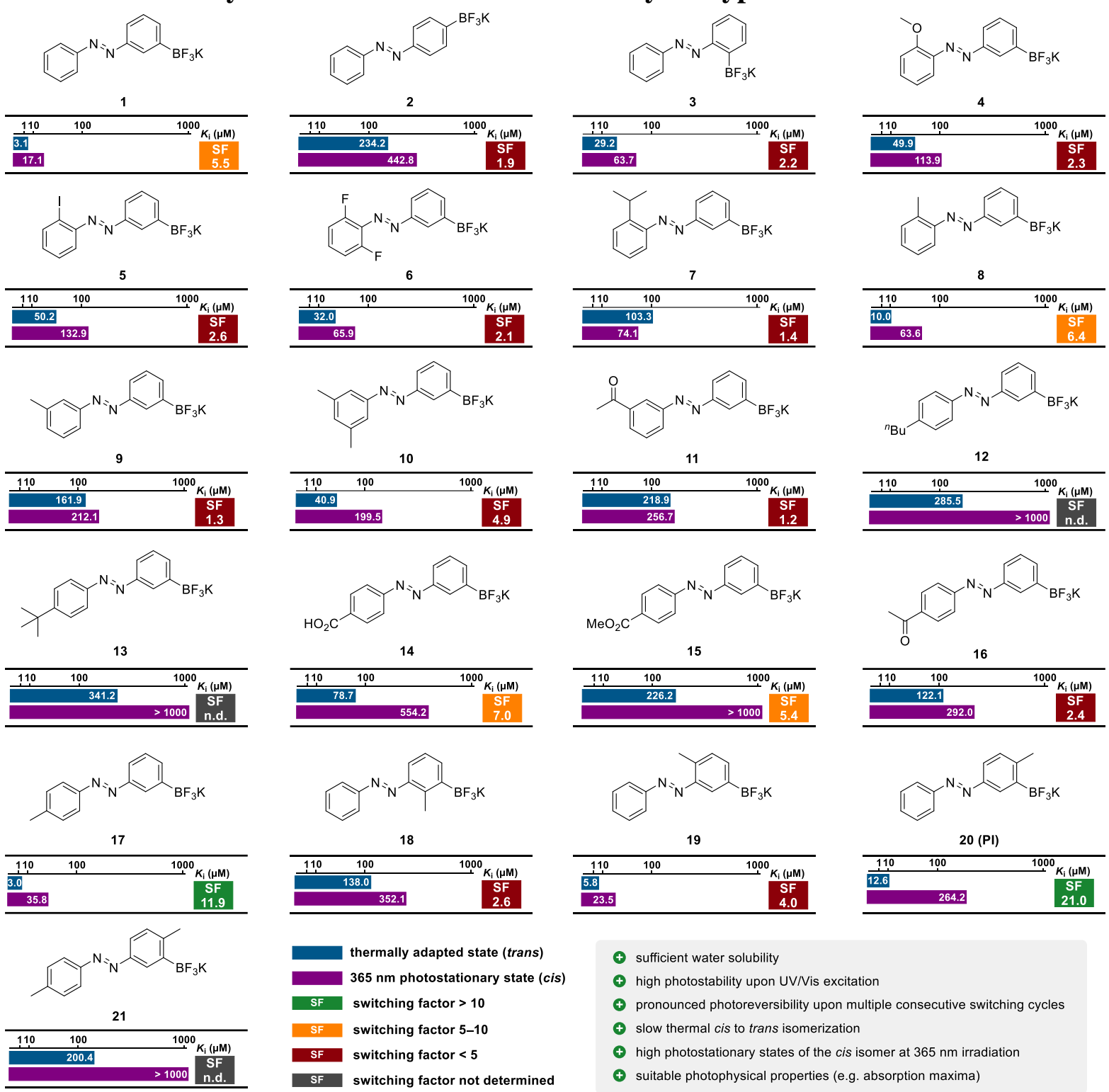

๑ sufficient water solubility

๑ high photostability upon UV/Nis excitation

๑ pronounced photoreversibility upon multiple consecutive switching cycles

(4) slow thermal cis to trans isomerization

( ) high photostationary states of the cis isomer at $365 \mathrm{~nm}$ irradiation

๑) suitable photophysical properties (e.g. absorption maxima)

Figure S3. Overview: Structures of photoswitchable chymotrypsin inhibitors synthesized throughout the structure-activityrelationship study. 


\subsection{Procedure for the Synthesis of Most PIs}

Photoswitchable chymotrypsin inhibitors 1-21 (PIs), except for 3, 15 and 19, were synthesized according to the 3-step procedure outlined in Fig. S4.

\section{1) Nitrosobenzene formation from corresponsing anilines using Oxone $\left(2 \mathrm{KHSO}_{5} \cdot \mathrm{KHSO}_{4} \cdot \mathrm{K}_{2} \mathrm{SO}_{4}\right)$}
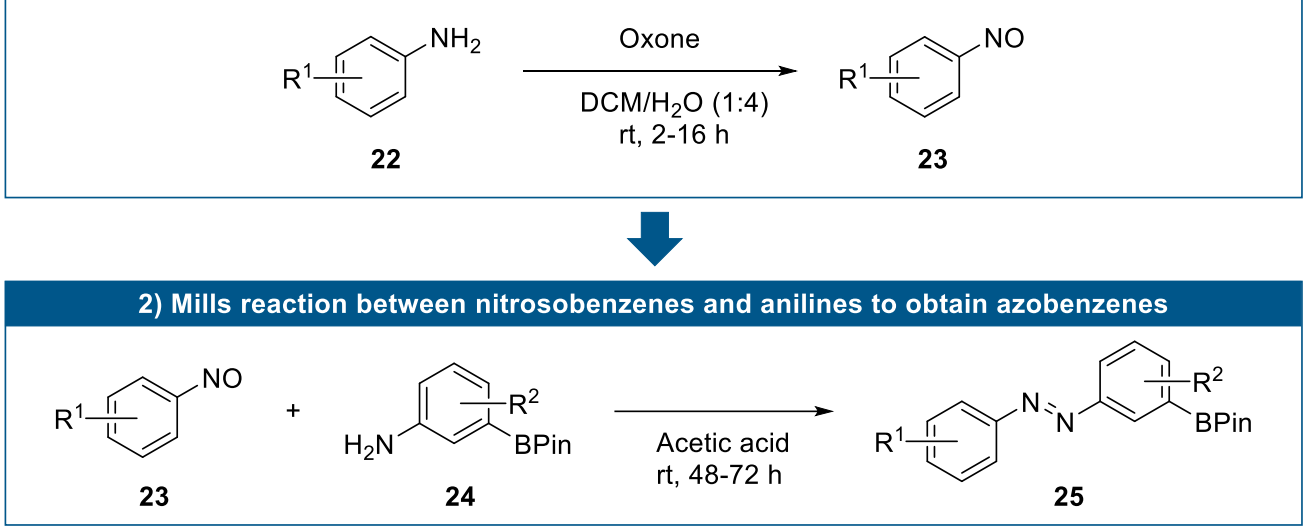

3) Synthesis of potassium trifluoroborate-based Pls using potassium bifluoride

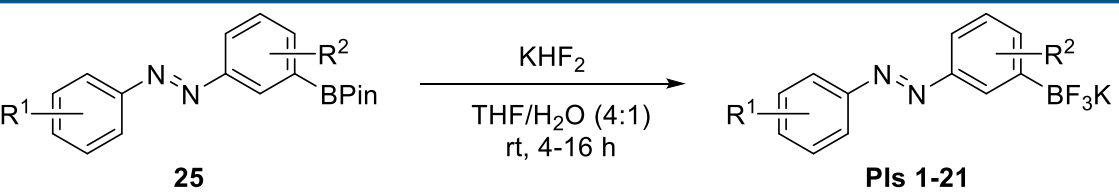

Figure S4. 3-Step procedure for the synthesis of most photoswitchable chymotrypsin inhibitors.

In the first step, the nitrosobenzenes $\mathbf{2 3}$ were obtained by oxidation of the corresponding anilines $\mathbf{2 2}$ using $\mathrm{OXONE}^{\circledR}$ as oxidant (see 2.2.1.). As some nitrosobenzenes were commercially available, this step did not have to be performed in the course of the synthesis of all PIs. The nitrosobenzenes $\mathbf{2 3}$ where then reacted with the corresponding aniline derivatives $\mathbf{2 4}$ in a Mills reaction to obtain the azobenzene boronic acid pinacol esters $\mathbf{2 5}$ (see 2.2.2.). The potassium trifluoroborate-based PIs 1-21 were obtained by reacting $\mathbf{2 5}$ with potassium bifluoride (see 2.2.3.).

\subsubsection{General Procedure for the Synthesis of Nitrosobenzenes (GP1)}

Nitrosobenzene formation from corresponding anilines using oxone $\left(2 \mathrm{KHSO}_{5} \cdot \mathrm{KHSO}_{4} \cdot \mathrm{K}_{2} \mathrm{SO}_{4}\right)$

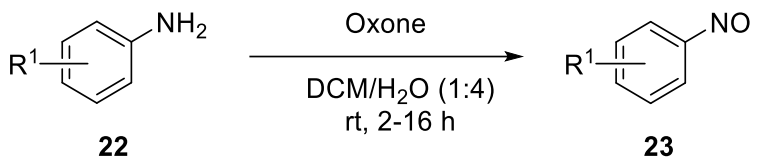

The nitrosobenzenes were synthesized following a modified literature report from Priewisch and RückBraun. ${ }^{1}$ A solution of Oxone ${ }^{\circledR}(10.0 \mathrm{mmol}, 2.0$ equiv) in MilliQ $(40 \mathrm{~mL})$ was added dropwise to a stirred solution of the corresponding aniline $22(5.0 \mathrm{mmol}, 1.0$ equiv) in DCM $(10 \mathrm{~mL})$ at room temperature. The resulting mixture was stirred vigorously at $\mathrm{rt}$ for $2-16 \mathrm{~h}$. Water $(50 \mathrm{~mL})$ was added and the layers were separated. The aqueous layer was extracted with DCM $(2 \times 50 \mathrm{~mL})$. The combined organic layers were washed with brine $(75 \mathrm{~mL})$, dried over anhydrous $\mathrm{MgSO}_{4}$, filtered and concentrated in vacuo. Due to the inherent instability of the synthesized nitrosobenzenes $\mathbf{2 3}$, they were used directly without further purification and characterization in the next step (GP2) as starting materials. 


\section{2-Methoxy-nitrosobenzene (26)}<smiles>COc1ccccc1N=O</smiles>

Prepared from ortho-anisidine $(565 \mu \mathrm{L}, 5.0 \mathrm{mmol})$ and Oxone ${ }^{\circledR}(3.074 \mathrm{~g}, 10.0 \mathrm{mmol})$ following general procedure GP1 (stirred at $\mathrm{rt}$ for $2 \mathrm{~h}$ ) to give the crude product $\mathbf{2 6}$ as dark red oil (372 mg, $2.71 \mathrm{mmol}, 55 \%)$.

\section{2-Iodo-nitrosobenzene (27)}<smiles>O=[N+]([O-])c1ccccc1I</smiles>

Prepared from 2-iodoaniline $(1.095 \mathrm{~g}, 5.0 \mathrm{mmol})$ and Oxone ${ }^{\circledR}(3.074 \mathrm{~g}, 10.0 \mathrm{mmol})$ following general procedure GP1 (stirred at $\mathrm{rt}$ for $8 \mathrm{~h}$ ) to give the crude product $\mathbf{2 7}$ as orange oil (862 mg, $3.71 \mathrm{mmol}, 74 \%)$.

\section{2,6-Difluoro-nitrosobenzene (28)}<smiles>O=[N+]([O-])c1ccccc1F</smiles>

Prepared from 2,6-difluoroaniline $(540 \mu \mathrm{L}, 5.0 \mathrm{mmol})$ and Oxone ${ }^{\circledR}(3.074 \mathrm{~g}, 10.0 \mathrm{mmol})$ following general procedure GP1 (stirred at $\mathrm{rt}$ for $4 \mathrm{~h}$ ) to give the crude product $\mathbf{2 8}$ as green oil (401 mg, $2.80 \mathrm{mmol}, 56 \%)$.

\section{2-Isopropyl-nitrosobenzene (29)}<smiles>CC(C)c1ccccc1N=O</smiles>

Prepared from 2-isopropylaniline $(700 \mu \mathrm{L}, 5.0 \mathrm{mmol})$ and Oxone ${ }^{\circledR}(3.074 \mathrm{~g}, 10.0 \mathrm{mmol})$ following general procedure GP1 (stirred at $\mathrm{rt}$ for $4 \mathrm{~h}$ ) to give the crude product $\mathbf{2 9}$ as brown oil (420 mg, $2.81 \mathrm{mmol}, 56 \%)$.

\section{1-Methyl-3-nitrosobenzene (30)}

NO Prepared from meta-toluidine $(535 \mathrm{mg}, 5.0 \mathrm{mmol})$ and Oxone ${ }^{\circledR}(3.074 \mathrm{~g}, 10.0 \mathrm{mmol})$ following general procedure GP1 (stirred at $\mathrm{rt}$ for $3 \mathrm{~h}$ ) to give the crude product $\mathbf{3 0}$ as brown oil (420 mg, $3.47 \mathrm{mmol}, 69 \%)$.

\section{1,3-Dimethyl-5-nitrosobenzene (31)}<smiles>Cc1cc(C)cc([N+](=O)[O-])c1</smiles>

Prepared from 3,5-Dimethylaniline $(627 \mu \mathrm{L}, 5.0 \mathrm{mmol})$ and Oxone ${ }^{\circledR}(3.074 \mathrm{~g}, 10.0 \mathrm{mmol})$ following general procedure GP1 (stirred at $\mathrm{rt}$ for $4 \mathrm{~h}$ ) to give the crude product $\mathbf{3 1}$ as green oil (412 mg, $3.05 \mathrm{mmol}, 61 \%)$.

\section{1-(3-Nitrosophenyl)ethan-1-one (32)}<smiles>CC(=O)c1cccc([N+](=O)[O-])c1</smiles>

Prepared from 3-acetylaniline $(676 \mathrm{mg}, 5.0 \mathrm{mmol})$ and Oxone ${ }^{\circledR}(3.074 \mathrm{~g}, 10.0 \mathrm{mmol})$ following general procedure GP1 (stirred at $\mathrm{rt}$ for $4 \mathrm{~h}$ ) to give the crude product $\mathbf{3 2}$ as brown oil (425 mg, $2.86 \mathrm{mmol}, 57 \%$ ). 


\section{1-Butyl-4-nitrosobenzene (33)}

(1)

Prepared from 4-butylaniline $(790 \mu \mathrm{L}, 5.0 \mathrm{mmol})$ and Oxone ${ }^{\circledR}(3.074 \mathrm{~g}, 10.0 \mathrm{mmol})$ following general procedure GP1 (stirred at $\mathrm{rt}$ for $6 \mathrm{~h}$ ) to give the crude product $\mathbf{3 3}$ as green oil (480 mg, $2.95 \mathrm{mmol}, 59 \%)$.

\section{1-tert-butyl-nitrosobenzene (34)}

1

Prepared from 4-tert-butylaniline $(796 \mu \mathrm{L}, 5.0 \mathrm{mmol})$ and Oxone ${ }^{\circledR}(3.074 \mathrm{~g}, 10.0 \mathrm{mmol})$ following general procedure GP1 (stirred at $\mathrm{rt}$ for $5 \mathrm{~h}$ ) to give the crude product $\mathbf{3 4}$ as green oil (460 mg, $2.82 \mathrm{mmol}, 56 \%)$.

\section{4-Nitrosobenzoic acid (35)}<smiles>O=C(O)c1ccc([N+](=O)[O-])cc1</smiles>

Prepared from 4-aminobenzoic acid $(686 \mathrm{mg}, 5.0 \mathrm{mmol})$ and Oxone ${ }^{\circledR}(3.074 \mathrm{~g}, 10.0 \mathrm{mmol})$ following general procedure GP1 (stirred at rt for $16 \mathrm{~h}$ ) to give the crude product $\mathbf{3 5}$ as beige solid (414 mg, $2.7 \mathrm{mmol}, 55 \%)$.

\section{1-(4-Nitrosophenyl)ethan-1-one (36)}

NO

Prepared from 4-acetylaniline $(676 \mathrm{mg}, 5.0 \mathrm{mmol})$ and Oxone ${ }^{\circledR}(3.074 \mathrm{~g}, 10.0 \mathrm{mmol})$ following general procedure GP1 (stirred at $\mathrm{rt}$ for $4 \mathrm{~h}$ ) to give the crude product $\mathbf{3 6}$ as black solid (530 mg, $3.56 \mathrm{mmol}, 71 \%$ ).

\section{1-Methyl-4-nitrosobenzene (37)}<smiles>Cc1ccc([N+](=O)[O-])cc1</smiles>

Prepared from para-toluidine $(536 \mathrm{mg}, 5.0 \mathrm{mmol})$ and Oxone ${ }^{\circledR}(3.074 \mathrm{~g}, 10.0 \mathrm{mmol})$ following general procedure GP1 (stirred at $\mathrm{rt}$ for $3 \mathrm{~h}$ ) to give the crude product $\mathbf{3 7}$ as green oil (290 mg, $2.4 \mathrm{mmol}, 48 \%)$. 
2.2.2. General Procedure (GP2) for the Synthesis of Azobenzene Boronate Esters (25)

Mills reaction between nitrosobenzenes and anilines to obtain azobenzene boronate esters

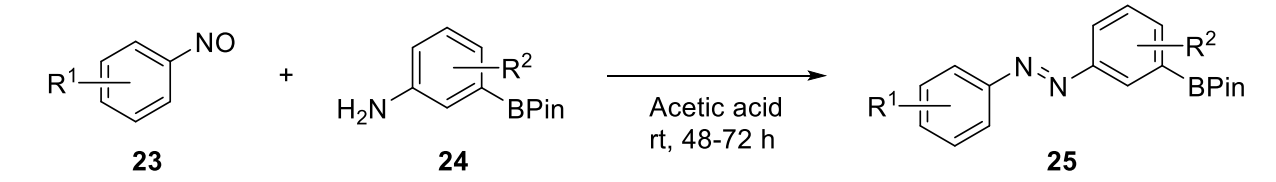

The azobenzene boronate esters $\mathbf{2 5}$ were synthesized following a literature report from Cheng et al. ${ }^{2}$ The respective nitrosobenzene $\mathbf{2 3}$ (1.0 equiv, crude from GP1 or commercially available) and the aniline boronate ester 24 (1.0 equiv) were dissolved in $\mathrm{AcOH}(50 \mathrm{~mL})$ and stirred at room temperature for 48 $72 \mathrm{~h}$. The solvent was removed and the residue was dissolved in DCM $(50 \mathrm{~mL})$. The solution was washed with saturated aq. $\mathrm{NaHCO}_{3}$ and brine, separately. The organic solution was dried over anhydrous $\mathrm{MgSO}_{4}$, filtered and concentrated in vacuo. The crude reaction products were purified by column chromatography over silica gel (eluent $=$ DCM or solvent gradient: eluent $=$ DCM $(100 \%)$ to DCM/methanol 95:5) to afford the pure azobenzene boronate esters $\mathbf{2 5}$.

\section{(E)-1-Phenyl-2-(3-(4,4,5,5-tetramethyl-1,3,2-dioxaborolan-2-yl)phenyl)diazene (38)}<smiles>CC1(C)OB(c2cccc(N=Nc3ccccc3)c2)OC1(C)C</smiles>

Prepared from nitrosobenzene $(321 \mathrm{mg}, 3.0 \mathrm{mmol})$ and 3-aminophenyl boronic acid pinacol ester $(657 \mathrm{mg}, 3.0 \mathrm{mmol})$ following general procedure GP2 (stirred at $\mathrm{rt}$ for $72 \mathrm{~h}$ ). Purification by column chromatography over silica gel (eluent $=100 \%$ DCM) afforded $\mathbf{3 8}$ as a red oil (393 $\mathrm{mg}, 1.28 \mathrm{mmol}$, $43 \%)$.

${ }^{1} \mathbf{H}$ NMR (400 MHz, CDCl $)$ ): $\delta 8.37(\mathrm{~s}, 1 \mathrm{H}), 8.01(\mathrm{ddd}, J=7.9,2.2,1.3 \mathrm{~Hz}, 1 \mathrm{H}), 7.98-7.85(\mathrm{~m}, 3 \mathrm{H})$, $7.56-7.45$ (m, 4H), 1.38 (s, 12H); ${ }^{13} \mathbf{C}\left\{{ }^{1} \mathbf{H}\right\}$ NMR (101 MHz, $\left.\mathbf{C D C l}_{3}\right): \delta 152.7,152.1,137.3,130.9$, $129.2,129.0,128.6,125.3,122.8,84.1,24.9$. The carbon directly attached to the boron atom was not detected due to quadrupolar broadening; ${ }^{11} \mathbf{B}$ NMR (128 $\left.\mathbf{M H z}, \mathbf{C D C l}_{\mathbf{3}}\right) \delta 32.3$ (bs); $\mathbf{R}_{\mathbf{f}}(\mathbf{D C M}): 0.75$.

\section{(E)-1-Phenyl-2-(4-(4,4,5,5-tetramethyl-1,3,2-dioxaborolan-2-yl)phenyl)diazene (39)}<smiles>CC1(C)OB(c2ccc(/N=N/c3ccccc3)cc2)OC1(C)C</smiles>

Prepared from nitrosobenzene $(321 \mathrm{mg}, 3.0 \mathrm{mmol})$ and 4-aminophenyl boronic acid pinacol ester $(657 \mathrm{mg}, 3.0 \mathrm{mmol})$ following general procedure GP2 (stirred at $\mathrm{rt}$ for $48 \mathrm{~h}$ ). Purification by column chromatography over silica gel (eluent $=100 \%$ DCM) afforded 39 as a red-orange oil $(480 \mathrm{mg}$, $1.56 \mathrm{mmol}, 52 \%)$.

${ }^{1} \mathbf{H}$ NMR (400 MHz, CDCl $\left.\mathbf{3}\right): \delta 8.00-7.87(\mathrm{~m}, 6 \mathrm{H}), 7.55-7.45(\mathrm{~m}, 3 \mathrm{H}), 1.38(\mathrm{~s}, 12 \mathrm{H}) ;{ }^{13} \mathbf{C}\left\{{ }^{1} \mathbf{H}\right\} \mathbf{~ N M R}$ (101 MHz, $\left.\mathbf{C D C l}_{\mathbf{3}}\right): \delta 154.1,152.6,135.9,131.0,129.2,123.3,121.9,84.1,24.9$. The carbon directly attached to the boron atom was not detected due to quadrupolar broadening; ${ }^{11} \mathbf{B}$ NMR (128 MHz, $\left.\mathbf{C D C l}_{3}\right) \delta 33.1(\mathrm{bs}) ; \mathbf{R}_{\mathbf{f}}(\mathbf{D C M}): 0.65$. 
<smiles>COc1ccccc1N=Nc1cccc(B2OC(C)(C)C(C)(C)O2)c1</smiles>

(10\% based on ${ }^{1} \mathrm{H}$ NMR analysis).

Prepared from 2-methoxy-nitrosobenzene $(371.0 \mathrm{mg}, 2.71 \mathrm{mmol})$ and 3aminophenyl boronic acid pinacol ester $(593 \mathrm{mg}, 2.71 \mathrm{mmol})$ following general procedure GP2 (stirred at rt for $48 \mathrm{~h}$ ). Purification by column chromatography over silica gel (eluent $=\mathrm{DCM}$ ) afforded $\mathbf{4 0}$ as a red solid (170.2 $\mathrm{mg}, 0.5 \mathrm{mmol}, 19 \%)$ along with an inseparable unknown impurity

${ }^{1} \mathbf{H}$ NMR (400 MHz, CDCl$): \delta 8.35(\mathrm{~s}, 1 \mathrm{H}), 8.08(\mathrm{dd}, J=8.0,1.7 \mathrm{~Hz}, 1 \mathrm{H}), 7.67-7.58(\mathrm{~m}, 2 \mathrm{H}), 7.50$ (ddd, $J=7.9,7.2,0.6 \mathrm{~Hz}, 1 \mathrm{H}), 7.45-7.37(\mathrm{~m}, 2 \mathrm{H}), 7.35-7.28(\mathrm{~m}, 1 \mathrm{H}), 4.01(\mathrm{~s}, 3 \mathrm{H}), 1.36(\mathrm{~s}, 12 \mathrm{H})$; ${ }^{13} \mathbf{C}\left\{{ }^{1} \mathbf{H}\right\}$ NMR (101 MHz, $\left.\mathbf{C D C l}_{3}\right): \delta 157.0,153.5,142.4,137.1,132.4,131.1,130.0,129.8,128.5$, $124.8,117.0,84.0,56.0,24.9$. The carbon directly attached to the boron atom was not detected due to quadrupolar broadening; ${ }^{11} \mathbf{B}$ NMR (128 MHz, $\left.\mathbf{C D C l}_{\mathbf{3}}\right) \delta 31.6$ (bs); $\mathbf{R}_{\mathbf{f}}(\mathbf{D C M}): 0.47$.

\section{(E)-1-(2-Iodophenyl)-2-(3-(4,4,5,5-tetramethyl-1,3,2-dioxaborolan-2-yl)phenyl)diazene (41)}

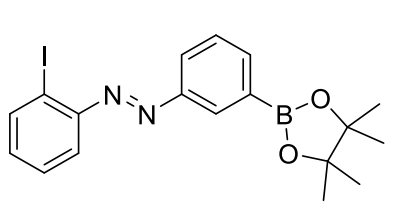

Prepared from 2-iodo-nitrosobenzene $(860 \mathrm{mg}, 3.69 \mathrm{mmol})$ and 3aminophenyl boronic acid pinacol ester $(808 \mathrm{mg}, 3.69 \mathrm{mmol})$ following general procedure GP2 (stirred at rt for $48 \mathrm{~h}$ ). Purification by column chromatography over silica gel (eluent $=$ DCM) afforded $\mathbf{4 1}$ as an orange oil (240 mg, $0.56 \mathrm{mmol}, 15 \%)$.

${ }^{1}$ H NMR (400 MHz, CDCl $)$ ): $\delta 8.45(\mathrm{~s}, 1 \mathrm{H}), 8.08-8.00(\mathrm{~m}, 2 \mathrm{H}), 7.93(\mathrm{dt}, J=7.3,1.2 \mathrm{~Hz}, 1 \mathrm{H}), 7.61$ $(\mathrm{dd}, J=8.0,1.6 \mathrm{~Hz}, 1 \mathrm{H}), 7.52(\mathrm{ddd}, J=8.0,7.3,0.5 \mathrm{~Hz}, 1 \mathrm{H}), 7.42(\mathrm{ddd}, J=8.0,7.2,1.3 \mathrm{~Hz}, 1 \mathrm{H}), 7.16$ (ddd, $J=7.9,7.2,1.6 \mathrm{~Hz}, 1 \mathrm{H}), 1.38(\mathrm{~s}, 12 \mathrm{H}) ;{ }^{13} \mathbf{C}\left\{{ }^{1} \mathbf{H}\right\}$ NMR (101 MHz, $\left.\mathbf{C D C l}_{3}\right): \delta 153.1,151.5,138.4$, $137.2,131.9,130.3,129.9,128.3,126.9,124.8,115.2,82.7,24.2$. The carbon directly attached to the boron atom was not detected due to quadrupolar broadening; ${ }^{11} \mathbf{B} \mathbf{N M R}\left(\mathbf{1 2 8} \mathbf{M H z}, \mathbf{C D C l}_{3}\right) \delta 31.5$ (bs); $\mathbf{R}_{\mathbf{f}}(\mathbf{D C M}): 0.70$.

(E)-1-(2,6-Difluorophenyl)-2-(3-(4,4,5,5-tetramethyl-1,3,2-dioxaborolan-2-yl)phenyl)diazene (42)<smiles>CC1(C)OB(c2cccc(NNc3c(F)cccc3F)c2)OC1(C)C</smiles>

Prepared from 2,6-difluoro-nitrosobenzene $(590 \mathrm{mg}, 3.36 \mathrm{mmol})$ and 3aminophenyl boronic acid pinacol ester $(735 \mathrm{mg}, 3.36 \mathrm{mmol})$ following general procedure GP2 (stirred at $\mathrm{rt}$ for $56 \mathrm{~h}$ ). Purification by column chromatography over silica gel (eluent $=$ DCM) afforded $\mathbf{4 2}$ as an orange oil (390 mg, $1.13 \mathrm{mmol}, 34 \%$ ).

${ }^{1} \mathbf{H}$ NMR (400 MHz, $\left.\mathbf{C D C l}_{3}\right): \delta 8.39-8.34(\mathrm{~m}, 1 \mathrm{H}), 8.00(\mathrm{ddd}, J=8.0,2.1,1.3 \mathrm{~Hz}, 1 \mathrm{H}), 7.95(\mathrm{dt}, J=$ 7.3, $1.2 \mathrm{~Hz}, 1 \mathrm{H}), 7.53$ (ddd, $J=7.9,7.2,0.6 \mathrm{~Hz}, 1 \mathrm{H}), 7.37-7.27$ (m, 1H), $7.08-7.00(\mathrm{~m}, 2 \mathrm{H}), 1.37$ (s, $\left.{ }_{12 H}\right) ;{ }^{13} \mathbf{C}\left\{{ }^{1} \mathbf{H}\right\}$ NMR (101 MHz, $\left.\mathbf{C D C l}_{3}\right): \delta 157.1,157.0,154.5,154.4,152.7,138.2,130.2,130.1$, $129.8,128.6,124.9,112.6,112.4,84.1,24.9$. The carbon directly attached to the boron atom was not detected due to quadrupolar broadening; ${ }^{19} \mathbf{F}$ NMR $\left(377 \mathbf{~ M H z}, \mathbf{C D C l}_{3}\right) \delta-123.29(\mathrm{~d}, J=9.2 \mathrm{~Hz}) ;{ }^{11} \mathbf{B}$ NMR (128 MHz, CDCl $) \delta 31.9$ (bs); $\mathbf{R}_{\mathbf{f}}$ (DCM): 0.66 . 


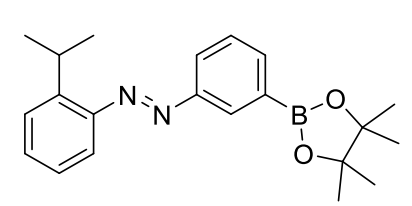

Prepared from 2-isopropyl-nitrosobenzene $(420 \mathrm{mg}, 2.86 \mathrm{mmol})$ and 3aminophenyl boronic acid pinacol ester $(625 \mathrm{mg}, 2.86 \mathrm{mmol})$ following general procedure GP2 (stirred at rt for $48 \mathrm{~h}$ ). Purification by column chromatography over silica gel (eluent $=\mathrm{DCM}$ ) afforded $\mathbf{4 3}$ as an orange solid (366 mg, $1.05 \mathrm{mmol}, 37 \%)$.

${ }^{1} \mathbf{H}$ NMR (400 MHz, CDCl $): \delta 8.38(\mathrm{~s}, 1 \mathrm{H}), 7.96(\mathrm{ddd}, J=8.0,2.1,1.2 \mathrm{~Hz}, 1 \mathrm{H}), 7.91(\mathrm{dt}, J=7.2,1.2$ $\mathrm{Hz}, 1 \mathrm{H}), 7.59$ (ddd, $J=8.1,1.4,0.6 \mathrm{~Hz}, 1 \mathrm{H}), 7.51$ (ddd, $J=7.8,7.2,0.5 \mathrm{~Hz}, 1 \mathrm{H}), 7.47-7.38(\mathrm{~m}, 2 \mathrm{H})$, $7.32-7.22(\mathrm{~m}, 1 \mathrm{H}), 4.13(\mathrm{p}, J=6.9 \mathrm{~Hz}, 1 \mathrm{H}), 1.38(\mathrm{~s}, 12 \mathrm{H}), 1.35(\mathrm{~s}, 3 \mathrm{H}), 1.33(\mathrm{~s}, 3 \mathrm{H}) ;{ }^{13} \mathbf{C}\left\{{ }^{1} \mathbf{H}\right\} \mathbf{~ N M R}$ (101 MHz, $\left.\mathbf{C D C l}_{3}\right): \delta$ 152.5, 149.8, 147.9, 137.1, 131.3, 131.1, 128.5, 126.3, 126.2, 123.5, 115.4, 84.1, 27.6, 24.9, 23.9. The carbon directly attached to the boron atom was not detected due to quadrupolar broadening; ${ }^{11} \mathbf{B}$ NMR (128 $\left.\mathbf{M H z}, \mathbf{C D C l}_{\mathbf{3}}\right) \delta 31.7$ (bs); $\mathbf{R}_{\mathbf{f}}$ (DCM): 0.81 .

\section{(E)-1-(3-(4,4,5,5-Tetramethyl-1,3,2-dioxaborolan-2-yl)phenyl)-2-(o-tolyl)diazene (44)}

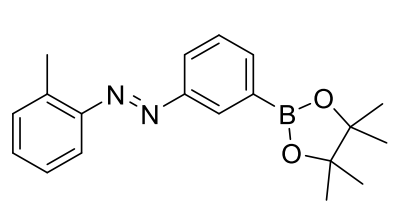

Prepared from 2-nitrosotoluene $(363.6 \mathrm{mg}, 3.0 \mathrm{mmol})$ and 3-aminophenyl boronic acid pinacol ester $(657 \mathrm{mg}, 3.0 \mathrm{mmol})$ following general procedure GP2 (stirred at $\mathrm{rt}$ for $48 \mathrm{~h}$ ). Purification by column chromatography over silica gel (eluent $=$ DCM) afforded 44 as a red oil $(411 \mathrm{mg}, 1.27 \mathrm{mmol}$, $43 \%)$.

${ }^{1} \mathbf{H}$ NMR (400 MHz, $\left.\mathbf{C D C l}_{3}\right): \delta 8.36(\mathrm{~s}, 1 \mathrm{H}), 7.98(\mathrm{ddd}, J=8.0,2.1,1.3 \mathrm{~Hz}, 1 \mathrm{H}), 7.90(\mathrm{dt}, J=7.3,1.2$ $\mathrm{Hz}, 1 \mathrm{H}), 7.65-7.59(\mathrm{~m}, 1 \mathrm{H}), 7.55-7.45(\mathrm{~m}, 1 \mathrm{H}), 7.39-7.21(\mathrm{~m}, 3 \mathrm{H}), 2.73(\mathrm{~s}, 3 \mathrm{H}), 1.37(\mathrm{~s}, 12 \mathrm{H})$; ${ }^{13} \mathbf{C}\left\{{ }^{1} \mathbf{H}\right\}$ NMR (101 MHz, $\left.\mathbf{C D C l}_{3}\right): \delta 152.4,150.9,138.0,137.1,131.2,130.8,130.4,128.5,126.4$, $124.4,115.6,84.0,24.9,17.6$. The carbon directly attached to the boron atom was not detected due to quadrupolar broadening; ${ }^{11} \mathbf{B} \mathbf{N M R}\left(\mathbf{1 2 8} \mathbf{~ M H z}, \mathbf{C D C l}_{\mathbf{3}}\right) \delta 31.5$ (bs); $\mathbf{R}_{\mathbf{f}}(\mathbf{D C M}): 0.81$.

(E)-1-(3-(4,4,5,5-tetramethyl-1,3,2-dioxaborolan-2-yl)phenyl)-2-(m-tolyl)diazene (45)

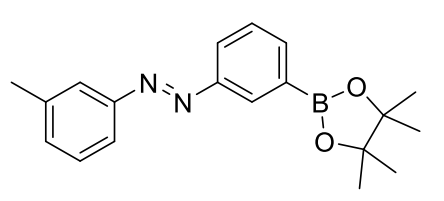

Prepared from 1-methyl-3-nitrosobenzene $(420 \mathrm{mg}, 3.0 \mathrm{mmol})$ and 3aminophenyl boronic acid pinacol ester $(670 \mathrm{mg}, 3.0 \mathrm{mmol})$ following general procedure GP2 (stirred at $\mathrm{rt}$ for $72 \mathrm{~h}$ ). Purification by column chromatography over silica gel (eluent $=$ DCM) afforded 45 as a red oil (574 mg, $1.78 \mathrm{mmol}, 59 \%$ ).

${ }^{1}$ H NMR (400 MHz, CDCl $)$ ): $\delta 8.36(\mathrm{~s}, 1 \mathrm{H}), 8.01(\mathrm{ddd}, J=7.9,2.2,1.3 \mathrm{~Hz}, 1 \mathrm{H}), 7.92(\mathrm{dt}, J=7.3,1.2$ $\mathrm{Hz}, 1 \mathrm{H}), 7.78-7.72(\mathrm{~m}, 2 \mathrm{H}), 7.53(\mathrm{t}, J=7.6 \mathrm{~Hz}, 1 \mathrm{H}), 7.41(\mathrm{dd}, J=8.4,7.5 \mathrm{~Hz}, 1 \mathrm{H}), 7.29$ (ddt, $J=7.5$, 1.9, $0.9 \mathrm{~Hz}, 1 \mathrm{H}), 2.49-2.45$ (s, 3H), $1.38(\mathrm{~s}, 12 \mathrm{H}) ;{ }^{\mathbf{1 3}} \mathbf{C}\left\{{ }^{1} \mathbf{H}\right\}$ NMR (101 MHz, $\left.\mathbf{C D C l}_{3}\right): \delta$ 152.8, 152.1, $137.2,131.7,129.2,128.9,128.5,125.3,122.9,120.5,84.0,24.9,21.34$. The carbon directly attached to the boron atom was not detected due to quadrupolar broadening; ${ }^{11} \mathbf{B}$ NMR $\left(\mathbf{1 2 8} \mathbf{~ M H z}, \mathbf{C D C l}_{\mathbf{3}}\right) \delta 31.4$ (bs); $\mathbf{R}_{\mathbf{f}}(\mathbf{D C M}): 0.8$. 
<smiles>Cc1cc(C)cc(N=Nc2cccc(B3OC(C)(C)C(C)(C)O3)c2)c1</smiles>

${ }^{1} \mathbf{H}$ NMR $\left(400 \mathrm{MHz}, \mathbf{C D C l}_{3}\right): \delta 8.34(\mathrm{~s}, 1 \mathrm{H}), 7.99(\mathrm{ddd}, J=7.9,2.2,1.3 \mathrm{~Hz}, 1 \mathrm{H}), 7.90(\mathrm{dt}, J=7.3,1.3$ $\mathrm{Hz}, 1 \mathrm{H}), 7.57-7.49(\mathrm{~m}, 3 \mathrm{H}), 7.14-7.11(\mathrm{~m}, 1 \mathrm{H}), 2.42(\mathrm{~s}, 6 \mathrm{H}), 1.38(\mathrm{~s}, 12 \mathrm{H}) ;{ }^{13} \mathbf{C}\left\{{ }^{1} \mathbf{H}\right\}$ NMR $(\mathbf{1 0 1}$ MHz, $\left.\mathbf{C D C l}_{3}\right): \delta 152.9,152.2,138.7,137.1,132.6,129.2,128.5,125.3,120.7,84.1,24.9,21.3$. The carbon directly attached to the boron atom was not detected due to quadrupolar broadening; ${ }^{11} \mathbf{B}$ NMR $\left(128 \mathbf{~ M H z}, \mathbf{C D C l}_{3}\right) \delta 31.8$ (bs); $\mathbf{R}_{\mathbf{f}}(\mathbf{D C M})$ : 0.75 .

\section{(E)-1-(3-((3-(4,4,5,5-tetramethyl-1,3,2-dioxaborolan-2-yl)phenyl)diazenyl)phenyl)ethan-1-one} (47)<smiles>CC(=O)c1cccc(/N=N/c2cccc(B3OC(C)(C)C(C)(C)O3)c2)c1</smiles>

Prepared from 1-(3-nitrosophenyl)ethan-1-one (425.1 mg, $2.86 \mathrm{mmol}$ ) and 3-aminophenyl boronic acid pinacol ester $(626.3 \mathrm{mg}, 2.86 \mathrm{mmol})$ following general procedure GP2 (stirred at rt for $48 \mathrm{~h}$ ). Purification by column chromatography over silica gel (eluent = DCM) afforded $\mathbf{4 7}$ as an orange oil (390 mg, $1.1 \mathrm{mmol}, 39 \%)$.

${ }^{1}$ H NMR (400 MHz, CDCl $): \delta 8.50-8.47(\mathrm{~m}, 1 \mathrm{H}), 8.40-8.37(\mathrm{~m}, 1 \mathrm{H}), 8.15-8.01(\mathrm{~m}, 3 \mathrm{H}), 7.94(\mathrm{dt}$, $J=7.3,1.3 \mathrm{~Hz}, 1 \mathrm{H}), 7.62(\mathrm{td}, J=7.8,0.5 \mathrm{~Hz}, 1 \mathrm{H}), 7.54(\mathrm{ddd}, J=7.8,7.2,0.6 \mathrm{~Hz}, 1 \mathrm{H}), 2.70(\mathrm{~s}, 3 \mathrm{H})$, 1.38 (s, 12H); ${ }^{13} \mathbf{C}\left\{{ }^{1} \mathbf{H}\right\}$ NMR (101 MHz, $\left.\mathbf{C D C l}_{3}\right): \delta$ 197.6, 152.7, 151.9, 138.1, 137.8, 130.1, 129.5, $129.4,128.7,127.0,125.4,122.9,84.1,26.8,24.9$. The carbon directly attached to the boron atom was not detected due to quadrupolar broadening; $\left.{ }^{11} \mathbf{B} \mathbf{~ N M R ~ ( 1 2 8 ~} \mathbf{~ M H z}, \mathbf{C D C l}_{\mathbf{3}}\right) \delta 31.7(\mathrm{bs}) ; \mathbf{R}_{\mathbf{f}}(\mathbf{D C M}): 0.62$.

\section{(E)-1-(4-Butylphenyl)-2-(3-(4,4,5,5-tetramethyl-1,3,2-dioxaborolan-2-yl)phenyl)diazene (48)}<smiles>CC1(C)OB(c2cccc(N=Nc3ccc(Br)cc3)c2)OC1(C)C</smiles>

Prepared from 1-butyl-4-nitrosobenzene $(480.0 \mathrm{mg}, 2.95 \mathrm{mmol})$ and 3aminophenyl boronic acid pinacol ester $(646 \mathrm{mg}, 2.95 \mathrm{mmol})$ following general procedure GP2 (stirred at $\mathrm{rt}$ for $48 \mathrm{~h}$ ). Purification by column chromatography over silica gel (eluent $=$ DCM) afforded $\mathbf{4 8}$ as a red oil (398 mg, $1.15 \mathrm{mmol}, 39 \%$ ).

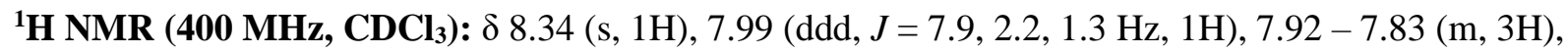
$7.52(\mathrm{ddd}, J=7.9,7.3,0.6 \mathrm{~Hz}, 1 \mathrm{H}), 7.35-7.29(\mathrm{~m}, 2 \mathrm{H}), 2.74-2.64(\mathrm{~m}, 2 \mathrm{H}), 1.71-1.61(\mathrm{~m}, 2 \mathrm{H}), 1.38$ $(\mathrm{s}, 12 \mathrm{H}), 0.95(\mathrm{t}, J=7.4 \mathrm{~Hz}, 3 \mathrm{H}) ;{ }^{13} \mathbf{C}\left\{{ }^{1} \mathbf{H}\right\} \mathbf{N M R}\left(\mathbf{1 0 1} \mathbf{~ M H z}, \mathbf{C D C l}_{3}\right): \delta 152.4,151.1,146.6,137.1$, $129.3,129.2,128.7,125.3,123.0,84.2,35.7,33.6,25.1,22.5,14.1$. The carbon directly attached to the boron atom was not detected due to quadrupolar broadening; ${ }^{11} \mathbf{B} \mathbf{N M R}\left(\mathbf{1 2 8} \mathbf{M H z}, \mathbf{C D C l}_{\mathbf{3}}\right) \delta 31.5$ (bs); $\mathbf{R}_{\mathbf{f}}(\mathbf{D C M}): 0.79$. 
<smiles>CC(C)(C)c1ccc(N=Nc2cccc(B3OC(C)(C)C(C)(C)O3)c2)cc1</smiles>

${ }^{1} \mathbf{H}$ NMR (400 MHz, CDCl $)$ ): $\delta 8.35(\mathrm{~s}, 1 \mathrm{H}), 7.99(\mathrm{ddd}, J=7.9,2.1,1.3 \mathrm{~Hz}, 1 \mathrm{H}), 7.94-7.84(\mathrm{~m}, 3 \mathrm{H})$, $7.58-7.49(\mathrm{~m}, 3 \mathrm{H}), 1.38(\mathrm{~s}, 21 \mathrm{H}) ;{ }^{13} \mathbf{C}\left\{{ }^{1} \mathbf{H}\right\} \mathbf{N M R}\left(\mathbf{1 0 1} \mathbf{M H z}, \mathbf{C D C l}_{3}\right): \delta 154.4,152.2,150.6,137.0$, $129.2,128.5,125.96,125.2,122.6,84.0,35.0,31.2,24.9$. The carbon directly attached to the boron atom was not detected due to quadrupolar broadening; $\left.{ }^{11} \mathbf{B ~ N M R ~ ( 1 2 8 ~} \mathbf{M H z}, \mathbf{C D C l}_{\mathbf{3}}\right) \delta 31.6$ (bs); $\mathbf{R}_{\mathbf{f}}(\mathbf{D C M})$ : 0.78 .

(E)-4-((3-(4,4,5,5-Tetramethyl-1,3,2-dioxaborolan-2-yl)phenyl)diazenyl)benzoic acid (50) aminophenyl boronic acid pinacol ester $(618 \mathrm{mg}, 2.82 \mathrm{mmol})$ following general procedure GP2 (stirred at $\mathrm{rt}$ for $72 \mathrm{~h}$ ). Purification by column chromatography over silica gel (eluent $=$ DCM) afforded 49 as an orange solid (483 mg, $1.25 \mathrm{mmol}, 44 \%)$.

Prepared from 1-tert-butyl-nitrosobenzene (460 mg, $2.82 \mathrm{mmol}$ ) and 3-

Prepared from 4-nitrosobenzoic acid (362.4 mg, $2.4 \mathrm{mmol})$ and 3-<smiles>CC1(C)OB(c2cccc(N=Nc3ccc(C(=O)O)cc3)c2)OC1(C)C</smilesaminophenyl boronic acid pinacol ester $(526 \mathrm{mg}, 2.4 \mathrm{mmol})$ following a modified general procedure GP2 (stirred at $\mathrm{rt}$ for $48 \mathrm{~h}$ ) with a $1 \mathrm{M} \mathrm{HCl}$ washing step instead of saturated aq. $\mathrm{NaHCO}_{3}$. Purification by column chromatography over silica gel (eluent $=$ $\mathrm{DCM} / \mathrm{MeOH}=95 / 5)$ afforded $\mathbf{5 0}$ as an orange solid (305 mg, $0.87 \mathrm{mmol}, 36 \%)$.

${ }^{1}$ H NMR (400 MHz, DMSO-d $\left.\mathbf{6}\right): \delta 8.20-8.13(\mathrm{~m}, 3 \mathrm{H}), 8.10$ (ddd, $\left.J=7.5,2.2,1.2 \mathrm{~Hz}, 1 \mathrm{H}\right), 8.03$ $7.98(\mathrm{~m}, 2 \mathrm{H}), 7.89(\mathrm{dt}, J=7.4,1.2 \mathrm{~Hz}, 1 \mathrm{H}), 7.67(\mathrm{t}, J=7.6 \mathrm{~Hz}, 1 \mathrm{H}), 1.34(\mathrm{~s}, 12 \mathrm{H}) ;{ }^{13} \mathbf{C}\left\{{ }^{1} \mathbf{H}\right\} \mathbf{N M R}$ (101 MHz, DMSO- $\left.\boldsymbol{d}_{6}\right): \delta 167.1,154.7,151.8,132.3,131.1,129.9,129.6,127.7,127.4,123.1,84.6$, 25.2. The carbon directly attached to the boron atom was not detected due to quadrupolar broadening; ${ }^{11} \mathbf{B}$ NMR (128 MHz, $\left.\mathbf{C D C l}_{3}\right) \delta 29.4(\mathrm{bs}) ; \mathbf{R}_{\mathbf{f}}(\mathbf{D C M} / \mathbf{M e O H}=95 / 5): 0.27$.

(E)-1-(4-((3-(4,4,5,5-tetramethyl-1,3,2-dioxaborolan-2-yl)phenyl)diazenyl)phenyl)ethan-1-one (51)<smiles>CC(=O)c1ccc(/N=N/c2cccc(B3OC(C)(C)C(C)(C)O3)c2)cc1</smiles>

Prepared from 1-(4-nitrosophenyl)ethan-1-one (577 mg, $4.27 \mathrm{mmol})$ and 3-aminophenyl boronic acid pinacol ester (935 $\mathrm{mg}, 4.27 \mathrm{mmol}$ ) following general procedure GP2 (stirred at rt for $48 \mathrm{~h}$ ). Purification by column chromatography over silica gel (eluent $=$ DCM) afforded $\mathbf{5 1}$ as an orange oil (419 mg, $1.2 \mathrm{mmol}, 28 \%$ ).

${ }^{1}$ H NMR (400 MHz, CDCl $)$ ): $\delta 8.40-8.37(\mathrm{~m}, 1 \mathrm{H}), 8.14-8.09(\mathrm{~m}, 2 \mathrm{H}), 8.03(\mathrm{ddd}, J=8.0,2.1,1.3$ $\mathrm{Hz}, 1 \mathrm{H}), 8.00-7.93(\mathrm{~m}, 3 \mathrm{H}), 7.55(\mathrm{ddd}, J=7.8,7.3,0.5 \mathrm{~Hz}, 1 \mathrm{H}), 2.67(\mathrm{~s}, 3 \mathrm{H}), 1.38(\mathrm{~s}, 12 \mathrm{H}) ;{ }^{13} \mathbf{C}\left\{{ }^{1} \mathbf{H}\right\}$ NMR (101 MHz, CDCl $)$ : $\delta 197.5,155.1,152.0,138.3,138.0,129.6,129.4,128.7,125.6,122.9,84.16$, $26.9,24.9$. The carbon directly attached to the boron atom was not detected due to quadrupolar broadening; ${ }^{11}$ B NMR (128 MHz, $\left.\mathbf{C D C l}_{\mathbf{3}}\right) \delta 31.9$ (bs); $\mathbf{R}_{\mathbf{f}}$ (DCM): 0.65 . 
<smiles>Cc1ccc(/N=N/c2cccc(B3OC(C)(C)C(C)(C)O3)c2)cc1</smiles>

${ }^{1} \mathbf{H}$ NMR (400 MHz, $\left.\mathbf{C D C l}_{3}\right): \delta 8.34(\mathrm{~s}, 1 \mathrm{H}), 7.99(\mathrm{ddd}, J=7.9,2.2,1.3 \mathrm{~Hz}, 1 \mathrm{H}), 7.90(\mathrm{dt}, J=7.3,1.2$ $\mathrm{Hz}, 1 \mathrm{H}), 7.87-7.82(\mathrm{~m}, 2 \mathrm{H}), 7.52(\mathrm{t}, J=7.6 \mathrm{~Hz}, 1 \mathrm{H}), 7.34-7.29(\mathrm{~m}, 2 \mathrm{H}), 2.44(\mathrm{~s}, 3 \mathrm{H}), 1.38(\mathrm{~s}, 12 \mathrm{H})$; ${ }^{13} \mathbf{C}\left\{{ }^{1} \mathbf{H}\right\}$ NMR (101 MHz, $\left.\mathbf{C D C l}_{\mathbf{3}}\right): \delta$ 152.2, 150.81, 141.4, 137.0, 129.7, 129.1, 128.5, 125.2, 122.8, $84.0,24.9,21.5$. The carbon directly attached to the boron atom was not detected due to quadrupolar broadening; ${ }^{11} \mathbf{B}$ NMR (128 MHz, $\left.\mathbf{C D C l}_{\mathbf{3}}\right) \delta 31.6$ (bs); $\mathbf{R}_{\mathbf{f}}(\mathbf{D C M}): 0.74$.

\section{(E)-1-(2-methyl-3-(4,4,5,5-tetramethyl-1,3,2-dioxaborolan-2-yl)phenyl)-2-phenyldiazene (53)}<smiles>Cc1c(N=Nc2ccccc2)cccc1B1OC(C)(C)C(C)(C)O1</smiles>

Prepared from nitrosobenzene $(214.2 \mathrm{mg}, 2.0 \mathrm{mmol})$ and 2-methyl-3aminophenyl boronic acid pinacol ester $(466.2 \mathrm{mg}, 2.0 \mathrm{mmol})$ following general procedure GP2 (stirred at $\mathrm{rt}$ for $72 \mathrm{~h}$ ). Purification by column chromatography over silica gel (eluent = DCM) afforded $\mathbf{5 3}$ as a red oil (310 mg, $0.97 \mathrm{mmol}, 48 \%$ ).

${ }^{1}$ H NMR (400 MHz, CDCl $): \delta 7.95-7.92(\mathrm{~m}, 2 \mathrm{H}), 7.89(\mathrm{dd}, J=7.3,1.5 \mathrm{~Hz}, 1 \mathrm{H}), 7.69(\mathrm{dd}, J=8.0$, $1.5 \mathrm{~Hz}, 1 \mathrm{H}), 7.55-7.43(\mathrm{~m}, 3 \mathrm{H}), 7.30-7.24(\mathrm{~m}, 1 \mathrm{H}), 2.94(\mathrm{~s}, 3 \mathrm{H}), 1.40(\mathrm{~s}, 12 \mathrm{H}) ;{ }^{13} \mathbf{C}\left\{{ }^{1} \mathbf{H}\right\} \mathbf{N M R}(\mathbf{1 0 1}$ MHz, $\left.\mathbf{C D C l}_{3}\right): \delta 153.0,150.8,144.5,138.7,130.7,129.0,125.6,123.0,118.0,83.7,24.9,16.2$. The carbon directly attached to the boron atom was not detected due to quadrupolar broadening; ${ }^{11} \mathbf{B}$ NMR $\left(\mathbf{1 2 8} \mathbf{~ M H z}, \mathbf{C D C l}_{\mathbf{3}}\right) \delta 31.9$ (bs); $\mathbf{R}_{\mathbf{f}}(\mathbf{D C M}): 0.81$.

\section{(E)-1-(4-methyl-3-(4,4,5,5-tetramethyl-1,3,2-dioxaborolan-2-yl)phenyl)-2-phenyldiazene (54)}

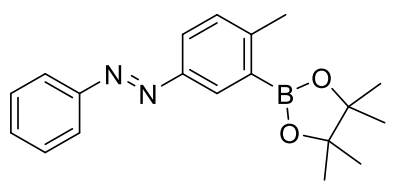

Prepared from nitrosobenzene $(160.6 \mathrm{mg}, 1.5 \mathrm{mmol})$ and 4-methyl-3aminophenyl boronic acid pinacol ester $(349.6 \mathrm{mg}, 1.5 \mathrm{mmol})$ following general procedure GP2 (stirred at $\mathrm{rt}$ for $48 \mathrm{~h}$ ). Purification by column chromatography over silica gel (eluent $=$ DCM) afforded $\mathbf{5 4}$ as an orange solid (370 mg, $1.15 \mathrm{mmol}, 76 \%)$.

${ }^{1} \mathbf{H}$ NMR (400 MHz, CDCl $): \delta 8.35(\mathrm{~d}, J=2.3 \mathrm{~Hz}, 1 \mathrm{H}), 7.95-7.90(\mathrm{~m}, 2 \mathrm{H}), 7.87(\mathrm{dd}, J=8.1,2.3 \mathrm{~Hz}$, $1 \mathrm{H}), 7.55-7.43(\mathrm{~m}, 3 \mathrm{H}), 7.31(\mathrm{dt}, J=8.1,0.7 \mathrm{~Hz}, 1 \mathrm{H}), 2.66-2.60(\mathrm{~m}, 3 \mathrm{H}), 1.38(\mathrm{~s}, 12 \mathrm{H}) ;{ }^{13} \mathbf{C}\left\{{ }^{1} \mathbf{H}\right\}$ NMR (101 MHz, $\left.\mathbf{C D C l}_{3}\right): \delta 151.1,150.3,138.4,135.9,131.0,130.5,124.7,124.2,123.4,84.6,26.8$, 22.1. The carbon directly attached to the boron atom was not detected due to quadrupolar broadening;

${ }^{11}$ B NMR (128 MHz, CDCl $\left.)_{3}\right) \delta 31.5$ (bs); $\mathbf{R}_{\mathbf{f}}(\mathbf{D C M}): 0.71$. 


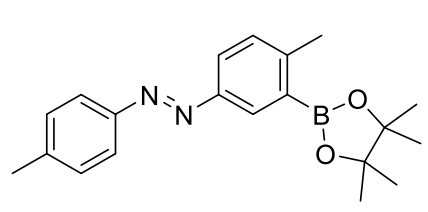

Prepared from 1-methyl-4-nitrosobenzene (290 mg, $2.4 \mathrm{mmol}$ ) and 2methyl-5-aminophenyl boronic acid pinacol ester (558 $\mathrm{mg}, 2.4 \mathrm{mmol}$ ) following general procedure GP2 (stirred at $\mathrm{rt}$ for $72 \mathrm{~h}$ ). Purification by column chromatography over silica gel (eluent $=$ DCM) afforded $\mathbf{5 5}$ as an orange oil (319 mg, $0.95 \mathrm{mmol}, 40 \%)$.

${ }^{1} \mathbf{H}$ NMR (400 MHz, $\left.\mathbf{C D C l}_{3}\right): \delta 8.33(\mathrm{~d}, J=2.3 \mathrm{~Hz}, 1 \mathrm{H}), 7.88-7.79(\mathrm{~m}, 3 \mathrm{H}), 7.37-7.26(\mathrm{~m}, 3 \mathrm{H}), 2.62$ (s, 3H), 2.43 (s, 3H), 1.38 (s, 12H); ${ }^{13} \mathbf{C}\left\{{ }^{1} \mathbf{H}\right\}$ NMR (101 $\left.\mathbf{~ M H z , ~} \mathbf{C D C l}_{3}\right): \delta 150.9,150.1,148.2,141.1$, $131.5,130.7,129.7,123.7,122.7,83.7,25.0,22.3,21.5$. The carbon directly attached to the boron atom was not detected due to quadrupolar broadening; $\left.{ }^{11} \mathbf{B} \mathbf{~ N M R ~ ( 1 2 8 ~} \mathbf{M H z}, \mathbf{C D C l}_{\mathbf{3}}\right) \delta 31.7$ (bs); $\mathbf{R}_{\mathbf{f}}(\mathbf{D C M})$ : 0.81 . 


\subsubsection{General Procedure (GP3) for the Synthesis of Potassium Trifluoroborate-Based}

PIs Synthesis of potassium trifluoroborate-based PIs from azobenzene boronate esters

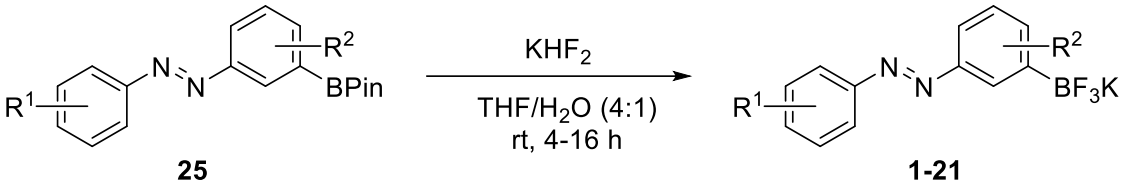

Azobenzene potassium trifluoroborates 1-21 were synthesized following a literature report from Westphal et al. ${ }^{3}$ In a polyethylene round-bottom flask, the azobenzene boronate ester $\mathbf{2 5}$ (1.0 equiv) was dissolved in a 4:1 mixture of THF and water $(10 \mathrm{~mL})$ and potassium bifluoride (3.0 equiv) was added. After stirring at room temperature for $4-16 \mathrm{~h}$ (TLC indicated full consumption of starting material), $\mathrm{NaHCO}_{3}$ (1.5 equiv) was added and the mixture was stirred for $10 \mathrm{~min}$. The volatiles were removed, the resulting oil or solid was extracted with acetone $(20 \mathrm{~mL})$ and the extract was filtered. The solution was concentrated in vacuo. Pure potassium trifluoroborate-based PIs 1-21 were obtained by recrystallisation from $\mathrm{DCM} / n$-pentane, $\mathrm{DCM} / \mathrm{Et}_{2} \mathrm{O}$ or $\mathrm{Et}_{2} \mathrm{O} / n$-pentane (see respective compound for used recrystallisation solvent system.

\section{(E)-1-phenyl-2-(3-(trifluoro-14-boraneyl)phenyl)diazene, potassium salt (1)}

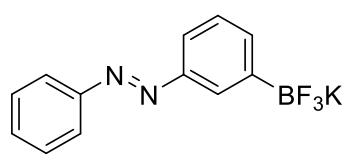

Prepared from (E)-1-Phenyl-2-(3-(4,4,5,5-tetramethyl-1,3,2-dioxaborolan-2yl)phenyl)diazene $(274 \mathrm{mg}, 1.17 \mathrm{mmol})$ by using potassium bifluoride (274 mg, $3.51 \mathrm{mmol}$ ) following general procedure GP3 (stirred at $\mathrm{rt}$ for $4 \mathrm{~h}$ ). Purification by recrystallisation from $\mathrm{Et}_{2} \mathrm{O} / n$-pentane afforded $\mathbf{1}$ as an orange solid (126 mg, $0.44 \mathrm{mmol}, 38 \%$ ).

${ }^{1}$ H NMR (400 MHz, DMSO-d $\left.\mathbf{d}_{\mathbf{6}}\right): \delta 7.90-7.85(\mathrm{~m}, 3 \mathrm{H}), 7.63-7.50(\mathrm{~m}, 5 \mathrm{H}), 7.35-7.30(\mathrm{~m}, 1 \mathrm{H})$; ${ }^{13} \mathbf{C}\left\{{ }^{1} \mathbf{H}\right\}$ NMR (101 MHz, DMSO- $\left.\boldsymbol{d}_{\mathbf{6}}\right): \delta 152.2,151.0,135.1,130.7,129.3,128.9,127.3,125.2,122.2$, 120.1; ${ }^{19}$ F NMR (377 MHz, DMSO- $\left.\boldsymbol{d}_{6}\right): \delta-139.5 ;{ }^{11}$ B NMR (128 MHz, DMSO- $\left.\boldsymbol{d}_{6}\right): \delta 3.2$ (bs); HRMS (ESI-): $m / z$ calculated for $\mathrm{C}_{12} \mathrm{H}_{9} \mathrm{BF}_{3} \mathrm{~N}_{2}^{-}\left(\left[\mathrm{M}-\mathrm{K}^{+}\right]^{-}\right)$249.0816, found 249.0814.

\section{(E)-1-phenyl-2-(4-(trifluoro-14-boraneyl)phenyl)diazene, potassium salt (2)}<smiles></smiles>

Prepared from (E)-1-Phenyl-2-(4-(4,4,5,5-tetramethyl-1,3,2-dioxaborolan-2yl)phenyl)diazene $(310 \mathrm{mg}, 1.37 \mathrm{mmol})$ by using potassium bifluoride (322 mg, $4.12 \mathrm{mmol}$ ) following general procedure GP3 (stirred at $\mathrm{rt}$ for $16 \mathrm{~h}$ ). solid (271 mg, $0.94 \mathrm{mmol}, 69 \%)$.

Purification by recrystallisation from DCM/ $n$-pentane afforded $\mathbf{2}$ as an orange

${ }^{1}$ H NMR (400 MHz, DMSO- $\left.\boldsymbol{d}_{\mathbf{6}}\right): \delta 7.88-7.83(\mathrm{~m}, 2 \mathrm{H}), 7.69-7.62(\mathrm{~m}, 2 \mathrm{H}), 7.61-7.50(\mathrm{~m}, 5 \mathrm{H})$; ${ }^{13} \mathbf{C}\left\{{ }^{1} \mathbf{H}\right\}$ NMR (101 MHz, DMSO- $\left.\boldsymbol{d}_{\mathbf{6}}\right): \delta$ 152.1, 150.3, 132.1, 130.7, 129.3, 129.3, 122.2, 120.8; ${ }^{19} \mathbf{F}$ NMR (377 MHz, DMSO-d $\boldsymbol{d}_{\mathbf{6}}$ ): $\delta-139.6 ;{ }^{11}$ B NMR (128 MHz, DMSO-d $\left.\boldsymbol{d}_{\mathbf{6}}\right): \delta 4.2$ (bs); HRMS (ESI-): $m / z$ calculated for $\mathrm{C}_{12} \mathrm{H}_{9} \mathrm{BF}_{3} \mathrm{~N}_{2}^{-}\left(\left[\mathrm{M}-\mathrm{K}^{+}\right]^{-}\right)$249.0816, found 249.0814 . 


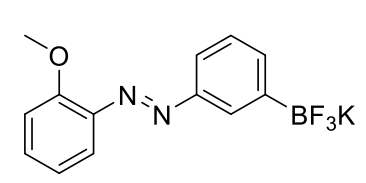

Prepared from (E)-1-(2-methoxyphenyl)-2-(3-(4,4,5,5-tetramethyl-1,3,2dioxaborolan-2-yl)phenyl)diazene $(170.2 \mathrm{mg}, 0.5 \mathrm{mmol})$ by using potassium bifluoride (118 mg, $1.5 \mathrm{mmol}$ ) following general procedure GP3 (stirred at $\mathrm{rt}$ for $4 \mathrm{~h}$ ). Purification by recrystallisation from $\mathrm{DCM} / \mathrm{Et}_{2} \mathrm{O}$ afforded $\mathbf{4}$ as an orange solid (44.1 mg, $0.14 \mathrm{mmol}, 28 \%$ ). The final compound contains an unknown impurity or isomer that could not be removed ( $30 \%$ based on ${ }^{1} \mathrm{H}$ NMR analysis).

${ }^{1}$ H NMR (400 MHz, DMSO-d $\left.\boldsymbol{d}_{\mathbf{6}}\right): \delta 7.87(\mathrm{~s}, 1 \mathrm{H}), 7.59-7.43(\mathrm{~m}, 4 \mathrm{H}), 7.35-7.21(\mathrm{~m}, 2 \mathrm{H}), 7.07-6.99$ (m, 1H), $3.96(\mathrm{~s}, 3 \mathrm{H}) ;{ }^{13} \mathbf{C}\left\{{ }^{1} \mathbf{H}\right\}$ NMR (101 MHz, DMSO-d $\left.\mathbf{d}_{\mathbf{6}}\right): \delta 156.4,151.5,141.7,132.1,131.9,127.6$, 127.1, $124.7,120.5,116.2,113.3,112.0,55.9 ;{ }^{19}$ F NMR (377 MHz, DMSO-d $\left.\boldsymbol{d}_{6}\right): \delta-139.5 ;{ }^{11} \mathbf{B}$ NMR (128 MHz, DMSO-d $\left.\boldsymbol{d}_{6}\right): \delta 3.1$ (bs); HRMS (ESI-): $\mathrm{m} / z$ calculated for $\mathrm{C}_{13} \mathrm{H}_{11} \mathrm{BF}_{3} \mathrm{~N}_{2} \mathrm{O}^{-}\left(\left[\mathrm{M}^{-} \mathrm{K}^{+}\right]^{-}\right)$ 279.0924, found 279.0920.

(E)-1-(2-iodophenyl)-2-(3-(trifluoro-14-boraneyl)phenyl)diazene, potassium salt (5)<smiles>Cc1cccc(N=Nc2ccccc2I)c1</smiles>

Prepared from (E)-1-(2-Iodophenyl)-2-(3-(4,4,5,5-tetramethyl-1,3,2dioxaborolan-2-yl)phenyl)diazene $(240 \mathrm{mg}, 0.55 \mathrm{mmol})$ by using potassium bifluoride (130 mg, $1.65 \mathrm{mmol}$ ) following general procedure GP3 (stirred at $\mathrm{rt}$ for $5 \mathrm{~h}$ ). Purification by recrystallisation from $\mathrm{Et}_{2} \mathrm{O} / n$-pentane afforded 5 as an orange solid ( $87 \mathrm{mg}, 0.25 \mathrm{mmol}, 45 \%)$.

${ }^{1}$ H NMR (400 MHz, DMSO-d $\left.\mathbf{6}_{\mathbf{6}}\right): \delta 8.11-8.06(\mathrm{~m}, 1 \mathrm{H}), 7.94(\mathrm{~s}, 1 \mathrm{H}), 7.65$ (ddd, $J=7.8,2.2,1.3 \mathrm{~Hz}$, $1 \mathrm{H}), 7.58-7.51(\mathrm{~m}, 3 \mathrm{H}), 7.38-7.32(\mathrm{~m}, 1 \mathrm{H}), 7.27(\mathrm{ddd}, J=7.8,6.4,2.4 \mathrm{~Hz}, 1 \mathrm{H}) ;{ }^{13} \mathbf{C}\left\{{ }^{1} \mathbf{H}\right\} \mathbf{~ N M R}$ (101 MHz, DMSO- $\left.\boldsymbol{d}_{\mathbf{6}}\right): \delta 151.2,150.9,139.6,135.7,132.3,129.3,127.4,127.0,126.3,120.2,117.2$, 101.7; ${ }^{19}$ F NMR (377 MHz, DMSO-d $)$ ) $\delta-139.6 ;{ }^{11}$ B NMR (128 MHz, DMSO- $\boldsymbol{d}_{\mathbf{6}}$ ): $\delta 3.3$ (bs); HRMS (ESI-): $m / z$ calculated for $\mathrm{C}_{12} \mathrm{H}_{8} \mathrm{BF}_{3} \mathrm{IN}_{2}^{-}\left(\left[\mathrm{M}-\mathrm{K}^{+}\right]^{-}\right) 374.9785$, found 374.9778 .

(E)-1-(2,6-difluorophenyl)-2-(3-(trifluoro-14-boraneyl)phenyl)diazene, potassium salt (6)<smiles>Fc1cccc(F)c1N=Nc1cccc(Br)c1</smiles>

Prepared from (E)-1-(2,6-difluorophenyl)-2-(3-(4,4,5,5-tetramethyl-1,3,2dioxaborolan-2-yl)phenyl)diazene $(390 \mathrm{mg}, 1.13 \mathrm{mmol})$ by using potassium bifluoride (266 mg, $3.40 \mathrm{mmol}$ ) following general procedure GP3 (stirred at $\mathrm{rt}$ for $7 \mathrm{~h}$ ). Purification by recrystallisation from $\mathrm{Et}_{2} \mathrm{O} / n$-pentane afforded 6 as an orange solid $160 \mathrm{mg}, 0.49 \mathrm{mmol}, 44 \%)$.

${ }^{1}$ H NMR (400 MHz, DMSO-d $\left.\boldsymbol{d}_{6}\right): \delta 7.85(\mathrm{~s}, 1 \mathrm{H}), 7.60(\mathrm{dd}, J=7.6,1.9 \mathrm{~Hz}, 2 \mathrm{H}), 7.56-7.47(\mathrm{~m}, 1 \mathrm{H})$, 7.39 - $7.28(\mathrm{~m}, 3 \mathrm{H}) ;{ }^{13} \mathbf{C}\left\{{ }^{1} \mathbf{H}\right\}$ NMR (101 MHz, DMSO-d $\left.\boldsymbol{d}_{\mathbf{6}}\right): \delta 156.0,153.4,151.7,136.2,130.7,130.6$, 127.5, 125.0, 120.3, 113.0; ${ }^{19} \mathbf{F}$ NMR (377 MHz, DMSO-d $\left.\mathbf{6}\right): \delta-123.3(\mathrm{~m}, 2 \mathrm{~F}),-139.7(\mathrm{~s}, 3 \mathrm{~F}) ;{ }^{11} \mathbf{B}$ NMR (128 MHz, DMSO-d $\left.\boldsymbol{d}_{6}\right): \delta 3.2$ (bs); HRMS (ESI-): $m / z$ calculated for $\mathrm{C}_{12} \mathrm{H}_{7} \mathrm{BF}_{4} \mathrm{~N}_{2}^{-}\left(\left[\mathrm{M}^{-} \mathrm{K}^{+}\right]^{-}\right)$ 285.0630 , found 285.0624 . 
(E)-1-(2-isopropylphenyl)-2-(3-(trifluoro-14-boraneyl)phenyl)diazene, potassium salt (7)

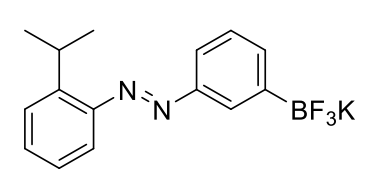

Prepared from (E)-1-(2-isopropylphenyl)-2-(3-(4,4,5,5-tetramethyl-1,3,2dioxaborolan-2-yl)phenyl)diazene (366 $\mathrm{mg}, 1.05 \mathrm{mmol})$ by using potassium bifluoride (245 mg, $3.15 \mathrm{mmol}$ ) following general procedure $\mathbf{G P 3}$ (stirred at rt for $16 \mathrm{~h}$ ). Purification by recrystallisation from $\mathrm{Et}_{2} \mathrm{O} / n$-pentane afforded 7 as a red-orange solid (158 $\mathrm{mg}, 0.48 \mathrm{mmol}, 46 \%$ ).

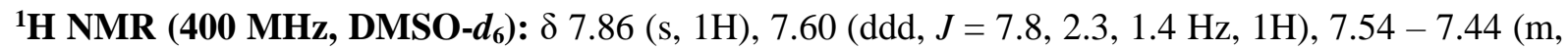
4H), $7.37-7.28(\mathrm{~m}, 2 \mathrm{H}), 4.04(\mathrm{p}, J=7.0 \mathrm{~Hz}, 1 \mathrm{H}), 1.31(\mathrm{~s}, 3 \mathrm{H}), 1.30(\mathrm{~s}, 3 \mathrm{H}) ;{ }^{13} \mathbf{C}\left\{{ }^{1} \mathbf{H}\right\}$ NMR (101 MHz, DMSO- $\left.\boldsymbol{d}_{\mathbf{6}}\right): \delta 151.5,149.2,146.7,134.9,130.9,127.3,126.5,126.4,125.4,125.3,120.1,115.0,27.2$, 23.7; ${ }^{19}$ F NMR (377 MHz, DMSO- $\left.\boldsymbol{d}_{\mathbf{6}}\right): \delta-139.5 ;{ }^{11} \mathrm{~B}$ NMR (128 MHz, DMSO- $\left.\boldsymbol{d}_{\mathbf{6}}\right): \delta 3.4$ (bs); HRMS (ESI-): $\mathrm{m} / z$ calculated for $\mathrm{C}_{15} \mathrm{H}_{15} \mathrm{BF}_{3} \mathrm{~N}_{2}^{-}\left(\left[\mathrm{M}^{-} \mathrm{K}^{+}\right]^{-}\right)$291.1288, found 291.1281.

\section{(E)-1-(o-tolyl)-2-(3-(trifluoro-14-boraneyl)phenyl)diazene, potassium salt (8)}<smiles>Cc1ccccc1N=Nc1cccc(Br)c1</smiles>

Prepared from (E)-1-(3-(4,4,5,5-tetramethyl-1,3,2-dioxaborolan-2-yl)phenyl)2-(o-tolyl)diazene $(411 \mathrm{mg}, 1.27 \mathrm{mmol})$ by using potassium bifluoride (299.1 mg, $3.83 \mathrm{mmol}$ ) following general procedure GP3 (stirred at rt for $6 \mathrm{~h}$ ). Purification by recrystallisation from $\mathrm{Et}_{2} \mathrm{O} / n$-pentane afforded $\mathbf{8}$ as an orange solid (36.0 mg, $0.12 \mathrm{mmol}, 10 \%)$.

${ }^{1}$ H NMR (400 MHz, DMSO-d $\left.\mathbf{6}_{6}\right): \delta 7.87(\mathrm{~s}, 1 \mathrm{H}), 7.60(\mathrm{ddd}, J=7.8,2.3,1.4 \mathrm{~Hz}, 1 \mathrm{H}), 7.56-7.50(\mathrm{~m}$, 2H), $7.43-7.38(\mathrm{~m}, 2 \mathrm{H}), 7.35$ - $7.28(\mathrm{~m}, 2 \mathrm{H}), 2.67$ (s, 3H); ${ }^{13} \mathbf{C}\left\{{ }^{1} \mathbf{H}\right\}$ NMR (101 MHz, DMSO-d $\left.\boldsymbol{d}_{\mathbf{6}}\right): \delta$ 151.4, 150.3, 136.9, 134.9, 131.3, 130.6, 127.2, 126.6, 125.5, 119.9, 115.1, 17.1; ${ }^{19}$ F NMR (377 MHz, DMSO-d $\left.\boldsymbol{d}_{\mathbf{6}}\right): \delta-139.5 ;{ }^{11} \mathrm{~B}$ NMR (128 MHz, DMSO- $\boldsymbol{d}_{\mathbf{6}}$ ): $\delta 3.3$ (bs); HRMS (ESI-): $\mathrm{m} / \mathrm{z}$ calculated for $\mathrm{C}_{13} \mathrm{H}_{11} \mathrm{BF}_{3} \mathrm{~N}_{2}^{-}\left(\left[\mathrm{M}-\mathrm{K}^{+}\right]^{-}\right)$263.0975, found 263.0968.

\section{(E)-1-(m-tolyl)-2-(3-(trifluoro-l4-boraneyl)phenyl)diazene, potassium salt (9)}

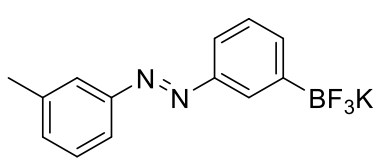

Prepared from (E)-1-(3-(4,4,5,5-tetramethyl-1,3,2-dioxaborolan-2yl)phenyl)-2-( $m$-tolyl)diazene $(574 \mathrm{mg}, 1.78 \mathrm{mmol})$ by using potassium bifluoride (417 mg, $5.35 \mathrm{mmol}$ ) following general procedure GP3 (stirred at $\mathrm{rt}$ for $4 \mathrm{~h}$ ). Purification by recrystallisation from $\mathrm{Et}_{2} \mathrm{O} / n$-pentane afforded 9 as an orange solid (142 $\mathrm{mg}, 0.47 \mathrm{mmol}, 26 \%)$.

${ }^{1}$ H NMR (400 MHz, DMSO-d $\left.\mathbf{6}_{6}\right): \delta 7.84(\mathrm{~s}, 1 \mathrm{H}), 7.78(\mathrm{~d}, J=8.3 \mathrm{~Hz}, 2 \mathrm{H}), 7.57$ (ddd, $J=7.9,2.3,1.3$ $\mathrm{Hz}, 1 \mathrm{H}), 7.50(\mathrm{dt}, J=7.2,1.2 \mathrm{~Hz}, 1 \mathrm{H}), 7.41-7.36(\mathrm{~m}, 2 \mathrm{H}), 7.34-7.28(\mathrm{~m}, 1 \mathrm{H}), 2.40(\mathrm{~s}, 3 \mathrm{H}) ;{ }^{13} \mathbf{C}\left\{{ }^{1} \mathbf{H}\right\}$ NMR (101 MHz, DMSO-d $\left.\boldsymbol{d}_{\mathbf{6}}\right): \delta$ 150.9, 150.3, 140.8, 136.3, 134.8, 129.8, 129.2, 127.2, 125.0, 122.2, 120.2, $120.0,21.0 ;{ }^{19}$ F NMR (377 MHz, DMSO- $\left.\boldsymbol{d}_{\mathbf{6}}\right): \delta-139.5 ;{ }^{11}$ B NMR (128 MHz, DMSO- $\left.\boldsymbol{d}_{\mathbf{6}}\right): \delta 2.8$ (bs); HRMS (ESI-): $\mathrm{m} / z$ calculated for $\mathrm{C}_{13} \mathrm{H}_{11} \mathrm{BF}_{3} \mathrm{~N}_{2}^{-}\left(\left[\mathrm{M}^{-} \mathrm{K}^{+}\right]^{-}\right)$263.0975, found 263.0969. 


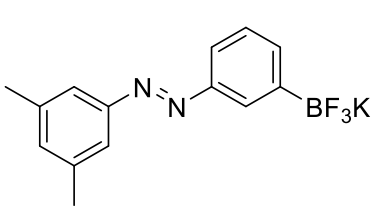

Prepared from (E)-1-(3,5-dimethylphenyl)-2-(3-(4,4,5,5-tetramethyl-1,3,2dioxaborolan-2-yl)phenyl)diazene $(200 \mathrm{mg}, 0.6 \mathrm{mmol})$ by using potassium bifluoride (140 mg, $1.80 \mathrm{mmol}$ ) following general procedure GP3 (stirred at $\mathrm{rt}$ for $16 \mathrm{~h}$ ). Purification by recrystallisation from DCM $/ n$-pentane afforded $\mathbf{1 0}$ as an orange solid $(110 \mathrm{mg}, 0.35 \mathrm{mmol}, 58 \%)$.

${ }^{1}$ H NMR (400 MHz, DMSO-d $\left.\mathbf{6}_{6}\right): \delta 7.84(\mathrm{~s}, 1 \mathrm{H}), 7.57(\mathrm{ddd}, J=7.7,2.2,1.3 \mathrm{~Hz}, 1 \mathrm{H}), 7.52-7.47(\mathrm{~m}$, $3 \mathrm{H}), 7.31$ (ddt, $J=8.0,7.2,0.7 \mathrm{~Hz}, 1 \mathrm{H}), 7.16(\mathrm{~s}, 1 \mathrm{H}), 2.38$ (s, 6H); ${ }^{13} \mathbf{C}\left\{{ }^{1} \mathbf{H}\right\}$ NMR (101 MHz, DMSO$\left.\boldsymbol{d}_{6}\right): \delta 152.4,151.0,138.5,137.9,134.9,132.1,127.2,125.0,120.1,117.1,20.8 ;{ }^{19} \mathbf{F}$ NMR (377 MHz, DMSO-d $\boldsymbol{d}_{\mathbf{6}}$ : $\delta-139.5 ;{ }^{11} \mathrm{~B}$ NMR (128 MHz, DMSO-d $\left.\boldsymbol{d}_{\mathbf{6}}\right): \delta 3.1$ (bs); HRMS (ESI-): $\mathrm{m} / \mathrm{z}$ calculated for $\mathrm{C}_{14} \mathrm{H}_{13} \mathrm{BF}_{3} \mathrm{~N}_{2}^{-}\left(\left[\mathrm{M}-\mathrm{K}^{+}\right]^{-}\right)$277.1131, found 277.1126.

\section{(E)-1-(3-((3-(trifluoro-14-boraneyl)phenyl)diazenyl)phenyl)ethan-1-one, potassium salt (11)}

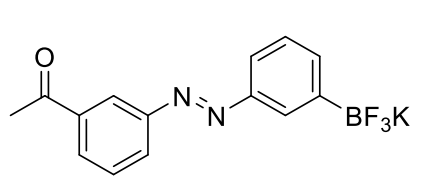

Prepared from (E)-1-(3-((3-(4,4,5,5-tetramethyl-1,3,2-dioxaborolan-2yl)phenyl)diazenyl)phenyl)ethan-1-one $(390 \mathrm{mg}, 1.11 \mathrm{mmol})$ by using potassium bifluoride $(261 \mathrm{mg}, 3.33 \mathrm{mmol})$ following general procedure GP3 (stirred at $\mathrm{rt}$ for $4 \mathrm{~h}$ ). Purification by recrystallisation from $\mathrm{MeCN} / n$ pentane afforded $\mathbf{1 1}$ as an orange solid (110.1 $\mathrm{mg}, 0.33 \mathrm{mmol}, 30 \%)$.

${ }^{1}$ H NMR (400 MHz, DMSO-d $\left.\boldsymbol{d}_{\mathbf{6}}\right): \delta 8.39(\mathrm{t}, J=1.8 \mathrm{~Hz}, 1 \mathrm{H}), 8.15-8.07(\mathrm{~m}, 2 \mathrm{H}), 7.92(\mathrm{~s}, 1 \mathrm{H}), 7.74(\mathrm{t}$, $J=7.8 \mathrm{~Hz}, 1 \mathrm{H}), 7.67(\mathrm{ddd}, J=7.9,2.3,1.3 \mathrm{~Hz}, 1 \mathrm{H}), 7.56(\mathrm{dt}, J=7.2,1.3 \mathrm{~Hz}, 1 \mathrm{H}), 7.38-7.32(\mathrm{~m}, 1 \mathrm{H})$, 2.70 (s, 3H); ${ }^{13} \mathbf{C}\left\{{ }^{1} \mathbf{H}\right\}$ NMR (101 MHz, DMSO-d $\left.\mathbf{d}_{\mathbf{6}}\right): \delta$ 197.6, 152.3, 150.9, 137.9, 135.5, 130.0, 129.9, 127.4, 126.3, 125.3, 125.2, 121.9, 120.5, 27.0; ${ }^{19} \mathbf{F}$ NMR (377 MHz, DMSO- $\left.d_{6}\right): \delta-139.6 ;{ }^{11} \mathbf{B}$ NMR (128 MHz, DMSO-d $\left.\boldsymbol{d}_{6}\right): \delta 2.9$ (bs); HRMS (ESI-): $\mathrm{m} / z$ calculated for $\mathrm{C}_{14} \mathrm{H}_{11} \mathrm{BF}_{3} \mathrm{~N}_{2} \mathrm{O}^{-}\left(\left[\mathrm{M}^{-} \mathrm{K}^{+}\right]^{-}\right)$ 291.0924, found 291.0917.

(E)-1-(4-butylphenyl)-2-(3-(trifluoro-14-boraneyl)phenyl)diazene, potassium salt (12)

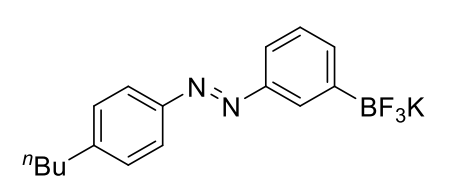

Prepared from (E)-1-(4-butylphenyl)-2-(3-(4,4,5,5-tetramethyl-1,3,2dioxaborolan-2-yl)phenyl)diazene $(397.5 \mathrm{mg}, 1.15 \mathrm{mmol})$ by using potassium bifluoride $(270 \mathrm{mg}, 3.45 \mathrm{mmol}$ ) following general procedure GP3 (stirred at rt for $16 \mathrm{~h}$ ). Purification by recrystallisation from $\mathrm{Et}_{2} \mathrm{O} / n$ pentane afforded 12 as an orange solid (79.2 $\mathrm{mg}, 0.23 \mathrm{mmol}, 20 \%)$.

${ }^{1}$ H NMR (400 MHz, DMSO-d $\left.d_{6}\right): \delta 7.86(\mathrm{~s}, 1 \mathrm{H}), 7.83-7.77(\mathrm{~m}, 2 \mathrm{H}), 7.58(\mathrm{ddd}, J=7.8,2.3,1.4 \mathrm{~Hz}$, $1 \mathrm{H}), 7.51(\mathrm{dt}, J=7.2,1.2 \mathrm{~Hz}, 1 \mathrm{H}), 7.42-7.37(\mathrm{~m}, 2 \mathrm{H}), 7.35-7.28(\mathrm{~m}, 1 \mathrm{H}), 2.67(\mathrm{t}, J=7.7 \mathrm{~Hz}, 2 \mathrm{H})$, $1.65-1.55(\mathrm{~m}, 2 \mathrm{H}), 1.40-1.28(\mathrm{~m}, 2 \mathrm{H}), 0.92(\mathrm{t}, J=7.3 \mathrm{~Hz}, 3 \mathrm{H}) ;{ }^{13} \mathbf{C}\left\{{ }^{1} \mathbf{H}\right\}$ NMR (101 MHz, DMSO$\left.\boldsymbol{d}_{6}\right): \delta 151.0,150.5,145.6,134.8,129.2,127.2,125.1,125.0,122.3,112.0,34.6,32.9,21.7,13.8 ;{ }^{19} \mathbf{F}$ NMR (377 MHz, DMSO-d $\left.\boldsymbol{d}_{6}\right): \delta-139.5 ;{ }^{11}$ B NMR (128 MHz, DMSO-d $\left.\boldsymbol{d}_{6}\right): \delta 3.4$ (bs); HRMS (ESI-): $m / z$ calculated for $\mathrm{C}_{16} \mathrm{H}_{17} \mathrm{BF}_{3} \mathrm{~N}_{2}^{-}\left(\left[\mathrm{M}-\mathrm{K}^{+}\right]^{-}\right) 305.1445$, found 305.1437 . 


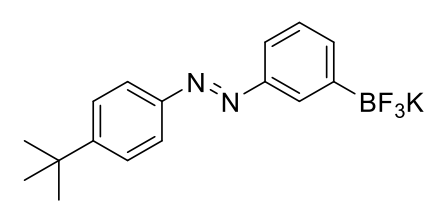

Prepared from (E)-1-(4-('butyl)phenyl)-2-(3-(4,4,5,5-tetramethyl-1,3,2dioxaborolan-2-yl)phenyl)diazene $(483 \mathrm{mg}, \quad 1.33 \mathrm{mmol})$ by using potassium bifluoride $(310 \mathrm{mg}, 3.98 \mathrm{mmol})$ following general procedure GP3 (stirred at $\mathrm{rt}$ for $16 \mathrm{~h}$ ). Purification by recrystallisation from $\mathrm{Et}_{2} \mathrm{O} / n$ pentane afforded 13 as an orange solid (216 mg, $0.62 \mathrm{mmol}, 47 \%)$.

${ }^{1}$ H NMR (400 MHz, DMSO-d $\left.\boldsymbol{d}_{6}\right): \delta 7.86(\mathrm{~s}, 1 \mathrm{H}), 7.83-7.79(\mathrm{~m}, 2 \mathrm{H}), 7.62-7.57(\mathrm{~m}, 3 \mathrm{H}), 7.50$ (dt, $J=$ 7.2, $1.3 \mathrm{~Hz}, 1 \mathrm{H}), 7.34-7.28(\mathrm{~m}, 1 \mathrm{H}), 1.34(\mathrm{~s}, 9 \mathrm{H}) ;{ }^{13} \mathbf{C}\left\{{ }^{1} \mathbf{H}\right\}$ NMR (101 MHz, DMSO-d $)_{6}: \delta 153.6$, 151.0, 150.2, 134.8, 127.2, 126.1, 125.5, 125.1, 122.0, 120.0, 34.7, 31.0; ${ }^{19}$ F NMR (377 MHz, DMSO$\left.\boldsymbol{d}_{\mathbf{6}}\right): \delta-139.6$; ${ }^{11} \mathbf{B}$ NMR (128 MHz, DMSO-d $\left.{ }_{6}\right): \delta 3.3$ (bs); HRMS (ESI-): $\mathrm{m} / \mathrm{z}$ calculated for $\mathrm{C}_{16} \mathrm{H}_{17} \mathrm{BF}_{3} \mathrm{~N}_{2}^{-}\left(\left[\mathrm{M}-\mathrm{K}^{+}\right]^{-}\right)$305.1445, found 305.1439.

(E)-4-((3-(trifluoro-14-boraneyl)phenyl)diazenyl)benzoic acid, potassium salt (14)<smiles>O=C(O)c1ccc(N=Nc2cccc(Br)c2)cc1</smiles>

Prepared from (E)-4-((3-(4,4,5,5-tetramethyl-1,3,2-dioxaborolan-2yl)phenyl)diazenyl)benzoic acid ( $70 \mathrm{mg}, 0.31 \mathrm{mmol})$ by using potassium bifluoride (73 mg, $0.93 \mathrm{mmol}$ ) following general procedure GP3 (stirred at $\mathrm{rt}$ for $16 \mathrm{~h}$ ). Purification by recrystallisation from $\mathrm{Et}_{2} \mathrm{O} / n$-pentane afforded 14 as an orange solid (52.3 $\mathrm{mg}, 0.15 \mathrm{mmol}, 48 \%)$.

${ }^{1}$ H NMR (400 MHz, Methanol- $\left.\boldsymbol{d}_{4}\right): \delta 8.15-8.08(\mathrm{~m}, 3 \mathrm{H}), 7.90-7.86(\mathrm{~m}, 2 \mathrm{H}), 7.73-7.66(\mathrm{~m}, 2 \mathrm{H})$, $7.40-7.34(\mathrm{~m}, 1 \mathrm{H}) ;{ }^{13} \mathbf{C}\left\{{ }^{1} \mathbf{H}\right\}$ NMR (101 MHz, Methanol- $\left.\boldsymbol{d}_{4}\right): \delta$ 172.9, 155.8, 153.3, 138.6, 136.4, 131.3, 128.6, 127.7, 127.6, 123.0, 121.3; ${ }^{19} \mathbf{F}$ NMR (377 MHz, Methanol- $\left.d_{4}\right): \delta-154.3 ;{ }^{11}$ B NMR (128 MHz, Methanol- $\left.d_{4}\right): \delta 3.6$ (bs); HRMS (ESI-): $m / z$ calculated for $\mathrm{C}_{13} \mathrm{H}_{9} \mathrm{BF}_{3} \mathrm{~N}_{2} \mathrm{O}_{2}^{-}\left(\left[\mathrm{M}-\mathrm{K}^{+}\right]^{2-}\right)$ 146.0322, found 146.0321 .

(E)-1-(4-((3-(trifluoro-I4-boraneyl)phenyl)diazenyl)phenyl)ethan-1-one, potassium salt (16)

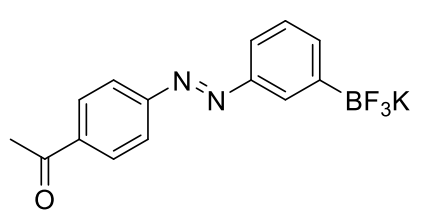

Prepared from (E)-1-(4-((3-(4,4,5,5-tetramethyl-1,3,2-dioxaborolan-2yl)phenyl)diazenyl)phenyl)ethan-1-one $(419 \mathrm{mg}, 1.20 \mathrm{mmol})$ by using potassium bifluoride $(281 \mathrm{mg}, 3.60 \mathrm{mmol})$ following general procedure GP3 (stirred at $\mathrm{rt}$ for $4 \mathrm{~h}$ ). Purification by recrystallisation from $\mathrm{MeOH} / \mathrm{Et}_{2} \mathrm{O}$ afforded 16 as orange solid (207.4 mg, $\left.0.63 \mathrm{mmol}, 52 \%\right)$.

${ }^{1}$ H NMR (400 MHz, DMSO-d $\left.\mathbf{6}_{6}\right): \delta 8.19-8.11(\mathrm{~m}, 2 \mathrm{H}), 7.99-7.94(\mathrm{~m}, 2 \mathrm{H}), 7.92(\mathrm{~s}, 1 \mathrm{H}), 7.66$ (ddd, $J=7.8,2.2,1.3 \mathrm{~Hz}, 1 \mathrm{H}), 7.58-7.54(\mathrm{~m}, 1 \mathrm{H}), 7.35(\mathrm{ddt}, J=7.9,7.2,0.7 \mathrm{~Hz}, 1 \mathrm{H}), 2.65(\mathrm{~s}, 3 \mathrm{H}) ;{ }^{13} \mathbf{C}\left\{{ }^{1} \mathbf{H}\right\}$ NMR (101 MHz, DMSO-d $\left.\mathbf{d}_{\mathbf{6}}\right): \delta$ 197.5, 154.6, 151.1, 137.8, 135.9, 129.6, 127.4, 125.5, 125.4, 122.6, 120.6, 27.0; ${ }^{19}$ F NMR (377 MHz, DMSO-d $\left.\boldsymbol{d}_{6}\right): \delta-139.6 ;{ }^{11} \mathbf{B}$ NMR (128 MHz, DMSO-d $\left.\boldsymbol{d}_{6}\right): \delta 3.2(\mathrm{bs})$; HRMS (ESI-): $m / z$ calculated for $\mathrm{C}_{14} \mathrm{H}_{11} \mathrm{BF}_{3} \mathrm{~N}_{2} \mathrm{O}^{-}\left(\left[\mathrm{M}^{-} \mathrm{K}^{+}\right]^{-}\right)$291.0924, found 291.0918. 


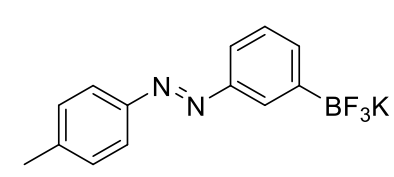

Prepared from (E)-1-(3-(4,4,5,5-tetramethyl-1,3,2-dioxaborolan-2yl)phenyl)-2-(o-tolyl)diazene (230 mg, $0.71 \mathrm{mmol})$ by using potassium bifluoride (167 mg, $2.14 \mathrm{mmol}$ ) following general procedure GP3 (stirred at $\mathrm{rt}$ for $4 \mathrm{~h}$ ). Purification by recrystallisation from $\mathrm{Et}_{2} \mathrm{O} / n$-pentane afforded 17 as an orange solid (77 $\mathrm{mg}, 0.25 \mathrm{mmol}, 36 \%)$.

${ }^{1}$ H NMR (400 MHz, DMSO-d $\left.\mathbf{6}_{6}\right): \delta 7.87$ (s, 1H), $7.70-7.64(\mathrm{~m}, 2 \mathrm{H}), 7.60$ (ddd, $J=7.8,2.2,1.3 \mathrm{~Hz}$, $1 \mathrm{H}), 7.52(\mathrm{dt}, J=7.2,1.2 \mathrm{~Hz}, 1 \mathrm{H}), 7.46(\mathrm{td}, J=7.4,1.0 \mathrm{~Hz}, 1 \mathrm{H}), 7.37-7.29(\mathrm{~m}, 2 \mathrm{H}), 2.43(\mathrm{~s}, 3 \mathrm{H})$; ${ }^{13} \mathbf{C}\left\{{ }^{1} \mathbf{H}\right\}$ NMR (101 MHz, DMSO-d $\left.\mathbf{6}\right): \delta$ 152.3, 151.0, 138.8, 135.0, 131.4, 129.1, 127.2, 125.1, 122.3, 120.1, 20.9; ${ }^{19}$ F NMR (377 MHz, DMSO- $\left.\boldsymbol{d}_{6}\right): \delta-139.5 ;{ }^{11}$ B NMR (128 MHz, DMSO- $\left.\boldsymbol{d}_{6}\right): \delta 3.2$ (bs); HRMS (ESI-): $\mathrm{m} / z$ calculated for $\mathrm{C}_{13} \mathrm{H}_{11} \mathrm{BF}_{3} \mathrm{~N}_{2}^{-}\left(\left[\mathrm{M}-\mathrm{K}^{+}\right]^{-}\right)$263.0975, found 263.0967.

\section{(E)-1-(2-methyl-3-(trifluoro-14-boraneyl)phenyl)-2-phenyldiazene, potassium salt (18)}

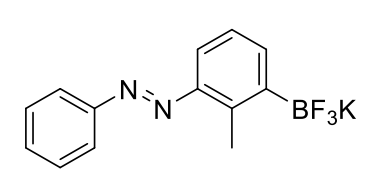

Prepared from (E)-1-(2-methyl-3-(4,4,5,5-tetramethyl-1,3,2-dioxaborolan-2yl)phenyl)-2-phenyldiazene $(310.2 \mathrm{mg}, 0.96 \mathrm{mmol})$ by using potassium bifluoride (225 mg, $2.88 \mathrm{mmol}$ ) following general procedure GP3 (stirred at $\mathrm{rt}$ for $16 \mathrm{~h}$ ). Purification by recrystallisation from DCM/n-pentane afforded 18 as an orange solid (138.9 mg, $0.46 \mathrm{mmol}, 48 \%$ ).

${ }^{1}$ H NMR (400 MHz, DMSO-d d $_{\text {): }} \delta 7.87$ - $7.81(\mathrm{~m}, 2 \mathrm{H}), 7.61-7.47(\mathrm{~m}, 4 \mathrm{H}), 7.31$ (dd, $J=8.0,1.5 \mathrm{~Hz}$, 1H), $7.07-7.01(\mathrm{~m}, 1 \mathrm{H}), 2.72(\mathrm{~s}, 3 \mathrm{H}) ;{ }^{13} \mathbf{C}\left\{{ }^{1} \mathbf{H}\right\}$ NMR (101 MHz, DMSO-d $\left.\mathbf{6}\right): \delta$ 152.7, 149.7, 141.6, 135.3, 130.4, 129.4, 128.7, 128.6, 124.1, 122.4, 122.3, 112.2, 15.6; ${ }^{19}$ F NMR (377 MHz, DMSO-d $\left.\boldsymbol{d}_{6}\right): \delta$ -137.1; ${ }^{11} \mathbf{B}$ NMR (128 MHz, DMSO-d $\boldsymbol{d}_{6}$ ): $\delta 3.4$ (bs); HRMS (ESI-): $m / z$ calculated for $\mathrm{C}_{13} \mathrm{H}_{11} \mathrm{BF}_{3} \mathrm{~N}_{2}{ }^{-}$ $\left(\left[\mathrm{M}-\mathrm{K}^{+}\right]^{-}\right)$263.0975, found 263.0969.

(E)-1-(4-methyl-3-(trifluoro-14-boraneyl)phenyl)-2-phenyldiazene, potassium salt (20/PI)

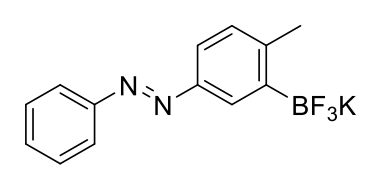

Prepared from (E)-1-(4-methyl-3-(4,4,5,5-tetramethyl-1,3,2-dioxaborolan-2yl)phenyl)-2-phenyldiazene (161 $\mathrm{mg}, 0.50 \mathrm{mmol}$ ) by using potassium bifluoride (118 mg, $1.50 \mathrm{mmol}$ ) following general procedure GP3 (stirred at $\mathrm{rt}$ for $4 \mathrm{~h}$ ). Purification by recrystallisation from $\mathrm{Et}_{2} \mathrm{O} / n$-pentane afforded 20 as an orange solid (132 mg, $0.44 \mathrm{mmol}, 87 \%)$.

${ }^{1}$ H NMR (400 MHz, DMSO-d $\left.\boldsymbol{d}_{\mathbf{6}}\right): \delta 7.91(\mathrm{~d}, J=2.2 \mathrm{~Hz}, 1 \mathrm{H}), 7.86-7.82(\mathrm{~m}, 2 \mathrm{H}), 7.59-7.53(\mathrm{~m}, 2 \mathrm{H})$, $7.52-7.48(\mathrm{~m}, 2 \mathrm{H}), 7.10(\mathrm{~d}, J=7.9 \mathrm{~Hz}, 1 \mathrm{H}), 2.40(\mathrm{~s}, 3 \mathrm{H}) ;{ }^{13} \mathbf{C}\left\{{ }^{1} \mathrm{H}\right\}$ NMR (101 MHz, DMSO-d $\left.\boldsymbol{d}_{6}\right): \delta$ 152.3, 149.1, 145.9, 130.4, 129.3, 128.9, 126.1, 122.1, 120.1, 119.4, 21.7; ${ }^{19}$ F NMR (377 MHz, DMSO$\left.\boldsymbol{d}_{\mathbf{6}}\right): \delta-138.1 ;{ }^{11} \mathrm{~B}$ NMR (128 MHz, DMSO- $\left.\boldsymbol{d}_{\mathbf{6}}\right): \delta 3.0$ (bs); HRMS (ESI-): $\mathrm{m} / \mathrm{z}$ calculated for $\mathrm{C}_{13} \mathrm{H}_{11} \mathrm{BF}_{3} \mathrm{~N}_{2}^{-}\left(\left[\mathrm{M}-\mathrm{K}^{+}\right]^{-}\right)$263.0975, found 263.0969. 


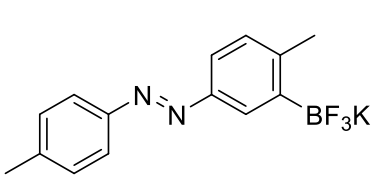

Prepared from (E)-1-(4-methyl-3-(4,4,5,5-tetramethyl-1,3,2-dioxaborolan-2yl)phenyl)-2-(p-tolyl)diazene (168 $\mathrm{mg}, 0.5 \mathrm{mmol})$ by using potassium bifluoride (118 $\mathrm{mg}, 1.5 \mathrm{mmol}$ ) following general procedure $\mathbf{G P 3}$ (stirred at $\mathrm{rt}$ for $6 \mathrm{~h}$ ). Purification by recrystallisation from $\mathrm{Et}_{2} \mathrm{O}$ afforded 21 as orange solid (84.3 mg, $0.27 \mathrm{mmol}, 54 \%)$.

${ }^{1}$ H NMR (400 MHz, DMSO-d $\left.\mathbf{6}\right): \delta 7.90(\mathrm{~d}, J=2.3 \mathrm{~Hz}, 1 \mathrm{H}), 7.75(\mathrm{~d}, J=8.3 \mathrm{~Hz}, 2 \mathrm{H}), 7.49$ (dd, $J=7.9$, $2.3 \mathrm{~Hz}, 1 \mathrm{H}), 7.38-7.34(\mathrm{~m}, 2 \mathrm{H}), 7.10(\mathrm{~d}, J=8.0 \mathrm{~Hz}, 1 \mathrm{H}), 2.40(\mathrm{~s}, 3 \mathrm{H}), 2.39(\mathrm{~s}, 3 \mathrm{H}) ;{ }^{13} \mathbf{C}\left\{{ }^{1} \mathbf{H}\right\} \mathbf{~ N M R}$ (101 MHz, DMSO- $\left.\boldsymbol{d}_{6}\right): \delta 150.4,149.1,145.5,140.3,129.8,129.2,129.1,126.0,122.1,119.9,21.7$, 20.9; ${ }^{19}$ F NMR (377 MHz, DMSO- $\left.\boldsymbol{d}_{\mathbf{6}}\right): \delta-138.1 ;{ }^{11}$ B NMR (128 MHz, DMSO-d $\left.\boldsymbol{d}_{\mathbf{6}}\right): \delta 3.4$ (bs); MS (ESI-): $\mathrm{m} / z$ calculated for $\mathrm{C}_{14} \mathrm{H}_{13} \mathrm{BF}_{3} \mathrm{~N}_{2}^{-}\left(\left[\mathrm{M}-\mathrm{K}^{+}\right]^{-}\right)$277.1, found 277.1. 


\subsection{Synthesis of Potassium Trifluoroborate-Based PI 3}

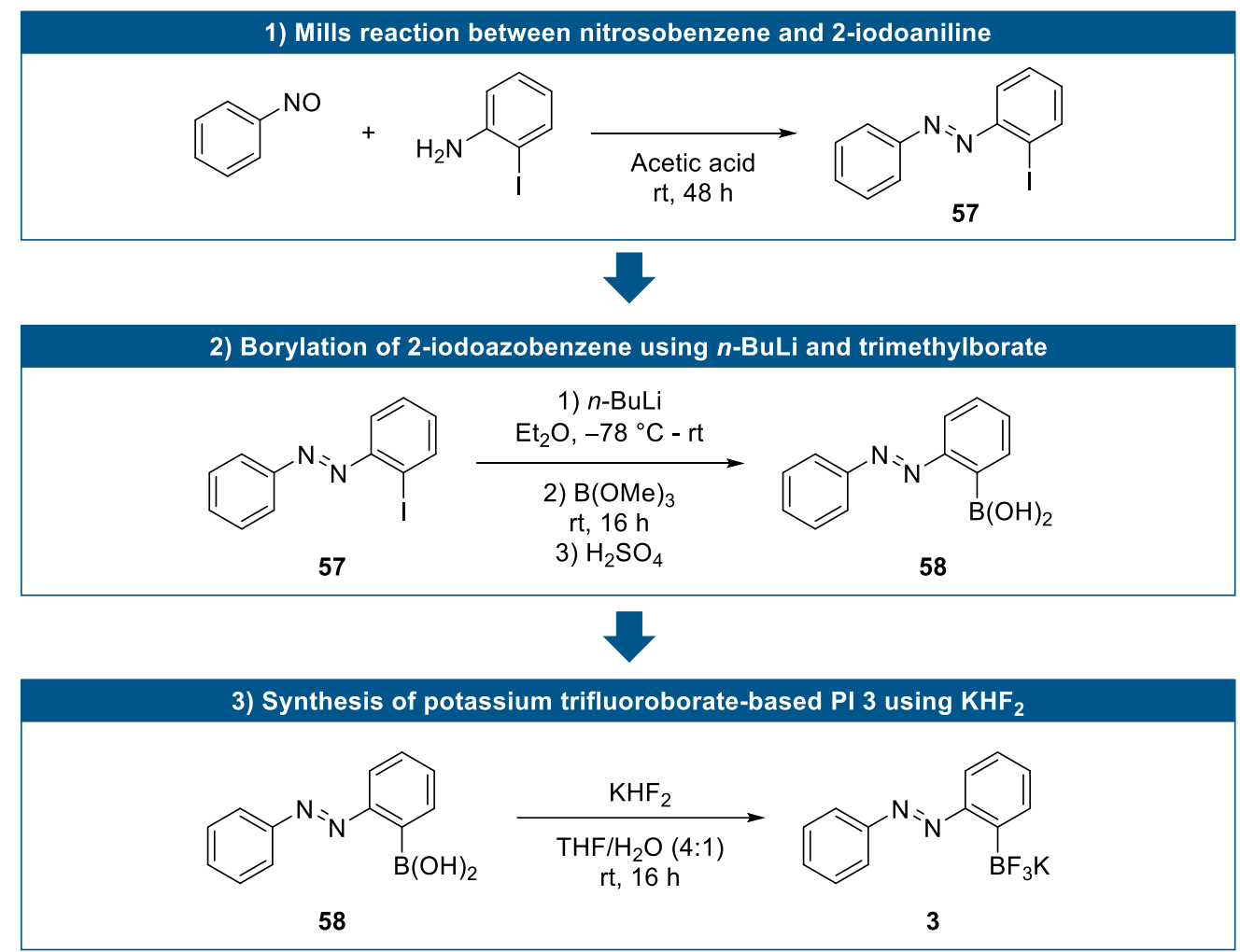

Figure S5. 3-Step synthetic procedure for the synthesis of potassium trifluoroborate-based PI 3.

(E)-1-Phenyl-2-(2-(trifluoro-14-boraneyl)phenyl)diazene (potassium salt) $\mathbf{3}$ was synthesized in three steps as outlined in Fig. S5. In the first step, 2-iodoazobenzene $\mathbf{5 7}$ was obtained via a Mills reaction between nitrosobenzene and 2-iodoaniline. Azobenzene 57 was then reacted in the second step to azobenzene boronic acid $\mathbf{5 8}$ using $n$-BuLi and trimethylborate (metal-halogen exchange). Finally, the boronic acid $\mathbf{5 8}$ was converted to the final potassium trifluoroborate-based PI $\mathbf{3}$ by the addition of potassium bifluoride.

\subsubsection{Mills Reaction to Obtain 2-Iodoazobenzene 57}

(E)-1-(2-iodophenyl)-2-phenyldiazene (57)<smiles>Ic1ccccc1N=Nc1ccccc1</smiles>

Prepared from nitrosobenzene $(214 \mathrm{mg}, 2.0 \mathrm{mmol}, 1.0$ equiv) and 2-iodoaniline (438 mg, $2.0 \mathrm{mmol}, 1.0$ equiv) following general procedure GP2 (stirred at $\mathrm{rt}$ for $48 \mathrm{~h}$ ). Purification by column chromatography over silica gel (eluent $=n$-pentane/DCM 1/1) afforded $\mathbf{5 7}$ as a red-orange oil (281 $\mathrm{mg}, 0.91 \mathrm{mmol}, 46 \%)$.

${ }^{1}$ H NMR (400 MHz, $\left.\mathbf{C D C l}_{3}\right): \delta 8.06-7.97(\mathrm{~m}, 3 \mathrm{H}), 7.64(\mathrm{dd}, J=8.0,1.6 \mathrm{~Hz}, 1 \mathrm{H}), 7.58-7.47(\mathrm{~m}$, $3 \mathrm{H}), 7.43(\mathrm{ddd}, J=8.0,7.2,1.3 \mathrm{~Hz}, 1 \mathrm{H}), 7.17(\mathrm{ddd}, J=7.9,7.2,1.6 \mathrm{~Hz}, 1 \mathrm{H}) ;{ }^{13} \mathbf{C}\left\{{ }^{1} \mathbf{H}\right\}$ NMR $(\mathbf{1 0 1} \mathbf{M H z}$, $\left.\mathbf{C D C l}_{3}\right): \delta 152.3,151.3,139.9,132.2,131.6,129.2,128.9,123.6,117.4,102.5 ; \mathbf{R}_{\mathbf{f}}(\mathbf{D C M}): 0.68$ 


\subsubsection{Synthesis of Azobenzene Boronic Acid 58}

\section{(E)-(2-(phenyldiazenyl)phenyl)boronic acid (58)}<smiles>Oc1ccccc1N=Nc1ccccc1</smiles>

(E)-(2-(phenyldiazenyl)phenyl)boronic acid $\mathbf{5 8}$ was synthesized following a literature report from Kano et al. ${ }^{4}$ Under an argon atmosphere, $n$-BuLi (1.60 M in hexane, $630 \mu \mathrm{L}, 1.01 \mathrm{mmol}, 1.1$ equiv) was added dropwise to a stirred $\mathrm{Et}_{2} \mathrm{O}$ solution $(10 \mathrm{~mL})$ of $(E)-1$-(2-iodophenyl)-2-phenyldiazene $\mathbf{5 7}(281 \mathrm{mg}$, $0.92 \mathrm{mmol}, 1.0$ equiv) at $-78{ }^{\circ} \mathrm{C}$. After the reaction solution was stirred for $30 \mathrm{~min}$ at $-78{ }^{\circ} \mathrm{C}$, an $\mathrm{Et}_{2} \mathrm{O}$ solution $(5 \mathrm{~mL})$ of trimethyl borate $(113 \mu \mathrm{L}, 1.01 \mathrm{mmol}, 1.1$ equiv) was added dropwise. The reaction temperature was gradually raised up to $\mathrm{rt}$ and the reaction mixture was stirred at room temperature for $12 \mathrm{~h}$. The yellow solution was quenched with water $(20 \mathrm{~mL})$ at $0{ }^{\circ} \mathrm{C}$ and $2 \mathrm{M}$ sulfuric acid $(2.5 \mathrm{~mL}) \mathrm{was}$ added. The organic layer was separated and the aqueous layer was extracted with $\mathrm{Et}_{2} \mathrm{O}(3 \times 30 \mathrm{~mL})$. The combined organic layers were treated with aq. $1 \mathrm{M} \mathrm{NaOH}(10 \mathrm{~mL})$, washed with $\mathrm{Et}_{2} \mathrm{O}(3 \times 50 \mathrm{~mL})$ and neutralized with $2 \mathrm{M}$ sulfuric acid. The precipitated yellow solid was dissolved in $\mathrm{Et}_{2} \mathrm{O}(30 \mathrm{~mL})$, the organic layer was separated, dried over anhydrous $\mathrm{MgSO}_{4}$, filtered and concentrated in vacuo. The residue was recrystallized from benzene to give boronic acid $\mathbf{5 8}$ as brown solid (93.5 $\mathrm{mg}, 0.41 \mathrm{mmol}$, $45 \%)$.

${ }^{1} \mathbf{H}$ NMR (400 MHz, CDCl$): ~ \delta 8.14-7.79(\mathrm{~m}, 4 \mathrm{H}), 7.61-7.33(\mathrm{~m}, 5 \mathrm{H}) ;{ }^{13} \mathbf{C}\left\{{ }^{1} \mathbf{H}\right\}$ NMR (101 MHz, $\left.\mathbf{C D C l}_{3}\right): \delta 156.8,152.2,136.3,131.8,131.5,129.5,129.3,123.0,122.8,115.5 ;{ }^{11} \mathbf{B}$ NMR (128 MHz, $\left.\mathbf{C D C l}_{3}\right): \delta 30.1$ (bs).

\subsubsection{Synthesis of Potassium Trifluoride-Based PI 3}

(E)-1-phenyl-2-(2-(trifluoro-14-boraneyl)phenyl)diazene, potassium salt (3)

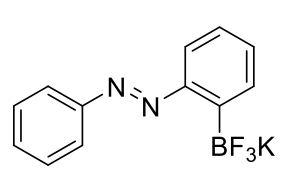

Azobenzene trifluoroborate 3 was synthesized following general procedure GP3. In a polyethylene round-bottom flask, azobenzene boronic acid $\mathbf{5 8}(88.6 \mathrm{mg}$, $0.39 \mathrm{mmol}, 1.0$ equiv) was dissolved in a $4: 1$ mixture of $\mathrm{THF}$ and water $(10 \mathrm{~mL})$ and potassium bifluoride ( $91.8 \mathrm{mg}, 1.17 \mathrm{mmol}, 3.0$ equiv) was added. After stirring at room temperature for $16 \mathrm{~h}$ (TLC indicated full consumption of starting material), $\mathrm{NaHCO}_{3}(49.1 \mathrm{mg}$, $0.56 \mathrm{mmol}, 1.5$ equiv) was added and the mixture was stirred for $10 \mathrm{~min}$. The volatiles were removed, the resulting oil was extracted with acetone $(20 \mathrm{~mL})$ and the extract was filtered. The solution was concentrated in vacuo. Pure potassium trifluoroborate PI 3 was obtained by recrystallisation from $\mathrm{Et}_{2} \mathrm{O} / \mathrm{n}$-pentane as orange solid $(31.6 \mathrm{mg}, 0.11 \mathrm{mmol}, 28 \%)$. The product contains unknown impurities which could not be separated ( $15 \%$ based on ${ }^{1} \mathrm{H}$ NMR analysis).

${ }^{1}$ H NMR (400 MHz, Methanol-d $\left.\mathbf{d}_{4}\right): \delta 8.15-8.08(\mathrm{~m}, 1 \mathrm{H}), 7.97-7.94(\mathrm{~m}, 1 \mathrm{H}), 7.80(\mathrm{dd}, J=7.3,1.7$ $\mathrm{Hz}, 1 \mathrm{H}), 7.64-7.61(\mathrm{~m}, 2 \mathrm{H}), 7.53-7.45(\mathrm{~m}, 3 \mathrm{H}), 7.36-7.29(\mathrm{~m}, 1 \mathrm{H}) ;{ }^{13} \mathbf{C}\left\{{ }^{1} \mathbf{H}\right\}$ NMR (101 MHz, Methanol- $\left.\boldsymbol{d}_{\mathbf{4}}\right): \delta 157.8,154.7,134.5,131.1,130.7,130.2,129.9,129.4,128.0,123.9 ;{ }^{19} \mathbf{F}$ NMR (377 MHz, Methanol- $\left.d_{4}\right): \delta-138.4 ;{ }^{11}$ B NMR (128 MHz, Methanol- $\left.d_{4}\right): \delta 4.1$ (bs); HRMS (ESI-): $m / z$ calculated for $\mathrm{C}_{12} \mathrm{H}_{9} \mathrm{BF}_{3} \mathrm{~N}_{2}^{-}\left(\left[\mathrm{M}-\mathrm{K}^{+}\right]^{-}\right)$249.0818, found 249.0815 . 


\subsection{Synthesis and Characterization Data of PI 15}

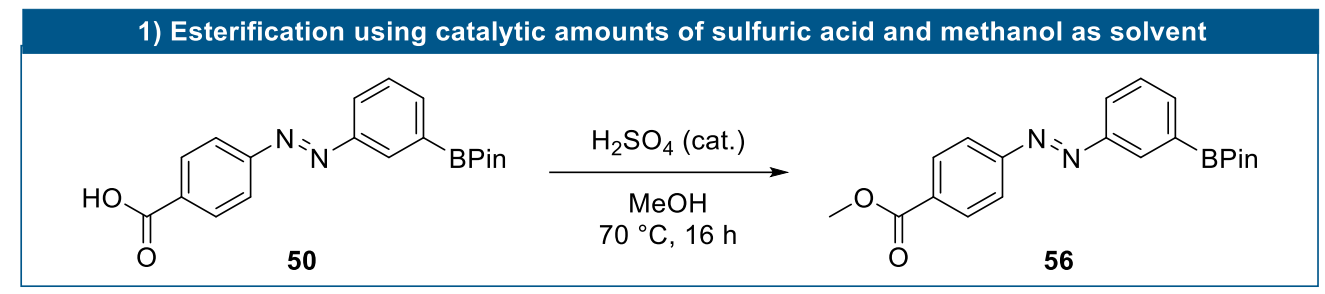

2) Synthesis of potassium trifluoroborate-based PI 15 using potassium bifluoride

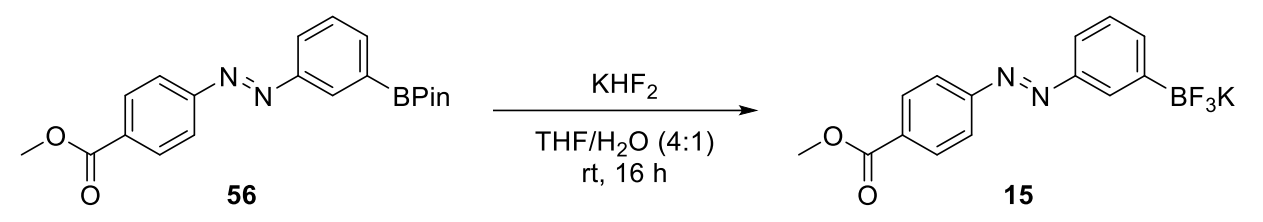

Figure S6. Two-step synthetic procedure for the synthesis of ester-containing PI 15.

Methyl (E)-4-((3-(trifluoro-14-boraneyl)phenyl)diazenyl)benzoate (potassium salt) 15 was synthesized in two steps as outlined in Fig. S6. In the first step, $(E)-4-((3-(4,4,5,5$-tetramethyl-1,3,2-dioxaborolan2-yl)phenyl)diazenyl)benzoic acid was methylated under acidic conditions using sulfuric acid as catalyst and methanol as methylating reagent/solvent. The potassium trifluoroborate-based PI 15 was obtained in the next step by reacting $\mathbf{5 6}$ with potassium bifluoride.

\subsubsection{Esterification of Benzoic Acid 50}

Methyl (E)-4-((3-(4,4,5,5-tetramethyl-1,3,2-dioxaborolan-2-yl)phenyl)diazenyl)benzoate (56)<smiles>COC(=O)c1ccc(N=Nc2cccc(B3OC(C)(C)C(C)(C)O3)c2)cc1</smiles>

Previously synthesized (E)-4-((3-(4,4,5,5-tetramethyl-1,3,2dioxaborolan-2-yl)phenyl)diazenyl) benzoic acid (130 mg, $0.37 \mathrm{mmol}, 1.0$ equiv) was dissolved in $\mathrm{MeOH}(20 \mathrm{~mL})$ and 10 drops of concentrated sulfuric acid were added. The stirred solution was refluxed at $70{ }^{\circ} \mathrm{C}$ for $16 \mathrm{~h}$ under an atmosphere of argon. The volatiles were removed and the residue was dissolved in DCM $(50 \mathrm{~mL})$. The organic layer was washed with $1 \mathrm{M}$ $\mathrm{NaOH}(50 \mathrm{~mL}), 1 \mathrm{M} \mathrm{HCl}(50 \mathrm{~mL})$ and brine $(50 \mathrm{~mL})$. The red organic solution was dried over anhydrous $\mathrm{MgSO}_{4}$, filtered and concentrated in vacuo. The crude reaction product was purified by column chromatography over silica gel (eluent $=$ DCM) to afford the pure methylated azobenzene boronate ester 56 as red solid (69 mg, $0.19 \mathrm{mmol}, 51 \%)$.

${ }^{1} \mathbf{H}$ NMR (400 MHz, $\left.\mathbf{C D C l}_{3}\right): \delta 8.39-8.36(\mathrm{~s}, 1 \mathrm{H}), 8.23-8.17(\mathrm{~m}, 2 \mathrm{H}), 8.03(\mathrm{ddd}, J=8.0,2.1,1.3$ $\mathrm{Hz}, 1 \mathrm{H}), 7.98-7.89(\mathrm{~m}, 3 \mathrm{H}), 7.54(\mathrm{ddd}, J=7.8,7.2,0.6 \mathrm{~Hz}, 1 \mathrm{H}), 3.96(\mathrm{~s}, 3 \mathrm{H}), 1.38(\mathrm{~s}, 12 \mathrm{H}) ;{ }^{13} \mathbf{C}\left\{{ }^{1} \mathbf{H}\right\}$ NMR (101 MHz, $\left.\mathbf{C D C l}_{3}\right): \delta 166.6,155.2,152.0,138.0,131.7,130.6,129.5,128.7,125.6,122.6,84.2$, $53.4,24.9$. The carbon directly attached to the boron atom was not detected due to quadrupolar broadening; ${ }^{11}$ B NMR (128 MHz, $\left.\mathbf{C D C l}_{3}\right)$ : $\delta 31.3$ (bs); $\mathbf{R}_{\mathbf{f}}$ (DCM): 0.61 . 


\subsubsection{Synthesis of Potassium Trifluoride-Based PI 15}

Methyl (E)-4-((3-(trifluoro-l4-boraneyl)phenyl)diazenyl)benzoate, potassium salt (15)<smiles>COC(=O)c1ccc(N=Nc2cccc(Br)c2)cc1</smiles>

Azobenzene trifluoroborate-based PI 15 was synthesized following general procedure GP3. In a polyethylene round-bottom flask, azobenzene boronate ester $56(69.0 \mathrm{mg}, 0.18 \mathrm{mmol}, 1.0$ equiv) was dissolved in a $4: 1$ mixture of THF and water $(10 \mathrm{~mL})$ and potassium bifluoride (42 mg, $0.54 \mathrm{mmol}, 3.0$ equiv) was added. After stirring at room temperature for $16 \mathrm{~h}$ (TLC indicated full consumption of starting material), $\mathrm{NaHCO}_{3}(22.6 \mathrm{mg}$, $0.27 \mathrm{mmol}, 1.5$ equiv) was added and the mixture was stirred for $10 \mathrm{~min}$. The volatiles were removed, the resulting oil was extracted with acetone $(20 \mathrm{~mL})$ and the extract was filtered. The solution was concentrated in vacuo. Pure potassium trifluoroborate PI $\mathbf{1 5}$ was obtained by recrystallisation from $\mathrm{Et}_{2} \mathrm{O} / n$-pentane as red-orange solid $(31.8 \mathrm{mg}, 0.09 \mathrm{mmol}, 51 \%)$.

${ }^{1}$ H NMR (400 MHz, DMSO-d $\left.\mathbf{d}_{\mathbf{6}}\right): \delta 8.17-8.12(\mathrm{~m}, 2 \mathrm{H}), 7.97(\mathrm{~d}, J=8.6 \mathrm{~Hz}, 2 \mathrm{H}), 7.91(\mathrm{~s}, 1 \mathrm{H}), 7.68-$ $7.63(\mathrm{~m}, 1 \mathrm{H}), 7.58-7.54(\mathrm{~m}, 1 \mathrm{H}), 7.38-7.31(\mathrm{~m}, 1 \mathrm{H}), 3.90(\mathrm{~s}, 3 \mathrm{H}) ;{ }^{13} \mathbf{C}\left\{{ }^{1} \mathbf{H}\right\}$ NMR (101 MHz, DMSO$\left.\boldsymbol{d}_{6}\right): \delta 165.7,154.8,151.0,135.9,130.9,130.5,127.4,125.4,125.0,122.4,120.6,52.4 ;{ }^{19} \mathbf{F}$ NMR (377 MHz, DMSO- $\left.\boldsymbol{d}_{6}\right): \delta-139.6$; ${ }^{11} \mathrm{~B}$ NMR (128 MHz, DMSO-d $\left.\boldsymbol{d}_{6}\right): \delta 3.4$ (bs); HRMS (ESI-): $\mathrm{m} / \mathrm{z}$ calculated for $\mathrm{C}_{14} \mathrm{H}_{11} \mathrm{BF}_{3} \mathrm{~N}_{2} \mathrm{O}_{2}^{-}\left(\left[\mathrm{M}-\mathrm{K}^{+}\right]^{-}\right)$307.0873, found 307.0867. 


\subsection{Synthesis of Potassium Trifluoroborate-Based PI 19}

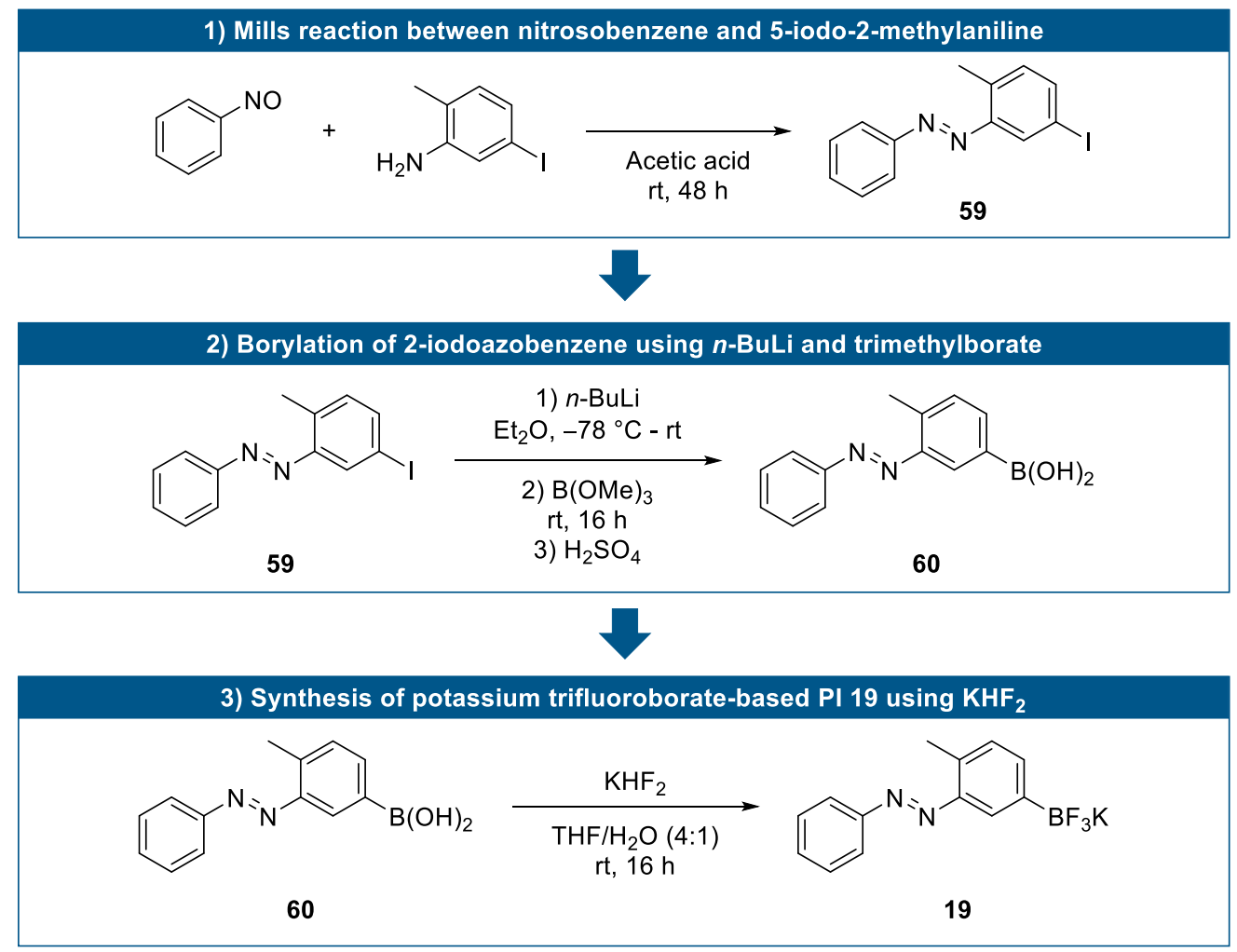

Figure S7. 3-Step synthetic procedure for the synthesis of potassium trifluoroborate-based PI 19.

(E)-1-(2-methyl-5-(trifluoro-14-boraneyl)phenyl)-2-phenyldiazene (potassium salt) 19 was synthesized in three steps as outlined in Fig. S7. In the first step, $(E)$-1-(5-iodo-2-methylphenyl)-2-phenyldiazene 59 was obtained via a Mills reaction between nitrosobenzene and 5-Iodo-2-methylaniline. Azobenzene 59 was then reacted in the second step to azobenzene boronic acid $\mathbf{6 0}$ using $n$-BuLi and trimethylborate (metal-halogen exchange). Finally, the boronic acid $\mathbf{6 0}$ was converted to the final potassium trifluoroborate-based PI 19 by the addition of potassium bifluoride.

\subsubsection{Mills Reaction to Obtain Iodoazobenzene 59}

\section{(E)-1-(5-iodo-2-methylphenyl)-2-phenyldiazene (59)}<smiles>Cc1ccc(I)cc1N=Nc1ccccc1</smiles>

Prepared from nitrosobenzene $(535 \mathrm{mg}, 5.0 \mathrm{mmol}, 1.0$ equiv) and 5-methyl-2iodoaniline $(1.165 \mathrm{~g}, 5.0 \mathrm{mmol}, 1.0$ equiv) following general procedure GP2 (stirred at $\mathrm{rt}$ for $48 \mathrm{~h}$ ). Purification by column chromatography over silica gel (eluent = DCM) afforded $\mathbf{5 9}$ as a red solid $(985 \mathrm{mg}, 3.1 \mathrm{mmol}, 61 \%)$.

${ }^{1} \mathbf{H}$ NMR (400 MHz, CDCl $): \delta 8.00(\mathrm{~d}, \mathrm{~J}=1.9 \mathrm{~Hz}, 1 \mathrm{H}), 7.98-7.93(\mathrm{~m}, 2 \mathrm{H}), 7.67(\mathrm{dd}, \mathrm{J}=8.0,1.9 \mathrm{~Hz}$, 1H), $7.57-7.47(\mathrm{~m}, 3 \mathrm{H}), 7.08(\mathrm{dd}, \mathrm{J}=8.0,0.8 \mathrm{~Hz}, 1 \mathrm{H}), 2.68(\mathrm{~s}, 3 \mathrm{H}) . ;{ }^{13} \mathbf{C}\left\{{ }^{1} \mathbf{H}\right\}$ NMR (101 MHz, $\left.\mathbf{C D C l}_{3}\right): \delta 152.7,151.2,139.3,137.8,133.0,131.3,129.2,124.7,123.2,91.5,17.3 ; \mathbf{R}_{\mathbf{f}}(\mathbf{D C M}): 0.84$. 


\subsubsection{Synthesis of Azobenzene Boronic Acid 60}

\section{(E)-(4-methyl-3-(phenyldiazenyl)phenyl)boronic acid (60)}

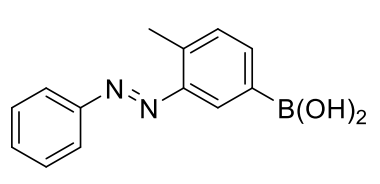

(E)-(4-methyl-3-(phenyldiazenyl)phenyl)boronic acid 60 was synthesized following a literature report from Kano et al. ${ }^{4}$ Under an argon atmosphere, $n$ $\operatorname{BuLi}(1.60 \mathrm{M}$ in hexane, $700 \mu \mathrm{L}, 1.1 \mathrm{mmol}, 1.1$ equiv) was added dropwise to a stirred $\mathrm{Et}_{2} \mathrm{O}$ solution $(10 \mathrm{~mL})$ of $(E)$-1-(5-iodo-2-methylphenyl)-2phenyldiazene $59\left(322 \mathrm{mg}, 1.0 \mathrm{mmol}, 1.0\right.$ equiv) at $-78{ }^{\circ} \mathrm{C}$. After the reaction solution was stirred for $30 \mathrm{~min}$ at $-78{ }^{\circ} \mathrm{C}$, an $\mathrm{Et}_{2} \mathrm{O}$ solution $(5 \mathrm{~mL})$ of trimethyl borate $(125 \mu \mathrm{L}, 1.1 \mathrm{mmol}, 1.1$ equiv) was added dropwise. The reaction temperature was gradually raised up to $\mathrm{rt}$ and the reaction mixture was stirred at room temperature for $16 \mathrm{~h}$. The yellow solution was quenched with water $(20 \mathrm{~mL})$ at $0{ }^{\circ} \mathrm{C}$ and $2 \mathrm{M}$ sulfuric acid $(2.5 \mathrm{~mL})$ was added. The organic layer was separated and the aqueous layer was extracted with $\mathrm{Et}_{2} \mathrm{O}(3 \times 30 \mathrm{~mL})$. The combined organic layers were treated with aq. $1 \mathrm{M} \mathrm{NaOH}(10 \mathrm{~mL})$, washed with $\mathrm{Et}_{2} \mathrm{O}(3 \times 50 \mathrm{~mL})$ and neutralized with $2 \mathrm{M}$ sulfuric acid. The precipitated yellow solid was dissolved in $\mathrm{Et}_{2} \mathrm{O}(30 \mathrm{~mL})$, the organic layer was separated, dried over anhydrous $\mathrm{MgSO}_{4}$, filtered and concentrated in vacuo. The residue was recrystallized from benzene to give boronic acid $\mathbf{6 0}$ as orange solid (104.2 mg, $0.43 \mathrm{mmol}, 43 \%)$.

${ }^{1} \mathbf{H}$ NMR (400 MHz, CDCl $): \delta 8.43(\mathrm{~d}, \mathrm{~J}=1.2 \mathrm{~Hz}, 1 \mathrm{H}), 8.22(\mathrm{dd}, \mathrm{J}=7.5,1.3 \mathrm{~Hz}, 1 \mathrm{H}), 8.07-7.92(\mathrm{~m}$, 2H), $7.51-7.43(\mathrm{~m}, 3 \mathrm{H}), 7.34(\mathrm{~d}, \mathrm{~J}=7.6 \mathrm{~Hz}, 1 \mathrm{H}), 2.75$ (s, 3H); ${ }^{13} \mathbf{C}\left\{{ }^{1} \mathbf{H}\right\}$ NMR (101 MHz, CDCl $\left.\mathbf{3}\right): \delta$ $153.4,150.1,142.9,138.1,131.7,130.1,129.5,123.7,122.3,18.5$. The carbon directly attached to the boron atom was not detected due to quadrupolar broadening; $\left.{ }^{11} \mathbf{B} \mathbf{~ N M R ~ ( 1 2 8 ~} \mathbf{M H z}, \mathbf{C D C l}_{3}\right): \delta 30.9$ (bs).

\subsubsection{Synthesis of Potassium Trifluoride-Based PI 19}

\section{(E)-1-(2-methyl-5-(trifluoro-14-boraneyl)phenyl)-2-phenyldiazene (potassium salt) (19)}

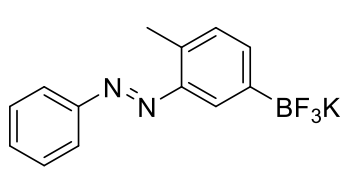

Azobenzene trifluoroborate $\mathbf{1 9}$ was synthesized following general procedure GP3. In a polyethylene round-bottom flask, azobenzene boronic acid $\mathbf{6 0}$ (84.1 mg, $0.35 \mathrm{mmol}, 1.0$ equiv) was dissolved in a 4:1 mixture of THF and water $(10 \mathrm{~mL})$ and potassium bifluoride $(82.1 \mathrm{mg}, 1.05 \mathrm{mmol}, 3.0$ equiv) was added. After stirring at room temperature for $16 \mathrm{~h}$ (TLC indicated full consumption of starting material), $\mathrm{NaHCO}_{3}$ (44.1 mg, $0.53 \mathrm{mmol}, 1.5$ equiv) was added and the mixture was stirred for $10 \mathrm{~min}$. The volatiles were removed, the resulting oil was extracted with acetone $(20 \mathrm{~mL})$ and the extract was filtered. The solution was concentrated in vacuo. Pure potassium trifluoroborate PI 19 was obtained by recrystallisation from $\mathrm{Et}_{2} \mathrm{O} / \mathrm{n}$-pentane as orange solid (51.3 $\mathrm{mg}, 0.17 \mathrm{mmol}, 49 \%$ ).

${ }^{1} \mathbf{H}$ NMR (400 MHz, CDCl $): \delta 8.40(\mathrm{~d}, \mathrm{~J}=1.2 \mathrm{~Hz}, 1 \mathrm{H}), 8.17(\mathrm{dd}, \mathrm{J}=7.5,1.3 \mathrm{~Hz}, 1 \mathrm{H}), 8.01-7.97(\mathrm{~m}$, 2H), $7.55-7.51(\mathrm{~m}, 3 \mathrm{H}), 7.43(\mathrm{~d}, \mathrm{~J}=7.6 \mathrm{~Hz}, 1 \mathrm{H}), 2.77(\mathrm{~s}, 3 \mathrm{H}) ;{ }^{13} \mathbf{C}\left\{{ }^{1} \mathbf{H}\right\}$ NMR (101 MHz, CDCl $): \delta$ 153.1, 150.5, 142.6, 137.8, 131.1, 130.8, 129.1, 123.1, 122.7, 119.4, 18.0; ${ }^{19} \mathbf{F}$ NMR (377 $\left.\mathbf{~ M H z , ~} \mathbf{C D C l}_{3}\right)$ : $\delta-139.2 ;{ }^{11} \mathbf{B}$ NMR $\left(\mathbf{1 2 8} \mathbf{M H z}, \mathbf{C D C l}_{3}\right): \delta 3.5(\mathrm{bs})$; HRMS (ESI-): $\mathrm{m} / z$ calculated for $\mathrm{C}_{13} \mathrm{H}_{11} \mathrm{BF}_{3} \mathrm{~N}_{2}{ }^{-}$ $\left(\left[\mathrm{M}-\mathrm{K}^{+}\right]^{-}\right)$263.0975, found 263.0970. 


\section{Photophysical Characterization of PIs}

\subsection{UV/vis Absorption Studies of PIs}

$\mathrm{UV/vis} \mathrm{absorption} \mathrm{spectroscopy} \mathrm{has} \mathrm{been} \mathrm{performed} \mathrm{using} \mathrm{a} \mathrm{Jasco} \mathrm{V-650} \mathrm{spectrophotometer,} \mathrm{equipped}$ with a temperature control unit at $25^{\circ} \mathrm{C}$. The samples were measured in Hellma QS quartz cuvettes (chamber volume $=1.4 \mathrm{~mL}, \mathrm{H} \times \mathrm{W} \times \mathrm{D}=46 \mathrm{~mm} \times 12.5 \mathrm{~mm}, 12.5 \mathrm{~mm}$ ) fitted with a PTFE stopper. For the UV/vis spectra determination of the trans-isomer, a $5 \times 10^{-5} \mathrm{M}$ solution of the respective PI in a Tris/Ca ${ }^{2+}(200 \mathrm{mM} / 20 \mathrm{mM}, \mathrm{pH}=7.8)$ buffer (or DMSO in case of limited water solubility of the compound) was prepared. Afterwards, the trans-PI solution was irradiated within the cuvette for $60 \mathrm{sec}$ using a UV-A LED $\left(3 \mathrm{~W}, \lambda_{\max }=365 \mathrm{~nm}\right.$ ) to record the UV/vis spectra of the compound at the PSS at $365 \mathrm{~nm}$. The corresponding UV/vis spectra are displayed below.

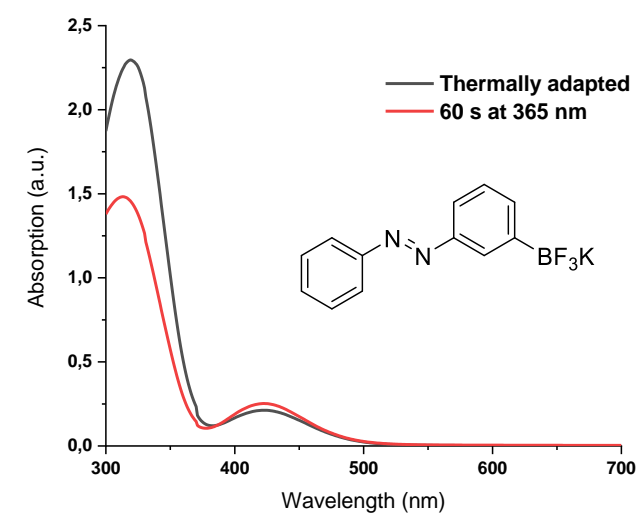

Figure S8. UV/vis spectra of photo-isomerization of $50 \mu \mathrm{M}$ PI 1 in Tris/Ca ${ }^{2+}$ buffer upon $365 \mathrm{~nm}$ irradiation.

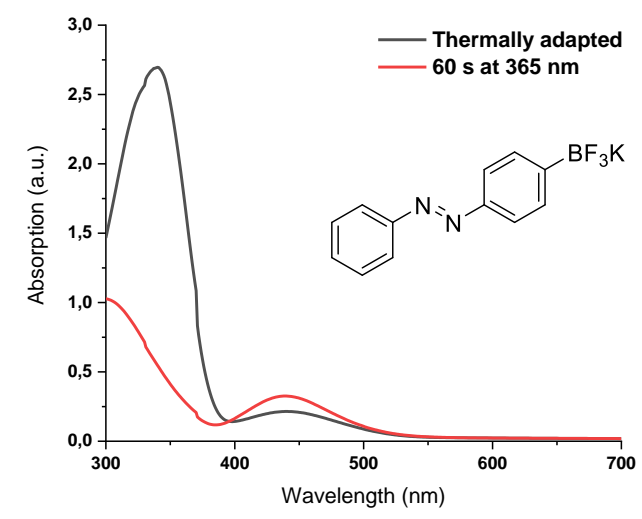

Figure S9. UV/vis spectra of photo-isomerization of $50 \mu \mathrm{M}$ PI 2 in Tris/Ca ${ }^{2+}$ buffer upon $365 \mathrm{~nm}$ irradiation. 


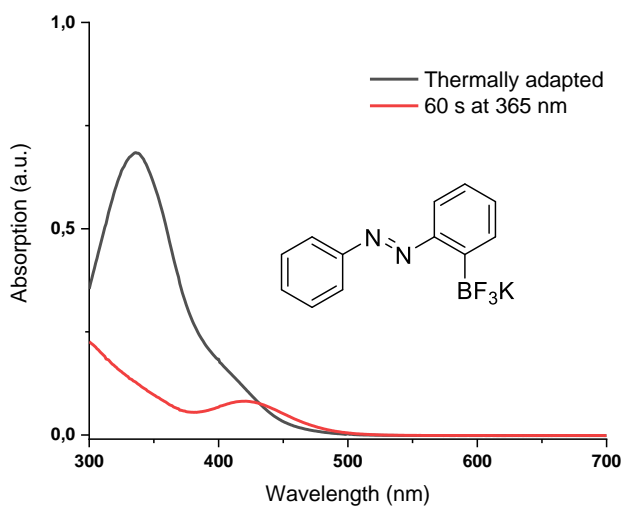

Figure S10. UV/vis spectra of photo-isomerization of $50 \mu \mathrm{M}$ PI 3 in Tris/Ca ${ }^{2+}$ buffer upon $365 \mathrm{~nm}$ irradiation.

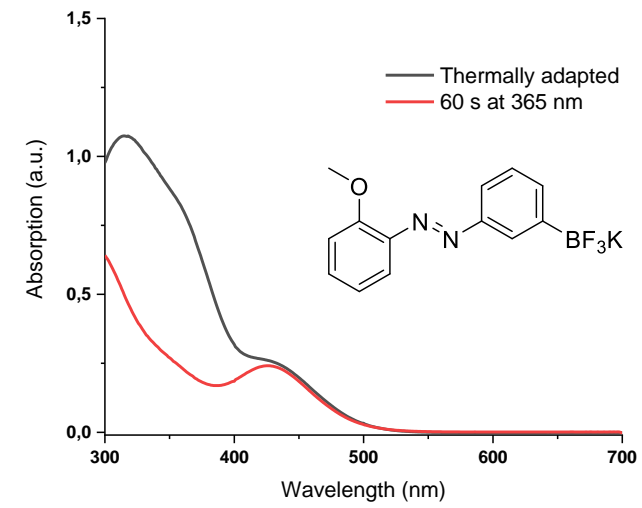

Figure S11. UV/vis spectra of photo-isomerization of $50 \mu \mathrm{M}$ PI 4 in Tris/Ca ${ }^{2+}$ buffer upon $365 \mathrm{~nm}$ irradiation.

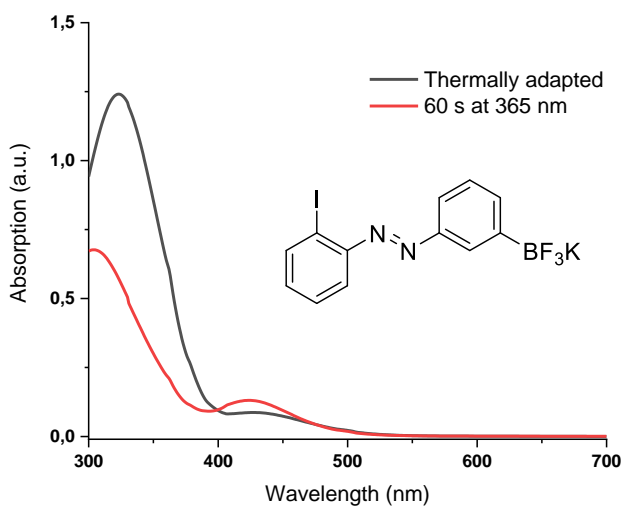

Figure S12. UV/vis spectra of photo-isomerization of $50 \mu \mathrm{M}$ PI 5 in Tris/Ca ${ }^{2+}$ buffer upon $365 \mathrm{~nm}$ irradiation. 


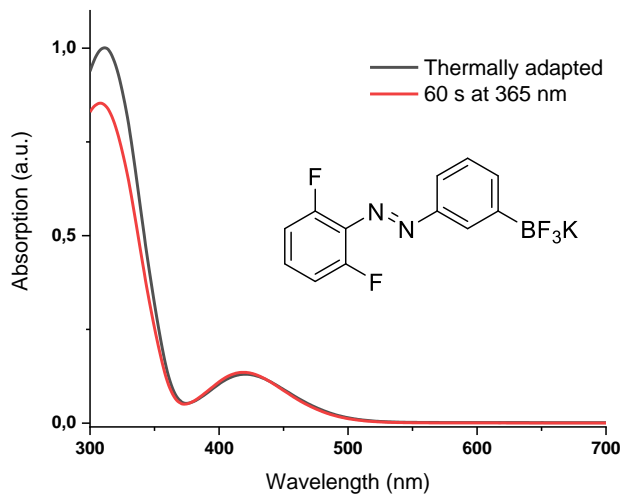

Figure S13. UV/vis spectra of photo-isomerization of $50 \mu \mathrm{M}$ PI 6 in Tris/Ca ${ }^{2+}$ buffer upon $365 \mathrm{~nm}$ irradiation.

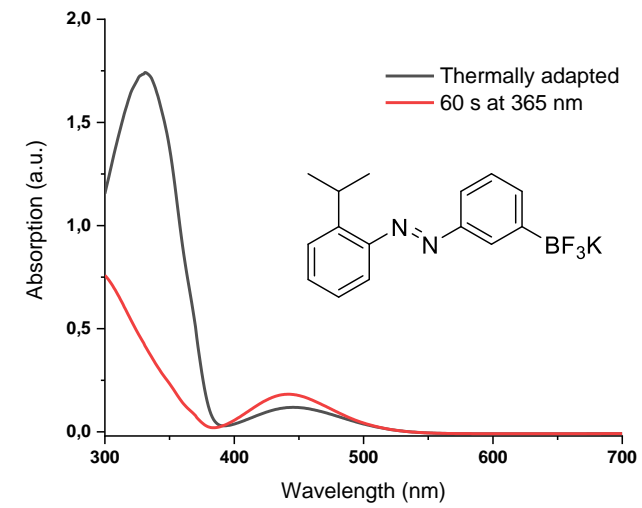

Figure S14. UV/vis spectra of photo-isomerization of $50 \mu$ M PI 7 in Tris/Ca ${ }^{2+}$ buffer upon $365 \mathrm{~nm}$ irradiation.

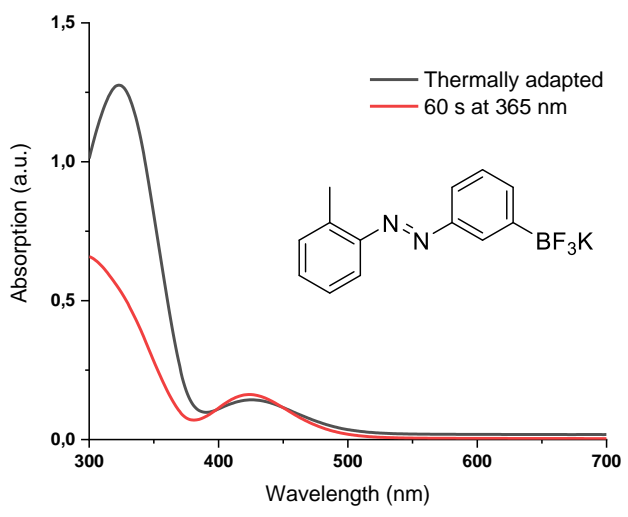

Figure S15. UV/vis spectra of photo-isomerization of $50 \mu \mathrm{M}$ PI 8 in Tris/Ca ${ }^{2+}$ buffer upon $365 \mathrm{~nm}$ irradiation. 


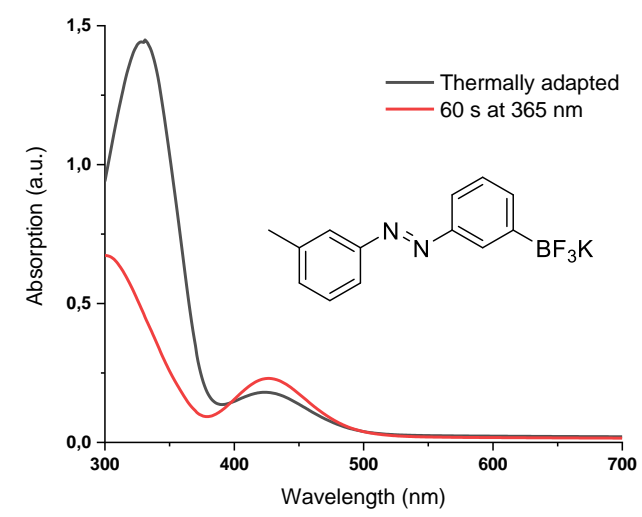

Figure S16. UV/vis spectra of photo-isomerization of $50 \mu \mathrm{M}$ PI 9 in Tris/Ca ${ }^{2+}$ buffer upon $365 \mathrm{~nm}$ irradiation.

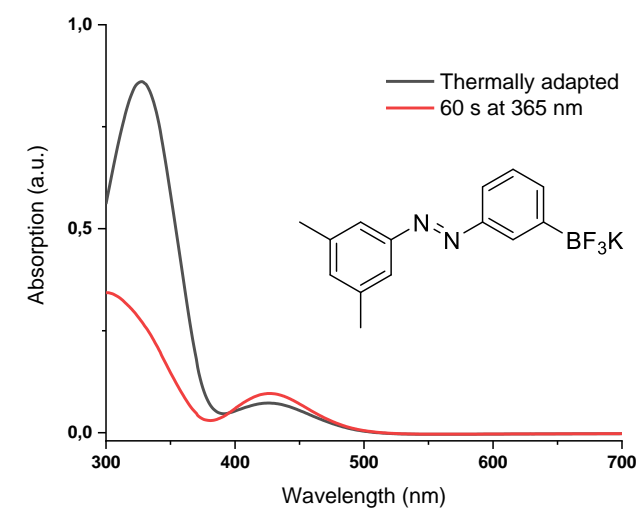

Figure S17. UV/vis spectra of photo-isomerization of $50 \mu \mathrm{M}$ PI 10 in DMSO upon $365 \mathrm{~nm}$ irradiation.

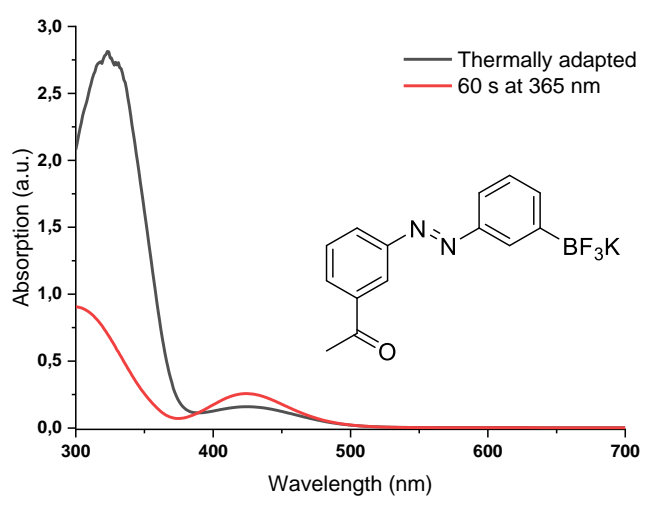

Figure S18. UV/vis spectra of photo-isomerization of $50 \mu \mathrm{M}$ PI 11 in Tris/ $\mathrm{Ca}^{2+}$ buffer upon $365 \mathrm{~nm}$ irradiation. 


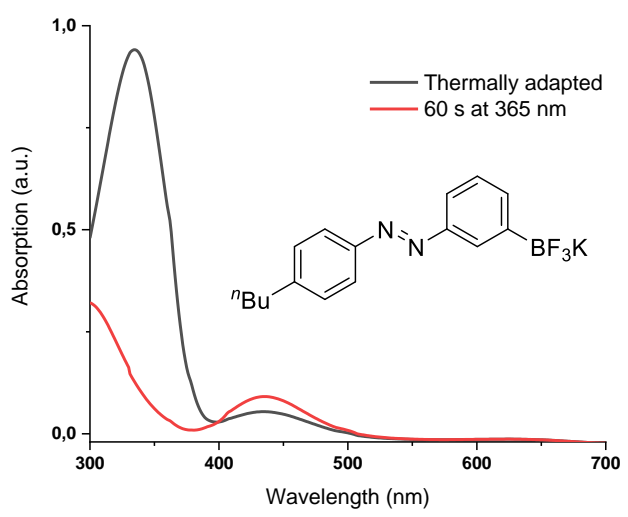

Figure S19. UV/vis spectra of photo-isomerization of $50 \mu \mathrm{M}$ PI 12 in DMSO upon $365 \mathrm{~nm}$ irradiation.

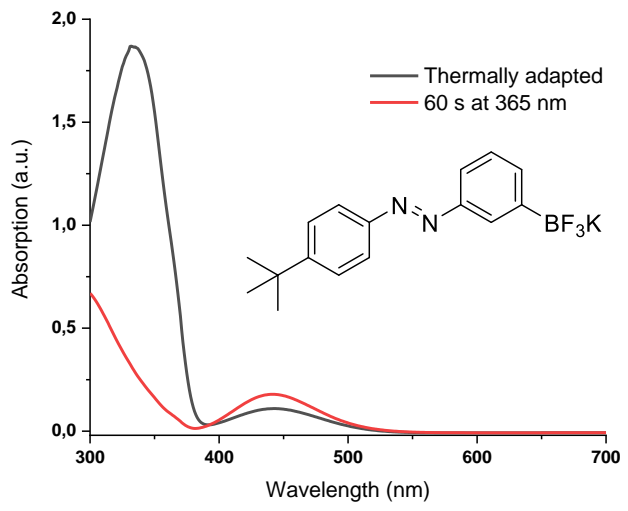

Figure S20. UV/vis spectra of photo-isomerization of $50 \mu \mathrm{M}$ PI 13 in DMSO upon $365 \mathrm{~nm}$ irradiation.

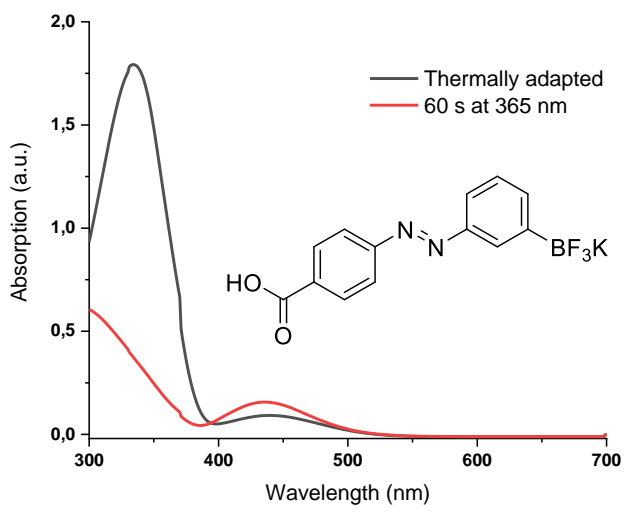

Figure S21. UV/vis spectra of photo-isomerization of $50 \mu \mathrm{M}$ PI 14 in Tris/Ca ${ }^{2+}$ buffer upon $365 \mathrm{~nm}$ irradiation. 


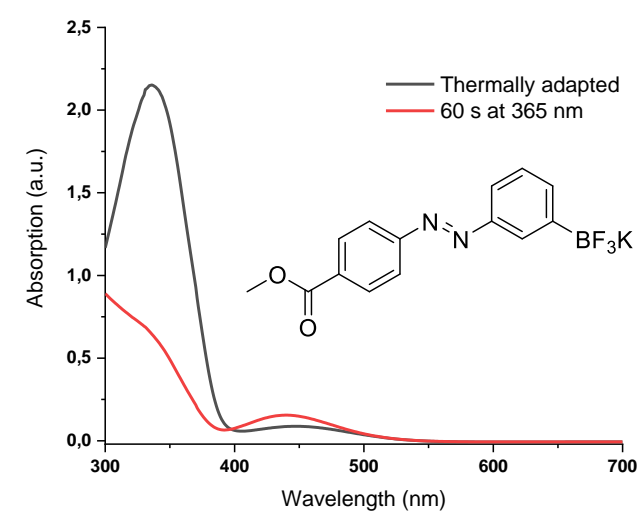

Figure S22. UV/vis spectra of photo-isomerization of $50 \mu \mathrm{M}$ PI 15 in DMSO upon $365 \mathrm{~nm}$ irradiation.

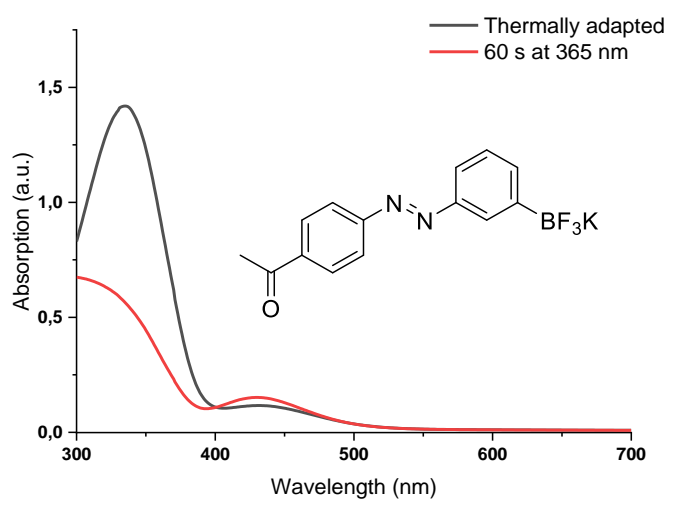

Figure S23. UV/vis spectra of photo-isomerization of $50 \mu \mathrm{M}$ PI 16 in Tris/Ca ${ }^{2+}$ buffer upon $365 \mathrm{~nm}$ irradiation.

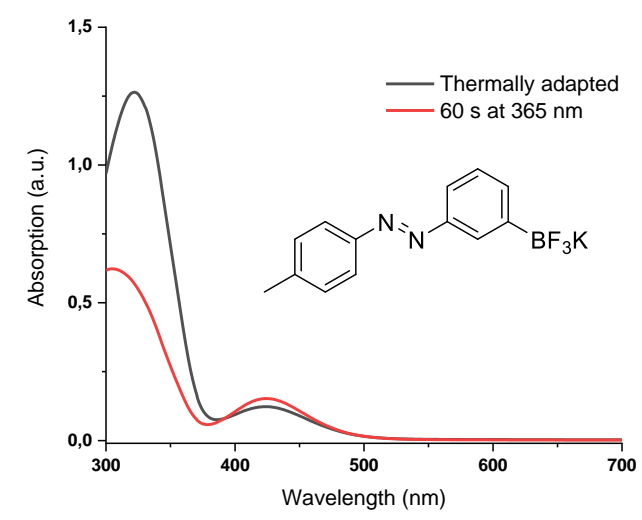

Figure S24. UV/vis spectra of photo-isomerization of $50 \mu \mathrm{M}$ PI 17 in Tris/ $\mathrm{Ca}^{2+}$ buffer upon $365 \mathrm{~nm}$ irradiation. 


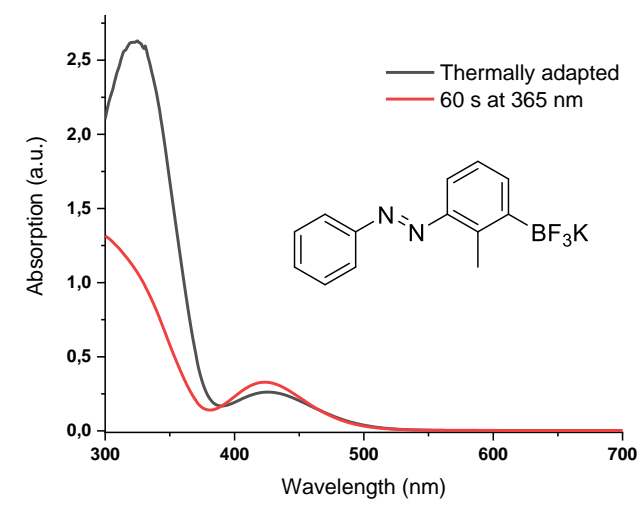

Figure S25. UV/vis spectra of photo-isomerization of $50 \mu \mathrm{M}$ PI 18 in Tris/Ca ${ }^{2+}$ buffer upon $365 \mathrm{~nm}$ irradiation.

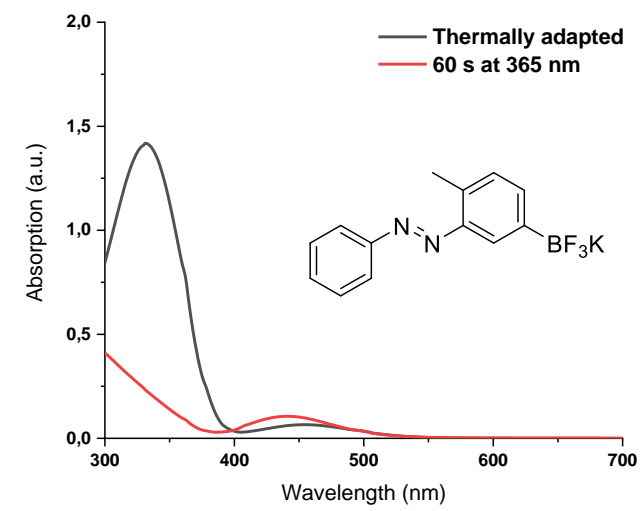

Figure S26. UV/vis spectra of photo-isomerization of $50 \mu \mathrm{M}$ PI 19 in Tris/Ca ${ }^{2+}$ buffer upon $365 \mathrm{~nm}$ irradiation.

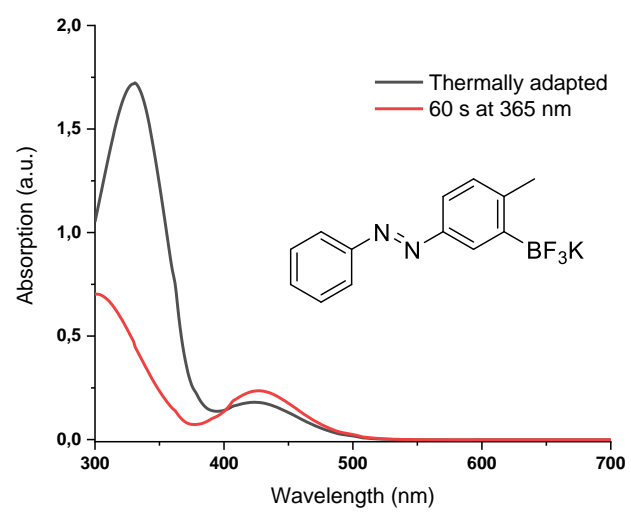

Figure S27. UV/vis spectra of photo-isomerization of $50 \mu \mathrm{M}$ PI 20/PI in Tris/ $\mathrm{Ca}^{2+}$ buffer upon $365 \mathrm{~nm}$ irradiation. 


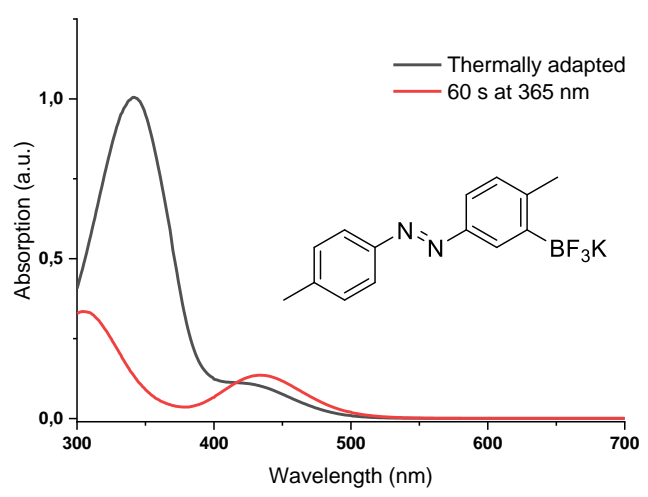

Figure S28. UV/vis spectra of photo-isomerization of $50 \mu \mathrm{M}$ PI 21 in Tris/Ca ${ }^{2+}$ buffer upon $365 \mathrm{~nm}$ irradiation. 


\subsection{Photoreversibility and -stability of PIs}

The analysis of the photoreversibility and photostability of PIs has been performed using a Jasco V-650 spectrophotometer, equipped with a temperature control unit at $25^{\circ} \mathrm{C}$. The samples were measured in Hellma QS quartz cuvettes (chamber volume $=1.4 \mathrm{~mL}, \mathrm{H} \times \mathrm{W} \times \mathrm{D}=46 \mathrm{~mm} \times 12.5 \mathrm{~mm}, 12.5 \mathrm{~mm}$ ) fitted with a PTFE stopper. For the UV/vis spectra determination of the thermally adapted trans-isomer, a $5 \times 10^{-5} \mathrm{M}$ solution of the respective PI in a Tris/ $\mathrm{Ca}^{2+}(200 \mathrm{mM} / 20 \mathrm{mM}, \mathrm{pH}=7.8)$ buffer (or DMSO in case of limited water solubility of the compound) was prepared. After the thermally adapted UV/vis spectra has been recorded, the PI solution was irradiated within the cuvette for $60 \mathrm{sec}$ with UV-A light ( $3 \mathrm{~W}, \lambda_{\max }=365 \mathrm{~nm}$ ) and the UV/vis spectra was recorded. Next, the solution within the cuvette was irradiated with blue light $\left(5 \mathrm{~W}, \lambda_{\max }=460 \mathrm{~nm}\right.$ ) for $60 \mathrm{sec}$ and the UV/vis spectra was measured. A labmade irradiation setup was constructed ensuring the same distance between light source and cuvette for all measurements. This process was repeated five times to get critical data on the judgement of the PI's photoreversibility and -stability, which is required for later applications. The results of this analysis are outlined below.

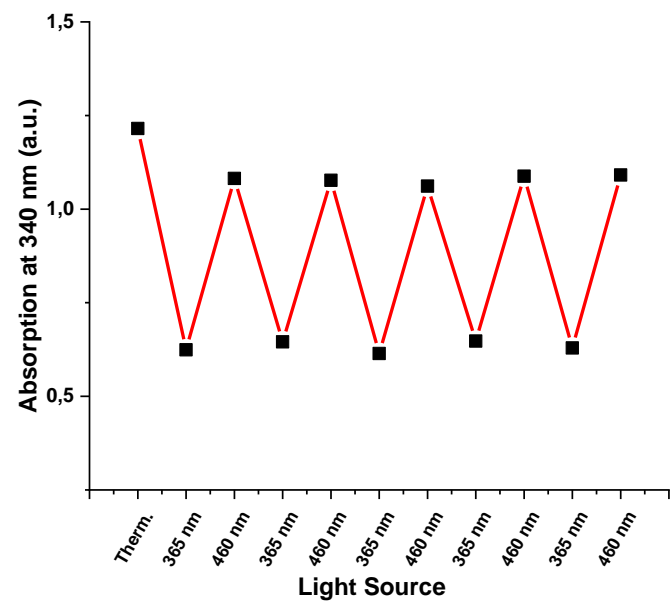<smiles>[Mg]C(Br)(Br)c1cccc(/N=N/c2ccccc2)c1</smiles>

Figure S29. Photoreversibility of PI 1 in Tris/Ca ${ }^{2+}$ buffer $(50 \mu \mathrm{M})$ at successive irradiation cycles of 365/460 nm.
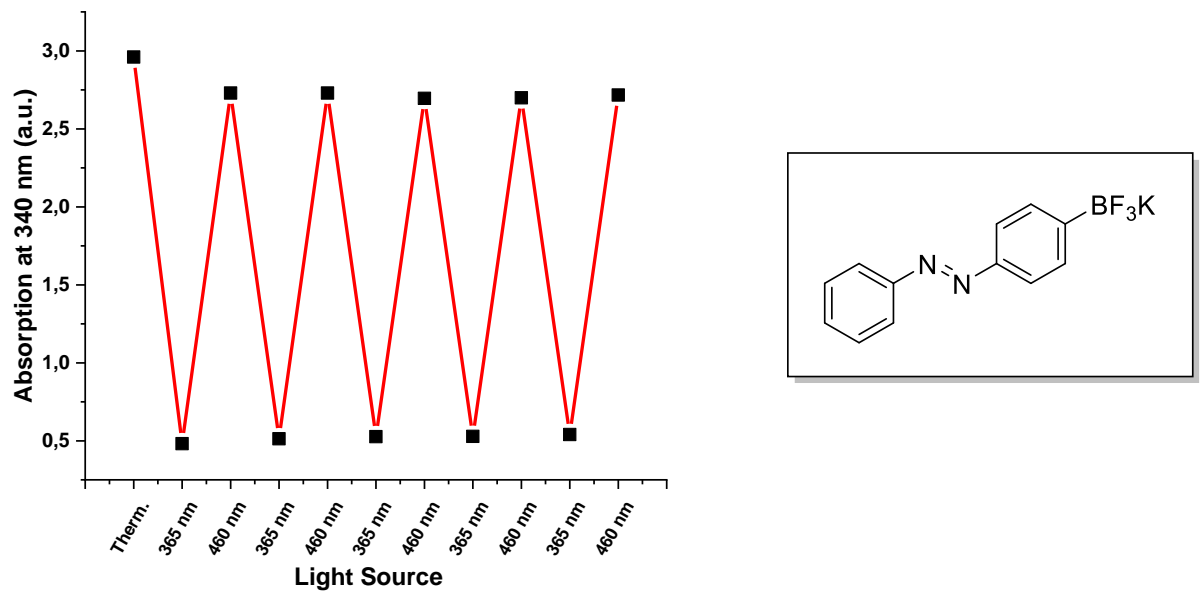

Figure S30. Photoreversibility of PI 2 in Tris/Ca ${ }^{2+}$ buffer $(50 \mu \mathrm{M})$ at successive irradiation cycles of $365 / 460 \mathrm{~nm}$. 


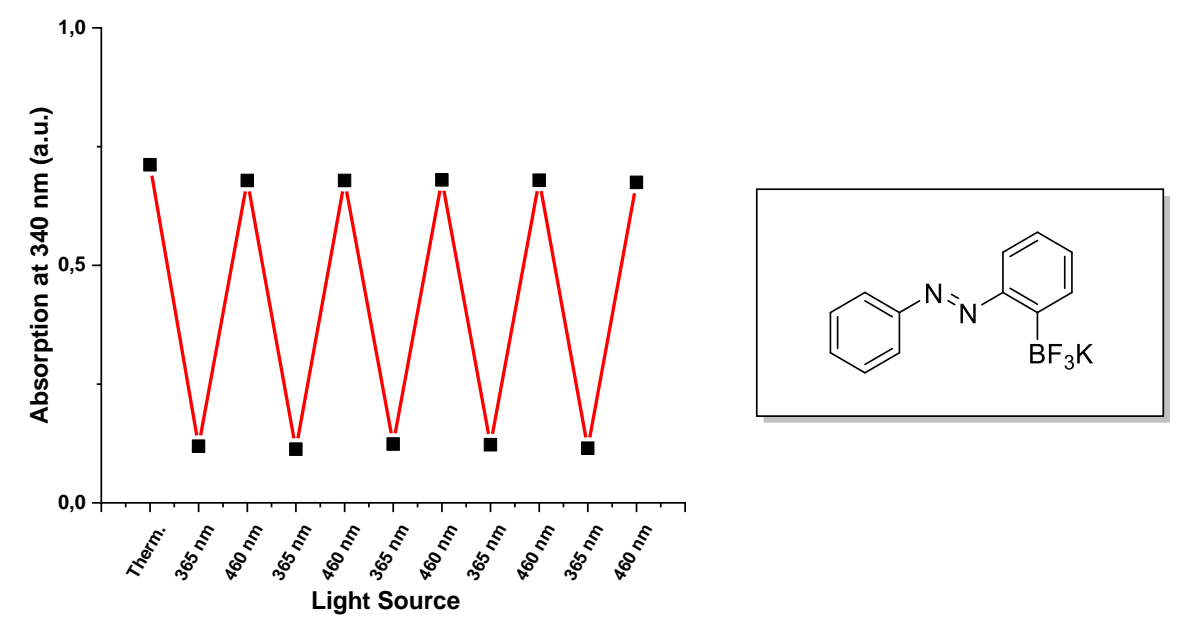

Figure S31. Photoreversibility of PI 3 in Tris/Ca ${ }^{2+}$ buffer $(50 \mu \mathrm{M})$ at successive irradiation cycles of $365 / 460 \mathrm{~nm}$.
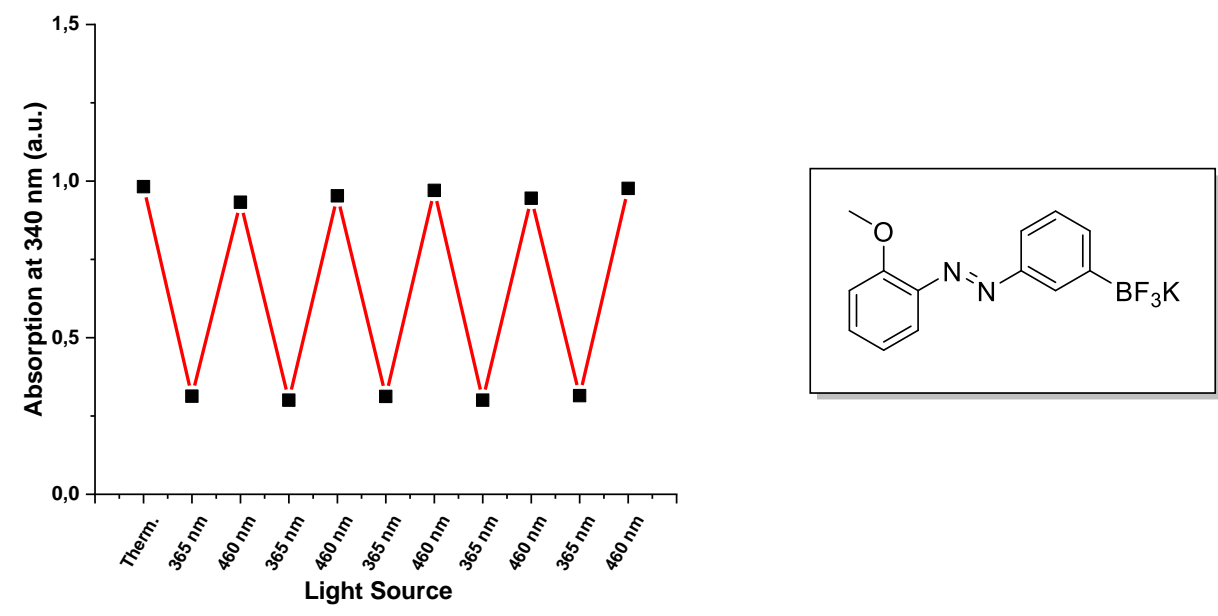

Figure S32. Photoreversibility of PI 4 in Tris/Ca ${ }^{2+}$ buffer $(50 \mu \mathrm{M})$ at successive irradiation cycles of $365 / 460 \mathrm{~nm}$.

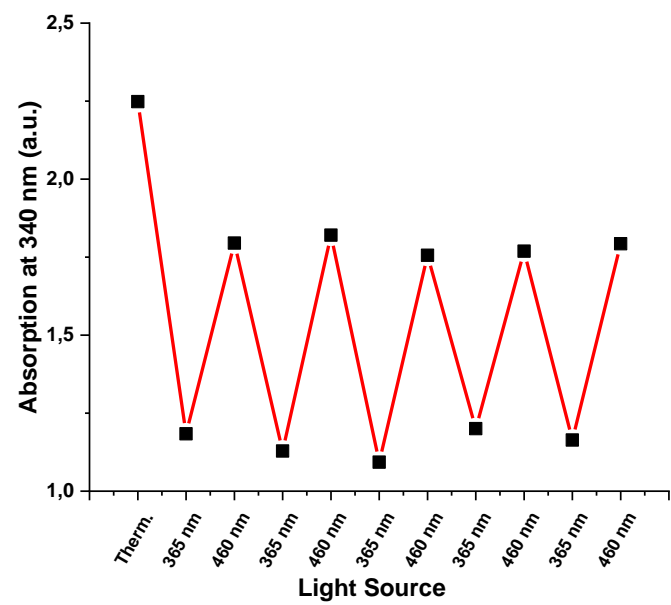<smiles>[X]C(Br)(Br)c1cccc(N=Nc2ccccc2I)c1</smiles>

Figure S33. Photoreversibility of PI 5 in Tris/Ca ${ }^{2+}$ buffer $(50 \mu \mathrm{M})$ at successive irradiation cycles of $365 / 460 \mathrm{~nm}$. 

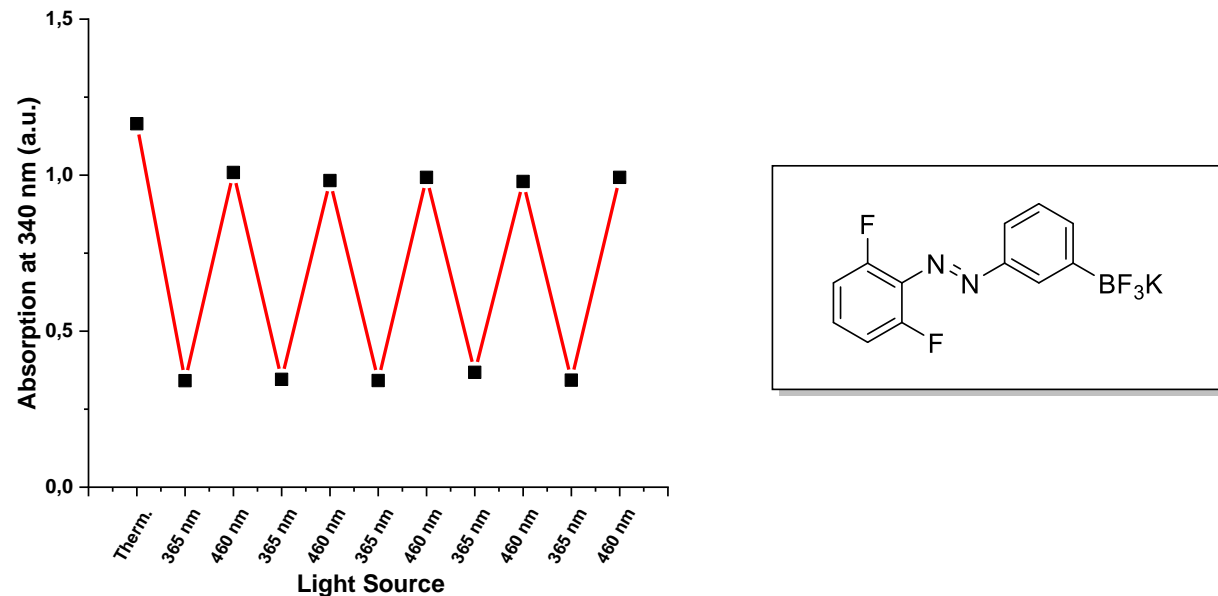

Figure S34. Photoreversibility of PI 6 in Tris/Ca ${ }^{2+}$ buffer $(50 \mu \mathrm{M})$ at successive irradiation cycles of 365/460 $\mathrm{nm}$.
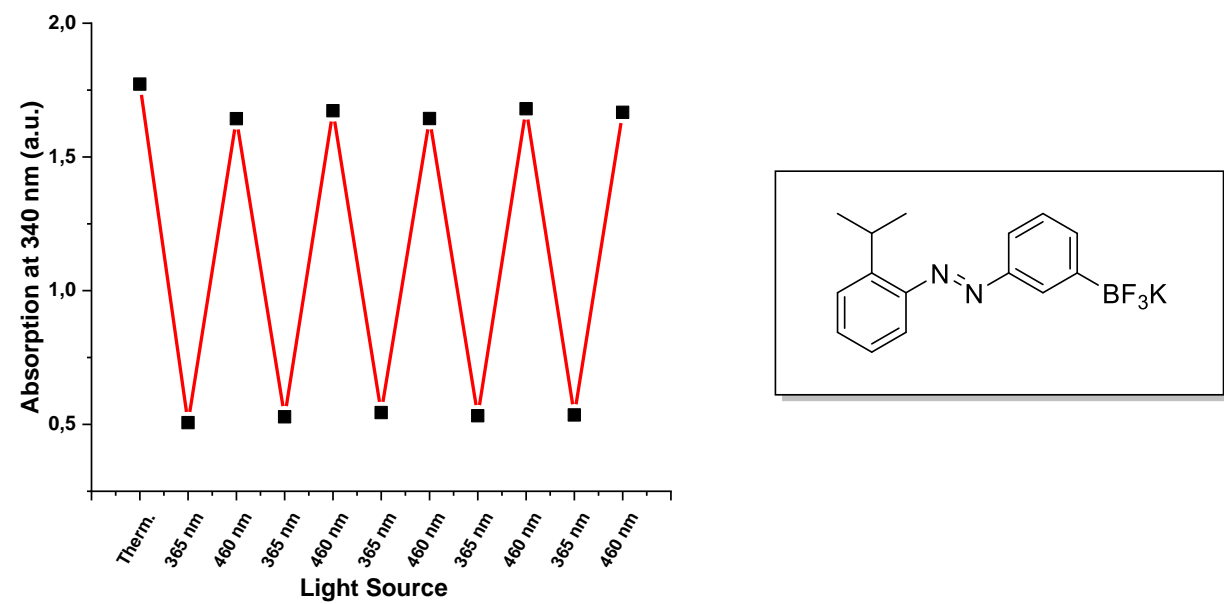

Figure S35. Photoreversibility of PI 7 in Tris/Ca ${ }^{2+}$ buffer $(50 \mu \mathrm{M})$ at successive irradiation cycles of 365/460 $\mathrm{nm}$.
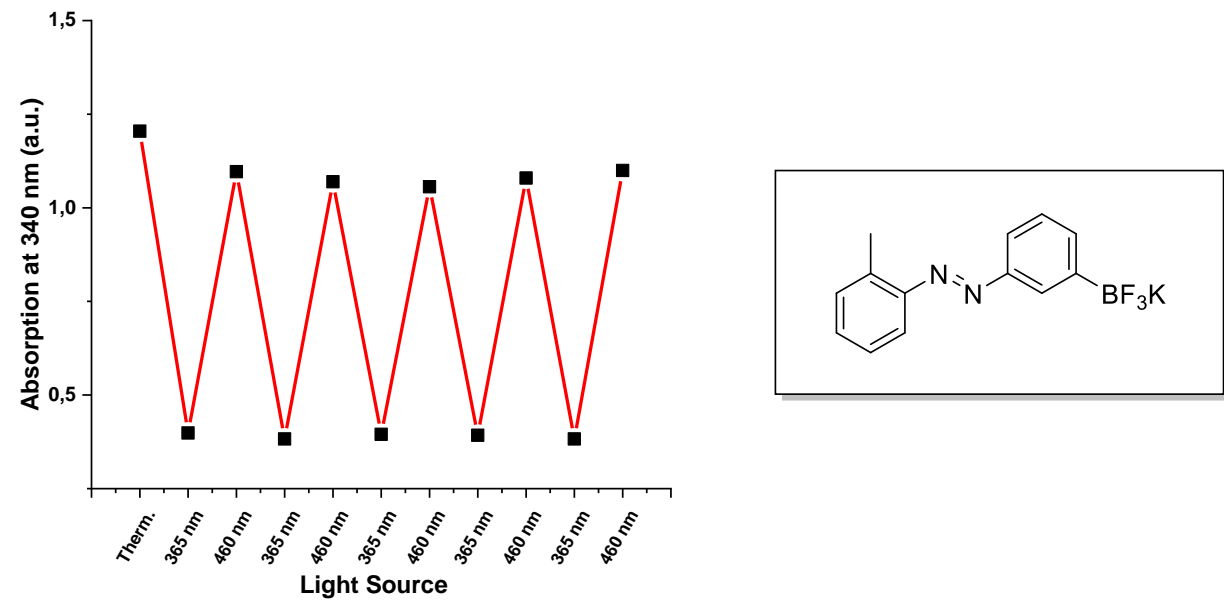

Figure S36. Photoreversibility of PI 8 in Tris/Ca ${ }^{2+}$ buffer $(50 \mu \mathrm{M})$ at successive irradiation cycles of $365 / 460 \mathrm{~nm}$. 


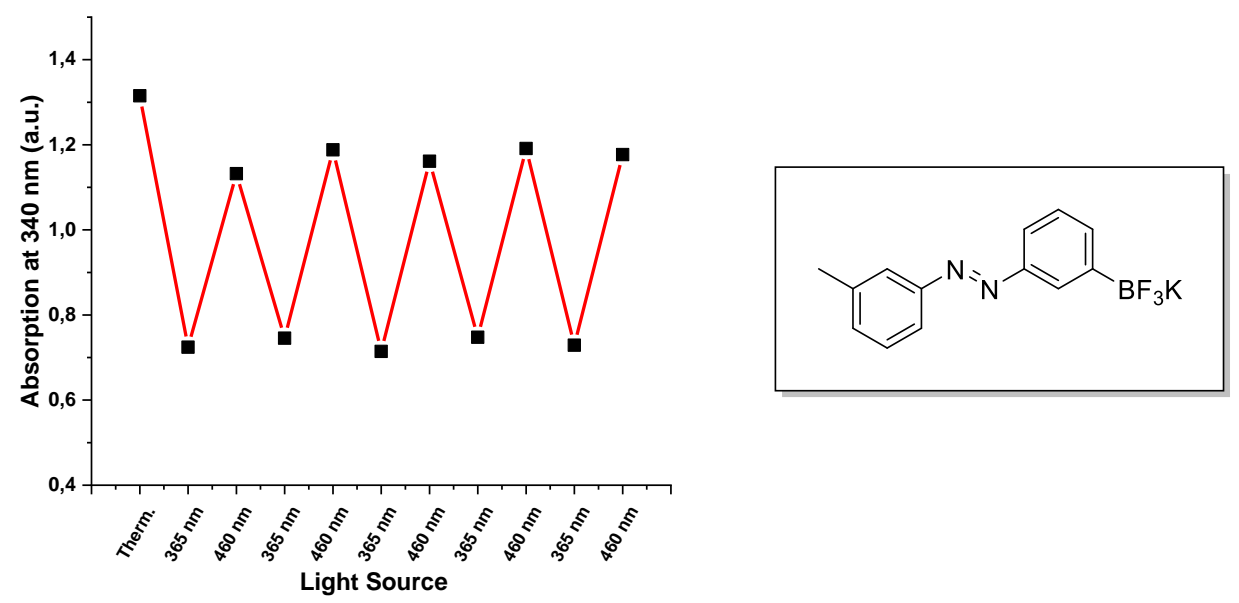

Figure S37. Photoreversibility of PI 9 in Tris/Ca ${ }^{2+}$ buffer $(50 \mu \mathrm{M})$ at successive irradiation cycles of $365 / 460 \mathrm{~nm}$.
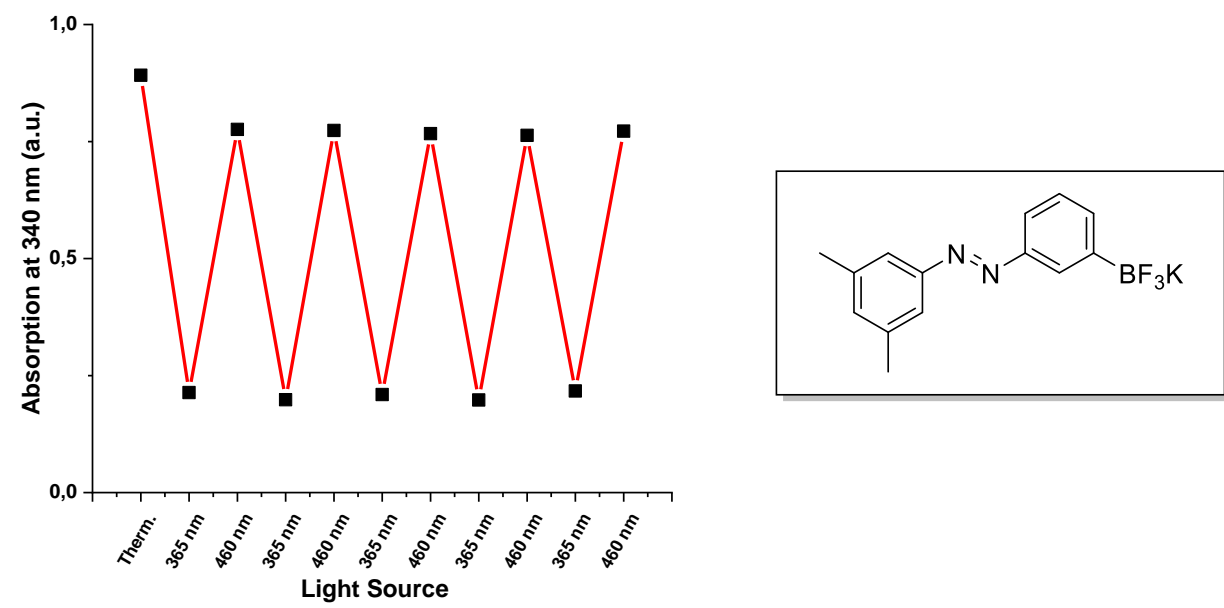

Figure S38. Photoreversibility of PI 10 in DMSO $(50 \mu \mathrm{M})$ at successive irradiation cycles of 365/460 nm.
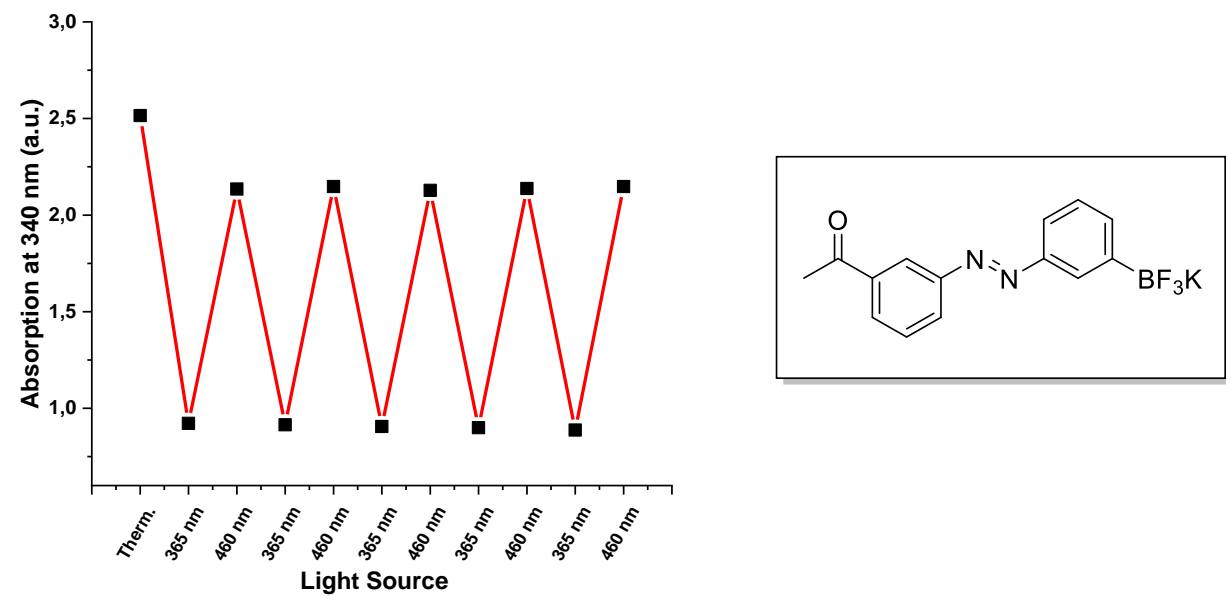

Figure S39. Photoreversibility of PI 11 in Tris/ $\mathrm{Ca}^{2+}$ buffer $(50 \mu \mathrm{M})$ at successive irradiation cycles of 365/460 nm. 

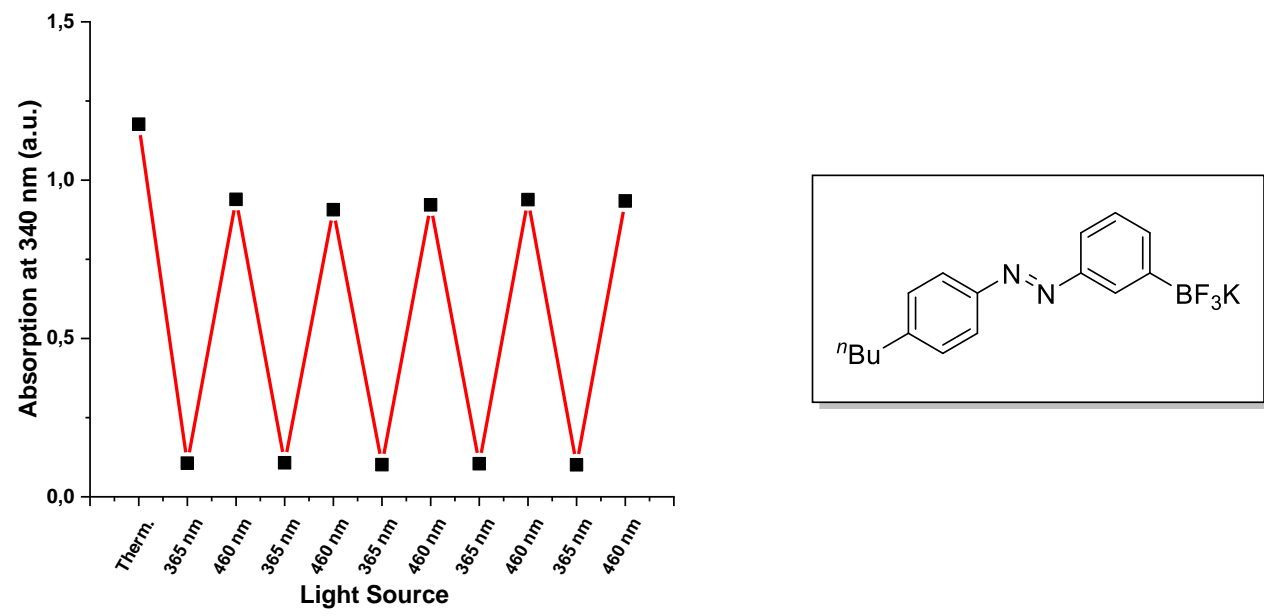

Figure S40. Photoreversibility of PI 12 in DMSO $(50 \mu \mathrm{M})$ at successive irradiation cycles of 365/460 nm.
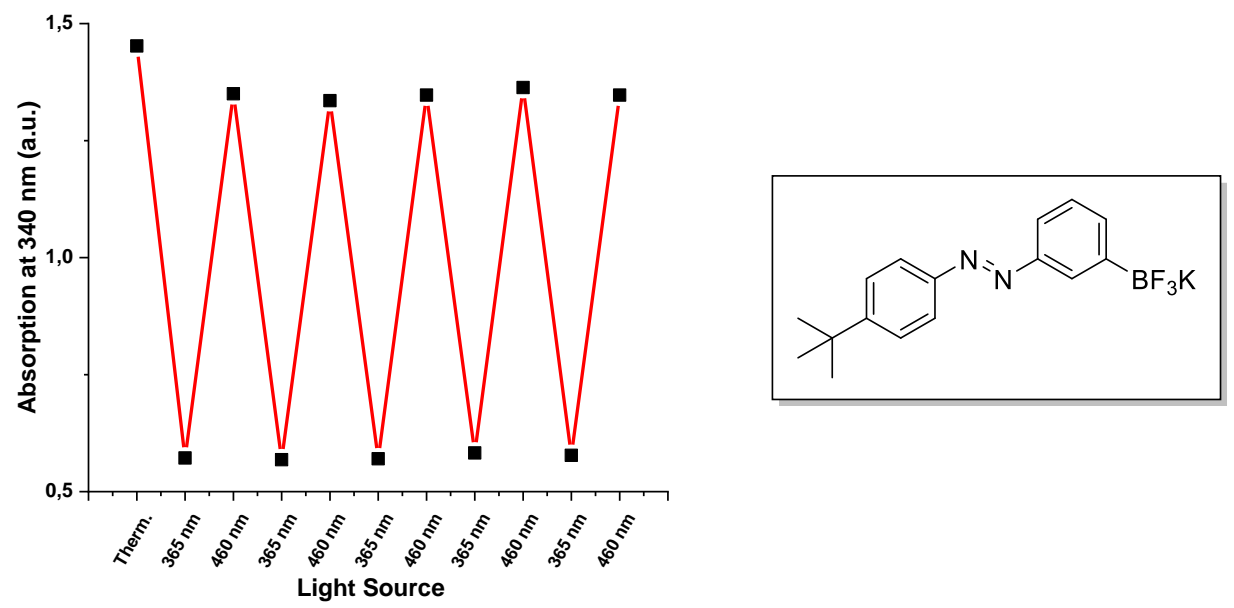

Figure S41. Photoreversibility of PI 13 in DMSO $(50 \mu \mathrm{M})$ at successive irradiation cycles of 365/460 nm.
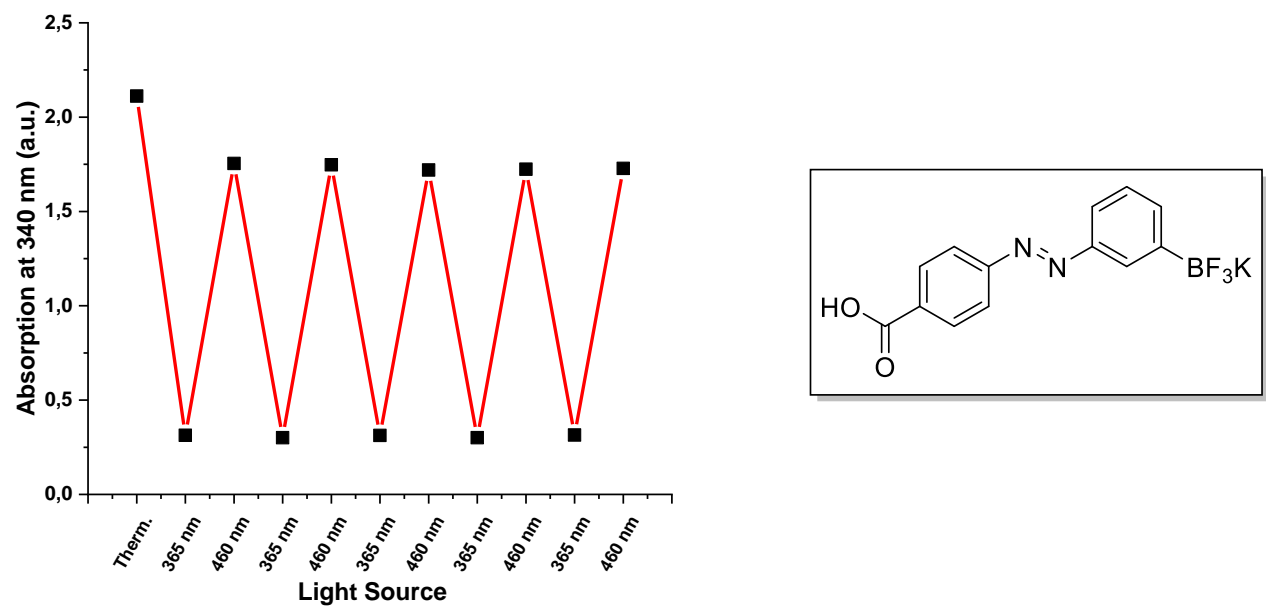

Figure S42. Photoreversibility of PI 14 in Tris/ $\mathrm{Ca}^{2+}$ buffer $(50 \mu \mathrm{M})$ at successive irradiation cycles of 365/460 nm. 


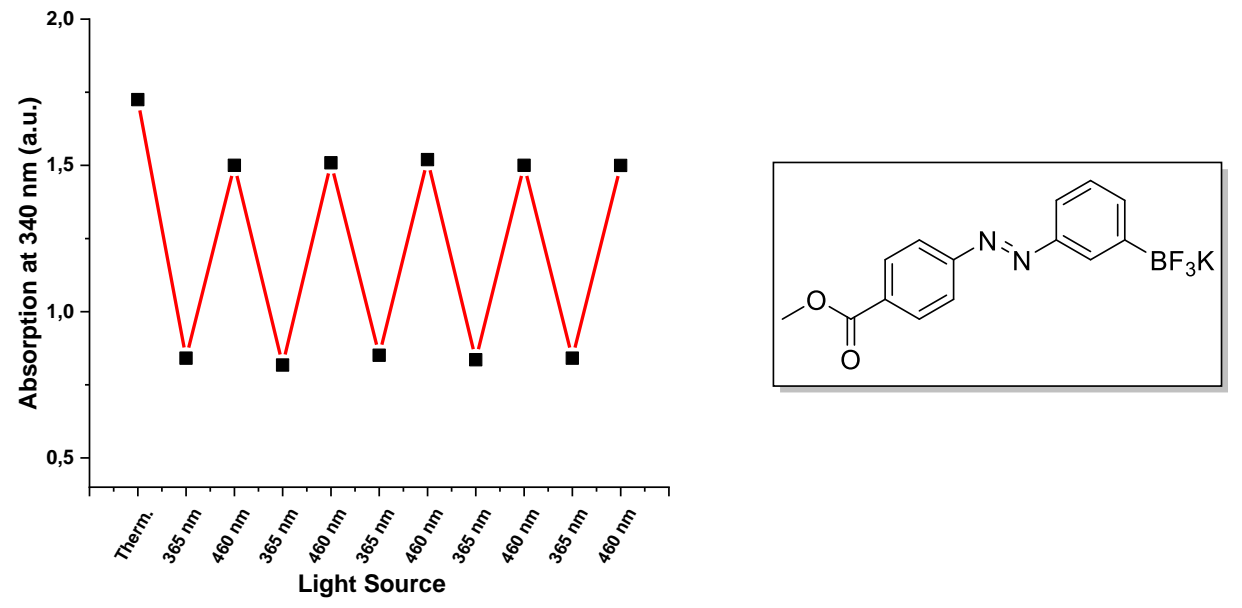

Figure S43. Photoreversibility of PI 15 in DMSO $(50 \mu \mathrm{M})$ at successive irradiation cycles of 365/460 nm.
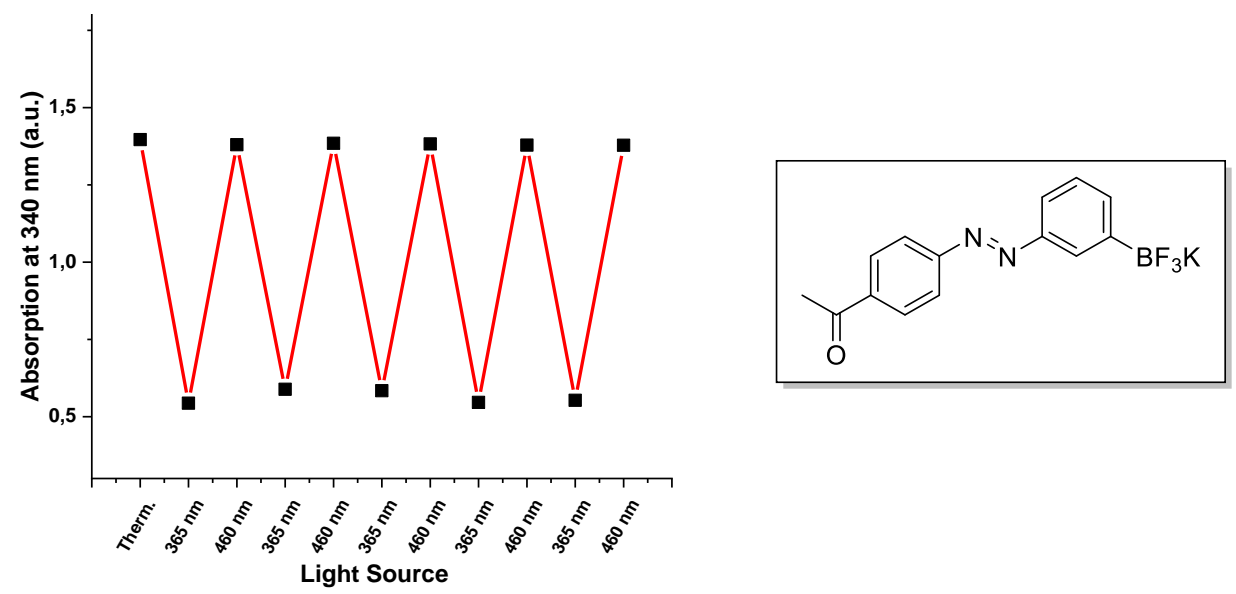

Figure S44. Photoreversibility of PI 16 in Tris/Ca ${ }^{2+}$ buffer $(50 \mu \mathrm{M})$ at successive irradiation cycles of $365 / 460 \mathrm{~nm}$.
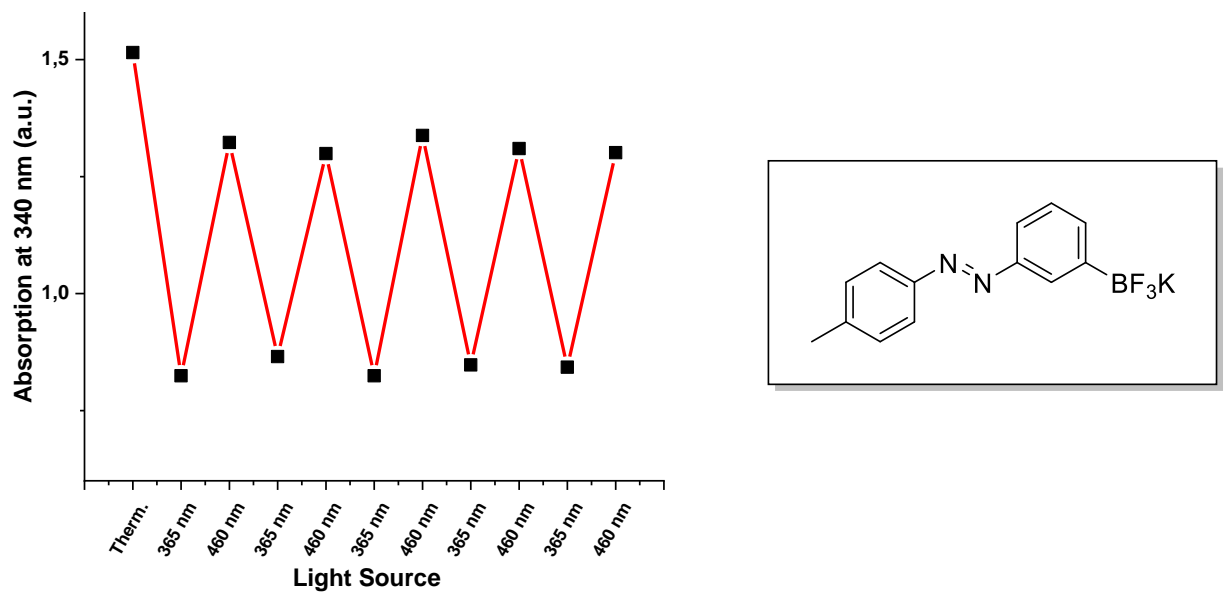

Figure S45. Photoreversibility of PI 17 in Tris/Ca ${ }^{2+}$ buffer $(50 \mu \mathrm{M})$ at successive irradiation cycles of 365/460 nm. 


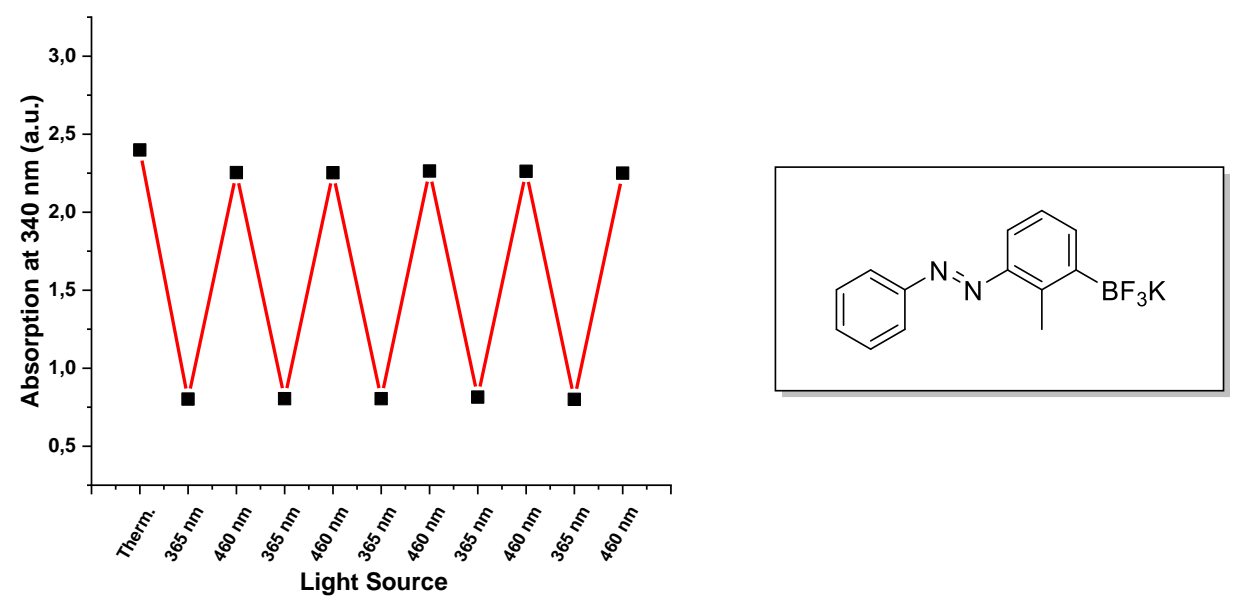

Figure S46. Photoreversibility of PI 18 in Tris/Ca ${ }^{2+}$ buffer $(50 \mu \mathrm{M})$ at successive irradiation cycles of $365 / 460 \mathrm{~nm}$.
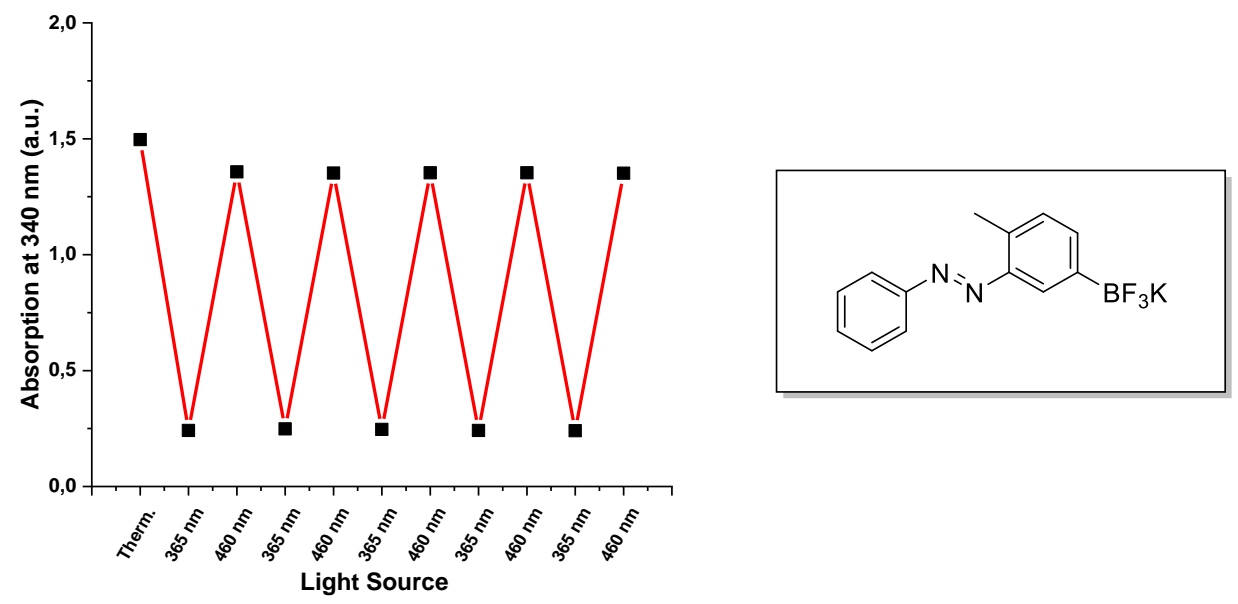

Figure S47. Photoreversibility of PI 19 in Tris/Ca ${ }^{2+}$ buffer $(50 \mu \mathrm{M})$ at successive irradiation cycles of $365 / 460 \mathrm{~nm}$.
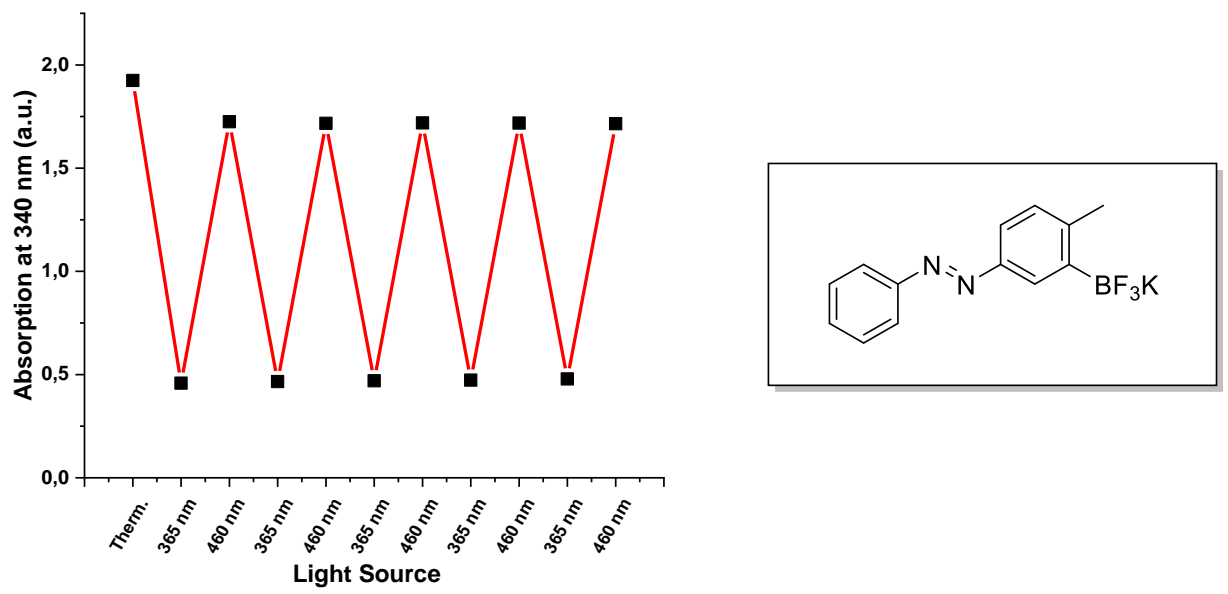

Figure S48. Photoreversibility of PI 20/PI in Tris/Ca ${ }^{2+}$ buffer $(50 \mu \mathrm{M})$ at successive irradiation cycles of $365 / 460 \mathrm{~nm}$. 

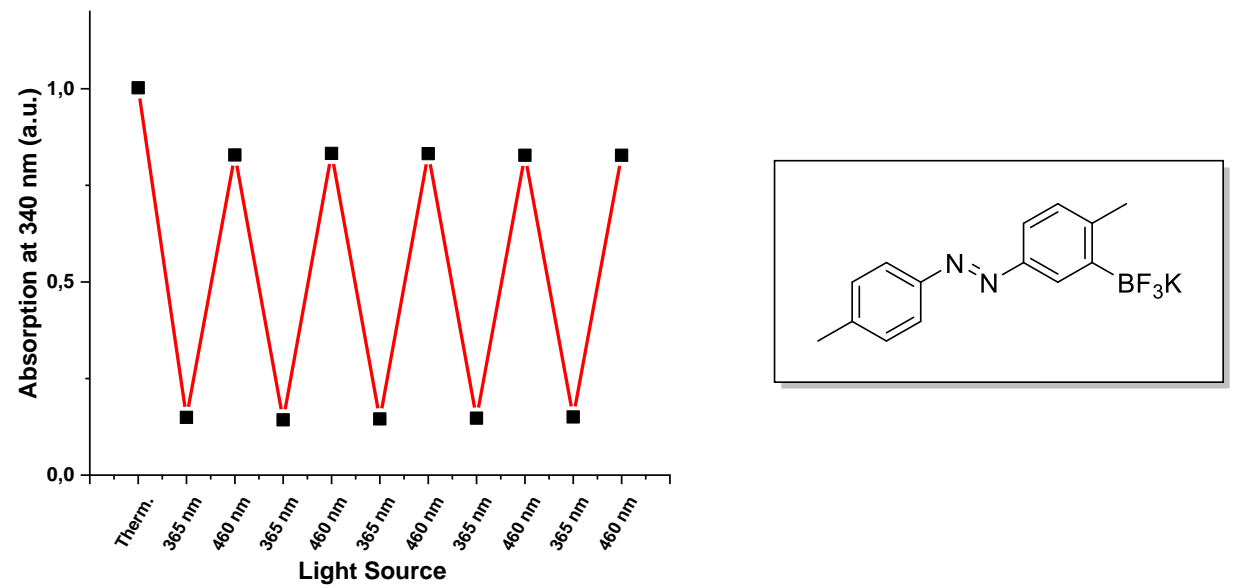

Figure S49. Photoreversibility of PI 21 in Tris/ $\mathrm{Ca}^{2+}$ buffer $(50 \mu \mathrm{M})$ at successive irradiation cycles of 365/460 nm. 


\subsection{Thermal Relaxation of cis-PIs}

\section{General Procedure and Results}

The irradiation of a thermally adapted (or with $460 \mathrm{~nm}$ irradiation regenerated) trans-configured PI with UV light $\left(\lambda_{\max }=365 \mathrm{~nm}\right.$ ) leads to an accumulation of the corresponding cis-configured PI due to trans $\rightarrow$ cis photoisomerization. For externally controlling enzymatic systems using PIs, the speed of the thermal cis $\rightarrow$ trans isomerization has to be investigated. A $50 \mu \mathrm{M}$ solution of the respective PI in a Tris/ $\mathrm{Ca}^{2+}(200 \mathrm{mM} / 20 \mathrm{mM}, \mathrm{pH}=7.8)$ buffer (or DMSO in case of limited water solubility of the compound) was irradiated for $5 \mathrm{~min}$ with UV light $\left(3 \mathrm{~W}, \lambda_{\max }=365 \mathrm{~nm}\right)$, ensuring that the photostationary state was reached. Then the solution was left at room temperature and recovery of the absorbance at the $\lambda_{\max }$ for the trans-isomer has been measured for $30 \mathrm{~min}$. Throughout all experiments, no significant thermal cis $\rightarrow$ trans isomerization was identified within the $30 \mathrm{~min}$ observation period. A slight increase of the $\lambda_{\max }$ for the $(E)$-isomer has been observed for 4, 14 and $\mathbf{1 5}$. However, due to the nature of the application of those PIs for the construction out-of-equilibrium ERN, the thermal cis $\rightarrow$ trans isomerization can also be neglected in those cases. The respective time-absorption spectra are shown below.
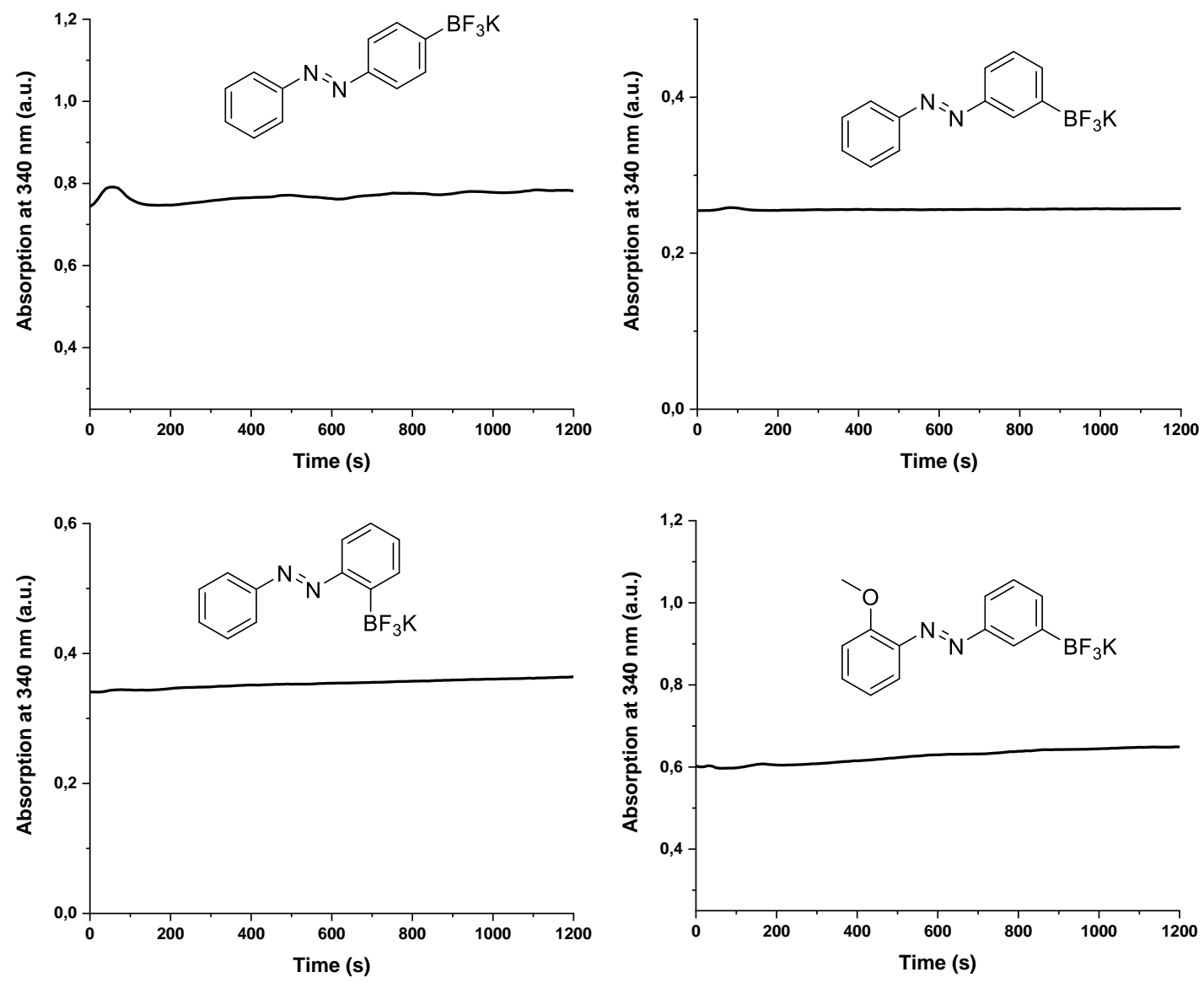

Figure S50. Time-Absorption spectra for the analysis of the thermal cis $\rightarrow$ trans isomerization of PIs. 

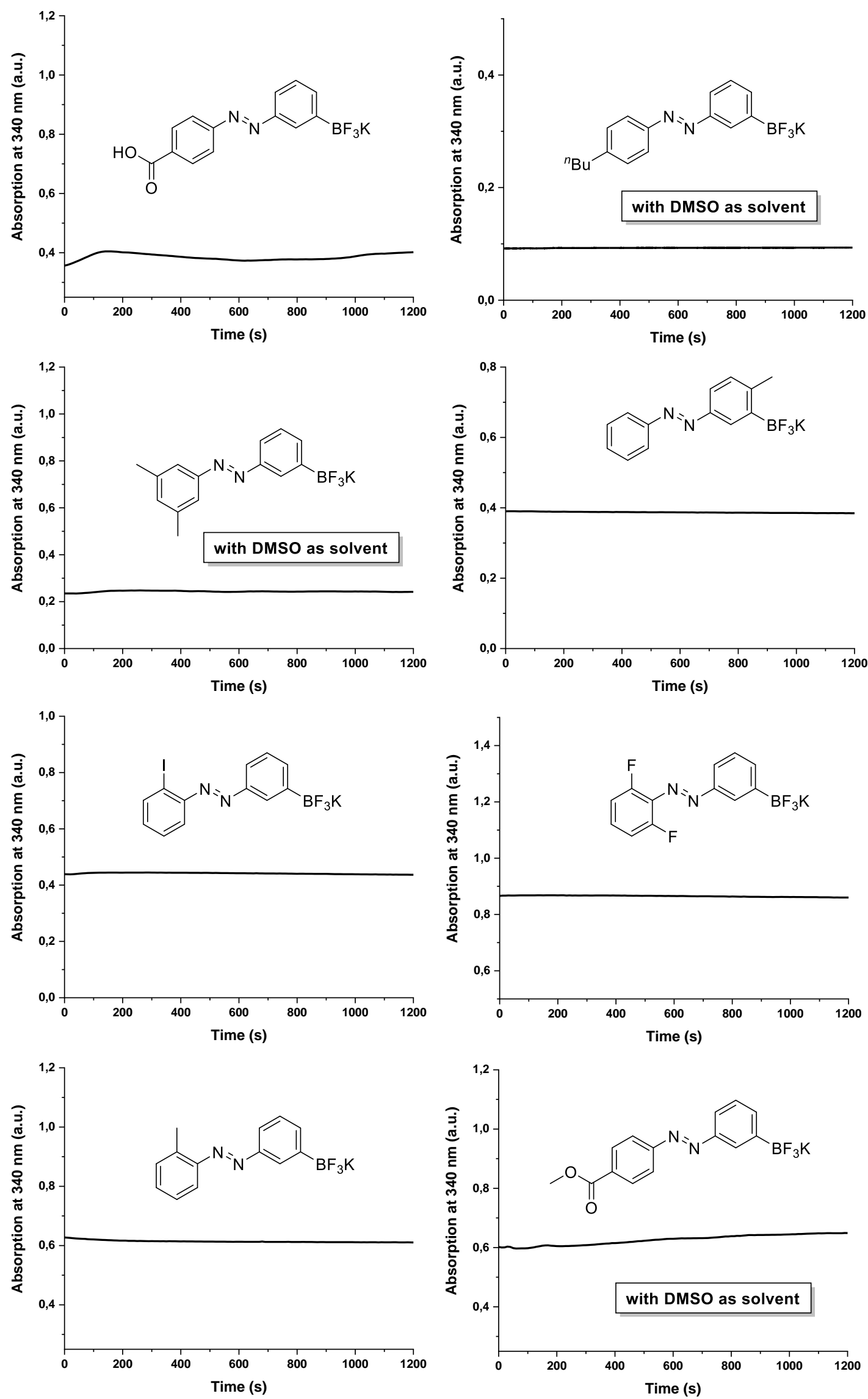

Figure S51. Time-Absorption spectra for the analysis of the thermal cis $\rightarrow$ trans isomerization of PIs. 

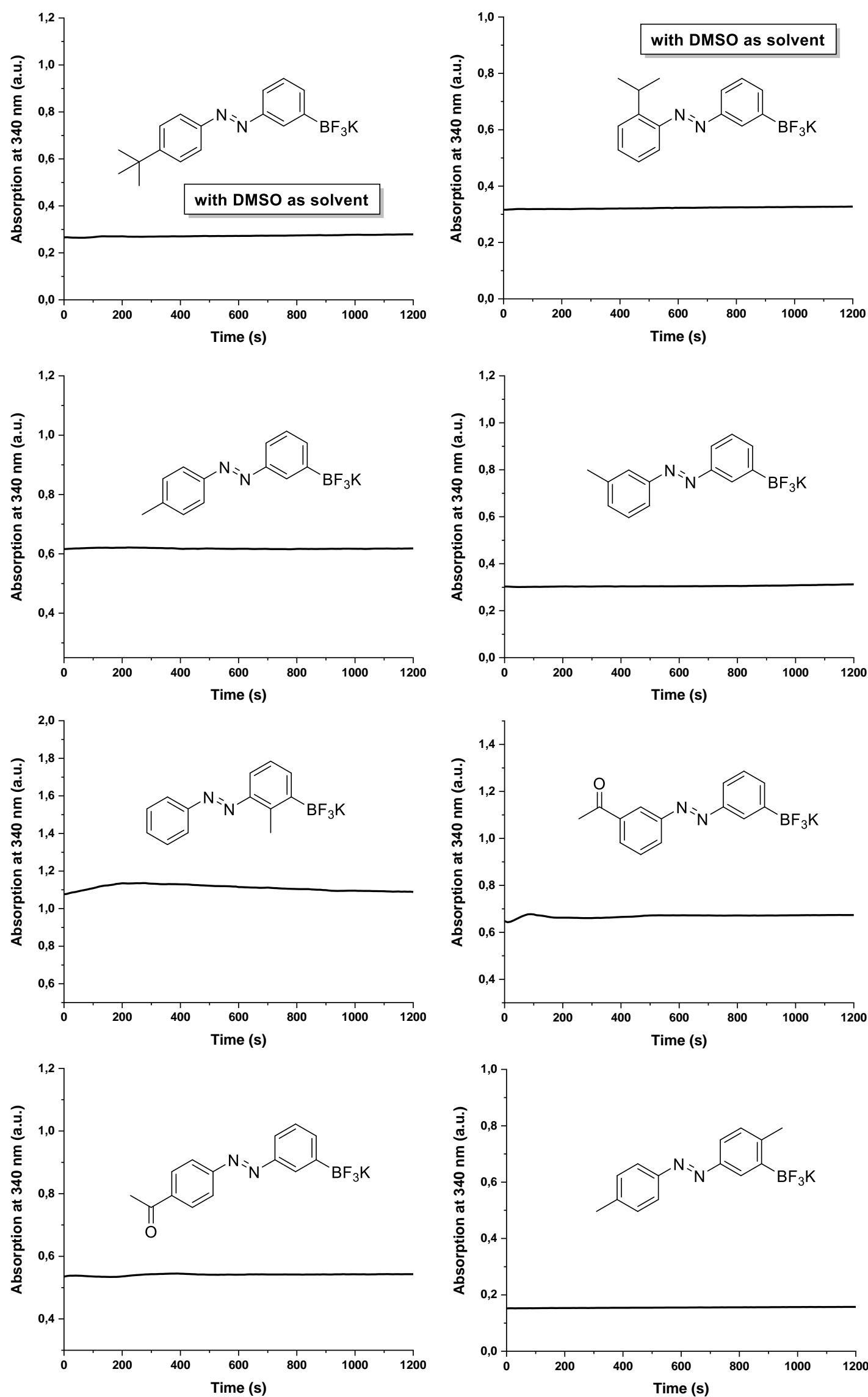

Figure S52. Time-Absorption spectra for the analysis of the thermal cis $\rightarrow$ trans isomerization of PIs. 

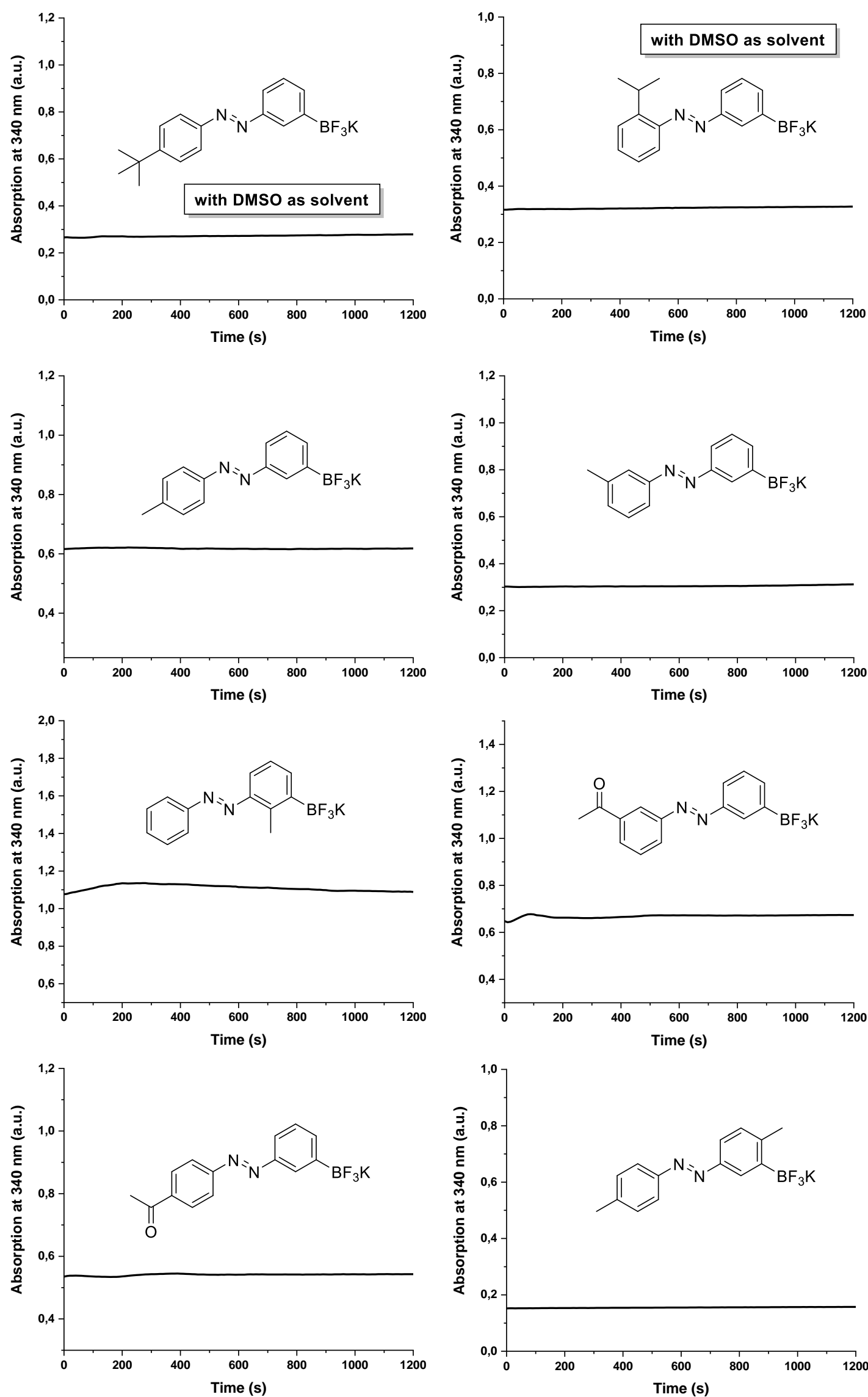

Figure S53. Time-Absorption spectra for the analysis of the thermal cis $\rightarrow$ trans isomerization of PIs. 


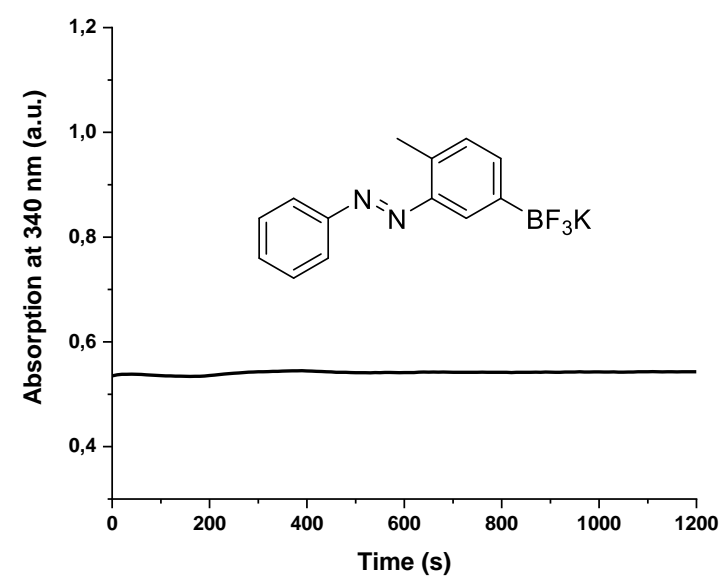

Figure S54. Time-Absorption spectra for the analysis of the thermal cis $\rightarrow$ trans isomerization of PIs.

\section{Determination of the thermal relaxation half-life of cis-20 (PI)}

A $35 \mu \mathrm{M}$ solution of PI (20) in a Tris/ $/ \mathrm{Ca}^{2+}(200 \mathrm{mM} / 20 \mathrm{mM}, \mathrm{pH}=7.8)$ buffer was irradiated for $5 \mathrm{~min}$ with UV light $\left(3 \mathrm{~W}, \lambda_{\max }=365 \mathrm{~nm}\right)$, ensuring that the photostationary state was reached. Then the solution was left at room temperature and recovery of the absorbance at the $\lambda_{\max }(330 \mathrm{~nm})$ for the transisomer has been measured using UV/Vis absorption spectroscopy for $12 \mathrm{~h}$ (see Figure S55). The obtained data was reversed (growth to decay), normalized (with respect to the maximum absorption of PI at its trans and cis state) and fitted with a single exponential decay function using Origin to determine the half-life of PI (20): $t_{1 / 2}=274 \mathrm{~h}$.
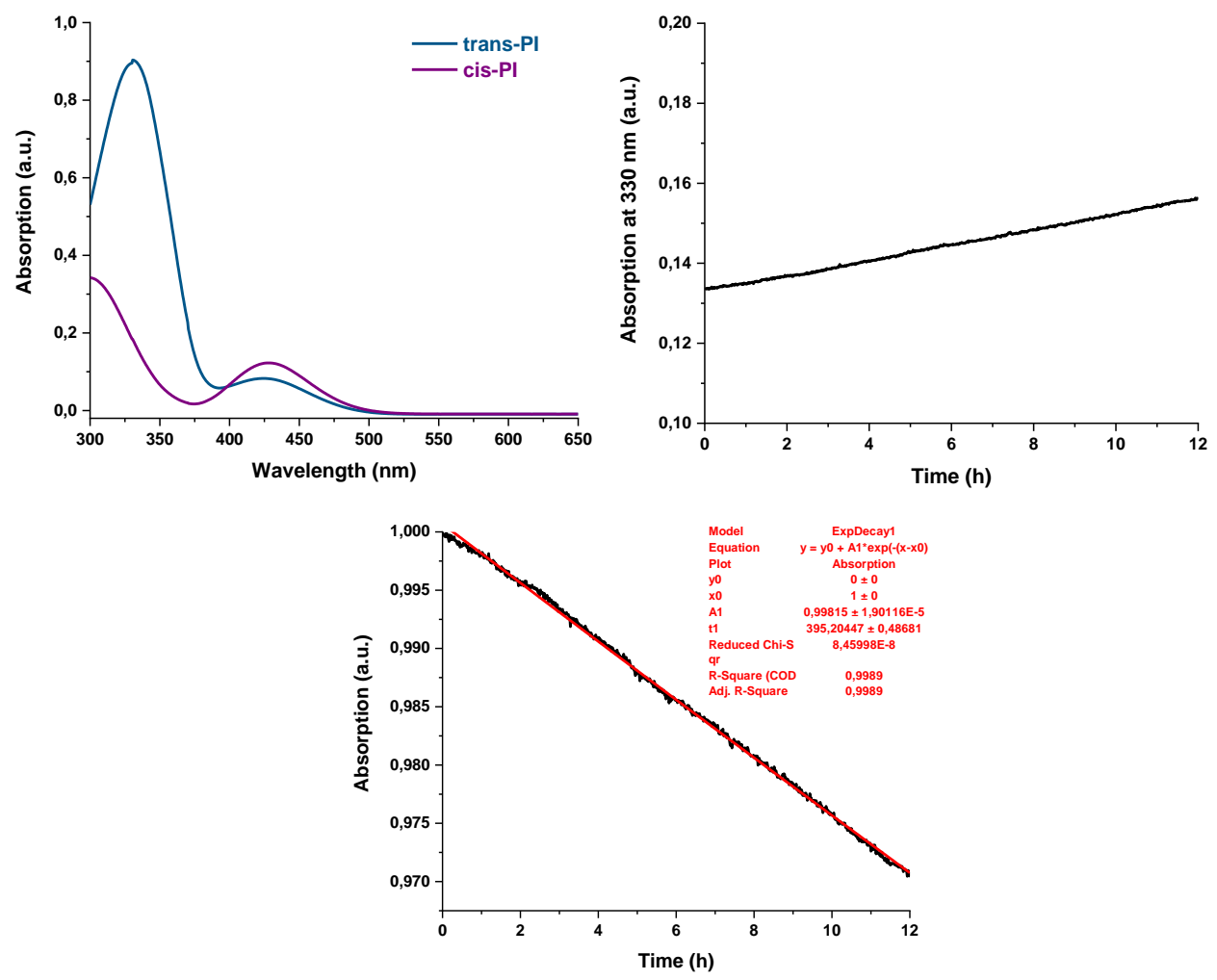

Figure S55. Determination of the thermal relaxation half-life of cis-20 (PI). 


\subsection{Determination of cis-PI Isomer Content at 365 nm PSS}

A solution of the respective PI $(\sim 2 \mathrm{mg} / \mathrm{mL})$ in DMSO- $d_{6}$ was irradiated with light from a UV-A LEDs ( $3 \mathrm{~W}, \lambda_{\max }=365 \mathrm{~nm}$ ) for $20 \mathrm{~min}$, thereby ensuring that the photostationary state has been reached. The cis-PI isomer content at $365 \mathrm{~nm}$ PSS was then determined via ${ }^{1} \mathrm{H}$ NMR analysis by comparing the characteristic proton signals for the cis- and the trans-isomer of the respective PI. The figures below show spectra of the starting (thermally-adapted, left) and of the $365 \mathrm{~nm}$ PSS (365 nm PSS, right) sample for all synthesized PIs. The results are summarized in Table S1.
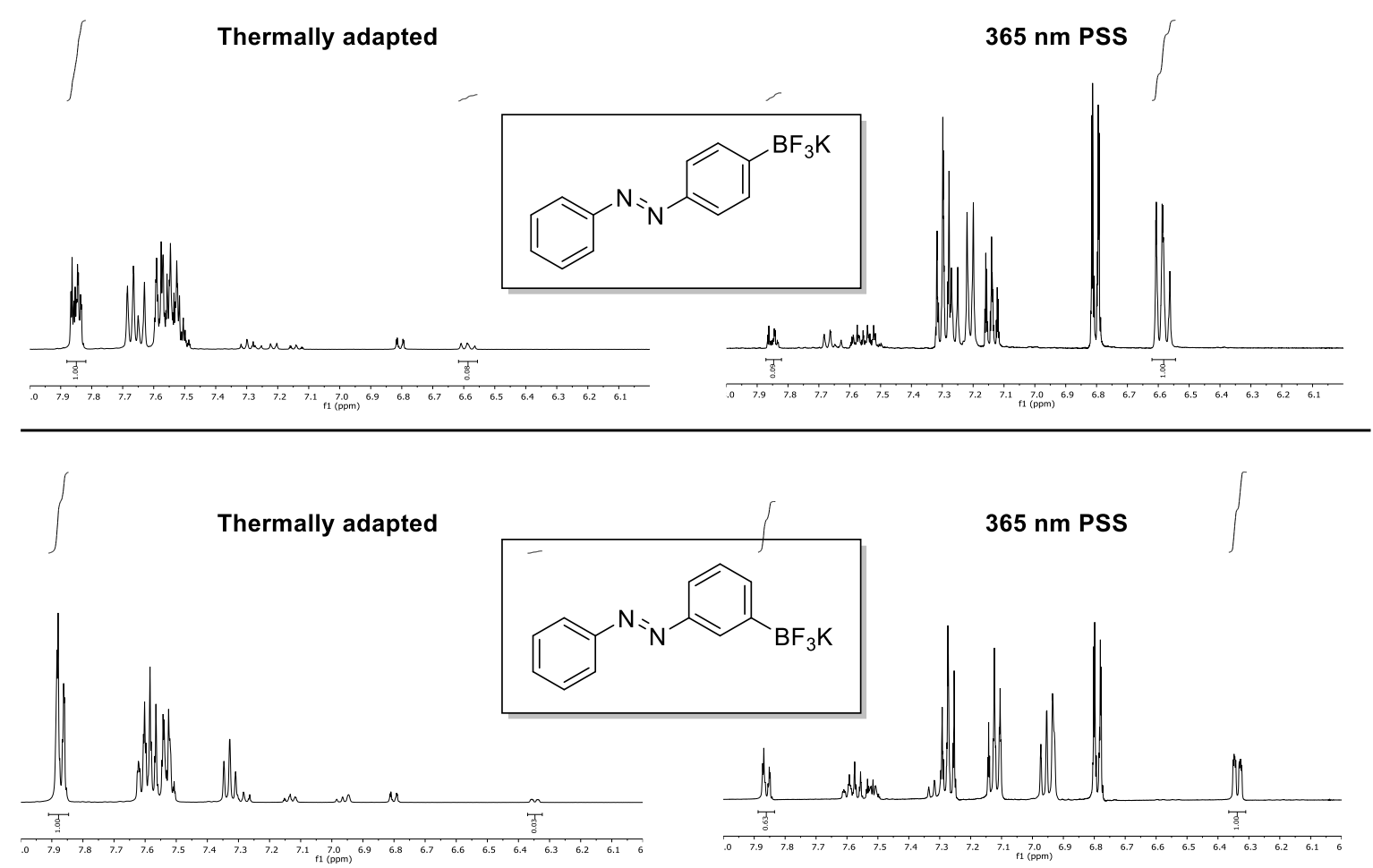

$365 \mathrm{~nm}$ PSS

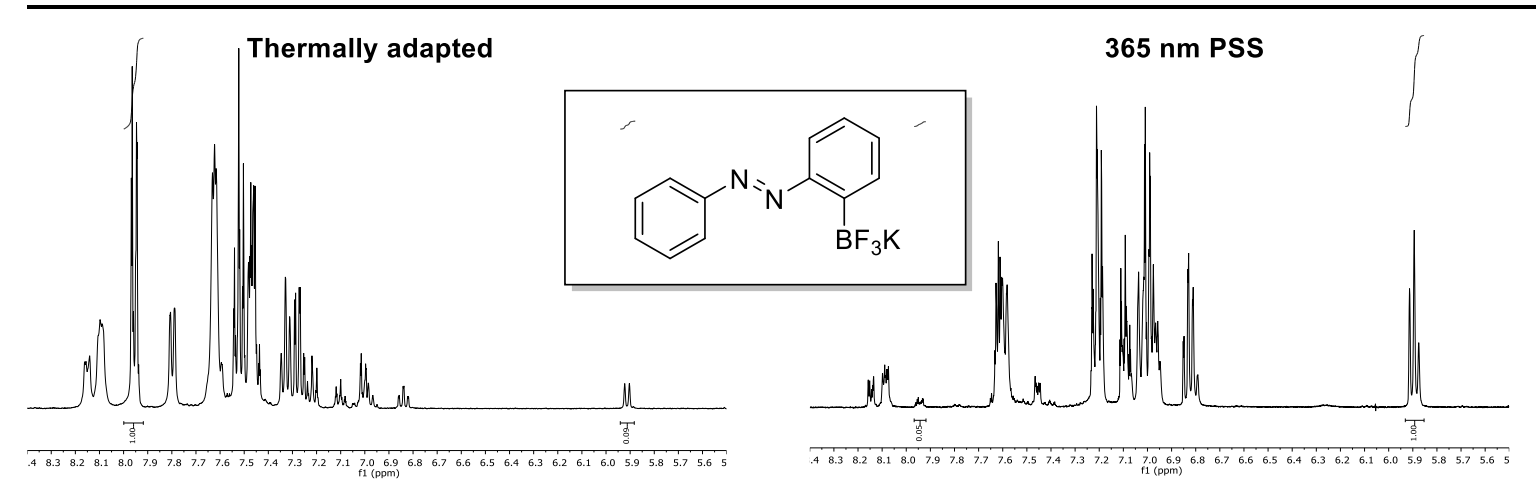

Figure S56. Analysis of $c i$ s-PI isomer content at $365 \mathrm{~nm}$ photostationary state and trans-PI isomer content at thermally adapted state by ${ }^{1} \mathrm{H}$ NMR spectroscopy. 

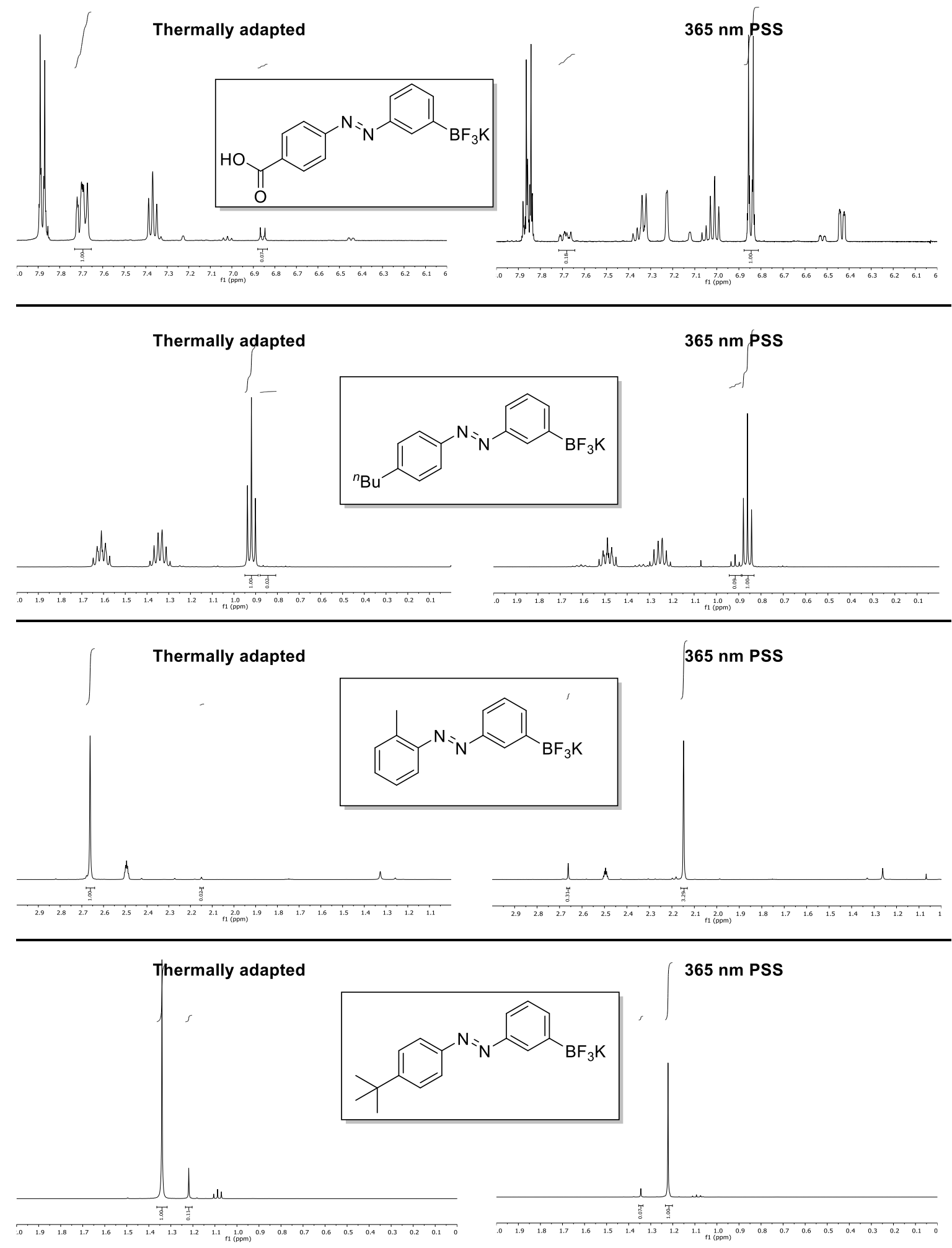

Figure S57. Analysis of cis-PI isomer content at $365 \mathrm{~nm}$ photostationary state and trans-PI isomer content at thermally adapted state by ${ }^{1} \mathrm{H}$ NMR spectroscopy. 


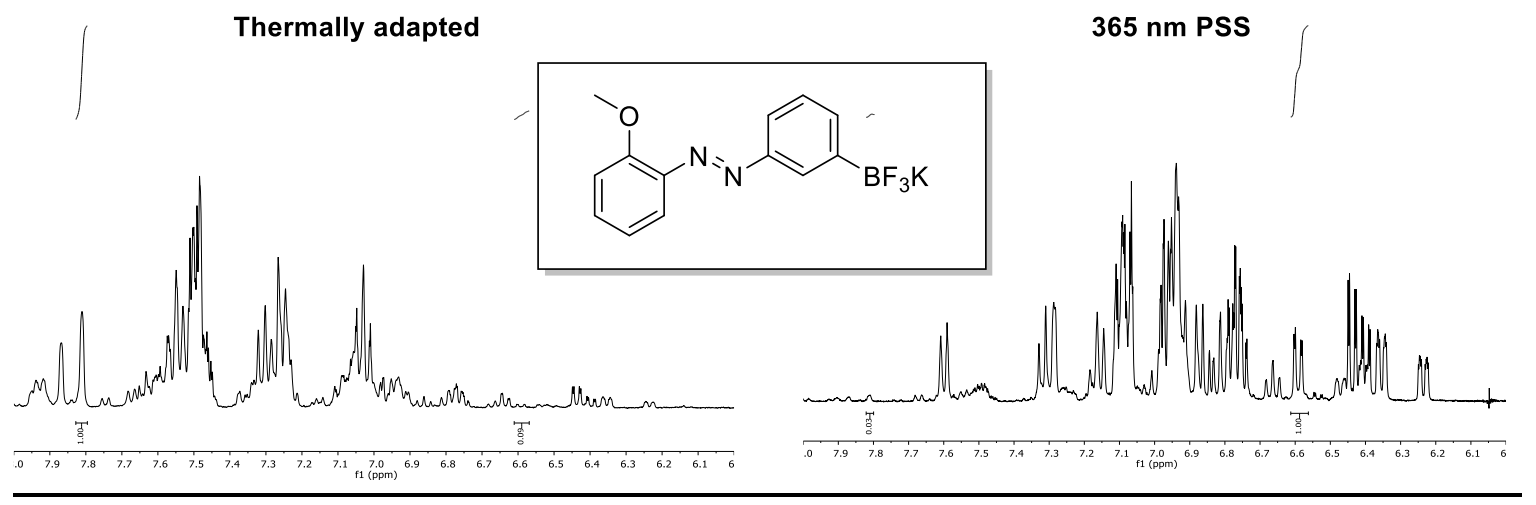

Thermally adapted

$365 \mathrm{~nm}$ PSS
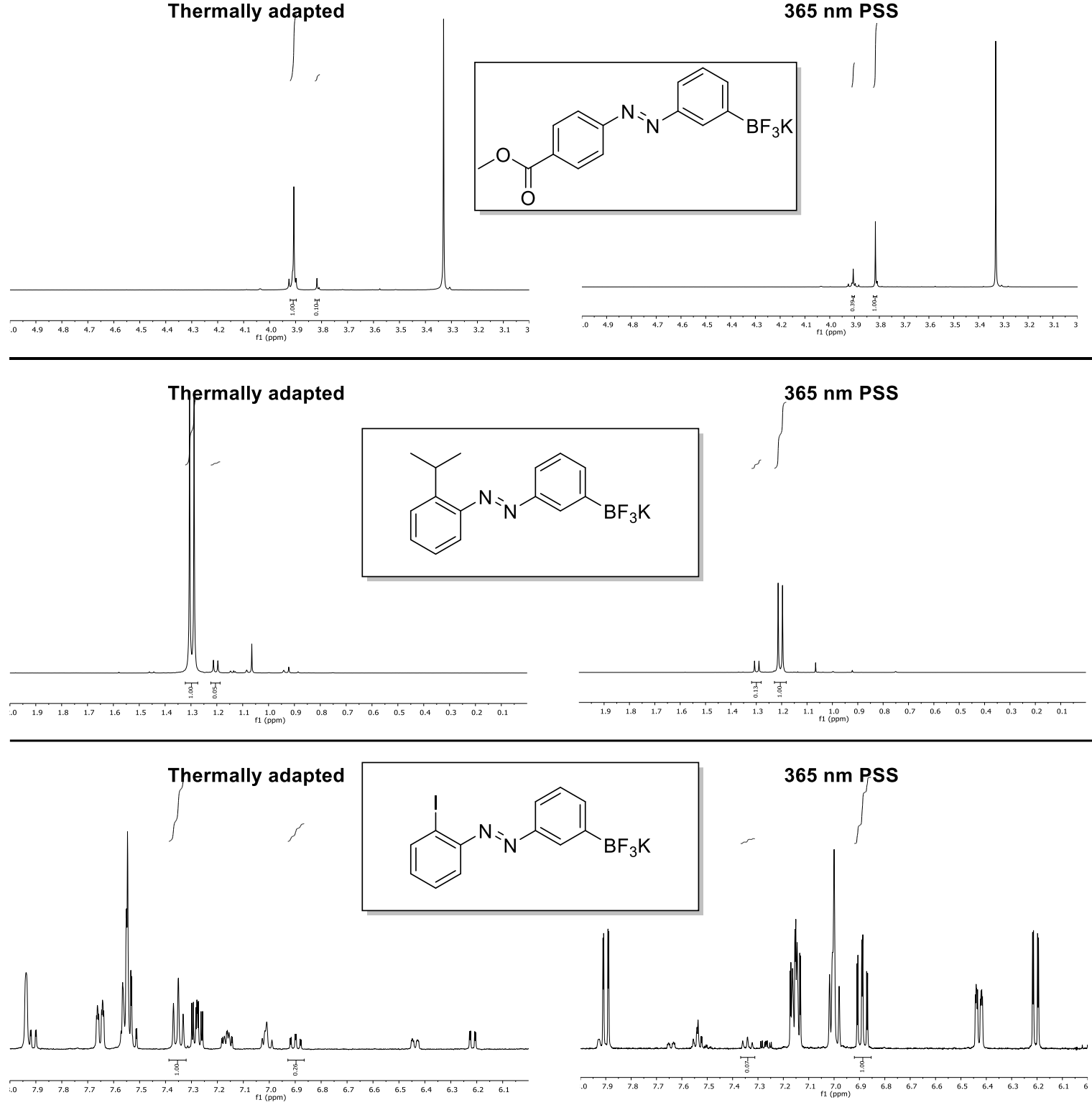

Figure S58. Analysis of cis-PI isomer content at $365 \mathrm{~nm}$ photostationary state and trans-PI isomer content at thermally adapted state by ${ }^{1} \mathrm{H}$ NMR spectroscopy. 

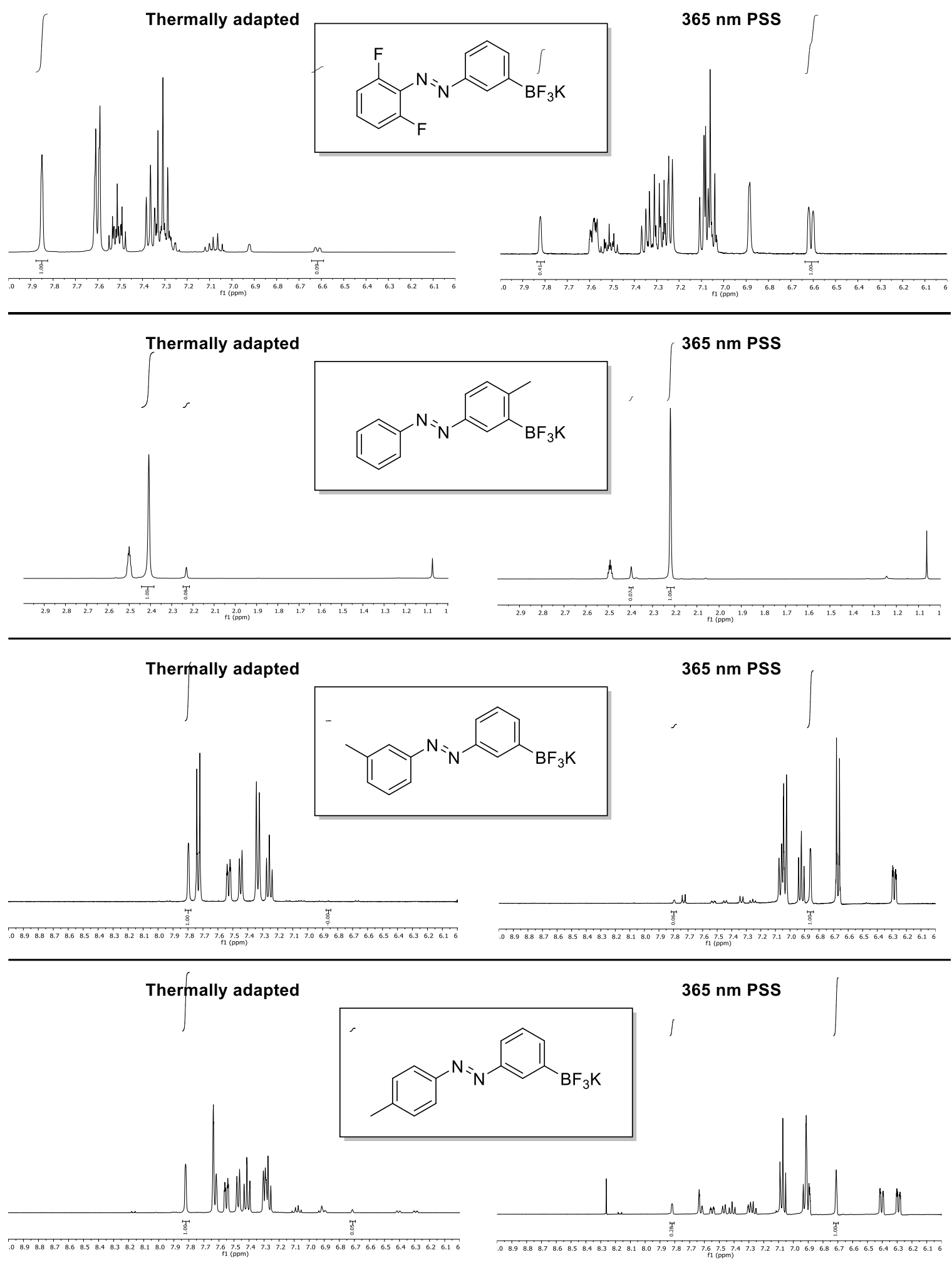

Figure S59. Analysis of cis-PI isomer content at $365 \mathrm{~nm}$ photostationary state and trans-PI isomer content at thermally adapted state by ${ }^{1} \mathrm{H}$ NMR spectroscopy. 

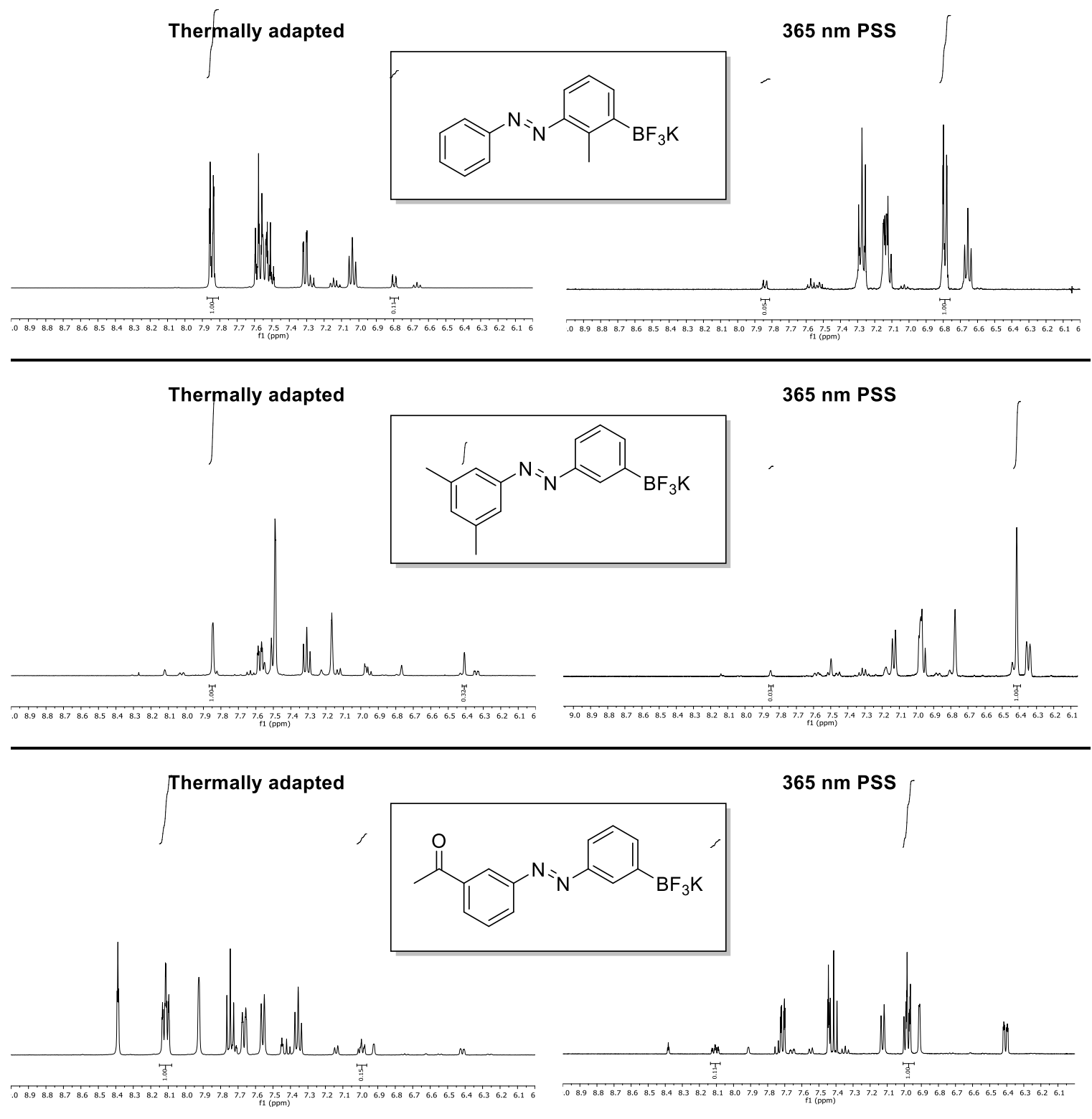

Thermally adapted

$365 \mathrm{~nm}$ PSS
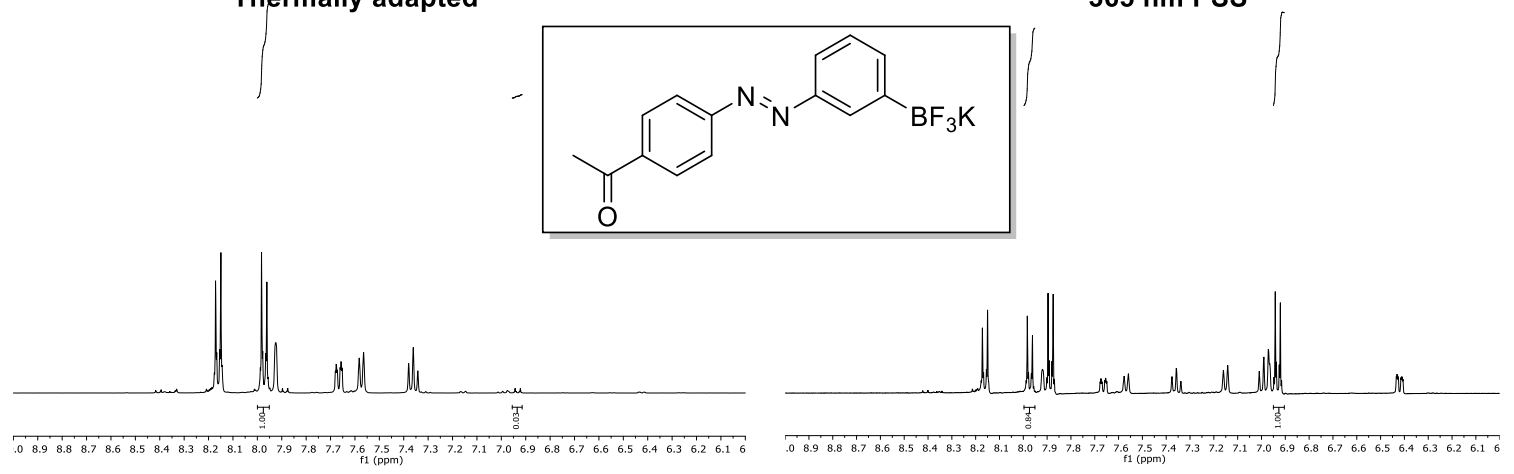

Figure S60. Analysis of $c i$ s-PI isomer content at $365 \mathrm{~nm}$ photostationary state and trans-PI isomer content at thermally adapted state by ${ }^{1} \mathrm{H}$ NMR spectroscopy. 

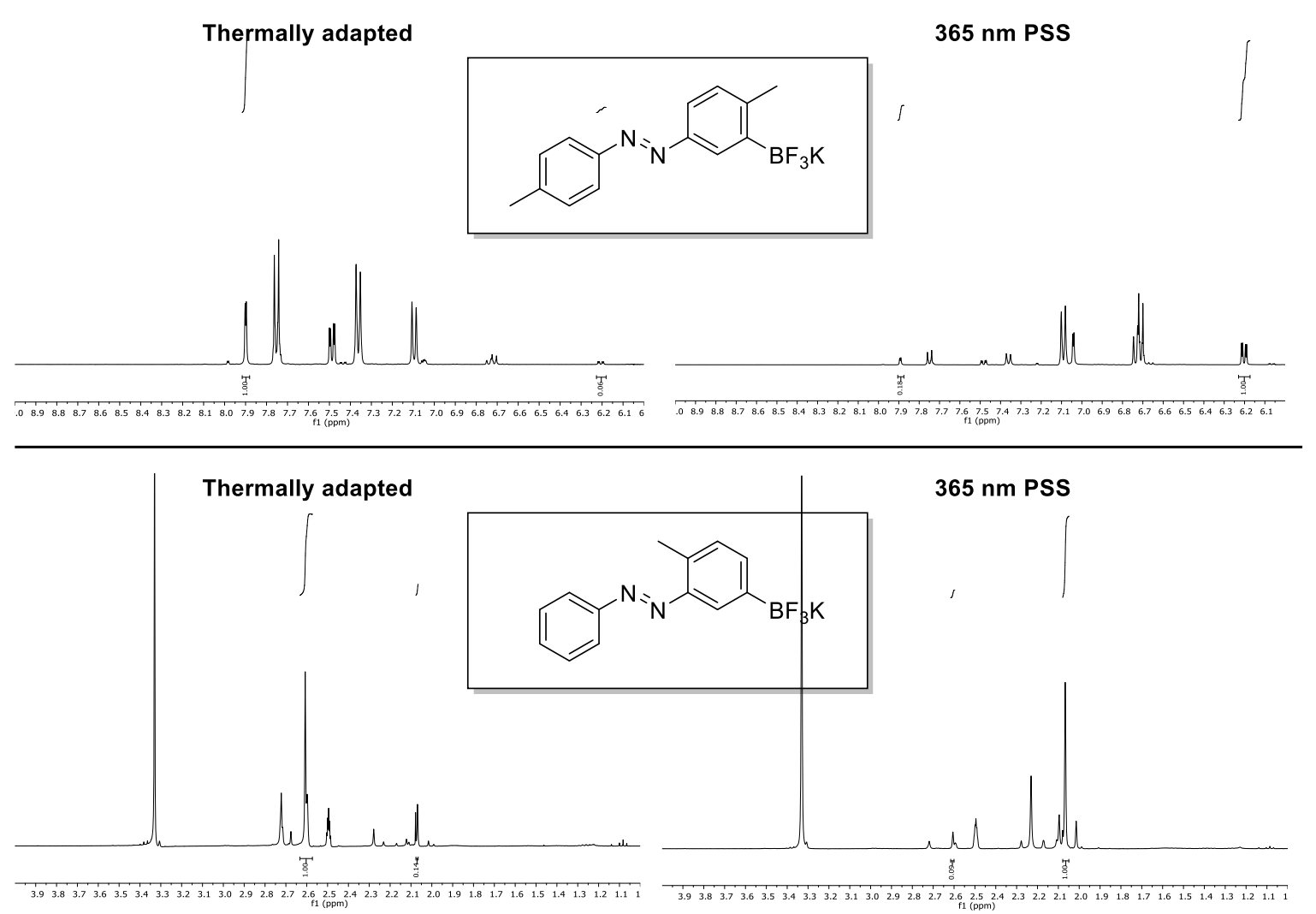

Figure S61. Analysis of cis-PI isomer content at $365 \mathrm{~nm}$ photostationary state and trans-PI isomer content at thermally adapted state by ${ }^{1} \mathrm{H}$ NMR spectroscopy. 
Table S1. Trans-PI isomer content of 1-21 at thermally adapted state and cis-PI isomer content at $365 \mathrm{~nm}$ photostationary state determined by ${ }^{1} \mathrm{H}$ NMR spectroscopy.

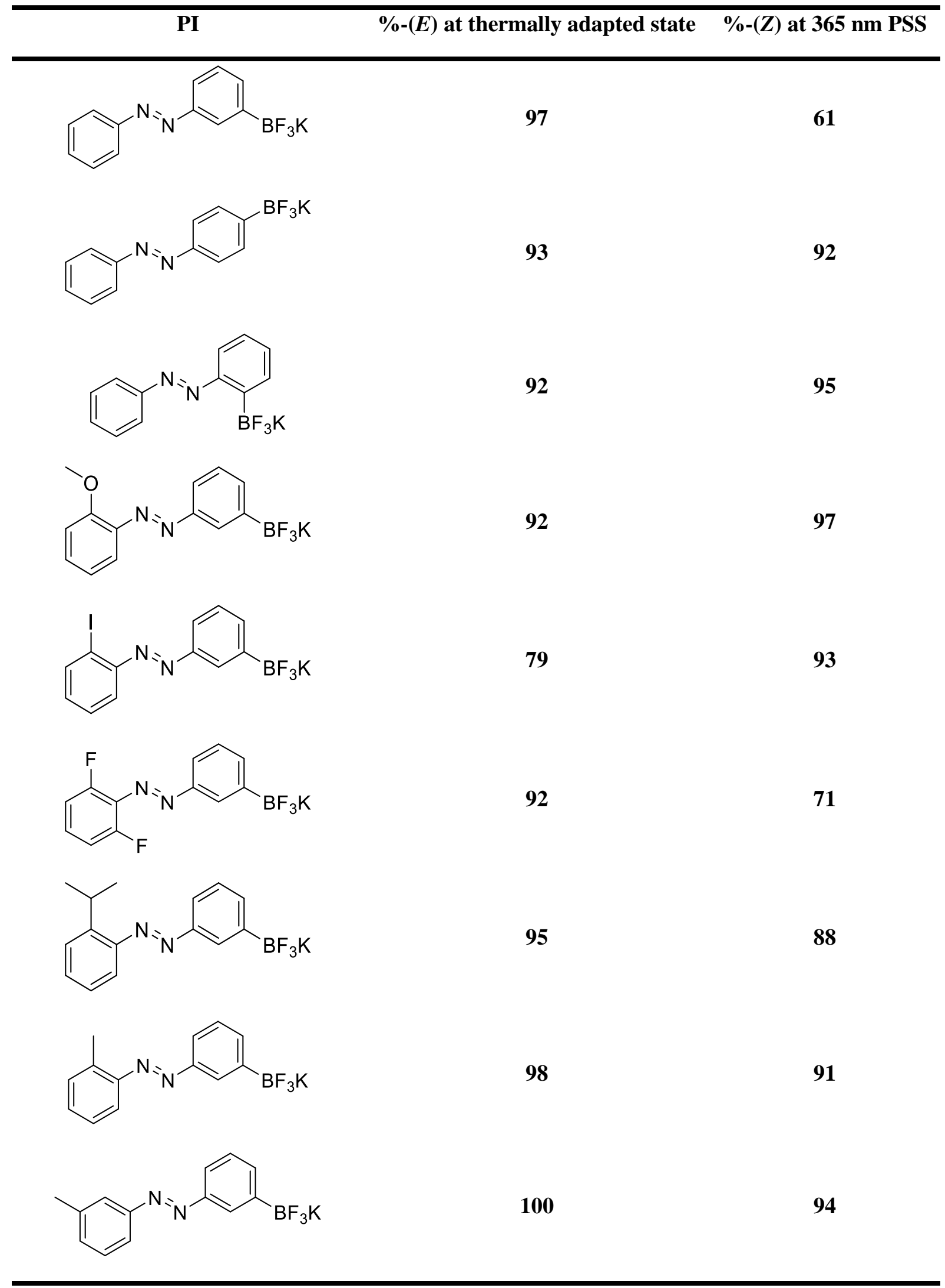




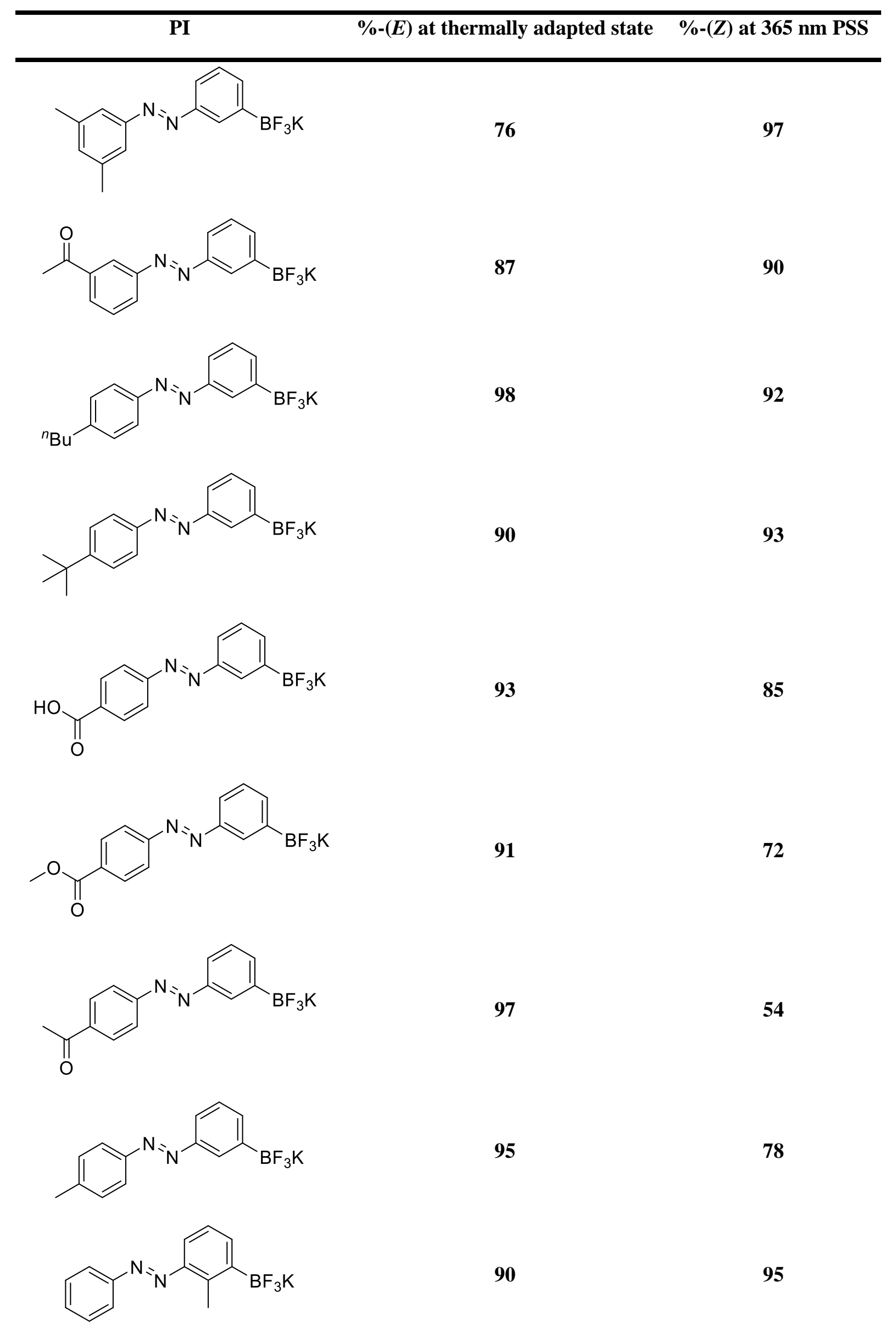




\%-(E) at thermally adapted state \%-(Z) at 365 nm PSS




\section{Enzymological Characterization of PIs}

\subsection{Preparation of Stock Solutions}

All required stock solutions were prepared as outlined below:

Tris/Ca ${ }^{2+} / \mathrm{BSA}$ buffer $(200 \mathrm{mM} / 20 \mathrm{mM} / 0.1 \mathrm{wt} \%$; $\mathrm{pH}=\mathbf{7 . 8 1})$ - no DMSO (Buffer)

The Tris/ $\mathrm{Ca}^{2+}$ buffer $(200 \mathrm{mM} / 20 \mathrm{mM}$; $\mathrm{pH}=7.81)$ was prepared by adding $50 \mathrm{~mL}$ of a $1 \mathrm{M}$ Tris solution and $2.5 \mathrm{~mL}$ of a $2 \mathrm{M} \mathrm{CaCl}_{2}$ solution to $197.5 \mathrm{~mL}$ of MilliQ. The $\mathrm{pH}$ was adjusted by the dropwise addition of $1 \mathrm{M} \mathrm{HCl}$. By adding $50 \mathrm{mg}$ of BSA to $50 \mathrm{~mL}$ of the Tris/ $\mathrm{Ca}^{2+}$ buffer $(200 \mathrm{mM} / 20 \mathrm{mM}$; $\mathrm{pH}=7.81)$, the Tris/ $\mathrm{Ca}^{2+} / \mathrm{BSA}$ buffer $(200 \mathrm{mM} / 20 \mathrm{mM} / 0.1 \mathrm{wt} \% ; \mathrm{pH}=7.81)$ was obtained. This buffer was prepared freshly for each experiment/set of experiments due to the potential decomposition of BSA over time (BSA is added to prevent the adsorption of $\mathrm{Cr}$ onto plastic, e.g. pipette tips or tubing).

Tris/Ca ${ }^{2+} / \mathrm{BSA}$ buffer $(200 \mathrm{mM} / 20 \mathrm{mM} / 0.1 \mathrm{wt} \% ; \mathrm{pH}=7.81)-2$ vol\% DMSO (Buffer-DMSO) The Tris/ $\mathrm{Ca}^{2+}$ buffer $(200 \mathrm{mM} / 20 \mathrm{mM} ; \mathrm{pH}=7.81)$ was prepared by adding $50 \mathrm{~mL}$ of a $1 \mathrm{M}$ Tris solution and $2.5 \mathrm{~mL}$ of a $2 \mathrm{M} \mathrm{CaCl}_{2}$ solution to $197.5 \mathrm{~mL}$ of MilliQ. The $\mathrm{pH}$ was adjusted by the dropwise addition of $1 \mathrm{M} \mathrm{HCl}$. By adding $50 \mathrm{mg}$ of BSA to $49 \mathrm{~mL}$ of the Tris/ $\mathrm{Ca}^{2+}$ buffer $(200 \mathrm{mM} / 20 \mathrm{mM}$; $\mathrm{pH}=7.81$ ) followed by the addition of $1 \mathrm{~mL}$ DMSO to this solution, the Tris $/ \mathrm{Ca}^{2+} / \mathrm{BSA}^{\mathrm{B}}$ buffer $(200 \mathrm{mM} / 20 \mathrm{mM} / 0.1 \mathrm{wt} \%$; $\mathrm{pH}=7.81)$ containing 2 vol\% DMSO was obtained. This buffer was prepared freshly for each experiment/set of experiments due to the potential decomposition of BSA over time. To prevent thermal degradation of BSA upon DMSO addition, the latter solvent should be added slowly while stirring the solution.

\section{Suc-Ala-Ala-Pro-Phe-AMC (400 $\boldsymbol{\mu M})$ Cr substrate solution (Cr-substrate)}

$26.6 \mu \mathrm{L}$ of a Suc-Ala-Ala-Pro-Phe-AMC stock solution (150 mM in DMSO) were diluted with 9973.3 $\mu \mathrm{L}$ of Buffer or Buffer-DMSO to obtain $10 \mathrm{~mL}$ of a $400 \mu \mathrm{M}$ Suc-Ala-Ala-Pro-Phe-AMC Cr substrate solution. The Cr-substrate solution was prepared freshly for each experiment/set of experiments due to the potential decomposition of the fluorophore and BSA over time.

\section{Chymotrypsin enzyme solution ( $0.2 \mu \mathrm{M}$, Cr-enzyme)}

$200 \mu \mathrm{L}$ of chymotrypsin $\left(10 \mu \mathrm{M}\right.$ in $\left.2 \mathrm{mM} \mathrm{HCl} / 20 \mathrm{mM} \mathrm{Ca}^{2+}\right)$ were diluted with $9800 \mu \mathrm{L}$ of Buffer or Buffer-DMSO. The enzyme solution was prepared freshly for each experiment/set of experiments due to the decomposition of chymotrypsin or BSA over time.

\section{Chymotrypsin photoswitchable inhibitor solution (1 mM, PI)}

$4 \mu \mathrm{mol}$ of the respective PI were dissolved in $4 \mathrm{~mL}$ of Buffer or Buffer-DMSO, depending on the solubility of the compound.

\subsection{AMC Calibration}

To correlate the fluorescence signal, produced via enzymatic cleavage of the peptide-AMC substrate, to an AMC concentration, an external calibration using AMC (10 $\mu \mathrm{M}$ in DMSO) was performed. The fluorescence intensity of wells within 96-well plates (purchased from Greiner Bio-One), containing $200 \mu \mathrm{L}$ of the respective calibration solution, was monitored for $10 \mathrm{~s}$ (shaking 3s/orbital mode/amplitude $4 \mathrm{~mm})$ at $25^{\circ} \mathrm{C}$ using top fluorescence reading mode $\left(\lambda_{\mathrm{ex}} / \lambda_{\mathrm{em}}=380 \mathrm{~nm} / 460 \mathrm{~nm}\right)$, as described in the 
General Information (see 1.1 of supplementary information). The calibration curve and the dilution scheme are depicted in Fig. S62.

\begin{tabular}{cccc}
\hline$[\mathrm{AMC}] / \boldsymbol{\mu M}$ & $\mathrm{V}(\mathbf{1 0} \boldsymbol{\mu M}$ AMC stock) $/ \boldsymbol{\mu L}$ & $\mathrm{V}$ (Buffer) $/ \boldsymbol{\mu L}$ & Total $\mathbf{L} / \boldsymbol{\mu L}$ \\
\hline 0 & 0 & 200 & 200 \\
3 & 60 & 140 & 200 \\
4 & 80 & 120 & 200 \\
5 & 100 & 100 & 200 \\
6 & 120 & 80 & 200 \\
7 & 140 & 60 & 200 \\
8 & 160 & 40 & 200 \\
10 & 200 & 0 & 200 \\
\hline
\end{tabular}

$[\mathrm{AMC}] / \mu \mathrm{M}=(0.00187 \pm 0.00002)(\mu \mathrm{M} / \mathrm{a} . \mathrm{u}) *$ Fluorescence

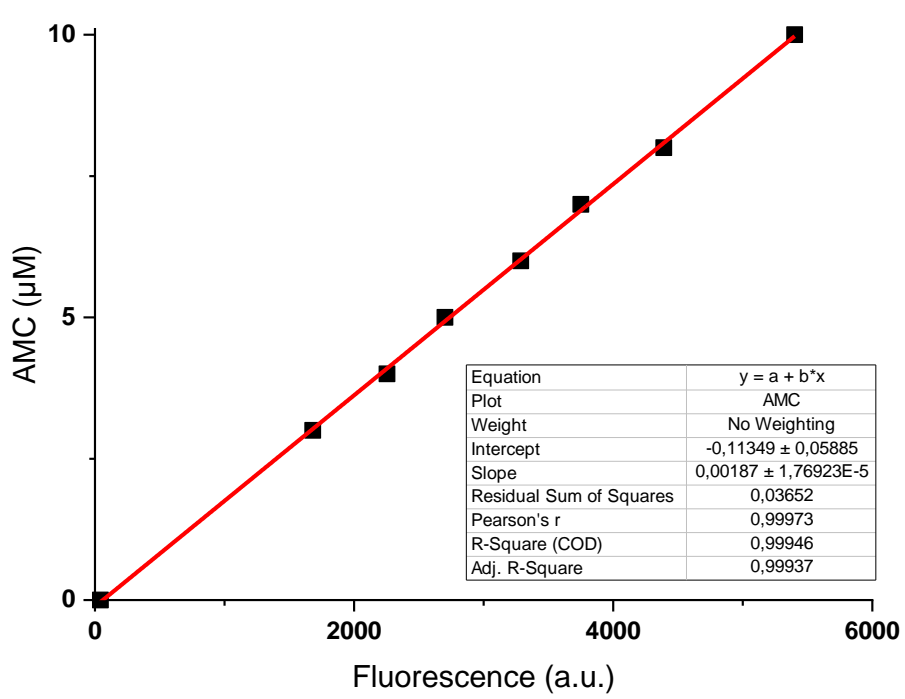

Figure S62. Calibration curve correlating values of fluorescence intensity to concentration of AMC. 


\subsection{Michaelis-Menten Analysis of Cr/AAPF-AMC System}

The determination of the inhibition constant $\left(K_{\mathrm{i}}\right)$ of the synthesized PIs requires the knowledge of the maximum reaction velocity $\left(V_{\max }\right)$, the Michaelis constant $\left(K_{\mathrm{M}}\right)$ and the turnover number $\left(k_{\mathrm{cat}}\right)$ of the chymotrypsin/Suc-AAPF-AMC fluorogenic assay. Fluorogenic assays were performed at fixed enzyme concentration with varied substrate concentration as outlined in Table S2.

Table S2. Pipetting scheme for the Michaelis-Menten analysis of the Cr/AAPF-AMC system. All experiments were done as triplicates using the autoinjector of the Tecan SparkM10 plate reader for the addition of the Cr-enzyme to each well prior to the respective measurement. Buffer-DMSO was used instead of Buffer in case of low water solubility of the PI.

\begin{tabular}{cccccc}
\hline $\begin{array}{c}{[\text { Cr-substrate }]_{\text {well }} /} \\
\boldsymbol{\mu M}\end{array}$ & $\begin{array}{c}\mathbf{V}(\mathbf{C r} \text {-substrate, } \mathbf{4 0 0} \\
\boldsymbol{\mu M} \text { stock }) / \boldsymbol{\mu L}\end{array}$ & $\begin{array}{c}{[\text { Cr-enzyme }]_{\text {well }} /} \\
\boldsymbol{\mu M}\end{array}$ & $\begin{array}{c}\mathbf{V}(\mathbf{C r} \text {-enzyme, } \mathbf{0 . 4} \\
\boldsymbol{\mu M} \text { stock }) / \boldsymbol{\mu} \mathbf{L}\end{array}$ & $\begin{array}{c}\text { Buffer or Buffer- } \\
\mathbf{D M S O} / \boldsymbol{\mu L}\end{array}$ & Total Volume $/ \boldsymbol{\mu L}$ \\
\hline 10 & 5 & 0.02 & 10 & 185 & 200 \\
20 & 10 & 0.02 & 10 & 180 & 200 \\
30 & 15 & 0.02 & 10 & 175 & 200 \\
40 & 20 & 0.02 & 10 & 170 & 200 \\
60 & 30 & 0.02 & 10 & 160 & 200 \\
80 & 40 & 0.02 & 10 & 150 & 200 \\
100 & 50 & 0.02 & 10 & 140 & 200 \\
120 & 60 & 0.02 & 10 & 130 & \\
\hline
\end{tabular}

All compounds except chymotrypsin have been pipetted using multichannel pipettes. The enzyme chymotrypsin was then added to the respective well of the 96 well-plate using the Tecan SparkM10 autoinjector. All experiments were done as triplicates. The fluorescence intensity of wells within 96well plates (purchased from Greiner Bio-One), containing $200 \mu \mathrm{L}$ of the respective solution, was monitored for $40 \mathrm{~s}$ (shaking 3s/orbital mode/amplitude $4 \mathrm{~mm}$ ) at $25^{\circ} \mathrm{C}$ using top fluorescence reading mode $\left(\lambda_{\text {ex }} / \lambda_{\mathrm{em}}=380 \mathrm{~nm} / 460 \mathrm{~nm}\right)$, as described in the General Information (see 1.1) part of this supplementary information. The amount of produced fluorescent product was calculated according to the AMC calibration curve (4.2). The data was fit with the Michaelis-Menten equation (Eq. 1).

$$
v=\frac{V_{\max }[S]}{K_{M}+[S]}
$$

This plot predicts reaction velocity as a function of concentration and allows quantification of $k_{\text {cat }}$ and $K_{\mathrm{M}}$. Values of $k_{\text {cat }}$ were calculated according to Eq. 2.

$$
k_{c a t}=\frac{V_{\max }}{[E]}
$$

The maximum reaction velocity $\left(V_{\max }\right)$, the Michaelis constant $\left(K_{\mathrm{M}}\right)$ and the turnover number $\left(k_{\mathrm{cat}}\right)$ of the chymotrypsin/Suc-AAPF-AMC fluorogenic assay were then obtained from fitting a MichaelisMenten-type to the experimental data using the Origin 2018b software package. The Michaelis-Menten analysis of the chymotrypsin/Suc-AAPF-AMC fluorogenic system was performed for each experiment/set of experiments to take dilution and weighing errors, differences in activity of the respective enzyme batch and other errors into account. An exemplary Michaelis-Menten plot of the chymotrypsin/Suc-AAPF-AMC fluorogenic assay and the determined maximum reaction velocity $\left(V_{\max }\right)$, the Michaelis constant $\left(K_{\mathrm{M}}\right)$ and the turnover number $\left(k_{\mathrm{cat}}\right)$ are depicted in Figure S63. 


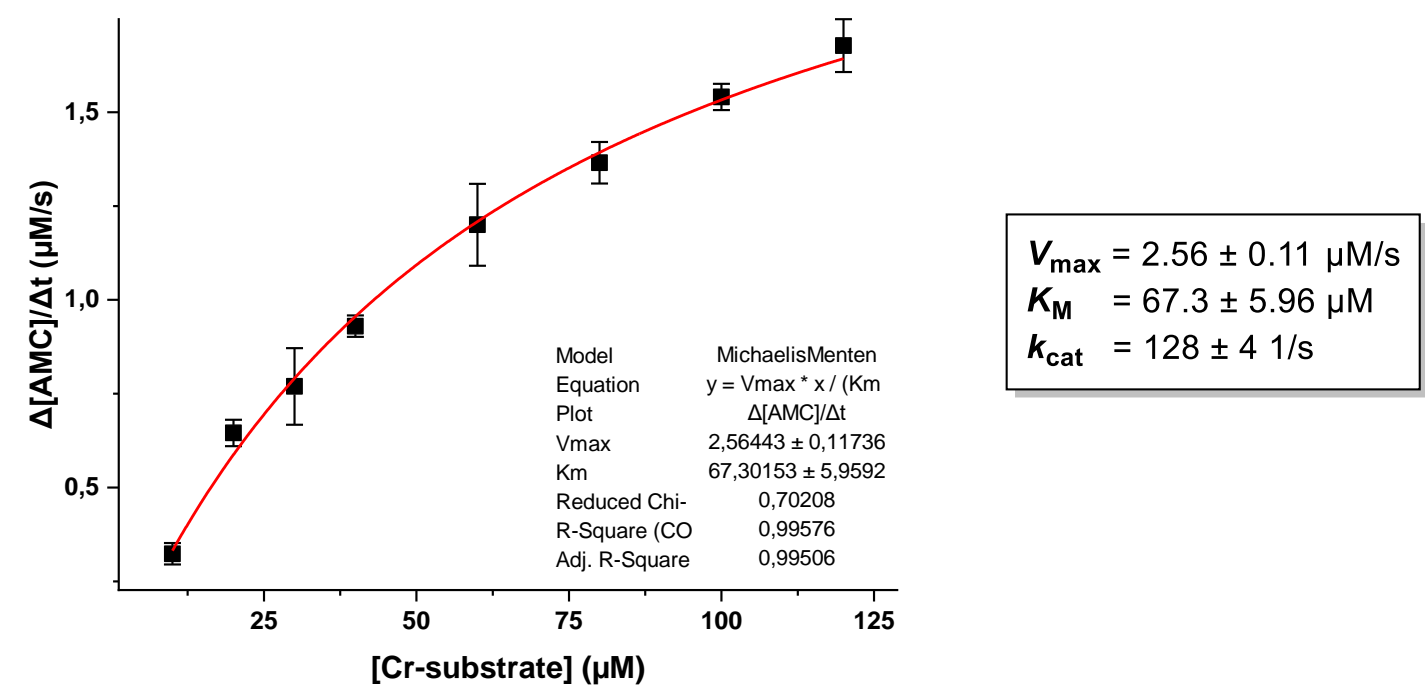

Figure S63. Exemplary results of the Michaelis-Menten analysis of the Cr/AAPF-AMC system.

\subsection{Determination of the Inhibition Constants of PIs}

\subsubsection{General Procedure}

Based on the constants obtained from Michaelis-Menten analysis of the chymotrypsin/Suc-AAPF-AMC system, the PI inhibition constants $\left(K_{\mathrm{i}}\right)$ at thermally adapted and $370 \mathrm{~nm}$ photostationary state were determined by the performance of a second fluorogenic assay similar to the one described in 4.3. Fluorogenic assays were performed at fixed enzyme concentration with varied substrate concentration in the presence of the respective PI according to the pipetting scheme outlined in Table S3.

Table S3. Pipetting scheme for the $K_{\mathrm{i}}$ determination of PIs at thermally adapted and $370 \mathrm{~nm}$ photostationary state. All experiments were done as triplicates using the autoinjector of the Tecan SparkM10 plate reader for the addition of the Crenzyme to each well prior to the respective measurement. Buffer-DMSO was used instead of Buffer in case of low water solubility of the PI.

\begin{tabular}{|c|c|c|c|c|c|c|c|}
\hline $\begin{array}{c}{[\text { Cr-substrate }]_{\text {well }} /} \\
\mu \mathrm{M}\end{array}$ & $\begin{array}{c}\mathrm{V} \text { (Cr-substrate, } 400 \\
\mu \mathrm{M} \text { stock) } / \mu \mathrm{L}\end{array}$ & $\begin{array}{c}{[\text { Cr-enzyme }]_{\text {well }} /} \\
\mu \mathrm{M}\end{array}$ & $\begin{array}{c}\mathrm{V}(\mathrm{Cr} \text {-enzyme, } 0.4 \\
\mu \mathrm{M} \text { stock }) / \mu \mathrm{L}\end{array}$ & {$[\mathrm{PI}]_{\mathrm{well}} / \boldsymbol{\mu M}$} & $\begin{array}{c}\mathrm{V} \text { (PI, } 1 \text { mM stock) / } \\
\mu \mathrm{L}\end{array}$ & $\begin{array}{c}\text { Buffer or Buffer- } \\
\text { DMSO } / \mu \mathrm{L}\end{array}$ & Total Volume / $\mu \mathrm{L}$ \\
\hline 10 & $\overline{5}$ & 0.02 & 10 & 50 & 10 & 175 & 200 \\
\hline 20 & 10 & 0.02 & 10 & 50 & 10 & 170 & 200 \\
\hline 30 & 15 & 0.02 & 10 & 50 & 10 & 165 & 200 \\
\hline 40 & 20 & 0.02 & 10 & 50 & 10 & 160 & 200 \\
\hline 60 & 30 & 0.02 & 10 & 50 & 10 & 150 & 200 \\
\hline 80 & 40 & 0.02 & 10 & 50 & 10 & 140 & 200 \\
\hline 100 & 50 & 0.02 & 10 & 50 & 10 & 130 & 200 \\
\hline 120 & 60 & 0.02 & 10 & 50 & 10 & 120 & 200 \\
\hline
\end{tabular}

All compounds except chymotrypsin have been pipetted using multichannel pipettes. The enzyme chymotrypsin was then added to the respective well of the 96 well-plate using the Tecan SparkM10 autoinjector. All experiments were done as triplicates. The fluorescence intensity of wells within 96well plates (purchased from Greiner Bio-One), containing $200 \mu \mathrm{L}$ of the respective solution, was monitored for $40 \mathrm{~s}$ (shaking 3s/orbital mode/amplitude $4 \mathrm{~mm}$ ) at $25^{\circ} \mathrm{C}$ using top fluorescence reading mode $\left(\lambda_{\mathrm{ex}} / \lambda_{\mathrm{em}}=380 \mathrm{~nm} / 460 \mathrm{~nm}\right.$ ), as described in the General Information (see 1.1) part of this supplementary information. Once the $K_{\mathrm{i}}$ for one isomer has been determined, $2 \mathrm{~mL}$ of the same PI stock 
solution were irradiated with UV-A light from a Avonec LED ( $\left.3 \mathrm{~W}, \lambda_{\max }=365 \mathrm{~nm}\right)$ for $10 \mathrm{~min}$ to reach the $365 \mathrm{~nm}$ photostationary state. In both cases, the amount of fluorescent product was calculated according to the AMC calibration curve (4.2). Based on the initial rate of enzymatic cleavage (v), the inhibition constant $K_{\mathrm{i}}$ was then obtained from fitting a Michaelis-Menten-type equation for competitive inhibition (similar to Eq. 1) to the experimental data using the Origin 2018b software package.

\subsubsection{Michaelis-Menten Plots and $K_{\mathrm{i}}$ of PIs}

Cr-PI and results

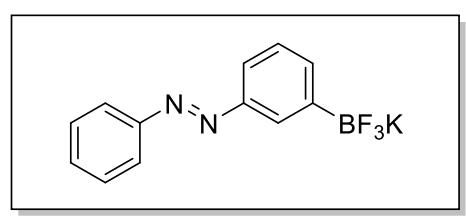

$V_{\max }=3.0441 \pm 0.1845 \mu \mathrm{M} / \mathrm{s}$

$$
K_{\mathrm{M}}=66.6 \pm 6.7 \mu \mathrm{M}
$$

$K_{\mathrm{i}}$ (thermally adapted) $=3.1 \pm 0.04 \mu \mathrm{M}$

$K_{\mathrm{i}}(365 \mathrm{~nm}$ PSS $)=17.1 \pm 0.57 \mu \mathrm{M}$

Switching Factor $=5.5 \pm 0.2$

$50 \mu \mathrm{M}$ Cr-PI (thermally adapted)

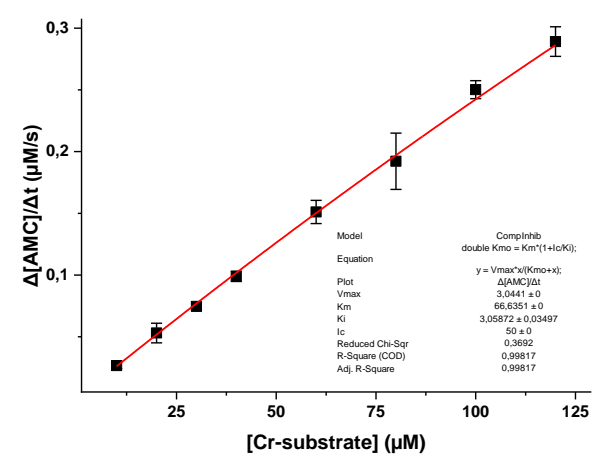

No Inhibitor

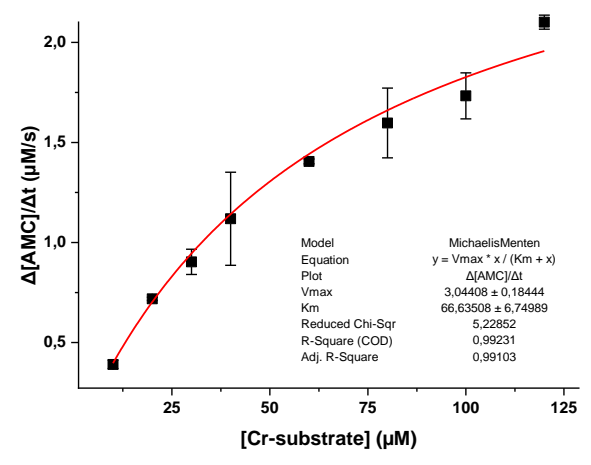

$50 \mu \mathrm{M}$ Cr-PI (365 nm PSS)

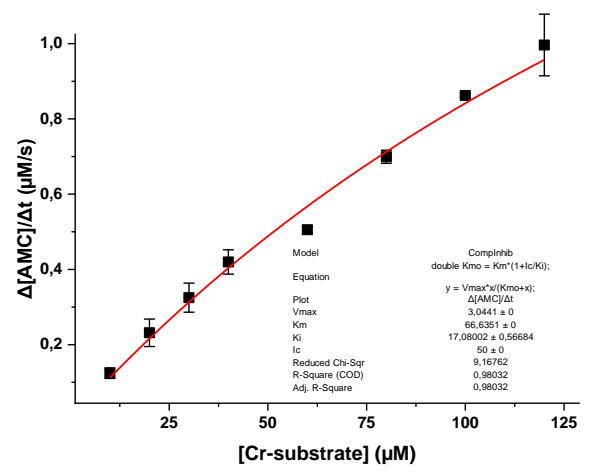

Figure S64. Michaelis-Menten plot and determined $K_{\mathrm{i}}$ values of PI 1 at thermally adapted state and $365 \mathrm{~nm}$ PSS. 

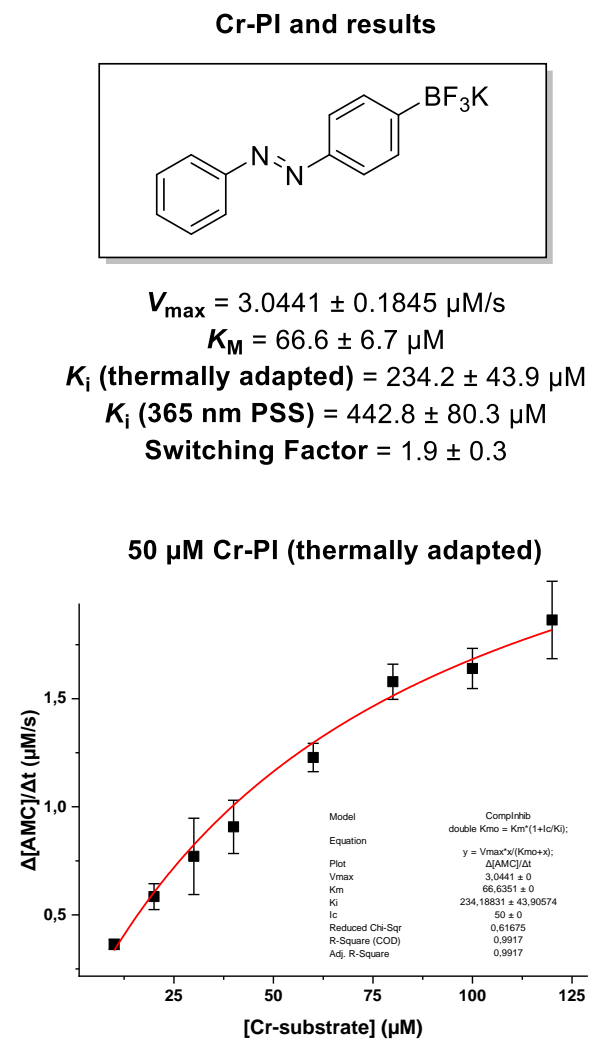

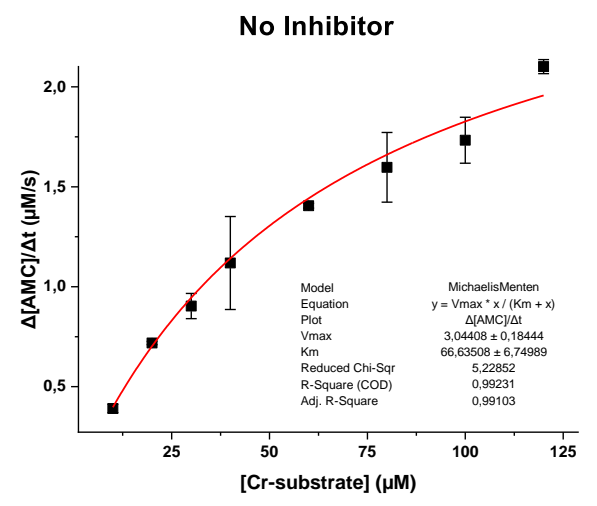

$50 \mu \mathrm{M}$ Cr-PI (365 nm PSS)

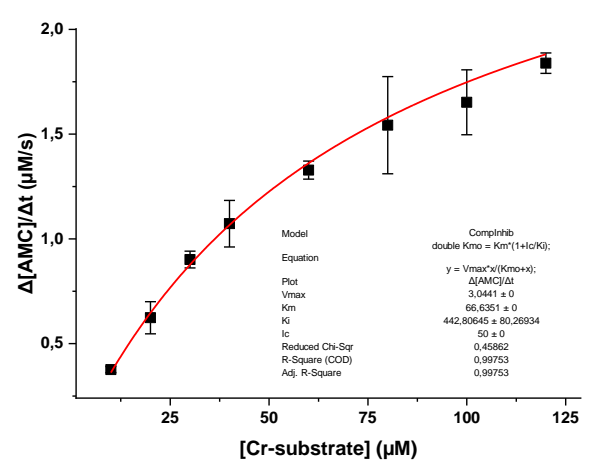

Figure S65. Michaelis-Menten plot and determined $K_{\mathrm{i}}$ values of PI 2 at thermally adapted state and $365 \mathrm{~nm}$ PSS.
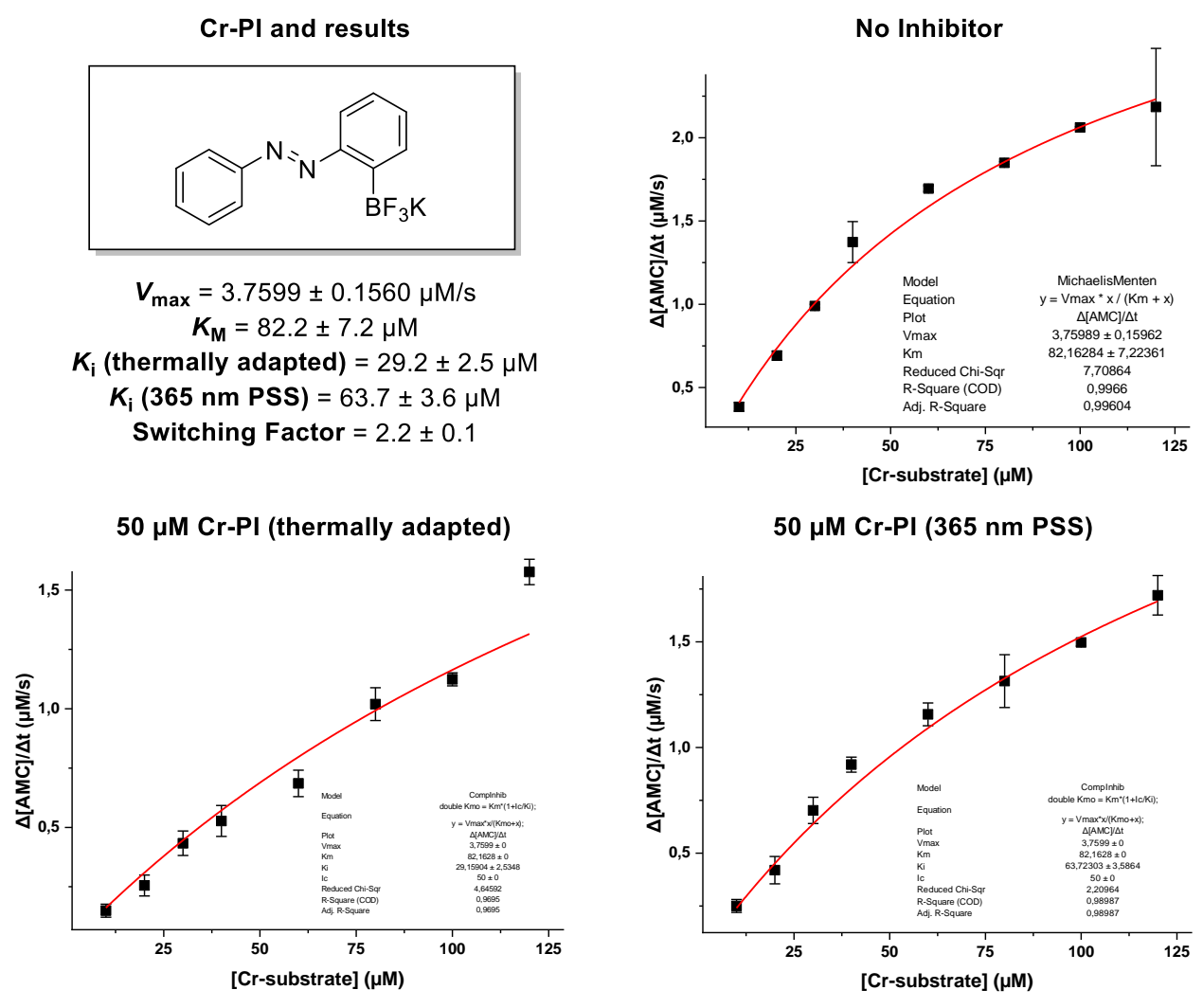

$50 \mu \mathrm{M}$ Cr-PI (365 nm PSS)

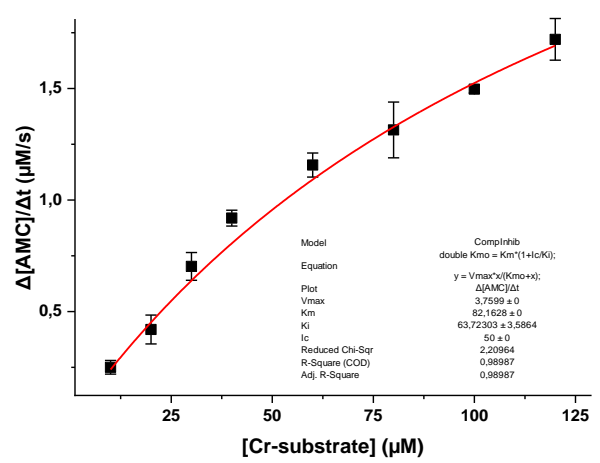

Figure S66. Michaelis-Menten plot and determined $K_{\mathrm{i}}$ values of PI 3 at thermally adapted state and 365 nm PSS. 
Cr-PI and results

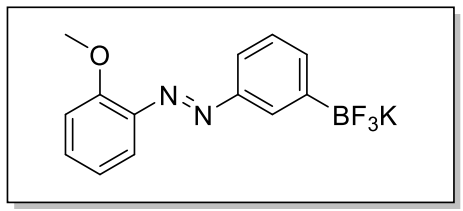

$V_{\max }=3.7599 \pm 0.1560 \mu \mathrm{M} / \mathrm{s}$

$$
K_{\mathrm{M}}=82.2 \pm 7.2 \mu \mathrm{M}
$$

$K_{\mathrm{i}}($ thermally adapted $)=49.9 \pm 4.1 \mu \mathrm{M}$

$\boldsymbol{K}_{\mathrm{i}}(\mathbf{3 6 5} \mathrm{nm}$ PSS $)=113.9 \pm 7.6 \mu \mathrm{M}$

Switching Factor $=2.3 \pm 0.1$

$50 \mu \mathrm{M}$ Cr-PI (thermally adapted)

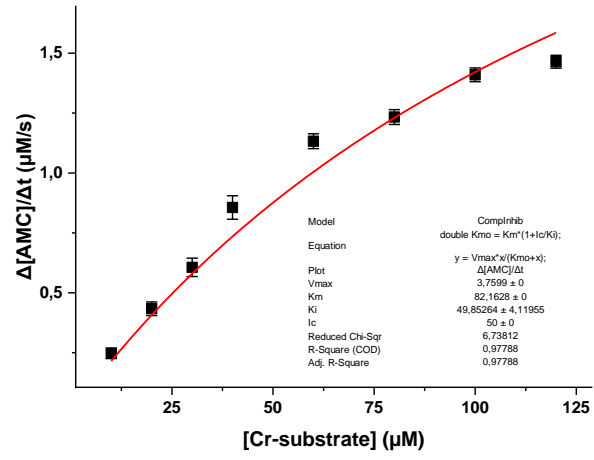

No Inhibitor

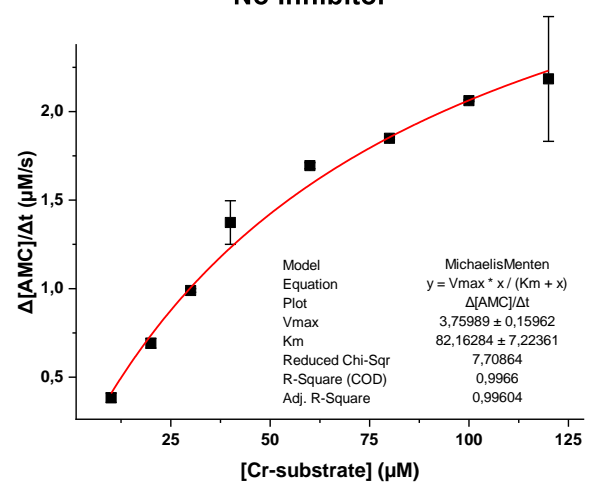

$50 \mu \mathrm{M}$ Cr-PI (365 nm PSS)

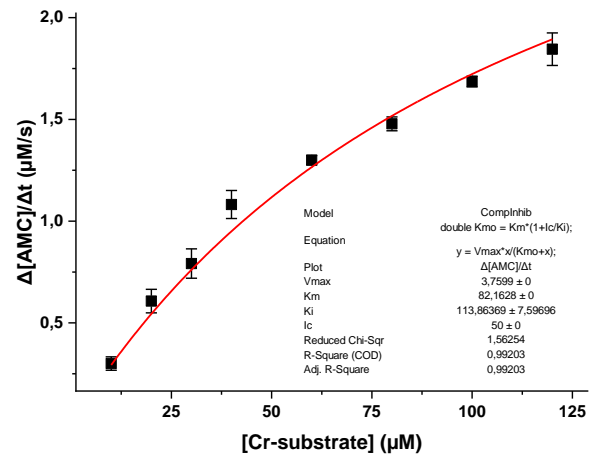

Figure S67. Michaelis-Menten plot and determined $K_{\mathrm{i}}$ values of PI 4 at thermally adapted state and $365 \mathrm{~nm}$ PSS.

Cr-PI and results

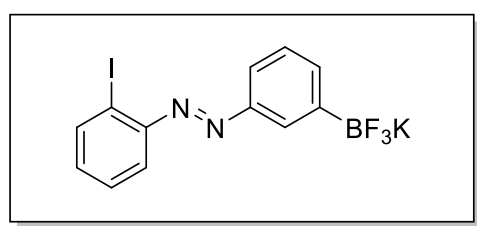

$V_{\max }=3.3949 \pm 0.1331 \mu \mathrm{M} / \mathrm{s}$

$K_{\mathrm{M}}=81.4 \pm 6.2 \mu \mathrm{M}$

$K_{\mathrm{i}}$ (thermally adapted) $=50.2 \pm 1.7 \mu \mathrm{M}$

$K_{\mathrm{i}}(365 \mathrm{~nm}$ PSS $)=132.9 \pm 18.1 \mu \mathrm{M}$

Switching Factor $=2.6 \pm 0.4$

$50 \mu \mathrm{M}$ Cr-PI (thermally adapted)

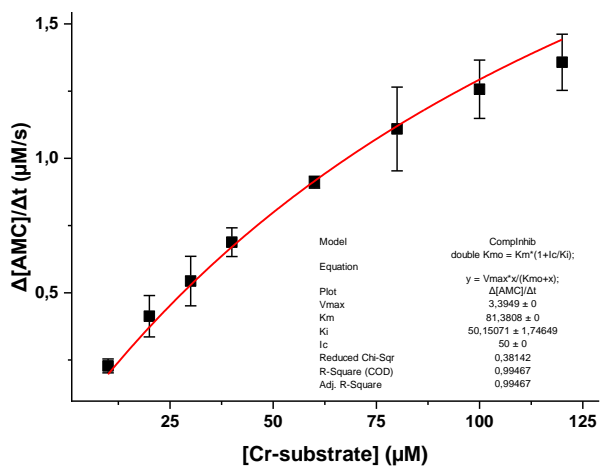

No Inhibitor

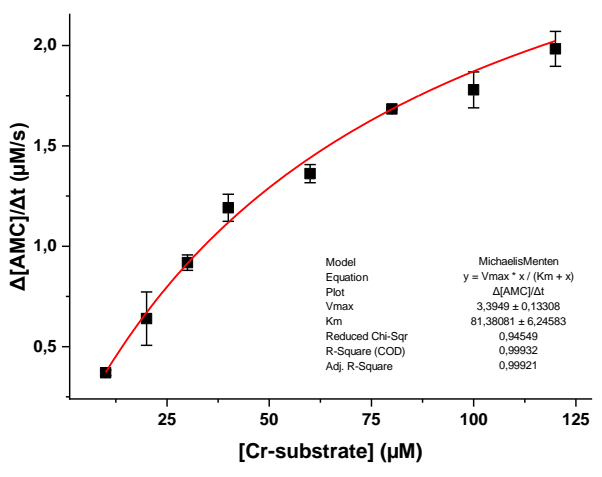

$50 \mu \mathrm{M}$ Cr-PI (365 nm PSS)

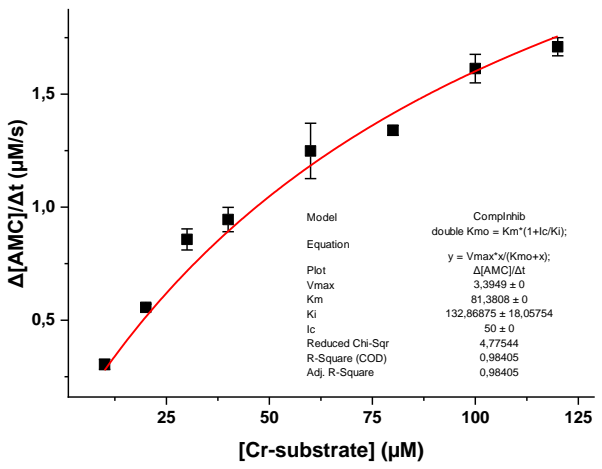

Figure S68. Michaelis-Menten plot and determined $K_{\mathrm{i}}$ values of PI 5 at thermally adapted state and $365 \mathrm{~nm}$ PSS. 
Cr-PI and results

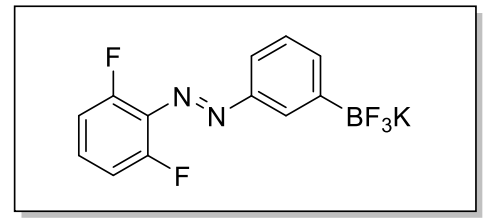

$V_{\text {max }}=3.3949 \pm 0.1331 \mu \mathrm{M} / \mathrm{s}$ $K_{\mathrm{M}}=81.4 \pm 6.2 \mu \mathrm{M}$

$K_{\mathrm{i}}$ (thermally adapted) $=32.0 \pm 0.6 \mu \mathrm{M}$ $K_{\mathrm{i}}(365 \mathrm{~nm}$ PSS $)=65.9 \pm 2.8 \mu \mathrm{M}$ Switching Factor $=2.1 \pm 0.1$

$50 \mu \mathrm{M}$ Cr-PI (thermally adapted)

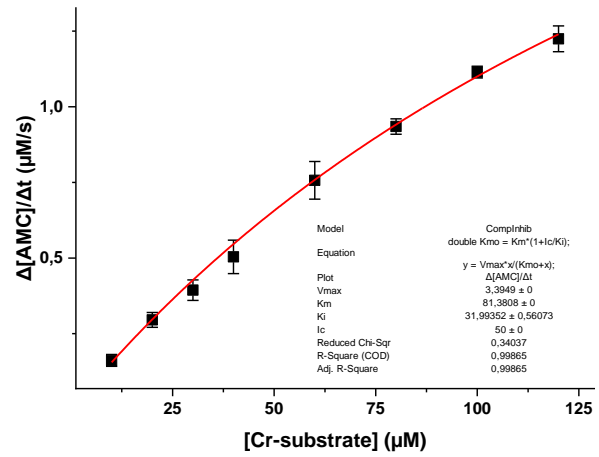

No Inhibitor

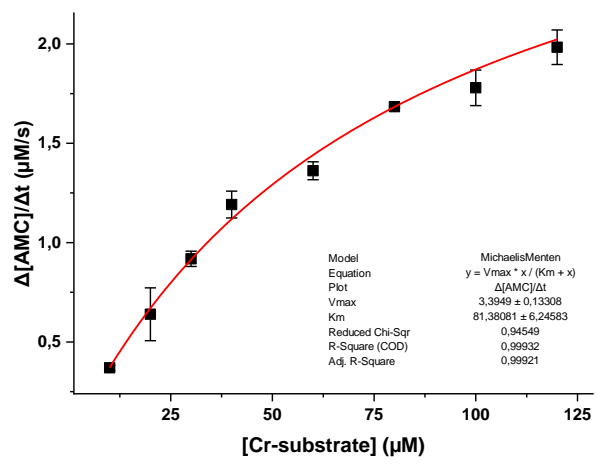

$50 \mu \mathrm{M}$ Cr-PI (365 nm PSS)

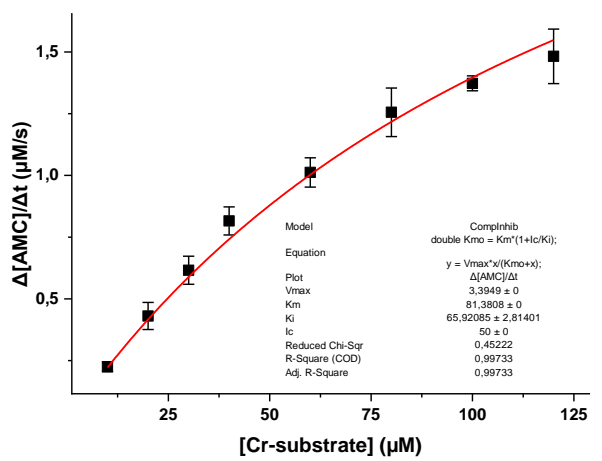

Figure S69. Michaelis-Menten plot and determined $K_{\mathrm{i}}$ values of PI 6 at thermally adapted state and $365 \mathrm{~nm}$ PSS.

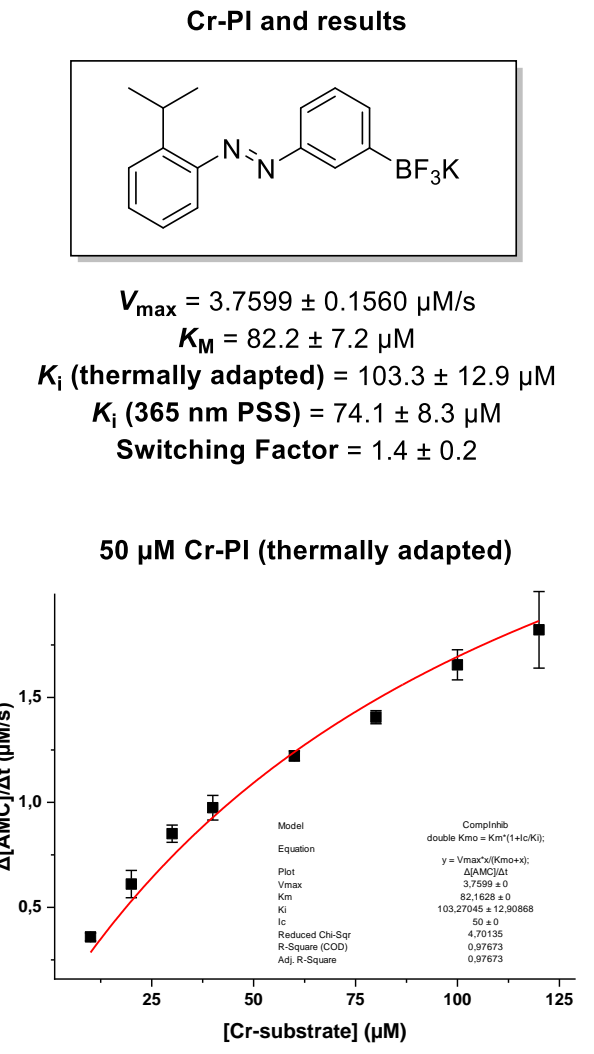

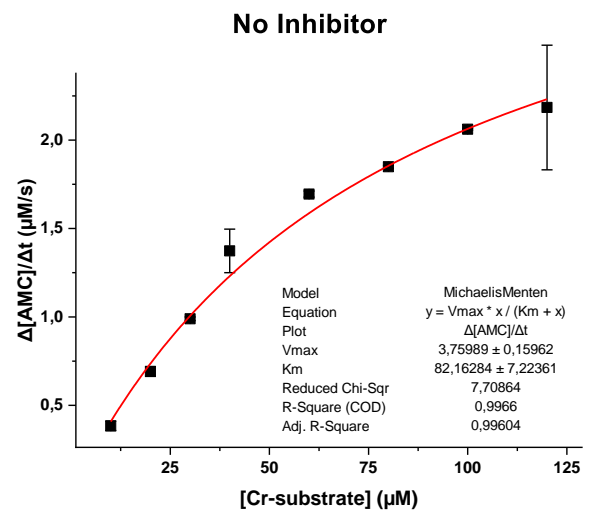

$50 \mu \mathrm{M}$ Cr-PI (365 nm PSS)

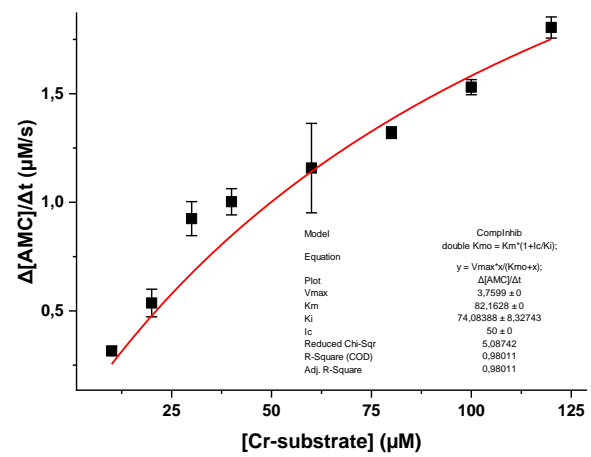

Figure S70. Michaelis-Menten plot and determined $K_{\mathrm{i}}$ values of PI 7 at thermally adapted state and $365 \mathrm{~nm}$ PSS. 
Cr-PI and results

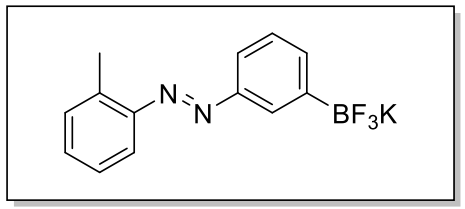

$V_{\text {max }}=3.7599 \pm 0.1560 \mu \mathrm{M} / \mathrm{s}$

$K_{\mathrm{M}}=82.2 \pm 7.2 \mu \mathrm{M}$

$K_{\mathrm{i}}($ thermally adapted $)=10.0 \pm 0.4 \mu \mathrm{M}$

$K_{\mathrm{i}}(365 \mathrm{~nm}$ PSS $)=63.6 \pm 2.5 \mu \mathrm{M}$

Switching Factor $=6.4 \pm 0.2$

$50 \mu \mathrm{M}$ Cr-PI (thermally adapted)

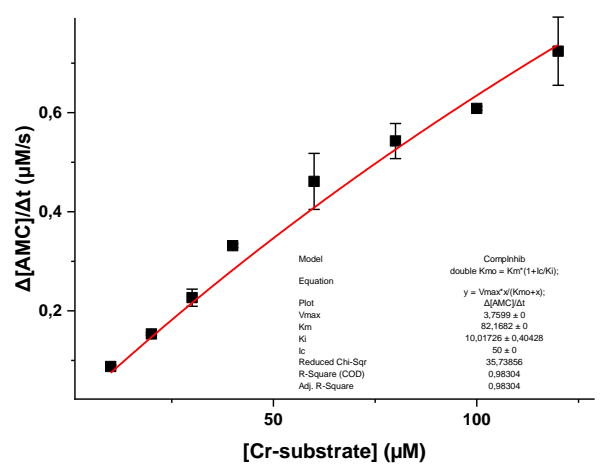

No Inhibitor

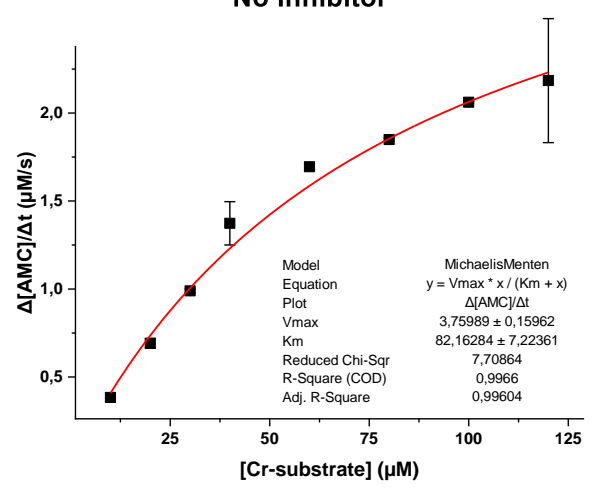

$50 \mu \mathrm{M}$ Cr-PI (365 nm PSS)

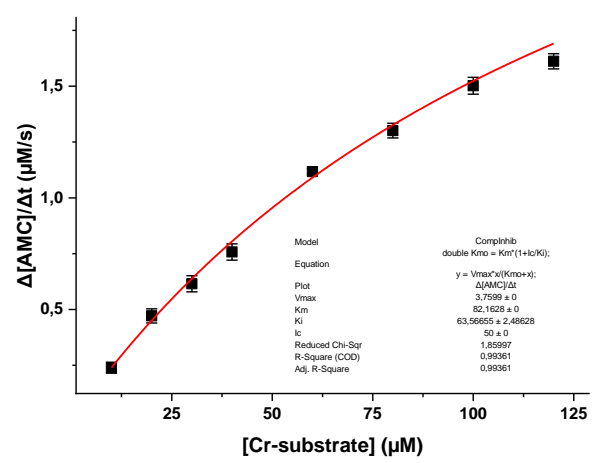

Figure S71. Michaelis-Menten plot and determined $K_{\mathrm{i}}$ values of PI $\mathbf{8}$ at thermally adapted state and $365 \mathrm{~nm}$ PSS.

Cr-PI and results

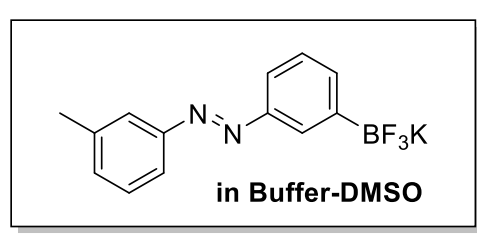

$V_{\text {max }}=2.5088 \pm 0.0936 \mu \mathrm{M} / \mathrm{s}$

$K_{\mathrm{M}}=62.7 \pm 4.8 \mu \mathrm{M}$

$K_{\mathrm{i}}($ thermally adapted $)=161.9 \pm 21.1 \mu \mathrm{M}$

$K_{\mathrm{i}}(365 \mathrm{~nm}$ PSS $)=212.1 \pm 44.1 \mu \mathrm{M}$

Switching Factor $=1.3 \pm 0.2$

$50 \mu \mathrm{M}$ Cr-PI (thermally adapted)

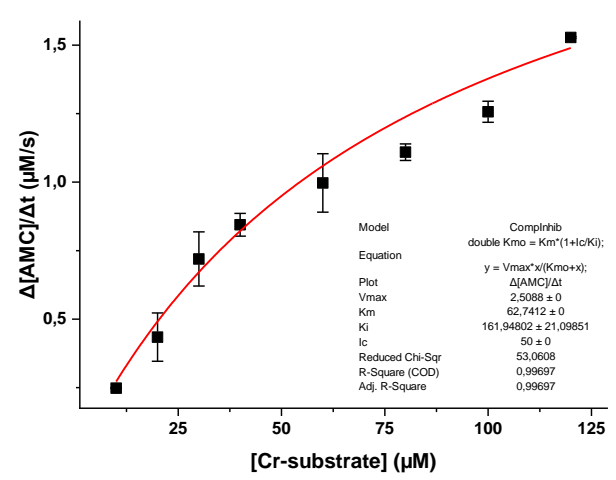

No Inhibitor

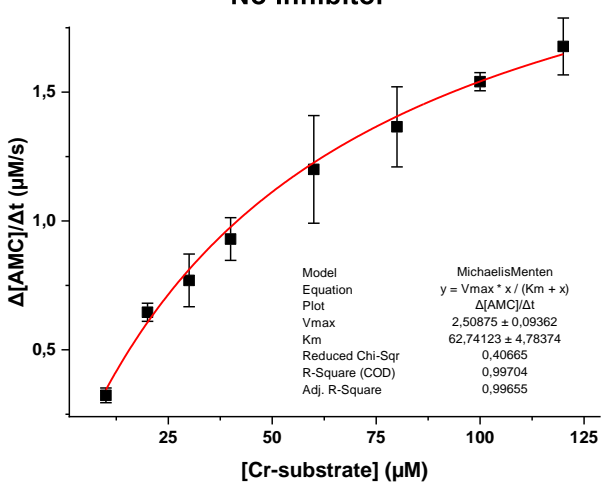

$50 \mu \mathrm{M}$ Cr-PI (365 nm PSS)

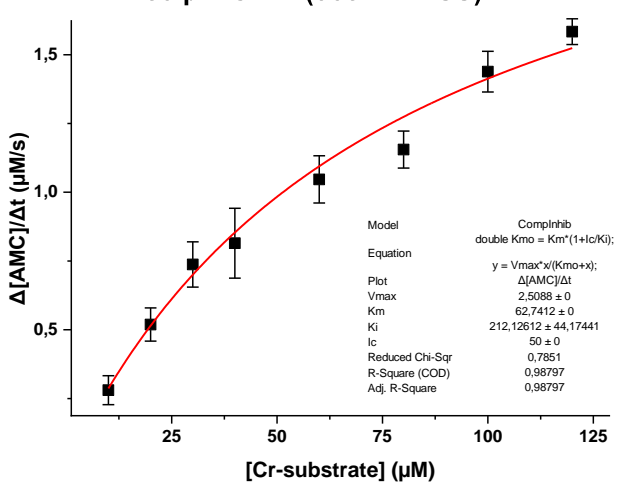

Figure S72. Michaelis-Menten plot and determined $K_{\mathrm{i}}$ values of PI 9 (in Buffer-DMSO) at thermally adapted state and $365 \mathrm{~nm}$ PSS. 

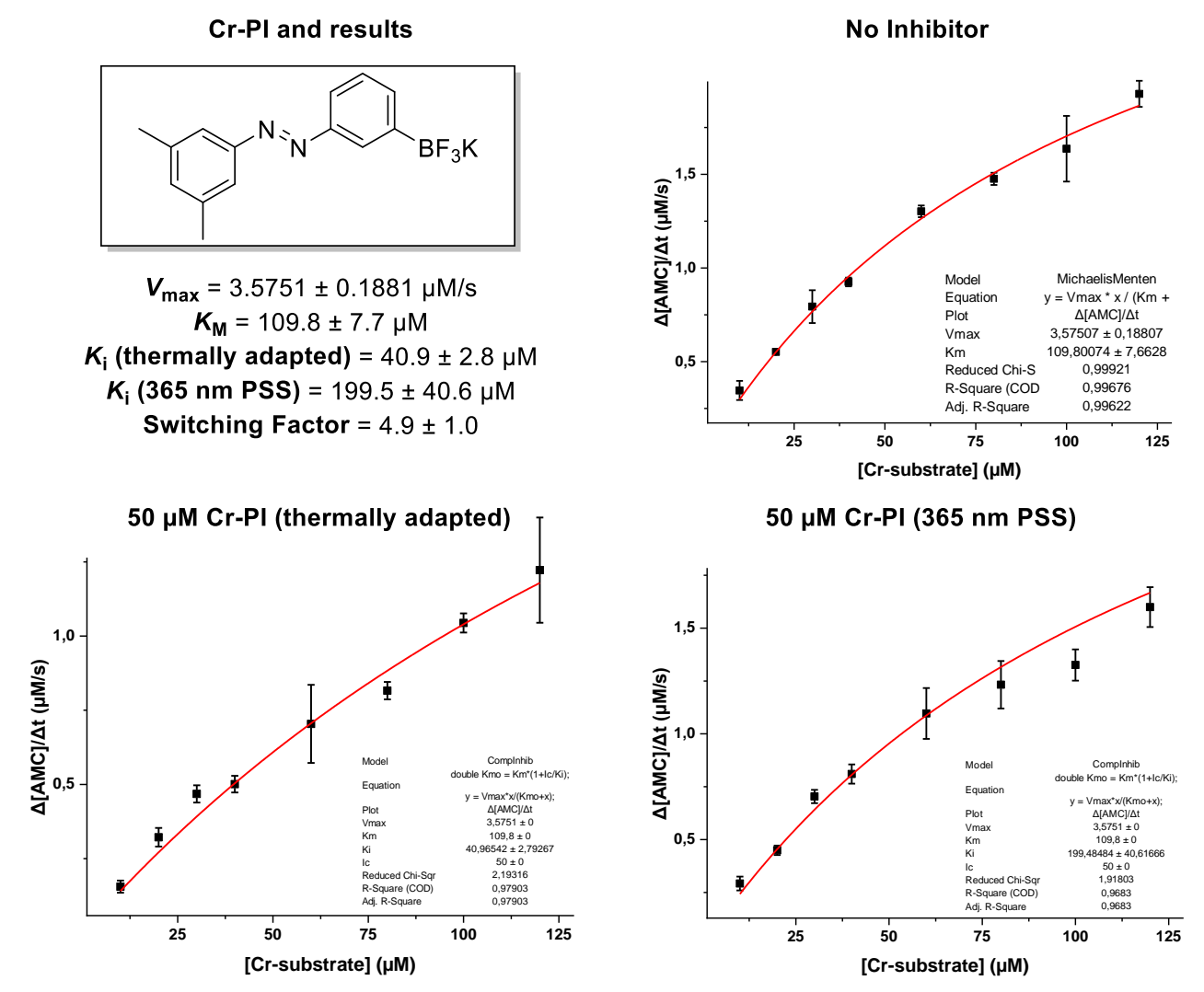

Figure S73. Michaelis-Menten plot and determined $K_{\mathrm{i}}$ values of PI 10 at thermally adapted state and $365 \mathrm{~nm}$ PSS.
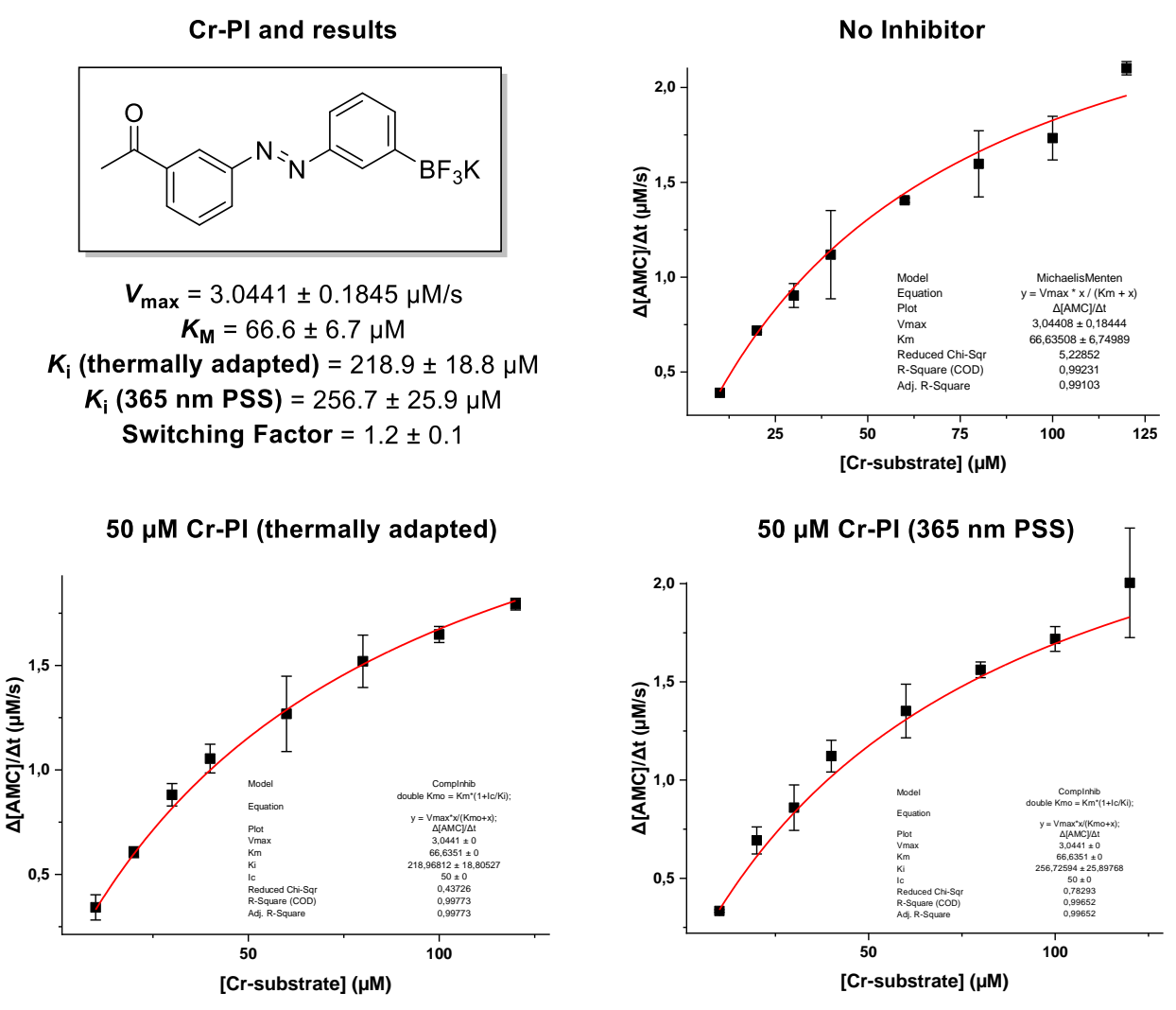

Figure S74. Michaelis-Menten plot and determined $K_{\mathrm{i}}$ values of PI 11 at thermally adapted state and $365 \mathrm{~nm}$ PSS. 

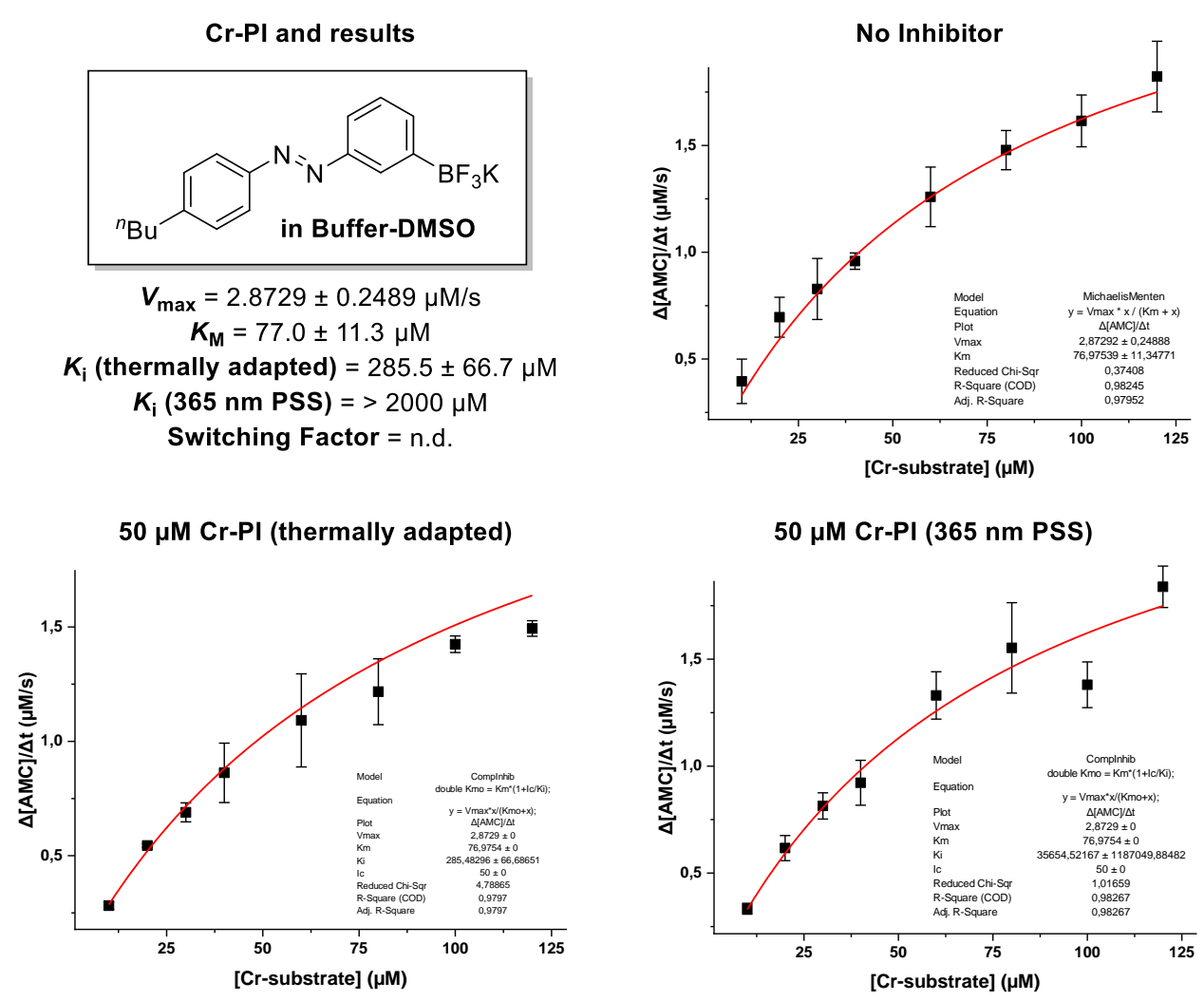

Figure S75. Michaelis-Menten plot and determined $K_{\mathrm{i}}$ values of PI 12 (in Buffer-DMSO) at thermally adapted state and $365 \mathrm{~nm}$ PSS.

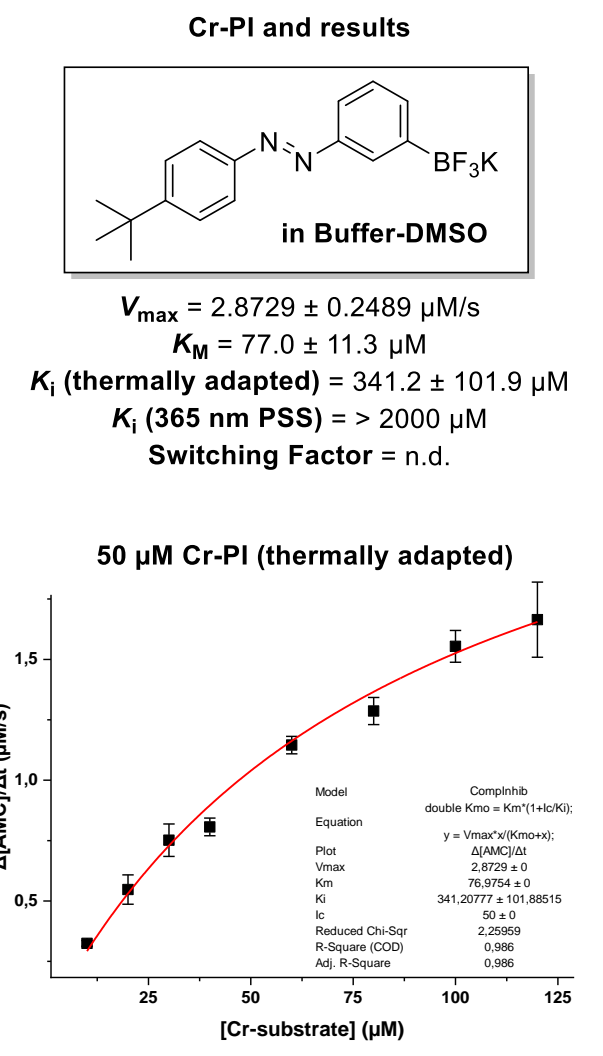

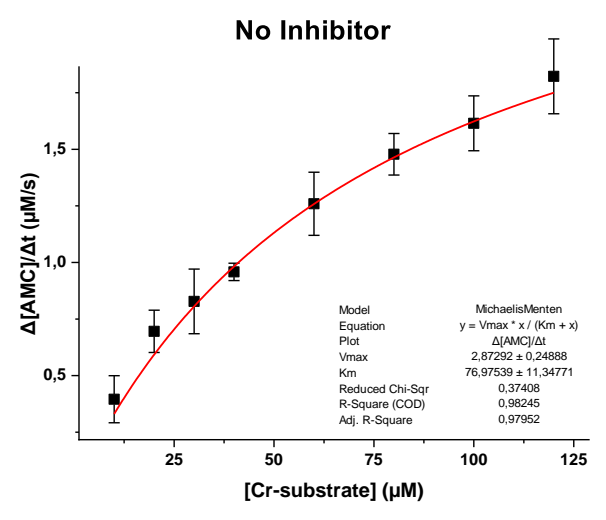

$50 \mu \mathrm{M}$ Cr-PI (365 nm PSS)

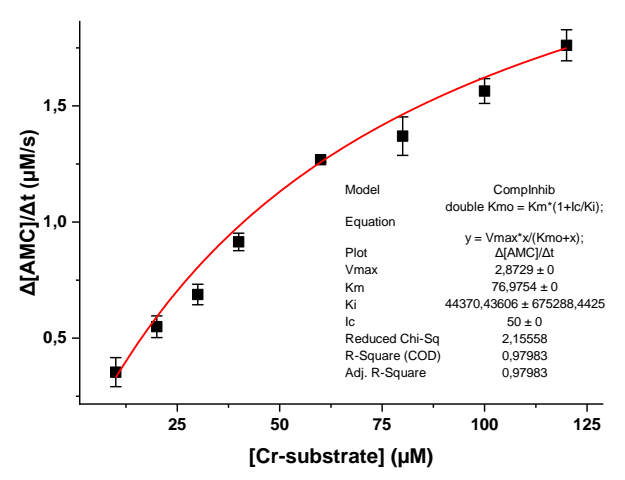

Figure S76. Michaelis-Menten plot and determined $K_{\mathrm{i}}$ values of PI 13 (in Buffer-DMSO) at thermally adapted state and $365 \mathrm{~nm}$ PSS. 
Cr-PI and results<smiles>O=C(O)c1ccc(/N=N/c2cccc(Br)c2)cc1</smiles>

$V_{\text {max }}=3.3949 \pm 0.1331 \mu \mathrm{M} / \mathrm{s}$ $K_{M}=81.4 \pm 6.2 \mu \mathrm{M}$

$K_{\mathrm{i}}($ thermally adapted $)=78.7 \pm 7.3 \mu \mathrm{M}$ $K_{\mathrm{i}}(365 \mathrm{~nm}$ PSS $)=554.2 \pm 195.2 \mu \mathrm{M}$

Switching Factor $=7 \pm 2.5$

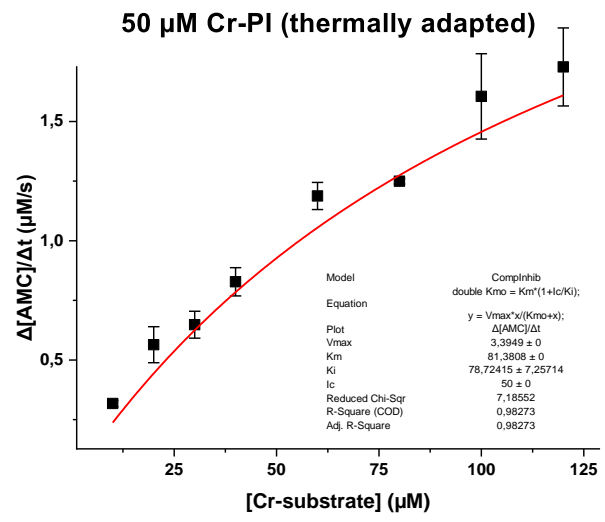

No Inhibitor

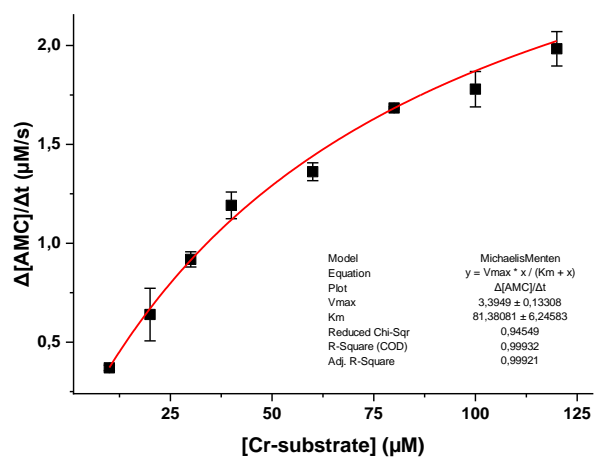

$50 \mu \mathrm{M}$ Cr-PI (365 nm PSS)

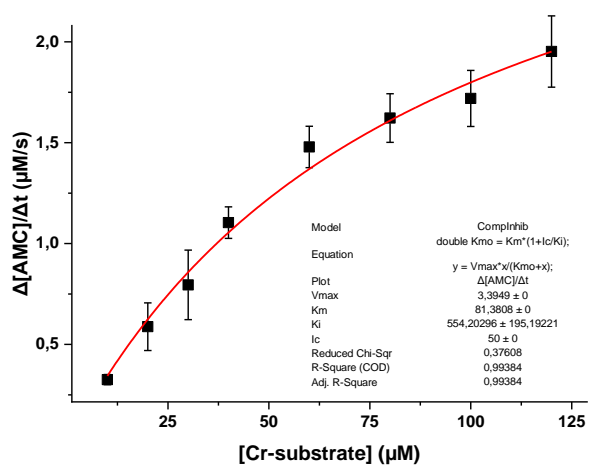

Figure S77. Michaelis-Menten plot and determined $K_{\mathrm{i}}$ values of PI 14 at thermally adapted state and $365 \mathrm{~nm}$ PSS.

Cr-PI and results<smiles>COC(=O)c1ccc(N=Nc2cccc(Br)c2)cc1</smiles>

$V_{\max }=2.8729 \pm 0.2489 \mu \mathrm{M} / \mathrm{s}$

$K_{\mathrm{M}}=77.0 \pm 11.3 \mu \mathrm{M}$

$K_{\mathrm{i}}$ (thermally adapted) $=226.2 \pm 27.2 \mu \mathrm{M}$

$K_{\mathrm{i}}(365 \mathrm{~nm}$ PSS) $=1224.9 \pm 654.7 \mu \mathrm{M}$

Switching Factor $=5.4 \pm 2.9$

$50 \mu \mathrm{M}$ Cr-PI (thermally adapted)

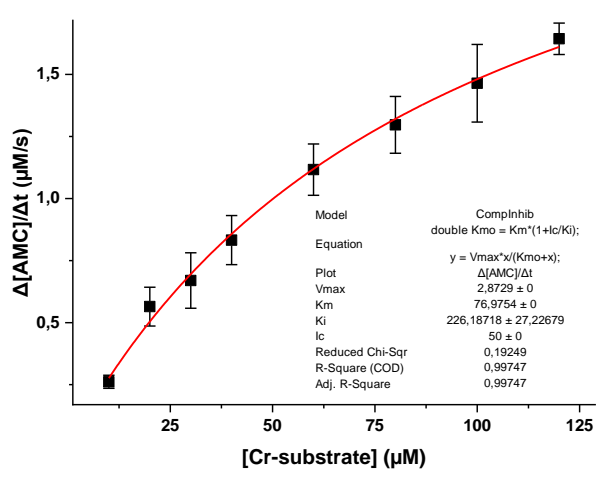

No Inhibitor

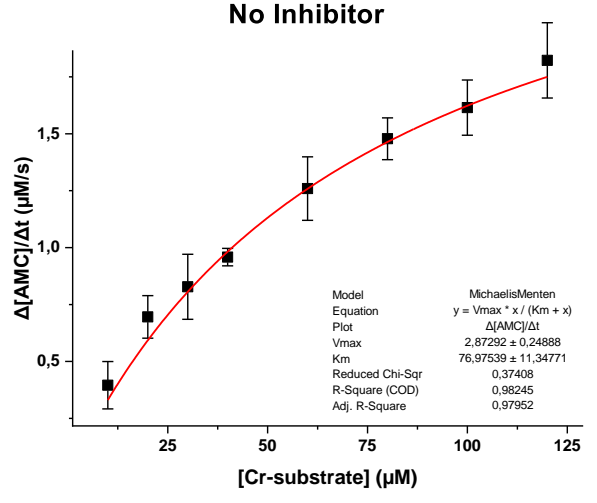

$50 \mu \mathrm{M}$ Cr-PI (365 nm PSS)

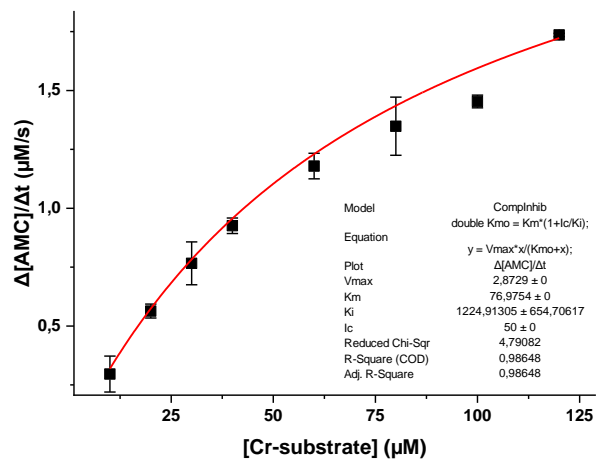

Figure S78. Michaelis-Menten plot and determined $K_{\mathrm{i}}$ values of PI 15 (in Buffer-DMSO) at thermally adapted state and $365 \mathrm{~nm}$ PSS. 
Cr-PI and results

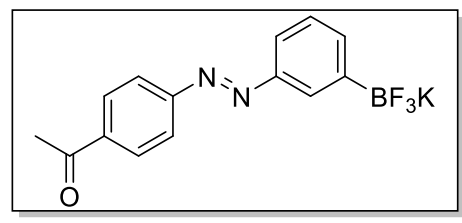

$V_{\max }=3.5751 \pm 0.1881 \mu \mathrm{M} / \mathrm{s}$

$K_{\mathrm{M}}=109.8 \pm 7.7 \mu \mathrm{M}$

$K_{\mathrm{i}}$ (thermally adapted $)=122.1 \pm 18.6 \mu \mathrm{M}$

$K_{\mathrm{i}}(365 \mathrm{~nm}$ PSS $)=292.0 \pm 60.0 \mu \mathrm{M}$

Switching Factor $=2.4 \pm 0.5$

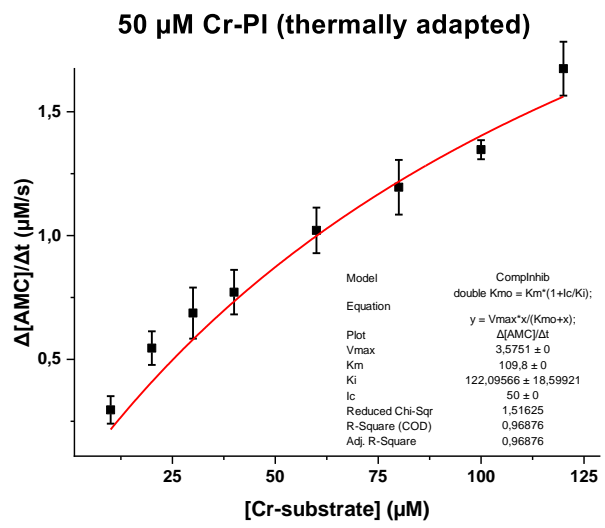

No Inhibitor

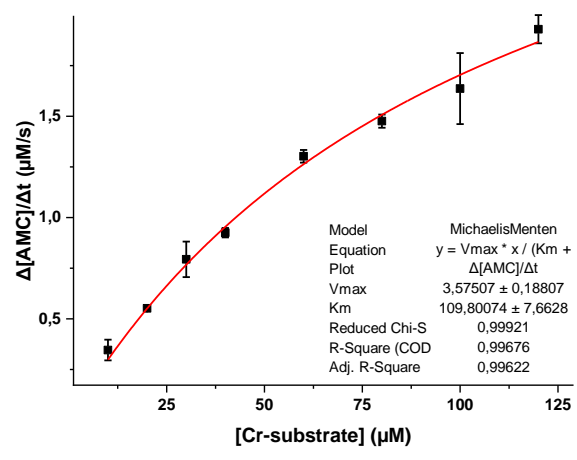

$50 \mu \mathrm{M}$ Cr-PI (365 nm PSS)

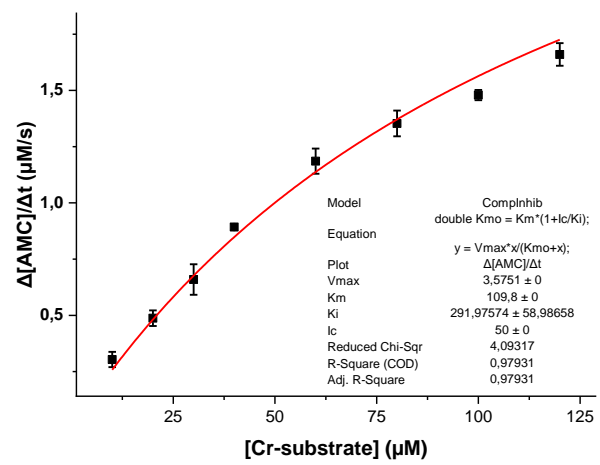

Figure S79. Michaelis-Menten plot and determined $K_{\mathrm{i}}$ values of PI 16 at thermally adapted state and $365 \mathrm{~nm}$ PSS.

$\mathrm{Cr}-\mathrm{PI}$ and results

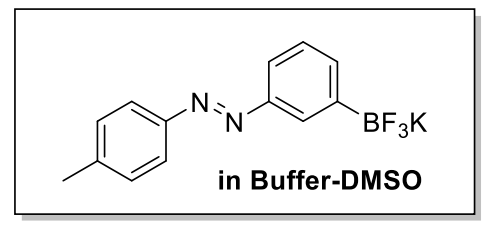

$V_{\max }=2.5088 \pm 0.0936 \mu \mathrm{M} / \mathrm{s}$

$K_{\mathrm{M}}=62.7 \pm 4.8 \mu \mathrm{M}$

$K_{\mathrm{i}}$ (thermally adapted) $=3.0 \pm 0.02 \mu \mathrm{M}$

$K_{\mathrm{i}}(365 \mathrm{~nm}$ PSS $)=35.8 \pm 1.51 \mu \mathrm{M}$

Switching Factor $=11.9 \pm 0.5$

$50 \mu \mathrm{M}$ Cr-PI (thermally adapted)

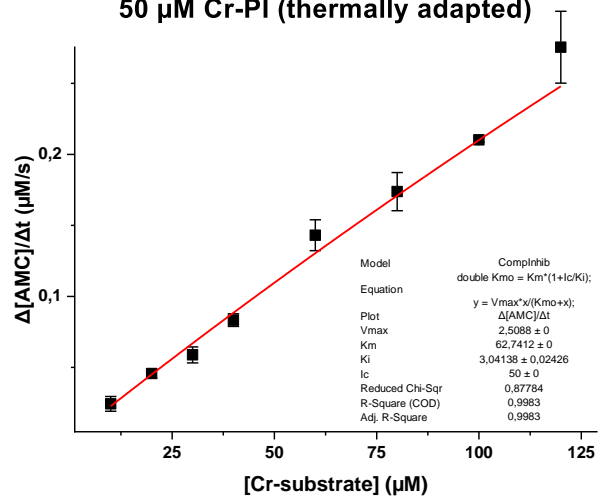

No Inhibitor

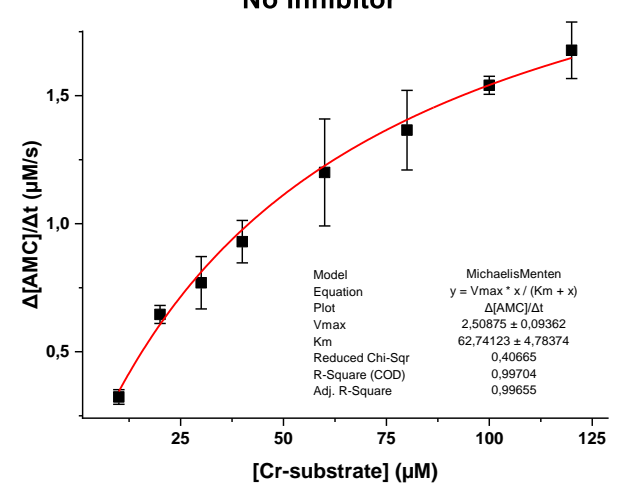

$50 \mu \mathrm{M}$ Cr-PI (365 nm PSS)

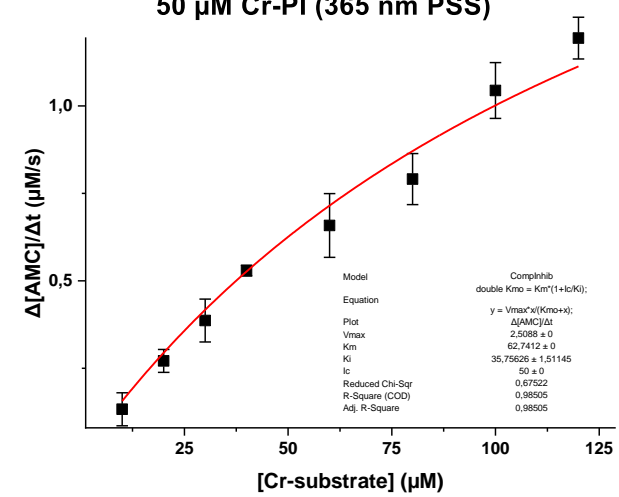

Figure S80. Michaelis-Menten plot and determined $K_{\mathrm{i}}$ values of PI 17 (in Buffer-DMSO) at thermally adapted state and $365 \mathrm{~nm}$ PSS. 

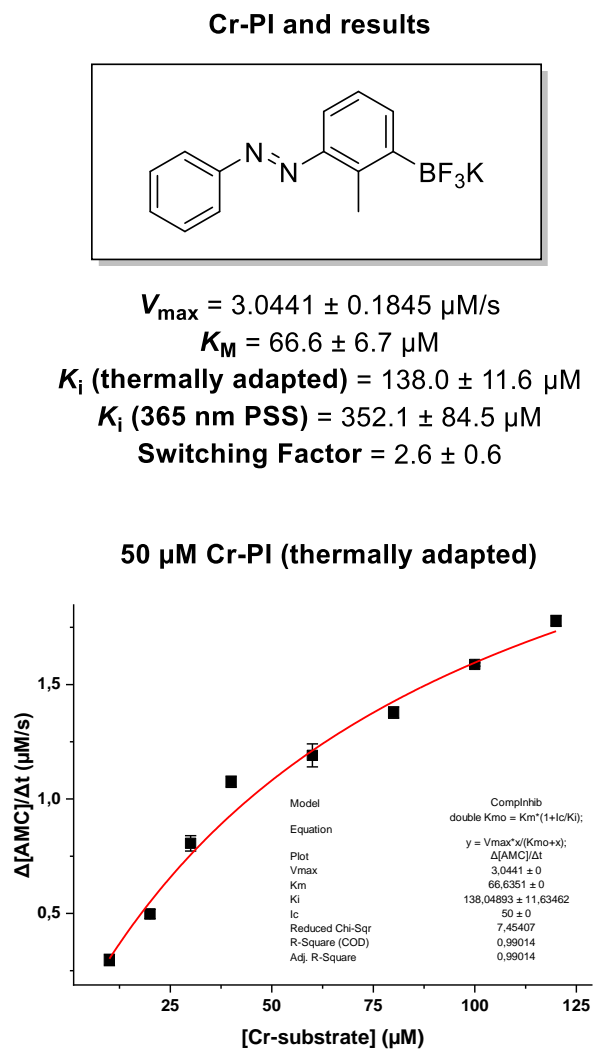

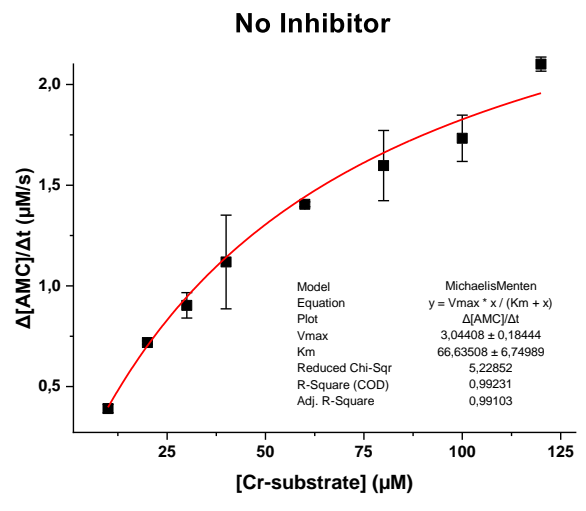

$50 \mu \mathrm{M}$ Cr-PI (365 nm PSS)

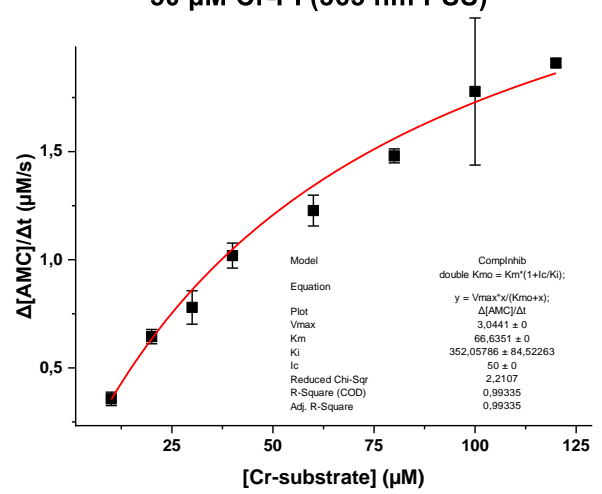

Figure S81. Michaelis-Menten plot and determined $K_{\mathrm{i}}$ values of PI 18 at thermally adapted state and $365 \mathrm{~nm}$ PSS.

Cr-PI and results

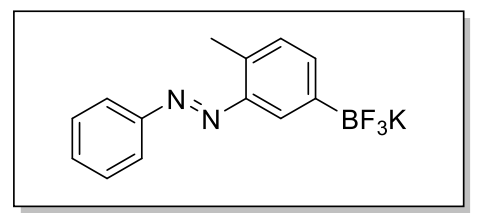

$V_{\max }=4.0767 \pm 0.251 \mu \mathrm{M} / \mathrm{s}$

$K_{\mathrm{M}}=121.1 \pm 9.5 \mu \mathrm{M}$

$K_{\mathrm{i}}$ (thermally adapted) $=5.8 \pm 0.2 \mu \mathrm{M}$

$K_{\mathrm{i}}(365 \mathrm{~nm}$ PSS $)=23.5 \pm 0.8 \mu \mathrm{M}$

Switching Factor $=4.0 \pm 0.2$

$50 \mu \mathrm{M} \mathrm{Cr}-\mathrm{PI}$ (thermally adapted)

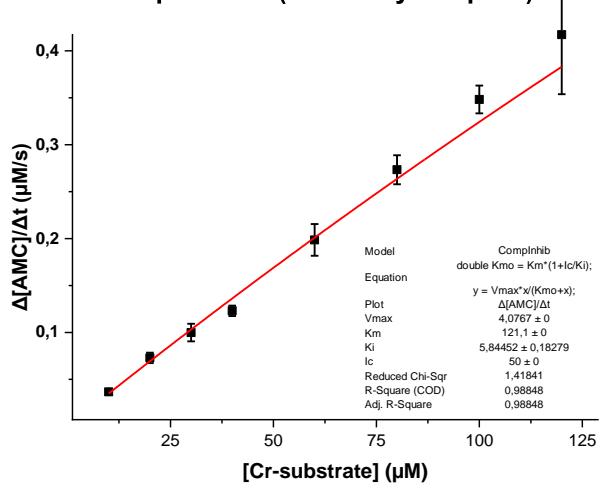

No Inhibitor

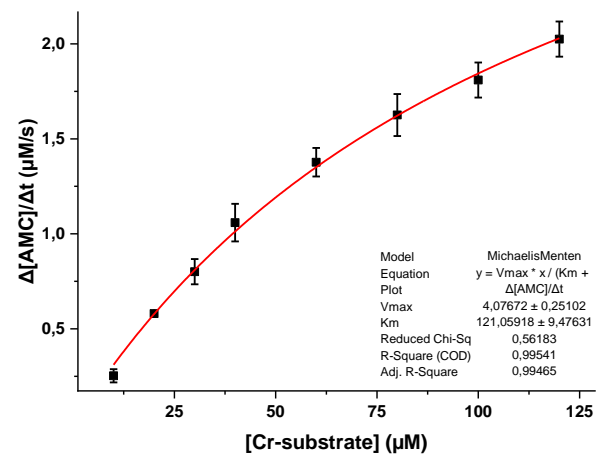

$50 \mu \mathrm{M}$ Cr-PI (365 nm PSS)

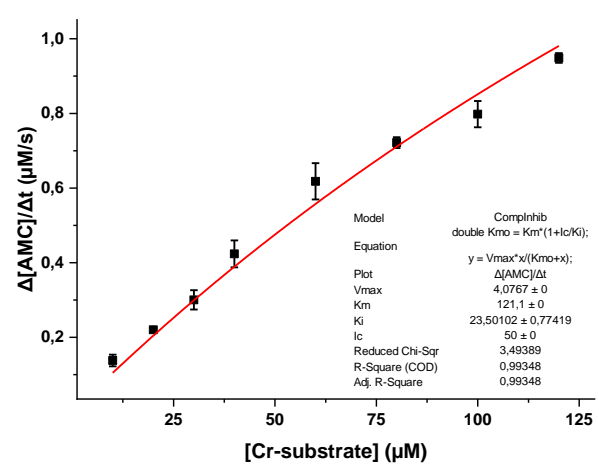

Figure S82. Michaelis-Menten plot and determined $K_{\mathrm{i}}$ values of PI 19 at thermally adapted state and $365 \mathrm{~nm}$ PSS. 
$\mathrm{Cr}-\mathrm{PI}$ and results

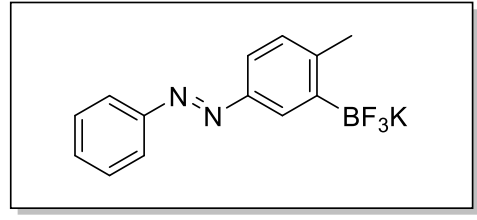

$V_{\max }=3.0339 \pm 0.1966 \mu \mathrm{M} / \mathrm{s}$

$K_{\mathrm{M}}=79.9 \pm 7.8 \mu \mathrm{M}$

$K_{\mathrm{i}}$ (thermally adapted) $=12.6 \pm 0.6 \mu \mathrm{M}$

$K_{\mathrm{i}}(365 \mathrm{~nm}$ PSS $)=264.2 \pm 50.6 \mu \mathrm{M}$

Switching Factor $=21.0 \pm 4$

$50 \mu \mathrm{M} \mathrm{Cr}$-PI (thermally adapted)

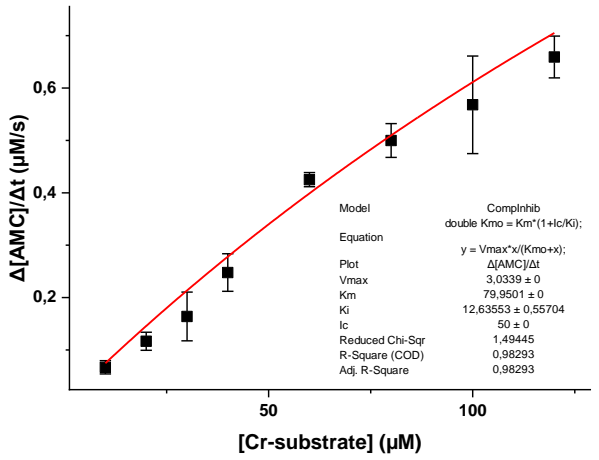

Cr-PI and results

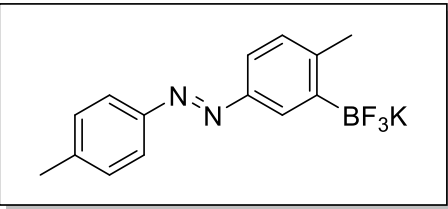

$V_{\max }=3.5751 \pm 0.1881 \mu \mathrm{M} / \mathrm{s}$

$K_{\mathrm{M}}=109.8 \pm 7.7 \mu \mathrm{M}$

$K_{\mathrm{i}}($ thermally adapted $)=200.4 \pm 17.2 \mu \mathrm{M}$

$K_{\mathrm{i}}(365 \mathrm{~nm}$ PSS $)=>2000 \mu \mathrm{M}$

Switching Factor $=$ n.d

$50 \mu \mathrm{M}$ Cr-PI (thermally adapted)

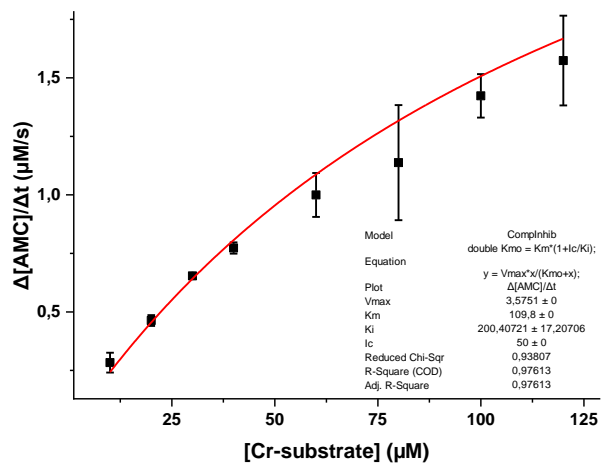

No Inhibitor

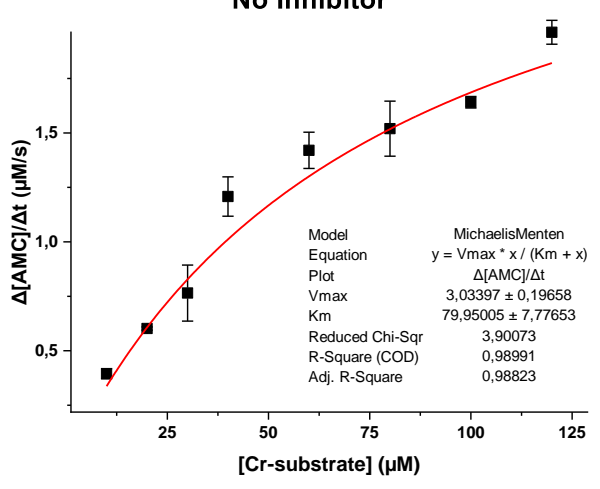

$50 \mu \mathrm{M}$ Cr-PI (365 nm PSS)

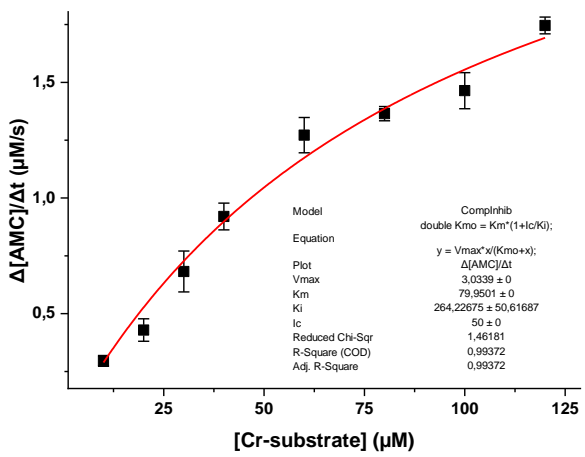

No Inhibitor

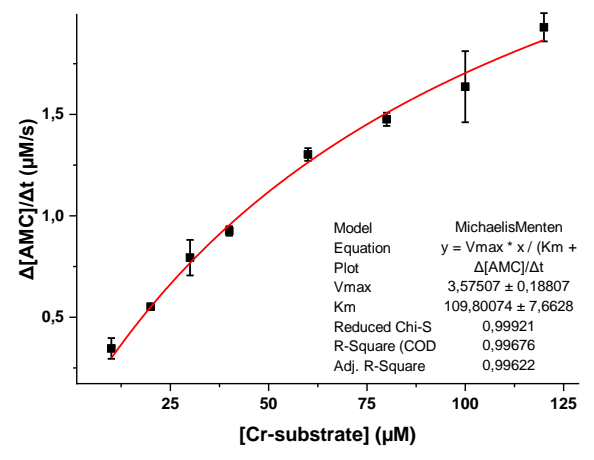

$50 \mu \mathrm{M}$ Cr-PI (365 nm PSS)

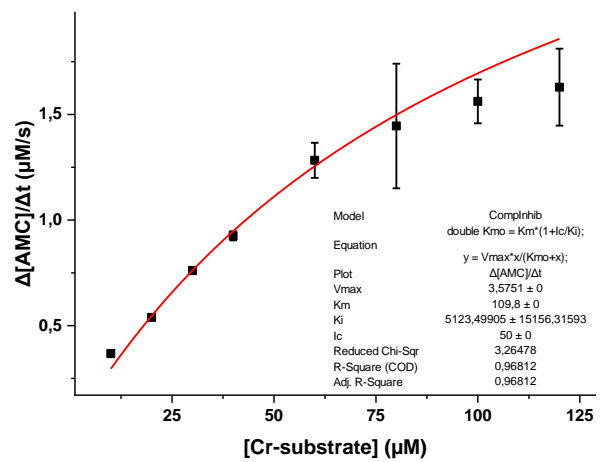

Figure S83. Michaelis-Menten plot and determined $K_{\mathrm{i}}$ values of PIs $\mathbf{2 0}$ and $\mathbf{2 1}$ at thermally adapted state and $365 \mathrm{~nm}$ PSS. 


\subsubsection{Overview of the Determined PI Inhibition Constants}

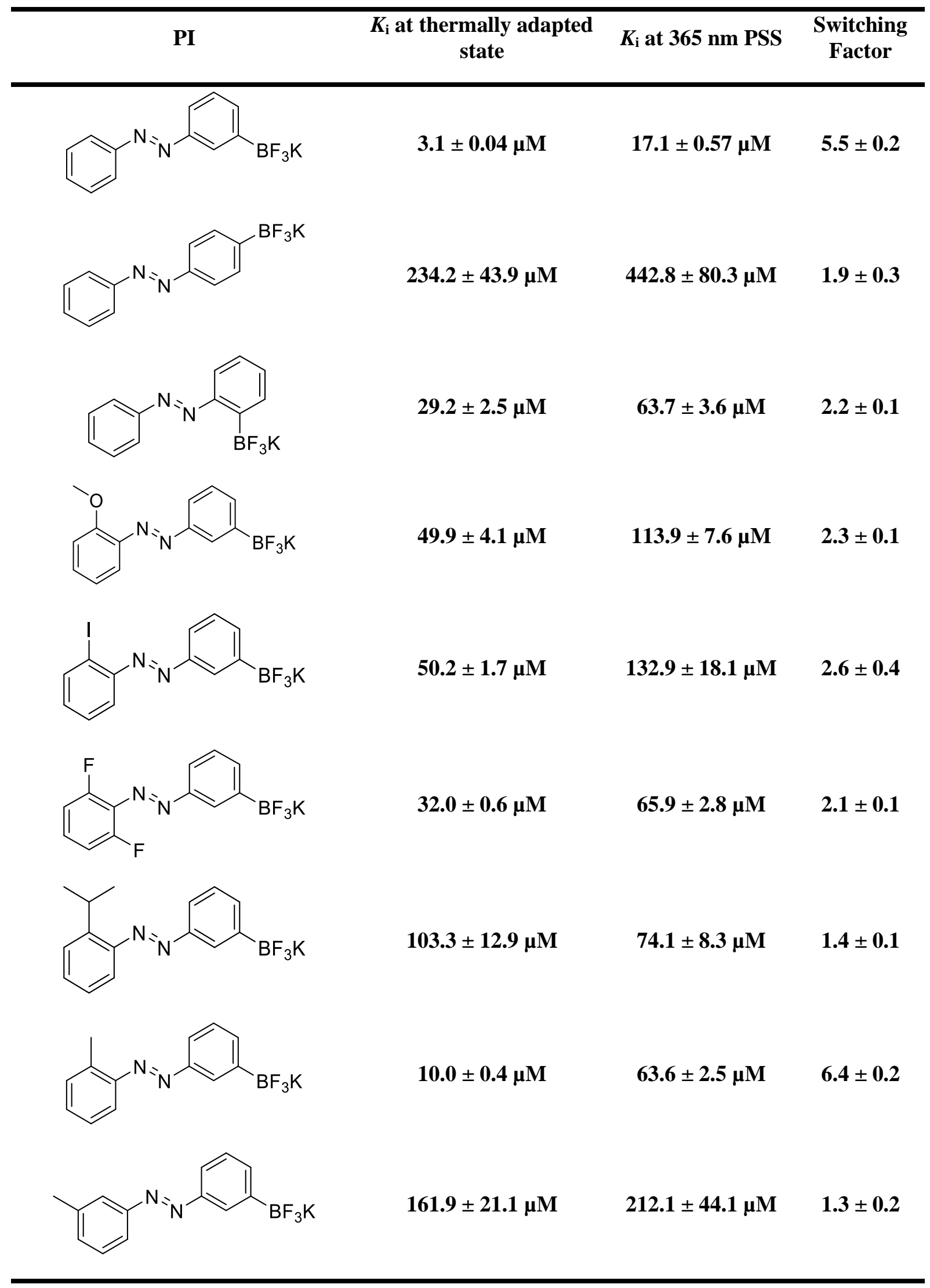




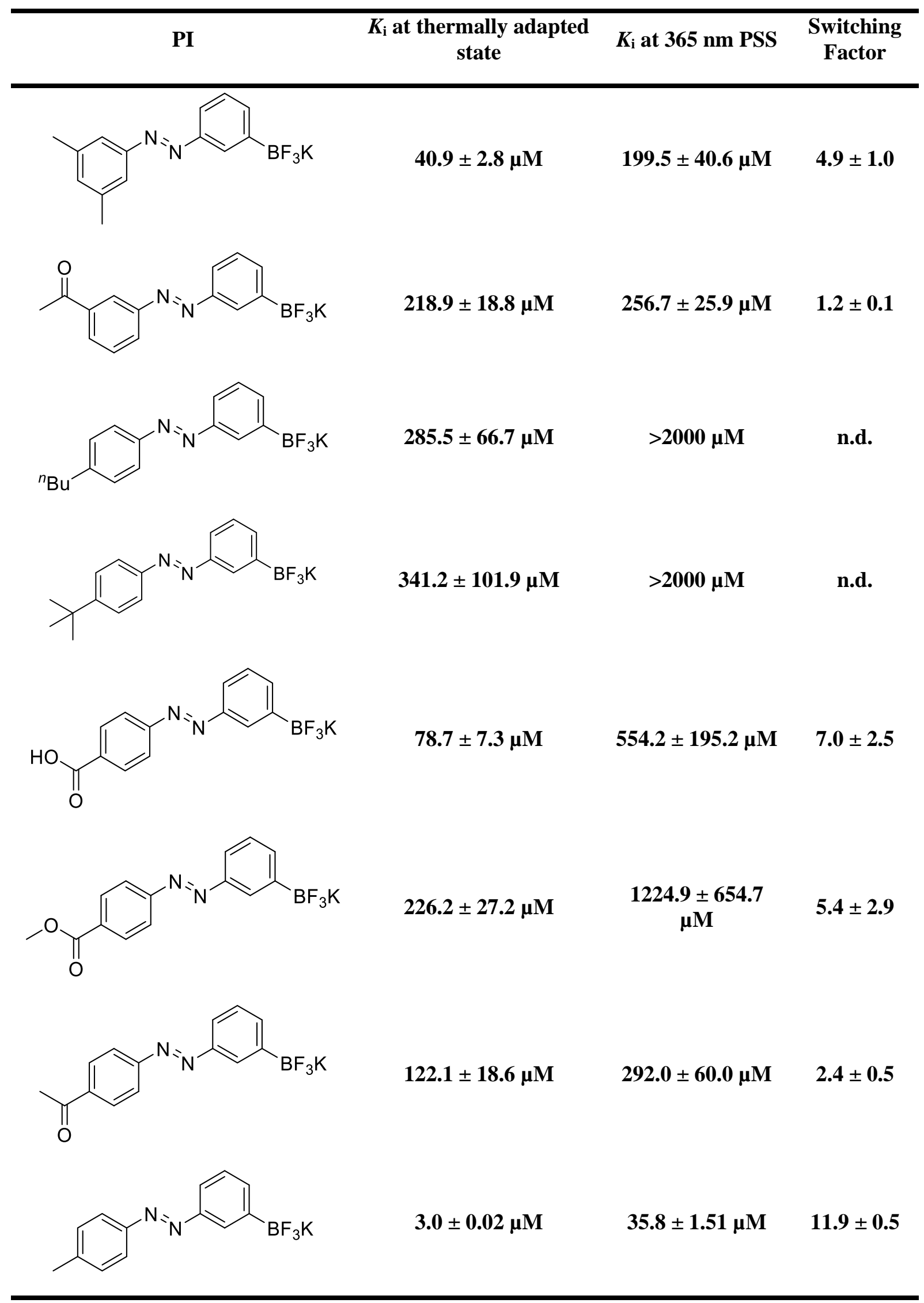




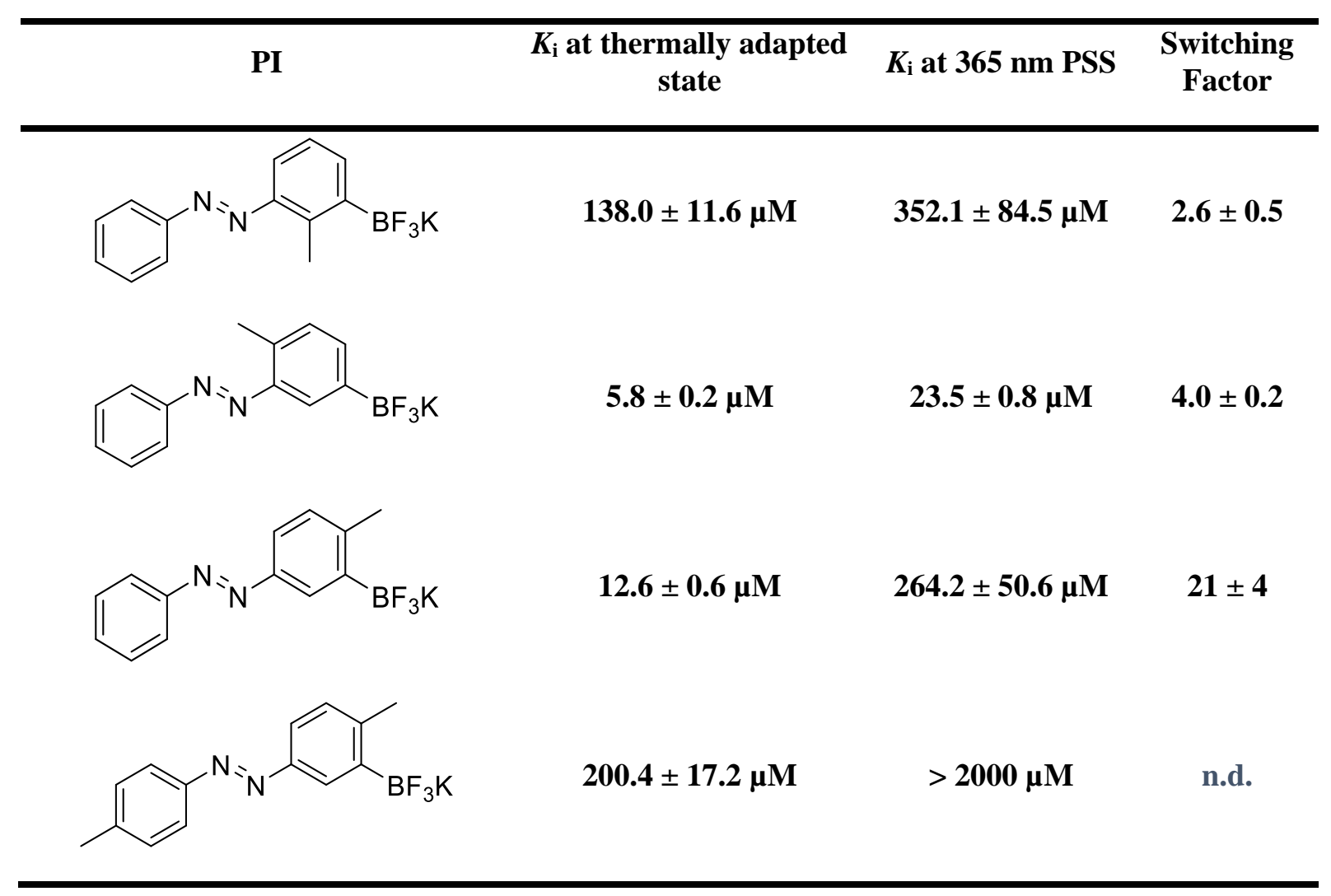




\subsubsection{Determination of Inhibition Constant $\left(K_{i}\right)$ of PI (20) on Ap}

Following the general procedure outlined in 4.4.1, the inhibition constants $\left(K_{\mathrm{i}}\right)$ of PI (20) on aminopeptidase M (Ap) at the thermally adapted and at the $365 \mathrm{~nm}$ photostationary state were determined. The Michaelis-Menten plots and the $K_{\mathrm{i}}$ values for the PI (20)/aminopeptidase M system are depicted below.

PI and results

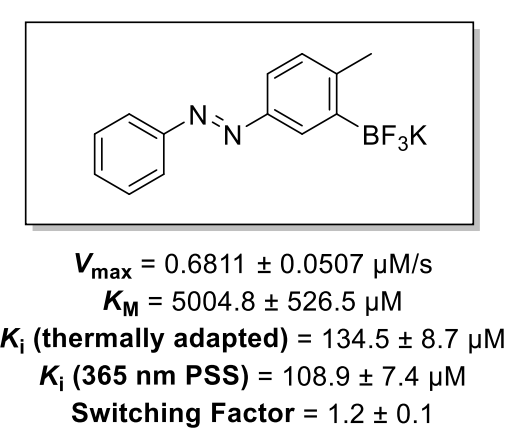

$50 \mu \mathrm{M}$ Cr-PI (thermally adapted)

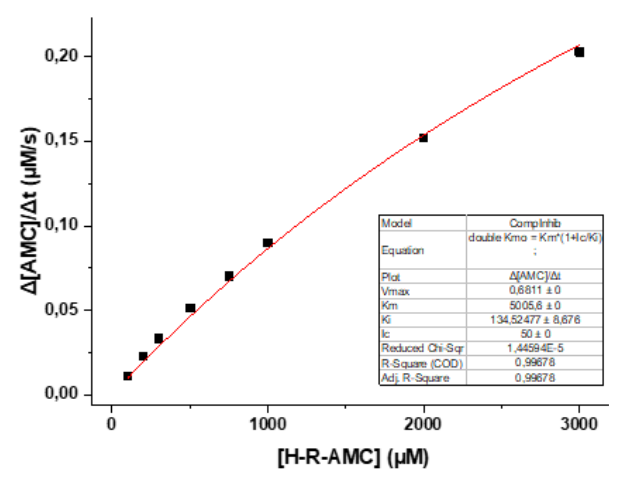

No Inhibitor

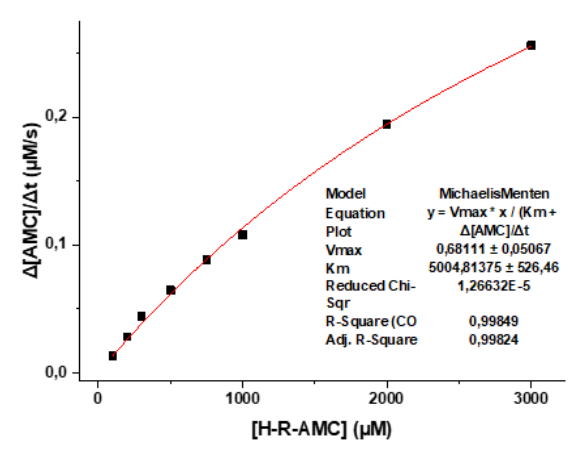

$50 \mu \mathrm{M}$ Cr-PI (365 nm PSS)

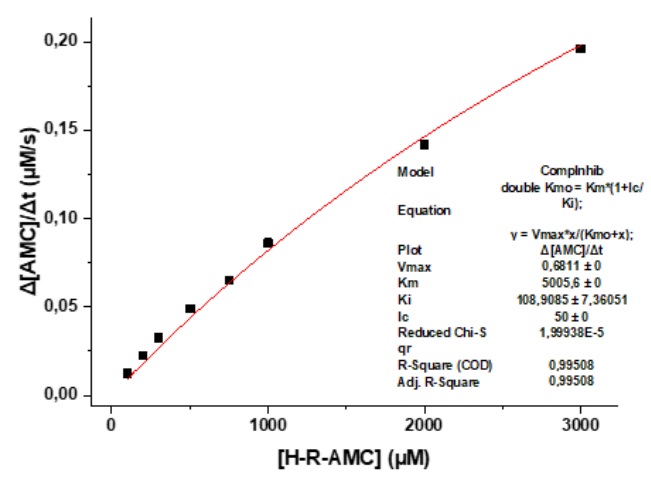

Figure S84. Michaelis-Menten plot and determined $K_{\mathrm{i}}$ values for the system PI (20)/aminopeptidase M at thermally adapted state and $365 \mathrm{~nm}$ PSS. 


\subsection{Batch Photoswitching (Main Text Fig. 2B)}

To investigate the reversibility of both the photoswitching process and the mode of inhibition, a batch photoswitching experiment using PI (20) was performed. In addition, this experiment allows to investigate the stability of the protease $\alpha$-chymotrypsin $(\mathrm{Cr})$ under prolonged times of 365 and $460 \mathrm{~nm}$ irradiation.

All required stock solutions were prepared as outlined below:

\section{Tris/Ca ${ }^{2+} / \mathrm{BSA}$ buffer $(200 \mathrm{mM} / 20 \mathrm{mM} / \mathbf{0 . 1} \mathrm{wt} \% ; \mathbf{p H}=\mathbf{7 . 8 1})($ Buffer $)$}

The Tris/ $\mathrm{Ca}^{2+}$ buffer $(200 \mathrm{mM} / 20 \mathrm{mM} ; \mathrm{pH}=7.81)$ was prepared by adding $50 \mathrm{~mL}$ of a $1 \mathrm{M}$ Tris solution and $2.5 \mathrm{~mL}$ of a $2 \mathrm{M} \mathrm{CaCl}_{2}$ solution to $197.5 \mathrm{~mL}$ of MilliQ. The $\mathrm{pH}$ was adjusted by the dropwise addition of $1 \mathrm{M} \mathrm{HCl}$. By adding $50 \mathrm{mg}$ of BSA to $50 \mathrm{~mL}$ of the Tris/ $/ \mathrm{Ca}^{2+}$ buffer $(200 \mathrm{mM} / 20 \mathrm{mM}$; $\mathrm{pH}=7.81)$, the Tris/ $\mathrm{Ca}^{2+} / \mathrm{BSA}$ buffer $(200 \mathrm{mM} / 20 \mathrm{mM} / 0.1 \mathrm{wt} \%$; $\mathrm{pH}=7.81)$ was obtained (BSA is added to prevent the adsorption of $\mathrm{Cr}$ onto plastic, e.g. pipette tips or tubing).

\section{Suc-Ala-Ala-Pro-Phe-AMC (400 $\boldsymbol{\mu M})$ Cr substrate solution (Cr-substrate)}

$26.6 \mu \mathrm{L}$ of a Suc-Ala-Ala-Pro-Phe-AMC stock solution (150 mM in DMSO) were diluted with $9973.3 \mu \mathrm{L}$ of Buffer to obtain $10 \mathrm{~mL}$ of a $400 \mu \mathrm{M}$ Suc-Ala-Ala-Pro-Phe-AMC Cr substrate solution.

\section{Chymotrypsin enzyme solution ( $0.2 \mu \mathrm{M}, \mathrm{Cr}$-enzyme)}

$200 \mu \mathrm{L}$ of chymotrypsin $\left(10 \mu \mathrm{M}\right.$ in $\left.2 \mathrm{mM} \mathrm{HCl} / 20 \mathrm{mM} \mathrm{Ca}^{2+}\right)$ were diluted with $9800 \mu \mathrm{L}$ of Buffer.

\section{Chymotrypsin photoswitchable inhibitor solution (1 mM, PI (20))}

$1.51 \mathrm{mg}$ of PI (20) were weighted out and dissolved in $5000 \mu \mathrm{L}$ of Buffer.

\section{Procedure}

1) $8 \mathrm{~mL}$ PI (20), $7 \mathrm{~mL}$ Buffer and $1 \mathrm{~mL}$ Cr-enzyme were mixed in a vial (Batch-Solution).

2) Three aliquots á $160 \mu \mathrm{L}$ were taken and added into a well of a 96-well plate. To each well, $40 \mu \mathrm{L}$ of Cr-substrate solution was added and the fluorescence was directly monitored using a Tecan Spark M10 plate reader. The slope (AMC production over time) was determined based on the obtained data.

3) The whole Batch-Solution was irradiated for $5 \mathrm{~min}$ with light of the $365 \mathrm{~nm}$ LED. Afterwards, three aliquots á $160 \mu \mathrm{L}$ were taken and added into a well of a 96-well plate. To each well, $40 \mu \mathrm{L}$ of Cr-substrate solution was added and the fluorescence was directly monitored using a Tecan Spark M10 plate reader. The slope (AMC production over time) was determined based on the obtained data.

4) Next, the whole Batch-Solution was irradiated for $5 \mathrm{~min}$ with light of the $460 \mathrm{~nm}$ LED. Afterwards, three aliquots á $160 \mu \mathrm{L}$ were taken and added into a well of a 96-well plate. To each well, $40 \mu \mathrm{L}$ of $\mathbf{C r}$-substrate solution was added and the fluorescence was directly monitored using a Tecan Spark M10 plate reader. The slope (AMC production over time) was determined based on the obtained data.

5) Steps $3 \& 4$ were repeated seven times.

The fluorescence intensity of wells within 96-well plates (purchased from Greiner Bio-One), containing $200 \mu \mathrm{L}$ of the respective solution, was monitored for $40 \mathrm{~s}$ (shaking 3s/orbital mode/amplitude $4 \mathrm{~mm}$ ) at 
$25{ }^{\circ} \mathrm{C}$ using top fluorescence reading mode $\left(\lambda_{\mathrm{ex}} / \lambda_{\mathrm{em}}=380 \mathrm{~nm} / 460 \mathrm{~nm}\right)$, as described in the General Information (see 1.1) part of this supplementary information.

\section{Results}

The respective irradiation status was plotted against the averaged difference of AMC production over time $(\Delta[\mathrm{AMC}] / \Delta \mathrm{t})$ of the three measurements. The results demonstrate that both the photoswitching and the mode of inhibition are reversible. In addition, the performance of the protease $\mathrm{Cr}$ is consistent even after a total of 40 minutes of 365 and $460 \mathrm{~nm}$ irradiation.

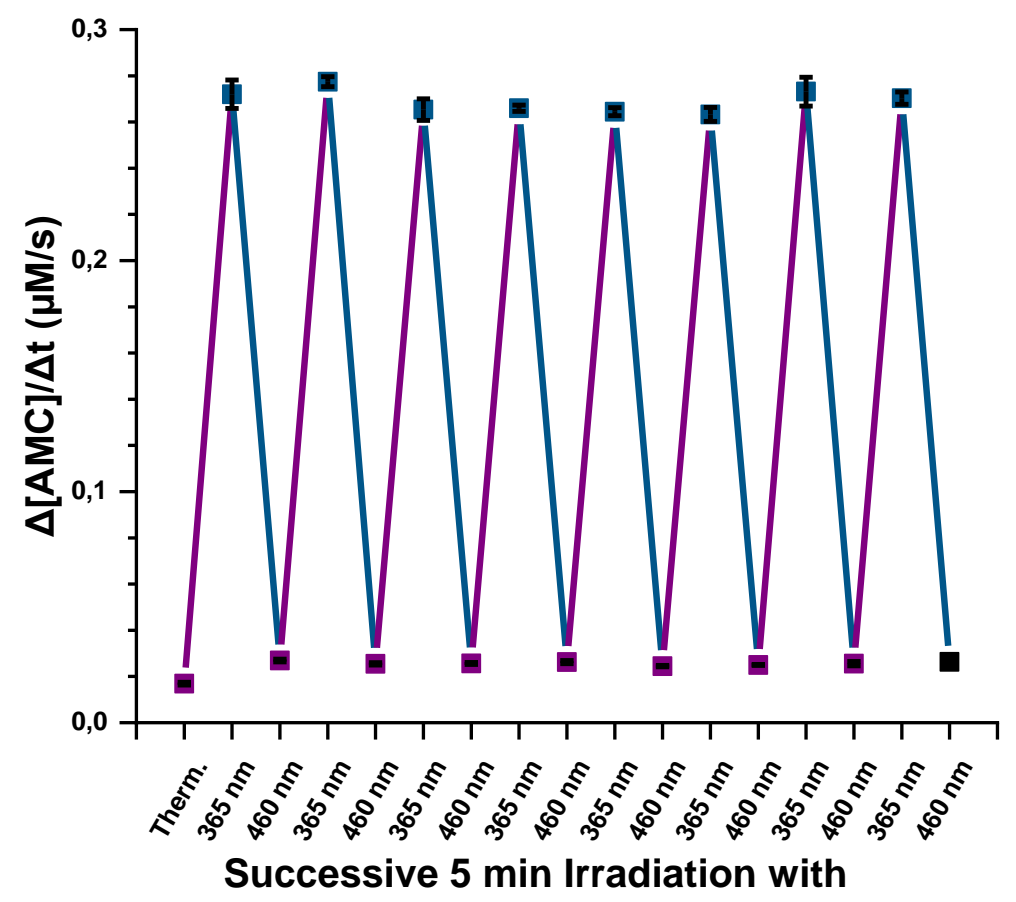

Figure S85. Results of the batch photoswitching experiment using PI (20). 


\subsection{Dose-Response-Curve}

To identify optimal flow conditions for later applications, we recorded a Dose-Response-Curve using the Suc-AAPF-AMC based fluorophoric assay by varying the concentration of PI (20) and measuring the initial slope of AMC formation over time for both its trans and the cis isomer.

All required stock solutions were prepared as outlined below:

Tris/Ca ${ }^{2+} / \mathrm{BSA}$ buffer $(200 \mathrm{mM} / 20 \mathrm{mM} / 0.1 \mathrm{wt} \% ; \mathrm{pH}=7.81)($ Buffer $)$

The Tris/ $\mathrm{Ca}^{2+}$ buffer $(200 \mathrm{mM} / 20 \mathrm{mM} ; \mathrm{pH}=7.81)$ was prepared by adding $50 \mathrm{~mL}$ of a $1 \mathrm{M}$ Tris solution and $2.5 \mathrm{~mL}$ of a $2 \mathrm{M} \mathrm{CaCl}_{2}$ solution to $197.5 \mathrm{~mL}$ of MilliQ. The $\mathrm{pH}$ was adjusted by the dropwise addition of $1 \mathrm{M} \mathrm{HCl}$. By adding $50 \mathrm{mg}$ of BSA to $50 \mathrm{~mL}$ of the Tris $/ \mathrm{Ca}^{2+}$ buffer $(200 \mathrm{mM} / 20 \mathrm{mM}$; $\mathrm{pH}=7.81)$, the Tris/ $\mathrm{Ca}^{2+} / \mathrm{BSA}$ buffer $(200 \mathrm{mM} / 20 \mathrm{mM} / 0.1 \mathrm{wt} \%$; $\mathrm{pH}=7.81)$ was obtained (BSA is added to prevent the adsorption of $\mathrm{Cr}$ onto plastic, e.g. pipette tips or tubing).

Suc-Ala-Ala-Pro-Phe-AMC (400 $\boldsymbol{\mu M})$ Cr substrate solution (Cr-substrate)

$26.6 \mu \mathrm{L}$ of a Suc-Ala-Ala-Pro-Phe-AMC stock solution (150 mM in DMSO) were diluted with 9973.3 $\mu \mathrm{L}$ of Buffer to obtain $10 \mathrm{~mL}$ of a $400 \mu \mathrm{M}$ Suc-Ala-Ala-Pro-Phe-AMC Cr substrate solution.

\section{Chymotrypsin enzyme solution ( $0.2 \mu \mathrm{M}$, Cr-enzyme)}

$200 \mu \mathrm{L}$ of chymotrypsin $\left(10 \mu \mathrm{M}\right.$ in $\left.2 \mathrm{mM} \mathrm{HCl} / 20 \mathrm{mM} \mathrm{Ca}^{2+}\right)$ were diluted with $9800 \mu \mathrm{L}$ of Buffer.

\section{Chymotrypsin photoswitchable inhibitor solution (1 mM, PI (20))}

$3.02 \mathrm{mg}$ of PI 20 were weighted out and dissolved in $10 \mathrm{~mL}$ of Buffer.

\section{Procedure}

Fluorogenic assays were performed at fixed enzyme and substrate concentration in the presence of varying PI concentrations according to the pipetting scheme outlined in Table S4.

Table S4. Pipetting scheme for the Dose-Response-Curve of Cr and PI (20). All experiments were done as triplicates using the autoinjector of the Tecan SparkM10 plate reader for the addition of the Cr-enzyme to each well prior to the respective measurement.

\begin{tabular}{|c|c|c|c|c|c|c|c|}
\hline $\begin{array}{c}{[\text { Cr-substrate }]_{\text {well }} /} \\
\mu \mathrm{M}\end{array}$ & $\begin{array}{c}\mathrm{V} \text { (Cr-substrate, } 400 \\
\mu \mathrm{M} \text { stock }) / \mu \mathrm{L}\end{array}$ & $\begin{array}{c}{[\text { Cr-enzyme }]_{\text {well }} /} \\
\mu \mathrm{M}\end{array}$ & $\begin{array}{c}\mathrm{V}(\mathrm{Cr} \text {-enzyme, } 0.2 \\
\mu \mathrm{M} \text { stock }) / \mu \mathrm{L}\end{array}$ & {$[\mathrm{PI}]_{\mathrm{well}} / \boldsymbol{\mu M}$} & $\begin{array}{c}\mathrm{V} \text { (PI, } 1 \mathrm{mM} \text { stock) / } \\
\mu \mathrm{L}\end{array}$ & $\begin{array}{c}\text { Buffer or Buffer- } \\
\text { DMSO } / \mu \mathrm{L}\end{array}$ & Total Volume / $\mu \mathrm{L}$ \\
\hline 80 & 40 & 0.01 & 10 & 0 & 0 & 150 & 200 \\
\hline 80 & 40 & 0.01 & 10 & 25 & 5 & 145 & 200 \\
\hline 80 & 40 & 0.01 & 10 & 50 & 10 & 140 & 200 \\
\hline 80 & 40 & 0.01 & 10 & 100 & 20 & 130 & 200 \\
\hline 80 & 40 & 0.01 & 10 & 150 & 30 & 120 & 200 \\
\hline 80 & 40 & 0.01 & 10 & 200 & 40 & 110 & 200 \\
\hline 80 & 40 & 0.01 & 10 & 250 & 50 & 100 & 200 \\
\hline 80 & 40 & 0.01 & 10 & 300 & 60 & 90 & 200 \\
\hline 80 & 40 & 0.01 & 10 & 400 & 80 & 70 & 200 \\
\hline 80 & 40 & 0.01 & 10 & 500 & 100 & 50 & 200 \\
\hline 80 & 40 & 0.01 & 10 & 750 & 150 & 0 & 200 \\
\hline
\end{tabular}

All compounds except chymotrypsin have been pipetted using multichannel pipettes. The enzyme chymotrypsin was then added to the respective well of the 96 well-plate using the Tecan SparkM10 autoinjector. All experiments were done as triplicates. The fluorescence intensity of wells within 96well plates (purchased from Greiner Bio-One), containing $200 \mu \mathrm{L}$ of the respective solution, was monitored for $40 \mathrm{~s}$ (shaking 3s/orbital mode/amplitude $4 \mathrm{~mm}$ ) at $25^{\circ} \mathrm{C}$ using top fluorescence reading mode $\left(\lambda_{\mathrm{ex}} / \lambda_{\mathrm{em}}=380 \mathrm{~nm} / 460 \mathrm{~nm}\right)$, as described in the General Information (see 1.1) part of this supplementary information. Once the curve for the thermally-adapted trans-isomer has been determined, $2 \mathrm{~mL}$ of the same PI stock solution were irradiated with UV-A light from a LED $\left(30 \mathrm{~W}, \lambda_{\max }=370 \mathrm{~nm}\right)$ 
for $10 \mathrm{~min}$ to reach the $370 \mathrm{~nm}$ photostationary state and the assay was repeated. In both cases, the amount of fluorescent product was calculated according to the AMC calibration curve (see 4.2). The experimental data obtained was the fitted using the Origin $2018 \mathrm{~b}$ software package.

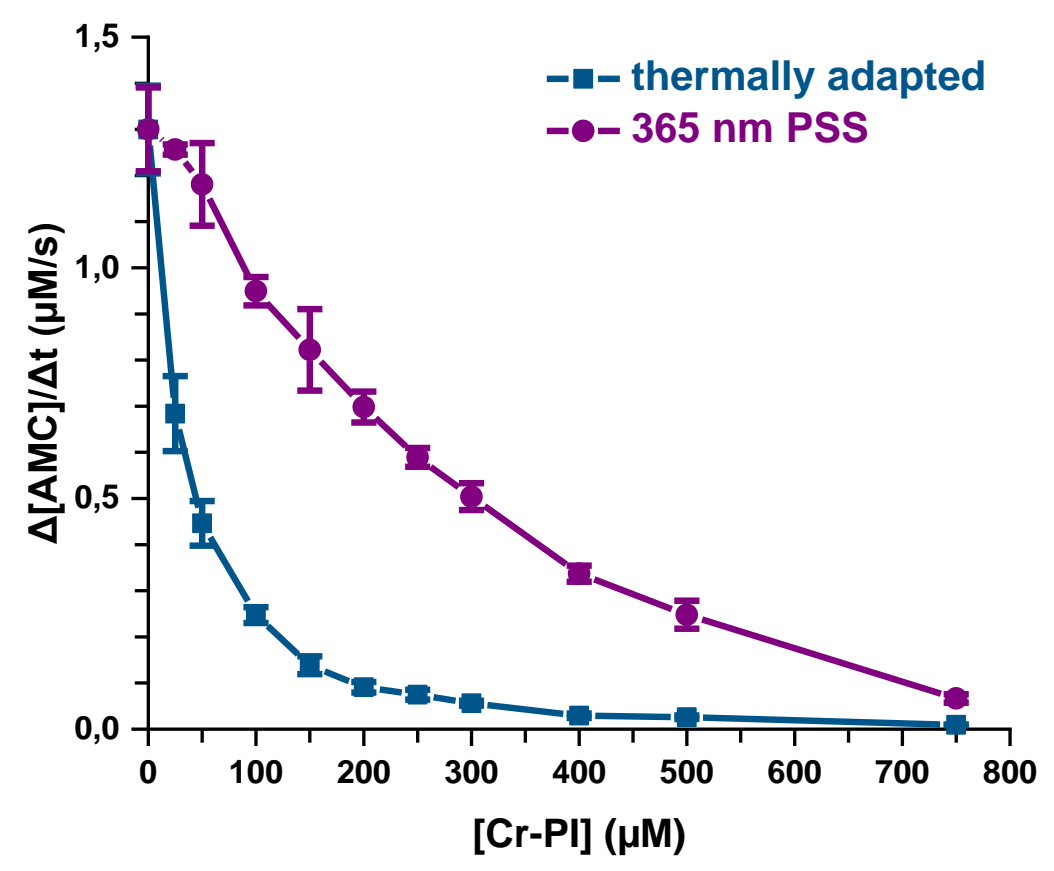

Figure S86. Results of the Dose-Response-Curve of Cr and PI (20). 


\section{Flow-experiments using a Continuous-Stirred Flow Tank Reactor}

\subsection{General Information and Procedures}

\subsubsection{Preparation of Continuous-Stirred Flow Tank Reactor (CSTR)}

Continuous-Stirred Flow Tank Reactors (CSTRs) were prepared from a PDMS reactor vessel which encases tubing and glass slide. The PDMS reaction vessel is prepared by covering up the height of a brass cylinder (typically with a volume of $216 \mu \mathrm{L}$ ) plus $1.5 \mathrm{~cm}$ in a petri dish with a mixture of $10 \mathrm{wt} \%$ Sylgard ${ }^{\circledR} 184$ silicone elastomer curing agend crosslinker in Sylgard ${ }^{\circledR} 184$ silicone elastomer base. Air bubbles are removed from the elastomer mixture by degassin the mixture carefully for $1 \mathrm{~h}$ in a vacuum dessicator. The elastomer is then cured in a $65^{\circ} \mathrm{C}$ oven for $2 \mathrm{~h}$. After polymerization, the PDMS reactor vessel is removed from the oven. Four inflow holes are punched in a square pattern into the side of the PDMS reactor using a biopsy punch. The brass cylinder is removed from the reactor vessel to leave a cavity for the reactor chamber and an outflow hole is punched into the reactor vessel. The reactor vessel piece (with an inserted micro stirrer bar) is then bound to a glass slide (previously cleaned with isopropanol and dried under nitrogen) via oxygen plasma treatment. The reactor is afterwards placed in a $100{ }^{\circ} \mathrm{C}$ overnight oven to completely set. After removal and cooling Teflon tubing with appropriate inner diameters $(0.38 \mathrm{~mm}$ for inlets, $0.56 \mathrm{~mm}$ for outlet) is inserted into the punched holes. The outflow tubing is sealed in position with a small amount of elastomer mixture at the joint between the tubing and the reactor. The seal is cured at $65^{\circ} \mathrm{C}$ for $2 \mathrm{~h}$. A typical CSTR has a volume of $196 \mu \mathrm{L}$ (Volume of reaction cavity - Volume of micro stirrer bar $=216 \mu \mathrm{L}-20 \mu \mathrm{L}$ ). Continuous-stirred Flow Tank Reactors with filter inlet were constructed in a similar manner than described above for the performance of flow experiments with irradiation of the reactor.

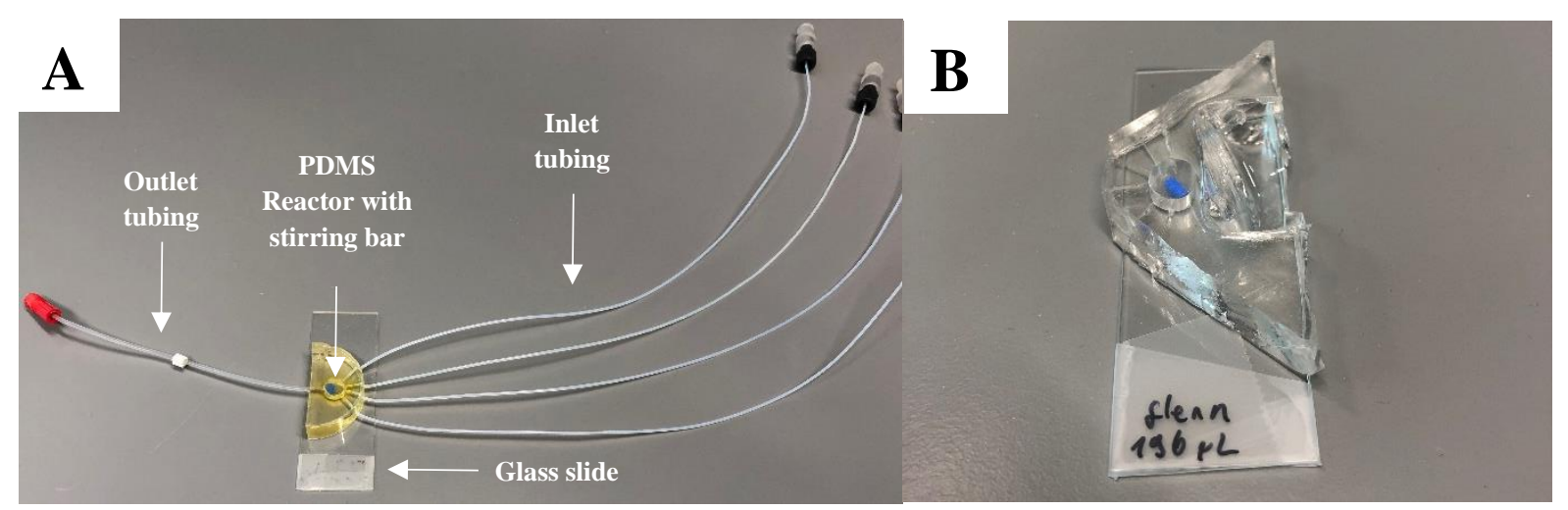

Figure S87. A) Picture of a Continuous-Stirred Flow Tank Reactor used for flow experiments. It has four inlets (black connectors) and one outlet (red connector). B) Continuous-Stirred Flow Tank Reactor with filter inlet constructed for the performance of flow experiments with irradiation of the reactor. The filter is placed inside the cavity, thereby ensuring a reproducible irradiation of the reactor throughout experiments. In case of additional filters being used, similar reactors with two filter inlets were constructed or the respective LED was fixed using a tripod/holder setup. 


\subsubsection{Overview of the Setup Used for Flow Experiments}

The setup of a typical continuous-stirred tank reactor (CSTR) experiment is depicted in Fig. S86. The CSTR is placed upon a magnetic stirrer plate and reactant solutions (e.g. chymotrypsin, PI etc.) are flowed in from glass syringes attached to a NEMESYS pump system that allows precise regulation of the respective flow rates. The outflow of the CSTR is connected to a microfluidics T-junction where the solution is mixed with the respective Cr-substrate. The outflow of the microfluidics T-junction is connected to a lab-made flow-cuvette (see Fig. S1) placed into an Avantes spectrofluorophotometer (see 1.1), which is controlled and the data recorded with the AvaSpec14.1 software package. The fluorescence intensity was continuously measured and saved every 15 seconds in an Excel file. The outflow of the flow cuvette is treated as waste and thus collected in a beaker. In case of reactor irradiation, the mounted UV LED is placed in front of PDMS reactor with a filter placed inside the filter inlet and fixed with clamps. The blue LED (or any additional LEDs) is placed at the top or around the reactor to ensure sufficient irradiation and is being fixed with clamps. All LEDs are powered by Thorlabs LEDD1B drivers connected to a Thorlabs KCH601 controller unit. While for some experiments the respective LED was manually regulated in terms of intensity and "on" or "off" state, the LEDs were controlled by the computer in case of kinetic filtering or pulse delay experiments. Therefore, the LEDD1B drivers were connected to an ELEGGO UNO R3 controller unit which is connected to a notebook. A Python code for precisely controlling the LED output was written which allows the precise regulation of the LED's intensity or the timing of irradiation using an Arduino setup (see 6). The spectrophotometer and the flow cuvette are protected from light (e.g. from the used LEDs) and thus placed inside a black box. Additionally, both outlet and inlet tubing of the reactor were covered with alumina foil to prevent irradiation of the solutions prior entering or after flushing out of the reactor. 

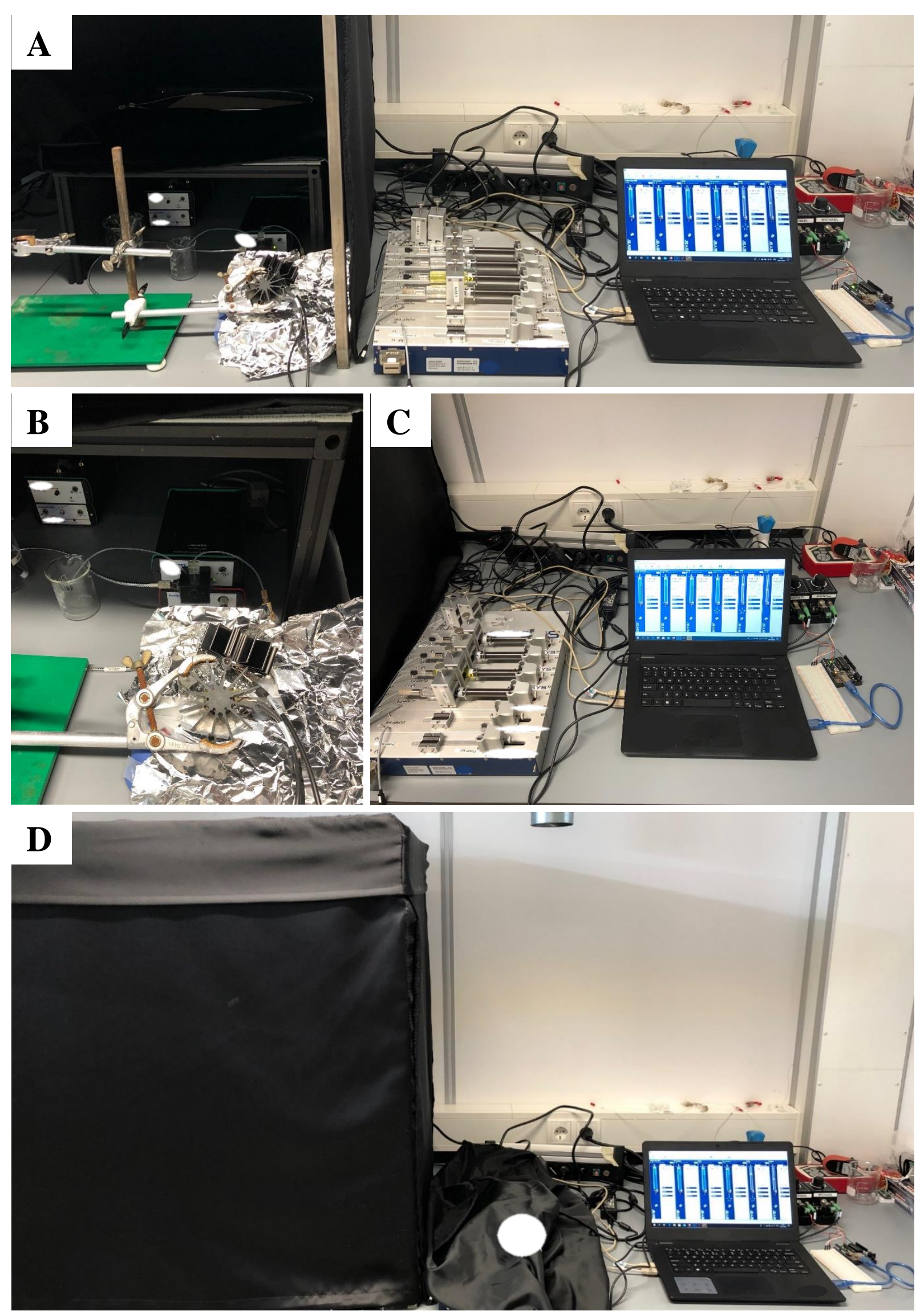

Figure S86. A) Complete overview of the used setup with computer, flow dosing units, LED controller, CSTR on a stirrer plate with surrounding LEDs and filter and fluorescence spectrophotometer. B) CSTR reactor on the stirrer plate with two LEDs focused at the reactor. C) Flow Dosing Units, Computer, LED controller and Arduino setup to regulate the irradiation duration, intensity and source. D) Overview of the whole setup throughout the performance of an experiment. All reagents were covered from light to prevent any potential decomposition of the reagents. 


\subsubsection{Preparation of Solutions for Flow Experiments}

All reactants were dissolved in specific buffers whose composition and concentration are depicted in Table S5. These buffer concentrations were used for all experiments unless otherwise specified in the next sections and the volumes per experiment are also given in the next sections. All solutions were made with MilliQ water. For stability purposes, calcium chloride was added to Chymotrypsin solutions and magnesium chloride to aminopeptidase solutions. Bovine serum albumin (BSA) has been added to all buffers to prevent adsorption of the reagents to the tubing or PDMS reactor.

Table S5. Buffer solutions used in all of the flow experiments unless otherwise stated.

\begin{tabular}{ll}
\hline Solutions & Buffer Composition \\
\hline Cr buffer & $200 \mathrm{mM}$ Tris, $20 \mathrm{mM} \mathrm{Ca}^{2+}$ and $0.1 \mathrm{wt} \% \mathrm{BSA}$, \\
& $\mathrm{pH}=7.8$ \\
PI & $200 \mathrm{mM}$ Tris, $20 \mathrm{mM} \mathrm{Ca}^{2+}$ and $0.1 \mathrm{wt} \% \mathrm{BSA}$, \\
& $\mathrm{pH}=7.8$ \\
Cr-substrate (Suc-Ala-Ala-Pro-Phe-AMC) & $200 \mathrm{mM}$ Tris, $20 \mathrm{mM} \mathrm{Ca}^{2+}$ and $0.1 \mathrm{wt} \% \mathrm{BSA}$, \\
& $\mathrm{pH}=7.8$ \\
Z-Phe-Arg-AMC & $200 \mathrm{mM}$ Tris, $20 \mathrm{mM} \mathrm{Ca}^{2+}, 10 \mathrm{mM} \mathrm{Mg}^{2+}$ and \\
& $0.1 \mathrm{wt} \% \mathrm{BSA}, \mathrm{pH}=7.8 ; 10 \mathrm{vol}-\% \mathrm{DMSO}^{2}$ added \\
Flow experiment buffer & $200 \mathrm{mM}$ Tris, $20 \mathrm{mM} \mathrm{Ca}^{2+}$ and $0.1 \mathrm{wt}^{2} \mathrm{BSA}$, \\
& $\mathrm{pH}=7.8$ \\
Ap buffer & $200 \mathrm{mM}$ Tris, $20 \mathrm{mM} \mathrm{Ca}^{2+}, 10 \mathrm{mM} \mathrm{Mg}^{2+}$ and \\
& $0.1 \mathrm{wt} \% \mathrm{BSA}, \mathrm{pH}=7.8$ \\
\hline
\end{tabular}




\subsubsection{Initiating the Flow Experiment}

In order to set up the CSTR, the inflow tubing for each solution is filled until the solution is just about to enter the reactor cavity. To fill the reactor cavity a total flowrate of $16000 \mu \mathrm{L} \mathrm{h}^{-1}$ is used, with the individual flow rates in the same ratio as used in the experiment itself. A flow rate of $16000 \mu \mathrm{L} \mathrm{h}^{-1}$ is maintained until all air is removed from the CSTR and the outflow tubing is filled. Then the flow rates of the respective reagents are lowered to that required for the experiment. The outflow tubing is connected to the flow cuvette via a microfluidics $\mathrm{T}$-junction into which in most experiments the respective Cr-substrate is added. If no substrate is added, one inlet of the microfluidics T-junction is sealed. The AMC concentration (illustrating the activity of the respective enzyme or the amount of formed fluorescent product) is determined by fluorescence measurement.

\subsubsection{Conversion of Fluorescence Intensity to AMC Concentration}

A calibration for the conversion of fluorescence intensity to the concentration of 7-amino-4methylcoumarin (AMC, $100 \mu \mathrm{M}$ ) was performed prior and after each performed experiment. A stock solution of 7-amino-4-methylcoumarin (AMC) in a buffer (200 mM Tris, $20 \mathrm{mM} \mathrm{Ca}^{2+}$ and $0.1 \mathrm{wt} \%$ $\mathrm{BSA}, \mathrm{pH}=7.8$ ) was prepared freshly for each respective experiment. By diluting the AMC stock solution with different flow rates of a buffer solution $\left(200 \mathrm{mM}\right.$ Tris, $20 \mathrm{mM} \mathrm{Ca}^{2+}$ and $0.1 \mathrm{wt} \% \mathrm{BSA}$, $\mathrm{pH}=7.8$ ) using our flow setup, different AMC concentration result at the flow cuvette for fluorescence analysis. The respective dilution scheme is outlined in Table S6.

Table S6. Dilution scheme for conversion of fluorescence intensity to AMC concentration.

\begin{tabular}{ccc}
\hline Flowrate AMC / $(\boldsymbol{\mu L} / \mathbf{h})$ & Flowrate buffer $/(\boldsymbol{\mu L} / \mathbf{h})$ & {$[\mathbf{A M C}] / \boldsymbol{M M}$} \\
\hline 5000 & 95000 & 5 \\
5000 & 45000 & 10 \\
5000 & 25000 & 16.6 \\
5000 & 15000 & 25 \\
5000 & 10000 & 33.3 \\
5000 & 5000 & 50 \\
5000 & 2500 & 66.6 \\
7500 & 2500 & 75 \\
10000 & $/$ & 100 \\
\hline
\end{tabular}

Due to the use of different flow cuvettes or due to exchange of other parts of the flow setup, e.g. the CSTR or the pump dosing units, we performed this external calibration prior to the performance of each experiment, since we observed significant changes upon replacing some parts. Additionally, to further proof the correct conversion of measured fluorescence intensity to AMC concentration, we performed the same calibration at the end of the respective experiment. In all cases, no significant differences in between both external calibrations were observed (maximum change $~ 2 \%$ between both calibrations). This further demonstrates the robustness and reproducibility of our developed setup. A representative example of the results of an external calibration is outlined in Fig. S88. 


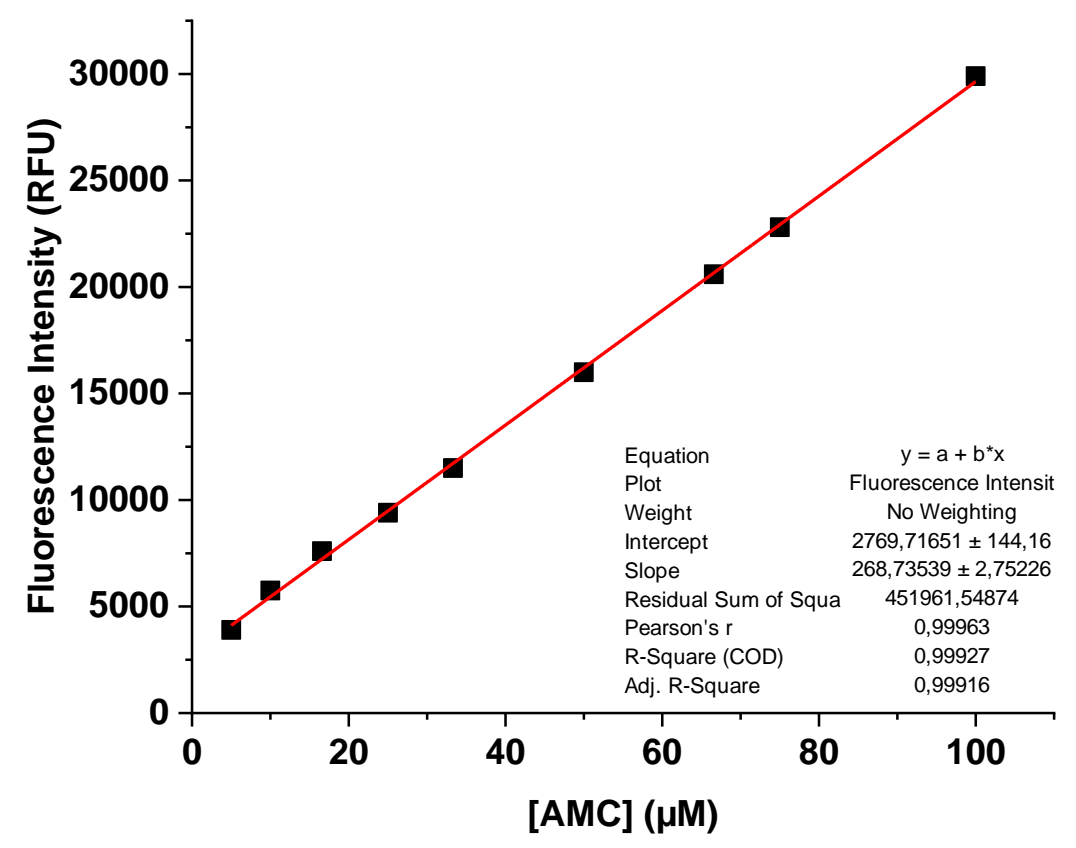

Figure S88. Representative example of an external "fluorescence intensity/AMC concentration" calibration plot with linear fitting of the data.

\subsubsection{Data Processing}

All data was processed using Origin 2018b. The fluorescence intensity was converted to AMC concentration based on the respective external calibrations, as outlined in 5.1.5. Unless otherwise noted, no further data processing (e.g. smoothing of multiple data points) was performed. 


\subsection{Experimental Details and Results}

\subsubsection{Multi-State Activity Control (Main Text Fig. 3C)}

The flow experiment was performed using a self-made CSTR with an inner volume of $196 \mu \mathrm{L}$ (stirring bar taken into account). Throughout the whole experiment, the overall flow rate was kept at $1200 \mu \mathrm{L} / \mathrm{h}$, resulting in a $k_{\text {flow }}$ of $6.1 \mathrm{~h}^{-1}$. The experimental conditions (concentration of compound in feed syringe, flowrate and final concentration of the respective compound in flow cuvette) for the flow experiment are outlined in Table $\mathrm{S} 7$.

Table S7. Experimental conditions for the flow experiment: concentration of compound in feed syringe, flowrate and final concentration of the respective compound in flow cuvette.

\begin{tabular}{cccc}
\hline Compound & $\begin{array}{c}\text { Flowrate } / \\
(\boldsymbol{\mu L} / \mathbf{h})\end{array}$ & $\begin{array}{c}\text { Concentration in Feed Syringe } \\
/ \boldsymbol{\mu M}\end{array}$ & $\begin{array}{c}\text { Concentration in } \\
\text { Flow Cuvette } / \boldsymbol{\mu M}\end{array}$ \\
\hline Cr & 400 & $0.12 \mu \mathrm{M}$ & $0.04 \mu \mathrm{M}$ \\
PI (20) & 400 & $600 \mu \mathrm{M}$ & $200 \mu \mathrm{M}$ \\
Suc-AAPF-AMC & 200 & $480 \mu \mathrm{M}$ & $80 \mu \mathrm{M}$ \\
Buffer Syringe 1 & 100 & $200 \mathrm{mM} \mathrm{Tris,} 20 \mathrm{mM} \mathrm{Ca}^{2+}$ and \\
Buffer Syringe 2 & \multirow{4}{*}{$0.1 \mathrm{wt} \% \mathrm{BSA}, \mathrm{pH}=7.8$} \\
\end{tabular}

The light pulse sequence (light source, intensity, timing and duration) applied throughout the experiment is outlined in Table S8. For $365 \mathrm{~nm}$ irradiation, a Thorlabs bandpass filter was placed in between the CSTR and the LED. The power of light with respect to the stated intensity (e.g. 10, 20, 25, 40, 60, 80, $100 \%$ ) was measured using a Thorlabs PM100A power meter equipped with a photodiode sensor.

Table S8. Light pulse sequence applied throughout the performance of the flow experiment.

\begin{tabular}{|c|c|c|c|}
\hline Time & Light Source & Duration & Intensity \\
\hline Start & I & I & l \\
\hline $0.25 \mathrm{~h}$ & $365 \mathrm{~nm}$ Avonec & Continuous at $20 \%$ intensity & $\begin{array}{c}20 \% \mathrm{UV}= \\
10.5 \mathrm{~mW} / \mathrm{cm}^{2}\end{array}$ \\
\hline $1.5 \mathrm{~h}$ & $365 \mathrm{~nm}$ Avonec & $\begin{array}{l}20 \text { seconds pulse of } 100 \% \text { intensity, then } \\
\text { back to continuous } 20 \% \text {. }\end{array}$ & $\begin{array}{c}100 \% \mathrm{UV}= \\
35.6 \mathrm{~mW} / \mathrm{cm}^{2}\end{array}$ \\
\hline $2.22 \mathrm{~h}$ & $\begin{array}{c}365 \mathrm{~nm} \& 460 \mathrm{~nm} \\
\text { Avonec }\end{array}$ & $\begin{array}{l}\text { (1) UV off, then } 2 \text { min blue (100\%). (2) } \\
100 \% \text { UV for } 10 \text { min. (3) } 5 \text { min blue } \\
\text { (100\%). (4) continuous } 20 \% \text { UV. }\end{array}$ & $\begin{array}{c}100 \% \mathrm{UV}=35.6 \\
\mathrm{~mW} / \mathrm{cm}^{2} ; 100 \% \text { blue }= \\
133.6 \mathrm{~mW} / \mathrm{cm}^{2}\end{array}$ \\
\hline $3.1 \mathrm{~h}$ & $365 \mathrm{~nm}$ Avonec & $\begin{array}{c}45 \sec 100 \% \mathrm{UV} \text {, then back to continuous } \\
20 \% \mathrm{UV}\end{array}$ & $\begin{array}{c}100 \% \mathrm{UV}= \\
35.6 \mathrm{~mW} / \mathrm{cm}^{2}\end{array}$ \\
\hline $3.6 \mathrm{~h}$ & $365 \mathrm{~nm}$ Avonec & $\begin{array}{c}10 \sec 100 \% \text { UV, then back to continuous } \\
20 \% \text { UV }\end{array}$ & $\begin{array}{c}100 \% \mathrm{UV}= \\
35.6 \mathrm{~mW} / \mathrm{cm}^{2}\end{array}$ \\
\hline
\end{tabular}


The time/AMC concentration plot of this experiment, performed under out-of-equilibrium conditions using the previously described flow setup, is outlined in Fig. 89.

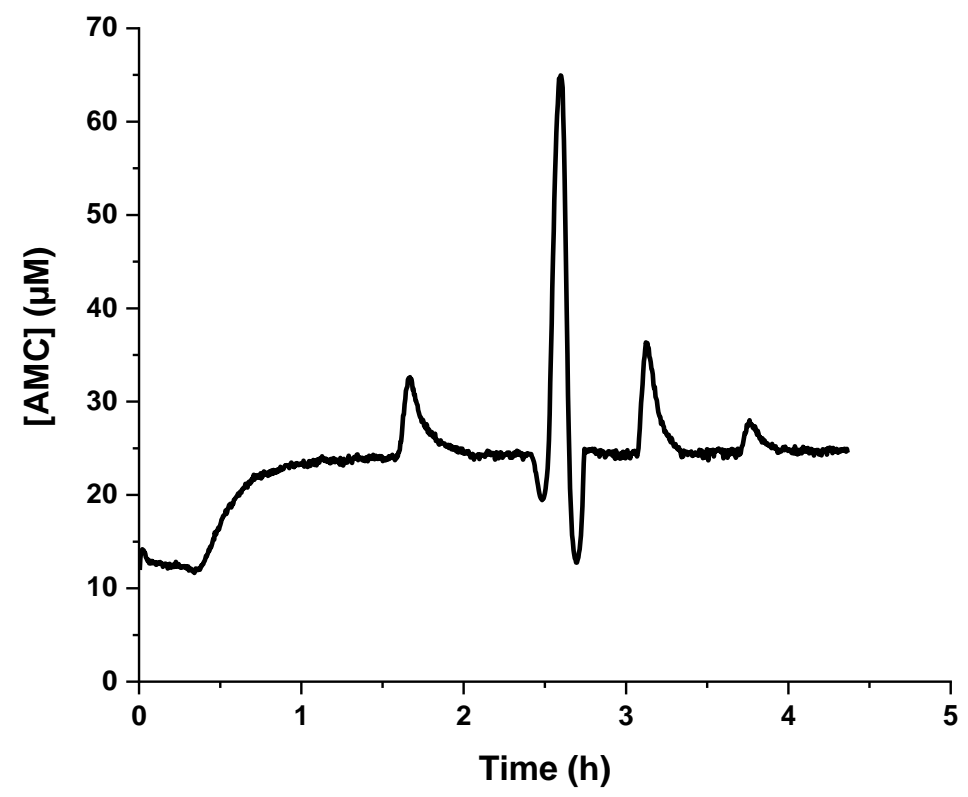

Figure S89. Results of the flow experiment: Generation of an electrocardiogram heartbeat AMC-output pattern. The above outlined conditions and light pulse sequences were applied throughout the experiment. 


\subsubsection{Alteration of Pulse Duration (Main Text Fig. 4A)}

\section{Experimental Details and Results: Pulse Sequence no blue irradiation}

The flow experiment was performed using a self-made CSTR with an inner volume of $196 \mu \mathrm{L}$ (stirring bar taken into account). Throughout the whole experiment, the overall flow rate was kept at $1200 \mu \mathrm{L} / \mathrm{h}$, resulting in a $k_{\text {flow }}$ of $6.1 \mathrm{~h}^{-1}$. The experimental conditions (concentration of compound in feed syringe, flowrate and final concentration of the respective compound in flow cuvette) for the flow experiment are outlined in Table S9.

Table S9. Experimental conditions for the flow experiment: concentration of compound in feed syringe, flowrate and final concentration of the respective compound in flow cuvette.

\begin{tabular}{cccc}
\hline Compound & $\begin{array}{c}\text { Flowrate } \\
(\boldsymbol{\mu L} / \mathbf{h})\end{array}$ & $\begin{array}{c}\text { Concentration in Feed Syringe } \\
/ \boldsymbol{\mu M}\end{array}$ & $\begin{array}{c}\text { Concentration in } \\
\text { Flow Cuvette } / \boldsymbol{\mu M}\end{array}$ \\
\hline Cr & 400 & $0.12 \mu \mathrm{M}$ & $0.04 \mu \mathrm{M}$ \\
PI (20) & 400 & $600 \mu \mathrm{M}$ & $200 \mu \mathrm{M}$ \\
Suc-AAPF-AMC & 200 & $480 \mu \mathrm{M}$ & $80 \mu \mathrm{M}$ \\
Buffer Syringe 1 & 100 & $200 \mathrm{mM} \mathrm{Tris,} 20 \mathrm{mM} \mathrm{Ca}^{2+}$ and \\
Buffer Syringe 2 & \multirow{4}{*}{$0.1 \mathrm{wt} \% \mathrm{BSA}, \mathrm{pH}=7.8$} \\
\hline
\end{tabular}

The light pulse sequence (light source, intensity, timing and duration) applied throughout the experiment is outlined in Table S10. For $365 \mathrm{~nm}$ irradiation, a Thorlabs bandpass filter was placed in between the CSTR and the LED. The power of light $\left(100 \%(365 \mathrm{~nm})=37.1 \mathrm{~mW} / \mathrm{cm}^{2}\right)$ was measured using a Thorlabs PM100A power meter equipped with a photodiode sensor. 
Table S10. Light pulse sequence applied throughout the performance of the flow experiment.

\begin{tabular}{cccc}
\hline Time & Light Source & Duration & Intensity \\
\hline Start & $/$ & $/$ & $/$ \\
$0.33 \mathrm{~h}$ & $365 \mathrm{~nm}$ Avonec & $10 \mathrm{~min}$ at 100\% intensity, then off & $100 \%$ \\
$1.33 \mathrm{~h}$ & $365 \mathrm{~nm}$ Avonec & $7.5 \mathrm{~min}$ at 100\% intensity, then off & $100 \%$ \\
$2.25 \mathrm{~h}$ & $365 \mathrm{~nm}$ Avonec & $5 \mathrm{~min}$ at 100\% intensity, then off & $100 \%$ \\
$3.10 \mathrm{~h}$ & $365 \mathrm{~nm}$ Avonec & 4 min at 100\% intensity, then off & $100 \%$ \\
$3.90 \mathrm{~h}$ & $365 \mathrm{~nm}$ Avonec & $3 \mathrm{~min}$ at 100\% intensity, then off & $100 \%$ \\
$4.70 \mathrm{~h}$ & $365 \mathrm{~nm}$ Avonec & $2 \mathrm{~min}$ at 100\% intensity, then off & $100 \%$ \\
$5.5 \mathrm{~h}$ & $365 \mathrm{~nm}$ Avonec & $1 \mathrm{~min}$ at 100\% intensity, then off & $100 \%$ \\
$6.3 \mathrm{~h}$ & $365 \mathrm{~nm}$ Avonec & $30 \mathrm{sec}$ at 100\% intensity, then off & $100 \%$ \\
$7.15 \mathrm{~h}$ & $365 \mathrm{~nm}$ Avonec & $15 \mathrm{sec}$ at 100\% intensity, then off & $100 \%$ \\
$7.85 \mathrm{~h}$ & $365 \mathrm{~nm}$ Avonec & $10 \mathrm{sec}$ at 100\% intensity, then off & $100 \%$ \\
$8.48 \mathrm{~h}$ & $365 \mathrm{~nm}$ Avonec & $5 \mathrm{sec}$ at 100\% intensity, then off & $100 \%$ \\
$8.93 \mathrm{~h}$ & $365 \mathrm{~nm}$ Avonec & $2 \mathrm{sec}$ at 100\% intensity, then off & $100 \%$ \\
$9.21 \mathrm{~h}$ & $365 \mathrm{~nm}$ Avonec & $1 \mathrm{sec}$ at 100\% intensity, then off & $100 \%$ \\
$9.50 \mathrm{~h}$ & $365 \mathrm{~nm}$ Avonec & $30 \mathrm{~min}$ at 100\% intensity, then off & $100 \%$ \\
\hline
\end{tabular}

The time/AMC concentration plot of this experiment, performed under out-of-equilibrium conditions using the previously described flow setup, is outlined in Figure S90.

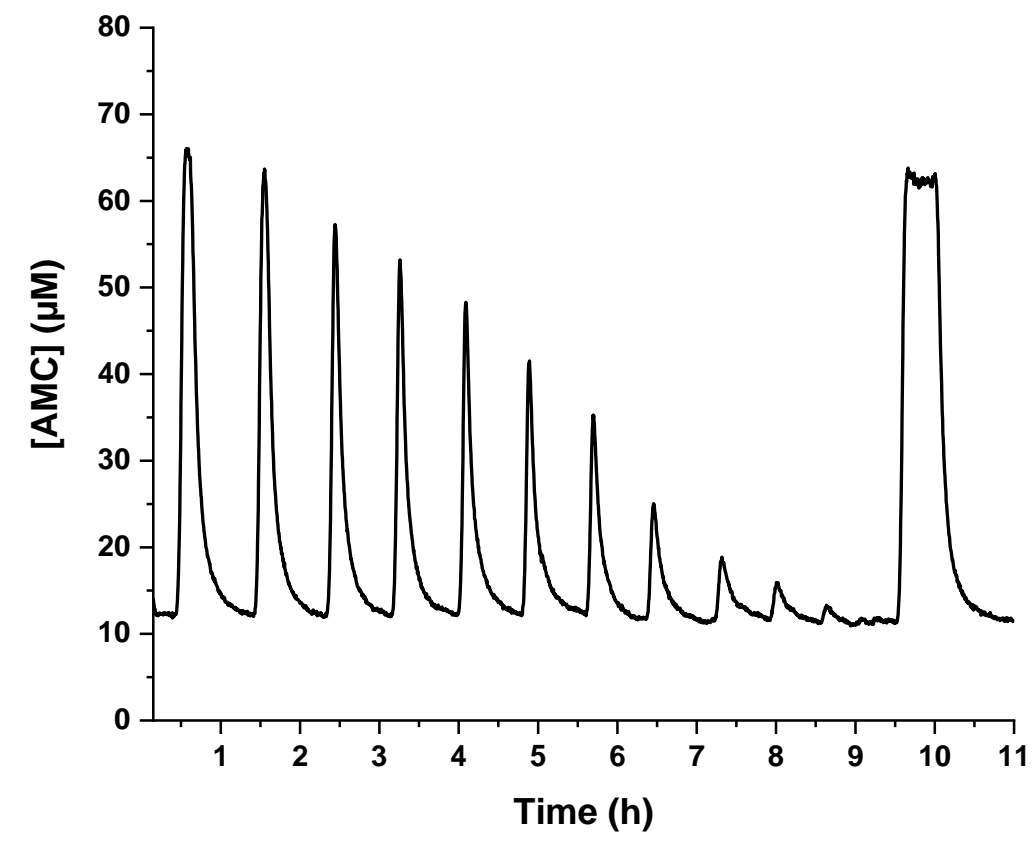

Figure S90. Results of the flow experiment: Temporal Filtering: Cr/PI system: Pulse Sequence no blue irradiation. 


\section{Experimental Details and Results: Pulse Sequence with blue irradiation}

The flow experiment was performed using a self-made CSTR with an inner volume of $196 \mu \mathrm{L}$ (stirring bar taken into account). Throughout the whole experiment, the overall flow rate was kept at $1200 \mu \mathrm{L} / \mathrm{h}$, resulting in a $k_{\text {flow }}$ of $6.1 \mathrm{~h}^{-1}$. The experimental conditions (concentration of compound in feed syringe, flowrate and final concentration of the respective compound in flow cuvette) for the flow experiment are outlined in Table S11.

Table S11. Experimental conditions for the flow experiment: concentration of compound in feed syringe, flowrate and final concentration of the respective compound in flow cuvette.

\begin{tabular}{cccc}
\hline Compound & $\begin{array}{c}\text { Flowrate } / \\
(\boldsymbol{\mu L} / \mathbf{h})\end{array}$ & $\begin{array}{c}\text { Concentration in Feed Syringe } \\
/ \boldsymbol{\mu M}\end{array}$ & $\begin{array}{c}\text { Concentration in } \\
\text { Flow Cuvette } / \boldsymbol{\mu M}\end{array}$ \\
\hline Cr & 400 & $0.12 \mu \mathrm{M}$ & $0.04 \mu \mathrm{M}$ \\
PI (20) & 400 & $600 \mu \mathrm{M}$ & $200 \mu \mathrm{M}$ \\
Suc-AAPF-AMC & 200 & $480 \mu \mathrm{M}$ & $80 \mu \mathrm{M}$ \\
Buffer Syringe 1 & 100 & $200 \mathrm{mM} \mathrm{Tris,} 20 \mathrm{mM} \mathrm{Ca}^{2+}$ and \\
Buffer Syringe 2 & \multirow{4}{*}{$0.1 \mathrm{wt} \% \mathrm{BSA}, \mathrm{pH}=7.8$} \\
\end{tabular}

The light pulse sequence (light source, intensity, timing and duration) applied throughout the experiment is outlined in Table S12. For $365 \mathrm{~nm}$ irradiation, a Thorlabs bandpass filter was placed in between the CSTR and the LED. The power of light $\left(100 \%(365 \mathrm{~nm})=37.1 \mathrm{~mW} / \mathrm{cm}^{2}, 100 \%(460 \mathrm{~nm})=\right.$ $143.3 \mathrm{~mW} / \mathrm{cm}^{2}$ ) was measured using a Thorlabs PM100A power meter equipped with a photodiode sensor. 
Table S12. Light pulse sequence applied throughout the performance of the flow experiment.

\begin{tabular}{|c|c|c|c|}
\hline Time & Light Source & Duration & Intensity \\
\hline Start & I & l & l \\
\hline $1.16 \mathrm{~h}$ & $\begin{array}{c}365 \mathrm{~nm} \& 460 \mathrm{~nm} \\
\text { Avonec }\end{array}$ & $\begin{array}{l}5 \text { min UV at } 100 \% \text { intensity, } 15 \mathrm{sec} \text { blue at } \\
100 \% \text { intensity, then light off }\end{array}$ & $100 \%$ for both lights \\
\hline $1.5 \mathrm{~h}$ & $\begin{array}{c}365 \mathrm{~nm} \& 460 \mathrm{~nm} \\
\text { Avonec }\end{array}$ & $\begin{array}{l}10 \mathrm{~min} \mathrm{UV} \text { at } 100 \% \text { intensity, } 15 \mathrm{sec} \text { blue } \\
\text { at } 100 \% \text { intensity, then light off }\end{array}$ & $100 \%$ for both lights \\
\hline $1.85 \mathrm{~h}$ & $\begin{array}{l}365 \mathrm{~nm} \& 460 \mathrm{~nm} \\
\text { Avonec }\end{array}$ & $\begin{array}{l}15 \text { min UV at } 100 \% \text { intensity, } 15 \mathrm{sec} \text { blue } \\
\text { at } 100 \% \text { intensity, then light off }\end{array}$ & $100 \%$ for both lights \\
\hline $2.3 \mathrm{~h}$ & $\begin{array}{l}365 \mathrm{~nm} \& 460 \mathrm{~nm} \\
\text { Avonec }\end{array}$ & $\begin{array}{l}30 \mathrm{~min} \text { UV at } 100 \% \text { intensity, } 15 \mathrm{sec} \text { blue } \\
\text { at } 100 \% \text { intensity, then light off }\end{array}$ & $100 \%$ for both lights \\
\hline $3.0 \mathrm{~h}$ & $\begin{array}{c}365 \mathrm{~nm} \& 460 \mathrm{~nm} \\
\text { Avonec }\end{array}$ & $\begin{array}{l}7.5 \mathrm{~min} \text { UV at } 100 \% \text { intensity, } 15 \mathrm{sec} \text { blue } \\
\text { at } 100 \% \text { intensity, then light off }\end{array}$ & $100 \%$ for both lights \\
\hline $3.4 \mathrm{~h}$ & $\begin{array}{c}365 \mathrm{~nm} \& 460 \mathrm{~nm} \\
\text { Avonec }\end{array}$ & $\begin{array}{l}6.5 \mathrm{~min} \text { UV at } 100 \% \text { intensity, } 15 \mathrm{sec} \text { blue } \\
\text { at } 100 \% \text { intensity, then light off }\end{array}$ & $100 \%$ for both lights \\
\hline $3.7 \mathrm{~h}$ & $\begin{array}{c}365 \mathrm{~nm} \& 460 \mathrm{~nm} \\
\text { Avonec }\end{array}$ & $\begin{array}{l}5.75 \mathrm{~min} \mathrm{UV} \text { at } 100 \% \text { intensity, } 15 \mathrm{sec} \\
\text { blue at } 100 \% \text { intensity, then light off }\end{array}$ & $100 \%$ for both lights \\
\hline $3.95 \mathrm{~h}$ & $\begin{array}{c}365 \mathrm{~nm} \& 460 \mathrm{~nm} \\
\text { Avonec }\end{array}$ & $\begin{array}{c}5 \text { min UV at } 100 \% \text { intensity, } 15 \mathrm{sec} \text { blue at } \\
100 \% \text { intensity, then light off }\end{array}$ & $100 \%$ for both lights \\
\hline $4.22 \mathrm{~h}$ & $\begin{array}{c}365 \mathrm{~nm} \& 460 \mathrm{~nm} \\
\text { Avonec }\end{array}$ & $\begin{array}{c}4 \text { min UV at } 100 \% \text { intensity, } 15 \mathrm{sec} \text { blue at } \\
100 \% \text { intensity, then light off }\end{array}$ & $100 \%$ for both lights \\
\hline $4.46 \mathrm{~h}$ & $\begin{array}{c}365 \mathrm{~nm} \& 460 \mathrm{~nm} \\
\text { Avonec }\end{array}$ & $\begin{array}{l}4 \mathrm{~min} \text { UV at } 100 \% \text { intensity, } 15 \mathrm{sec} \text { blue at } \\
100 \% \text { intensity, then light off }\end{array}$ & $100 \%$ for both lights \\
\hline $4.71 \mathrm{~h}$ & $\begin{array}{c}365 \mathrm{~nm} \& 460 \mathrm{~nm} \\
\text { Avonec }\end{array}$ & $\begin{array}{c}3 \text { min UV at } 100 \% \text { intensity, } 15 \mathrm{sec} \text { blue at } \\
100 \% \text { intensity, then light off }\end{array}$ & $100 \%$ for both lights \\
\hline $4.94 \mathrm{~h}$ & $\begin{array}{c}365 \mathrm{~nm} \& 460 \mathrm{~nm} \\
\text { Avonec }\end{array}$ & $\begin{array}{c}2 \text { min UV at } 100 \% \text { intensity, } 15 \mathrm{sec} \text { blue at } \\
100 \% \text { intensity, then light off }\end{array}$ & $100 \%$ for both lights \\
\hline $5.17 \mathrm{~h}$ & $\begin{array}{c}365 \mathrm{~nm} \& 460 \mathrm{~nm} \\
\text { Avonec }\end{array}$ & $\begin{array}{l}1 \mathrm{~min} \text { UV at } 100 \% \text { intensity, } 15 \mathrm{sec} \text { blue at } \\
100 \% \text { intensity, then light off }\end{array}$ & $100 \%$ for both lights \\
\hline $5.55 \mathrm{~h}$ & $\begin{array}{c}365 \mathrm{~nm} \& 460 \mathrm{~nm} \\
\text { Avonec }\end{array}$ & $\begin{array}{l}30 \mathrm{sec} U \mathrm{UV} \text { at } 100 \% \text { intensity, } 15 \mathrm{sec} \text { blue } \\
\text { at } 100 \% \text { intensity, then light off }\end{array}$ & $100 \%$ for both lights \\
\hline
\end{tabular}

The time/AMC concentration plot of this experiment, performed under out-of-equilibrium conditions using the previously described flow setup, is outlined in Figure S91. 


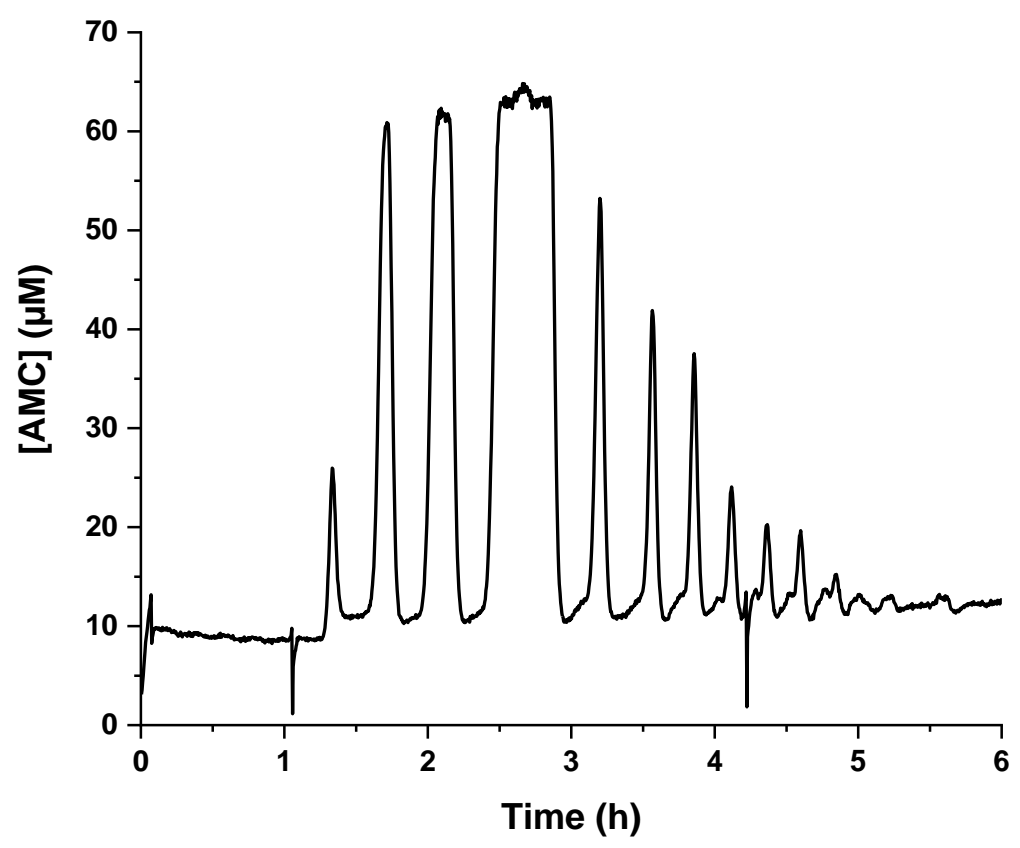

Figure S91. Results of the flow experiment: Temporal Filtering: Cr/PI system: Pulse Sequence with blue irradiation.

Plotting of the results: Pulse duration vs. [AMC] for both pulse sequences

To compare the experimental results of the two different applied pulse sequences, the UV pulse duration was plotted logarithmically against the maximum AMC concentration observed after the respective light pulse (Fig. S92).

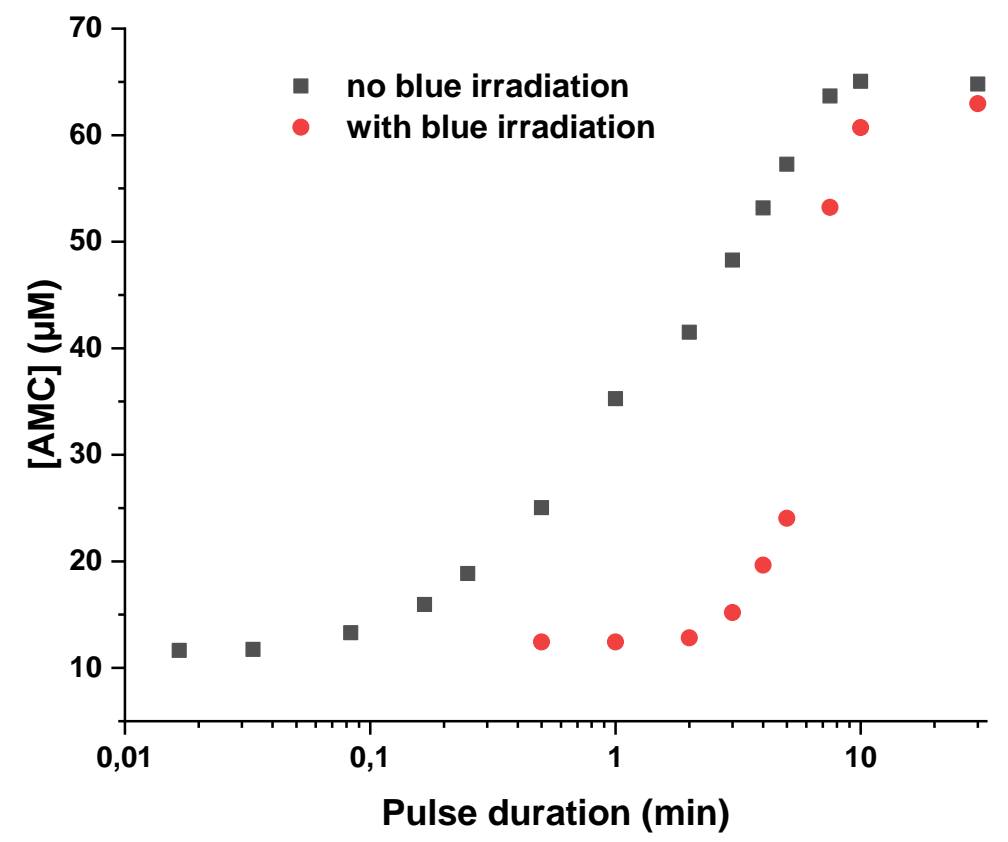

Figure S92. Plotting of the results: Pulse duration vs. [AMC] for both pulse sequences. 


\subsubsection{Alteration of Pulse Duration Cr/Ap System (Main Text Fig. 4C)}

\section{Experimental Details and Results: Pulse Sequence no blue irradiation}

The flow experiment was performed using a self-made CSTR with an inner volume of $196 \mu \mathrm{L}$ (stirring bar taken into account). Throughout the whole experiment, the overall flow rate was kept at $1300 \mu \mathrm{L} / \mathrm{h}$, resulting in a $k_{\text {flow }}$ of $6.1 \mathrm{~h}^{-1}$. The experimental conditions (concentration of compound in feed syringe, flowrate and final concentration of the respective compound in flow cuvette) for the flow experiment are outlined in Table S13.

Table S13. Experimental conditions for the flow experiment: concentration of compound in feed syringe, flowrate and final concentration of the respective compound in flow cuvette.

\begin{tabular}{cccc}
\hline Compound & $\begin{array}{c}\text { Flowrate / } \\
(\boldsymbol{\mu} \mathbf{L} / \mathbf{h})\end{array}$ & $\begin{array}{c}\text { Concentration in Feed Syringe } \\
/ \boldsymbol{\mu M}\end{array}$ & $\begin{array}{c}\text { Concentration in } \\
\text { Flow Cuvette } / \boldsymbol{\mu M}\end{array}$ \\
\hline Cr & 200 & $0.12 \mu \mathrm{M}$ & $0.02 \mu \mathrm{M}$ \\
PI (20) & 400 & $600 \mu \mathrm{M}$ & $200 \mu \mathrm{M}$ \\
Z-Phe-Arg-AMC & 400 & $300 \mu \mathrm{M}$ & $100 \mu \mathrm{M}$ \\
Buffer & 100 & $200 \mathrm{mM} \mathrm{Tris,} 20 \mathrm{mM} \mathrm{Ca}{ }^{2+}$ and & \\
Ap & 200 & $0.1 \mathrm{wt} \% \mathrm{BSA}, \mathrm{pH}=7.8$ & $0.1 \mathrm{U} / \mathrm{mL}$ \\
\hline
\end{tabular}

The light pulse sequence (light source, intensity, timing and duration) applied throughout the experiment is outlined in Table S14. For $365 \mathrm{~nm}$ irradiation, a Thorlabs bandpass filter was placed in between the CSTR and the LED. The power of light $\left(100 \%(365 \mathrm{~nm})=35.9 \mathrm{~mW} / \mathrm{cm}^{2}\right)$ was measured using a Thorlabs PM100A power meter equipped with a photodiode sensor. 
Table S14. Light pulse sequence applied throughout the performance of the flow experiment.

\begin{tabular}{|c|c|c|c|}
\hline Time & Light Source & Duration & Intensity \\
\hline Start & l & l & l \\
\hline $0.7 \mathrm{~h}$ & $365 \mathrm{~nm}$ Avonec & $60 \mathrm{~min}$ at $100 \%$ intensity, then off & $100 \%$ \\
\hline $2.89 \mathrm{~h}$ & $365 \mathrm{~nm}$ Avonec & 30 min at $100 \%$ intensity, then off & $100 \%$ \\
\hline $4.5 \mathrm{~h}$ & $365 \mathrm{~nm}$ Avonec & $22.5 \mathrm{~min}$ at $100 \%$ intensity, then off & $100 \%$ \\
\hline $5.98 \mathrm{~h}$ & $365 \mathrm{~nm}$ Avonec & $15 \mathrm{~min}$ at $100 \%$ intensity, then off & $100 \%$ \\
\hline $7.34 \mathrm{~h}$ & $365 \mathrm{~nm}$ Avonec & 10 min at $100 \%$ intensity, then off & $100 \%$ \\
\hline $8.58 \mathrm{~h}$ & $365 \mathrm{~nm}$ Avonec & $7.5 \mathrm{~min}$ at $100 \%$ intensity, then off & $100 \%$ \\
\hline $9.77 \mathrm{~h}$ & $365 \mathrm{~nm}$ Avonec & $5 \mathrm{~min}$ at $100 \%$ intensity, then off & $100 \%$ \\
\hline $10.98 \mathrm{~h}$ & $365 \mathrm{~nm}$ Avonec & $3 \mathrm{~min}$ at $100 \%$ intensity, then off & $100 \%$ \\
\hline $12.11 \mathrm{~h}$ & $365 \mathrm{~nm}$ Avonec & 2 min at $100 \%$ intensity, then off & $100 \%$ \\
\hline $13.25 \mathrm{~h}$ & $365 \mathrm{~nm}$ Avonec & $1 \mathrm{~min}$ at $100 \%$ intensity, then off & $100 \%$ \\
\hline $14.34 \mathrm{~h}$ & $365 \mathrm{~nm}$ Avonec & $30 \mathrm{sec}$ at $100 \%$ intensity, then off & $100 \%$ \\
\hline $15.45 \mathrm{~h}$ & $365 \mathrm{~nm}$ Avonec & $15 \mathrm{sec}$ at $100 \%$ intensity, then off & $100 \%$ \\
\hline $16.56 \mathrm{~h}$ & $365 \mathrm{~nm}$ Avonec & $8 \mathrm{sec}$ at $100 \%$ intensity, then off & $100 \%$ \\
\hline $17.67 \mathrm{~h}$ & $365 \mathrm{~nm}$ Avonec & $5 \mathrm{sec}$ at $100 \%$ intensity, then off & $100 \%$ \\
\hline $18.78 \mathrm{~h}$ & $365 \mathrm{~nm}$ Avonec & $2 \mathrm{sec}$ at $100 \%$ intensity, then off & $100 \%$ \\
\hline
\end{tabular}

The time/AMC concentration plot of this experiment, performed under out-of-equilibrium conditions using the previously described flow setup, is outlined in Figure S93.

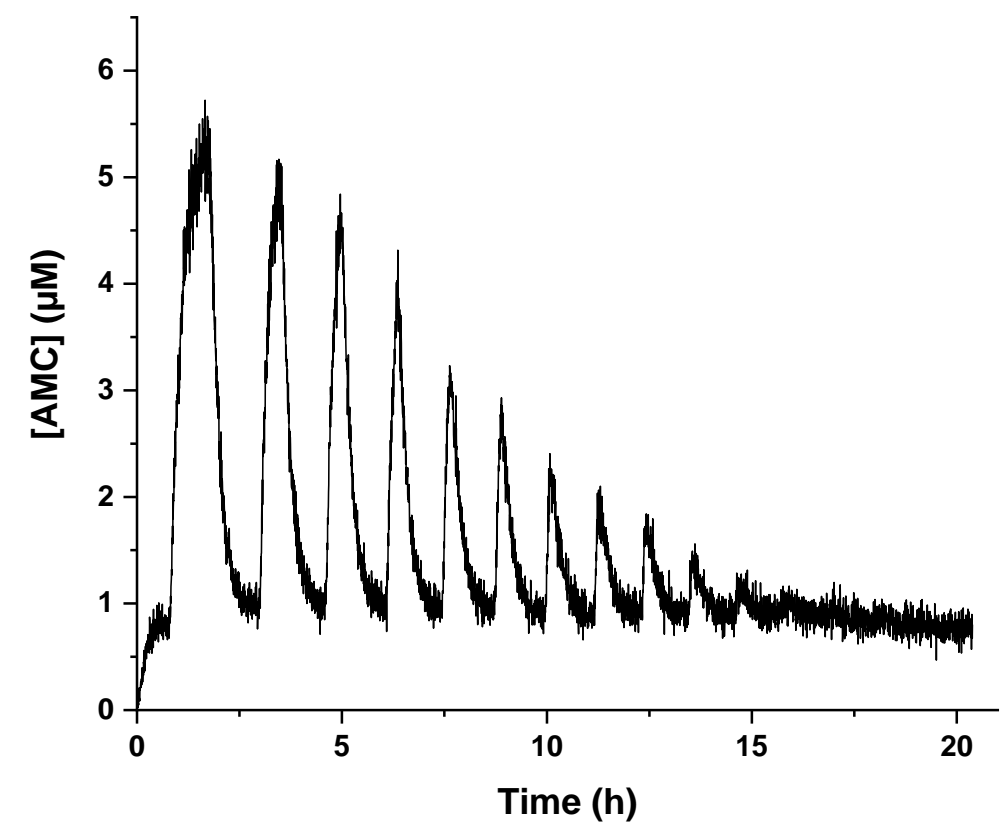

Figure S93. Results of the flow experiment: Temporal Filtering: Ap/Cr-based enzymatic cascade: Pulse Sequence no blue irradiation. 


\section{Experimental Details and Results: Pulse Sequence with blue irradiation}

The flow experiment was performed using a self-made CSTR with an inner volume of $196 \mu \mathrm{L}$ (stirring bar taken into account). Throughout the whole experiment, the overall flow rate was kept at $1300 \mu \mathrm{L} / \mathrm{h}$, resulting in a $k_{\text {flow }}$ of $6.1 \mathrm{~h}^{-1}$. The experimental conditions (concentration of compound in feed syringe, flowrate and final concentration of the respective compound in flow cuvette) for the flow experiment are outlined in Table S15.

Table S15. Experimental conditions for the flow experiment: concentration of compound in feed syringe, flowrate and final concentration of the respective compound in flow cuvette.

\begin{tabular}{cccc}
\hline Compound & $\begin{array}{c}\text { Flowrate } / \\
(\boldsymbol{\mu L} / \mathbf{h})\end{array}$ & $\begin{array}{c}\text { Concentration in Feed Syringe } \\
/ \boldsymbol{\mu M}\end{array}$ & $\begin{array}{c}\text { Concentration in } \\
\text { Flow Cuvette } / \boldsymbol{\mu M}\end{array}$ \\
\hline $\mathrm{Cr}$ & 200 & $0.12 \mu \mathrm{M}$ & $0.02 \mu \mathrm{M}$ \\
PI (20) & 400 & $600 \mu \mathrm{M}$ & $200 \mu \mathrm{M}$ \\
Z-Phe-Arg-AMC & 400 & $300 \mu \mathrm{M}$ & $100 \mu \mathrm{M}$ \\
Buffer & 100 & $200 \mathrm{mM} \mathrm{Tris,} 20 \mathrm{mM} \mathrm{Ca} \mathrm{Ca}^{2+}$ and & \\
Ap & 200 & $0.1 \mathrm{wt} \% \mathrm{BSA}, \mathrm{pH}=7.8$ & $0.1 \mathrm{U} / \mathrm{mL}$ \\
\hline
\end{tabular}

The light pulse sequence (light source, intensity, timing and duration) applied throughout the experiment is outlined in Table S16. For $365 \mathrm{~nm}$ irradiation, a Thorlabs bandpass filter was placed in between the CSTR and the LED. The power of light $\left(100 \%(365 \mathrm{~nm})=37.6 \mathrm{~mW} / \mathrm{cm}^{2}, 100 \%(460 \mathrm{~nm})=\right.$ $147.8 \mathrm{~mW} / \mathrm{cm}^{2}$ ) was measured using a Thorlabs PM100A power meter equipped with a photodiode sensor. 
Table S16. Light pulse sequence applied throughout the performance of the flow experiment.

\begin{tabular}{|c|c|c|c|}
\hline Time & Light Source & Duration & Intensity \\
\hline Start & l & l & l \\
\hline $0.7 \mathrm{~h}$ & $\begin{array}{c}365 \mathrm{~nm} \& 460 \mathrm{~nm} \\
\text { Avonec }\end{array}$ & $\begin{array}{l}60 \mathrm{~min} \text { UV at } 100 \% \text { intensity, } 10 \mathrm{sec} \text { blue } \\
\text { at } 100 \% \text { intensity, then light off }\end{array}$ & $100 \%$ for both lights \\
\hline $2.99 \mathrm{~h}$ & $\begin{array}{l}365 \mathrm{~nm} \& 460 \mathrm{~nm} \\
\text { Avonec }\end{array}$ & $\begin{array}{l}30 \mathrm{~min} \text { UV at } 100 \% \text { intensity, } 10 \mathrm{sec} \text { blue } \\
\text { at } 100 \% \text { intensity, then light off }\end{array}$ & $100 \%$ for both lights \\
\hline $4.6 \mathrm{~h}$ & $\begin{array}{l}365 \mathrm{~nm} \& 460 \mathrm{~nm} \\
\text { Avonec }\end{array}$ & $\begin{array}{l}22.5 \mathrm{~min} \text { UV at } 100 \% \text { intensity, } 10 \mathrm{sec} \\
\text { blue at } 100 \% \text { intensity, then light off }\end{array}$ & $100 \%$ for both lights \\
\hline $6.08 \mathrm{~h}$ & $\begin{array}{l}365 \mathrm{~nm} \& 460 \mathrm{~nm} \\
\text { Avonec }\end{array}$ & $\begin{array}{l}15 \text { min UV at } 100 \% \text { intensity, } 10 \mathrm{sec} \text { blue } \\
\text { at } 100 \% \text { intensity, then light off }\end{array}$ & $100 \%$ for both lights \\
\hline $7.54 \mathrm{~h}$ & $\begin{array}{l}365 \mathrm{~nm} \& 460 \mathrm{~nm} \\
\text { Avonec }\end{array}$ & $\begin{array}{l}10 \text { min UV at } 100 \% \text { intensity, } 10 \mathrm{sec} \text { blue } \\
\text { at } 100 \% \text { intensity, then light off }\end{array}$ & $100 \%$ for both lights \\
\hline $8.88 \mathrm{~h}$ & $\begin{array}{c}365 \mathrm{~nm} \& 460 \mathrm{~nm} \\
\text { Avonec }\end{array}$ & $\begin{array}{l}7.5 \text { min UV at } 100 \% \text { intensity, } 10 \mathrm{sec} \text { blue } \\
\text { at } 100 \% \text { intensity, then light off }\end{array}$ & $100 \%$ for both lights \\
\hline $9.97 \mathrm{~h}$ & $\begin{array}{c}365 \mathrm{~nm} \& 460 \mathrm{~nm} \\
\text { Avonec }\end{array}$ & $\begin{array}{c}5 \text { min UV at } 100 \% \text { intensity, } 10 \mathrm{sec} \text { blue at } \\
100 \% \text { intensity, then light off }\end{array}$ & $100 \%$ for both lights \\
\hline $11.18 \mathrm{~h}$ & $\begin{array}{c}365 \mathrm{~nm} \& 460 \mathrm{~nm} \\
\text { Avonec }\end{array}$ & $\begin{array}{c}3 \text { min UV at } 100 \% \text { intensity, } 10 \mathrm{sec} \text { blue at } \\
100 \% \text { intensity, then light off }\end{array}$ & $100 \%$ for both lights \\
\hline $12.31 \mathrm{~h}$ & $\begin{array}{c}365 \mathrm{~nm} \& 460 \mathrm{~nm} \\
\text { Avonec }\end{array}$ & $\begin{array}{c}2 \text { min UV at } 100 \% \text { intensity, } 10 \mathrm{sec} \text { blue at } \\
100 \% \text { intensity, then light off }\end{array}$ & $100 \%$ for both lights \\
\hline $13.45 \mathrm{~h}$ & $\begin{array}{c}365 \mathrm{~nm} \& 460 \mathrm{~nm} \\
\text { Avonec }\end{array}$ & $\begin{array}{l}1 \text { min UV at } 100 \% \text { intensity, } 10 \mathrm{sec} \text { blue at } \\
100 \% \text { intensity, then light off }\end{array}$ & $100 \%$ for both lights \\
\hline $14.54 \mathrm{~h}$ & $\begin{array}{c}365 \mathrm{~nm} \& 460 \mathrm{~nm} \\
\text { Avonec }\end{array}$ & $\begin{array}{l}30 \mathrm{sec} \mathrm{UV} \text { at } 100 \% \text { intensity, } 10 \mathrm{sec} \text { blue } \\
\text { at } 100 \% \text { intensity, then light off }\end{array}$ & $100 \%$ for both lights \\
\hline $15.75 \mathrm{~h}$ & $\begin{array}{c}365 \mathrm{~nm} \& 460 \mathrm{~nm} \\
\text { Avonec }\end{array}$ & $\begin{array}{l}15 \mathrm{sec} \text { UV at } 100 \% \text { intensity, } 10 \mathrm{sec} \text { blue } \\
\text { at } 100 \% \text { intensity, then light off }\end{array}$ & $100 \%$ for both lights \\
\hline $16.86 \mathrm{~h}$ & $\begin{array}{c}365 \mathrm{~nm} \& 460 \mathrm{~nm} \\
\text { Avonec }\end{array}$ & $\begin{array}{c}8 \mathrm{sec} \text { UV at } 100 \% \text { intensity, } 10 \mathrm{sec} \text { blue at } \\
100 \% \text { intensity, then light off }\end{array}$ & $100 \%$ for both lights \\
\hline $17.97 \mathrm{~h}$ & $\begin{array}{c}365 \mathrm{~nm} \& 460 \mathrm{~nm} \\
\text { Avonec }\end{array}$ & $\begin{array}{c}5 \mathrm{sec} \text { UV at } 100 \% \text { intensity, } 10 \mathrm{sec} \text { blue at } \\
100 \% \text { intensity, then light off }\end{array}$ & $100 \%$ for both lights \\
\hline $18.98 \mathrm{~h}$ & $\begin{array}{l}365 \mathrm{~nm} \& 460 \mathrm{~nm} \\
\text { Avonec }\end{array}$ & $\begin{array}{c}2 \mathrm{sec} \mathrm{UV} \text { at } 100 \% \text { intensity, } 10 \mathrm{sec} \text { blue at } \\
100 \% \text { intensity, then light off }\end{array}$ & $100 \%$ for both lights \\
\hline
\end{tabular}

The time/AMC concentration plot of this experiment, performed under out-of-equilibrium conditions using the previously described flow setup, is outlined in Figure S94. 


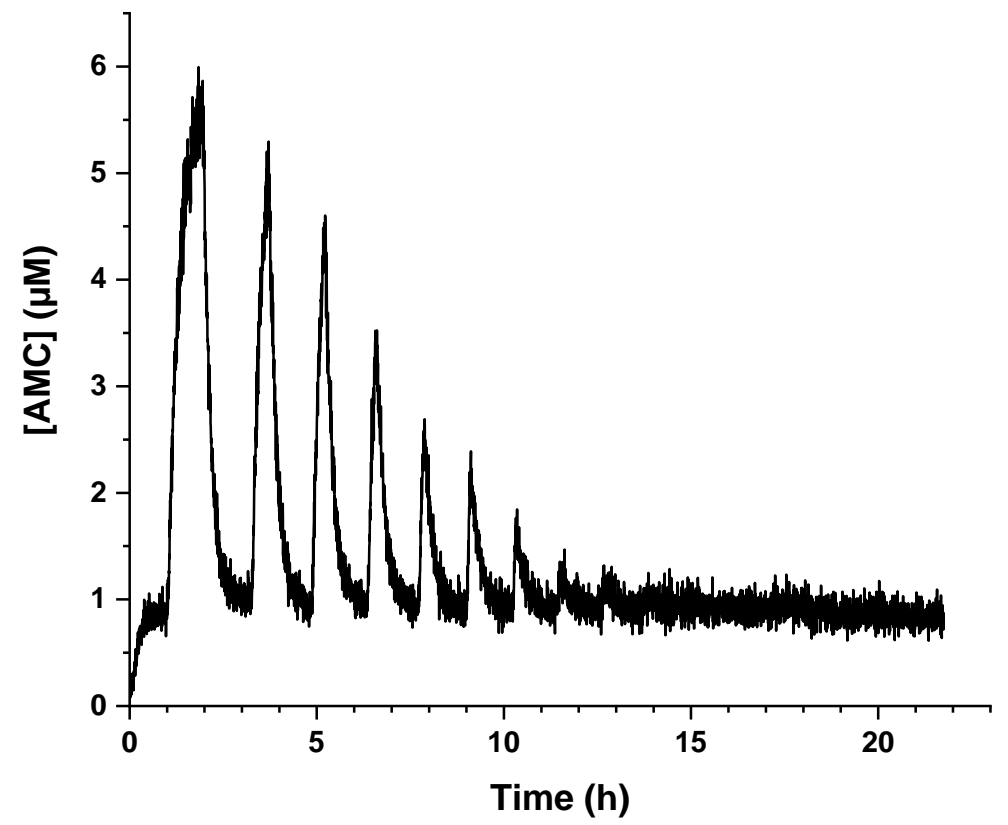

Figure S94. Results of the flow experiment: Temporal Filtering: $\mathrm{Cr} / \mathrm{PI}$ system: Pulse Sequence with blue irradiation.

\section{Plotting of the results: Pulse duration vs. [AMC] for both pulse sequences}

To compare the experimental results of the two different applied pulse sequences, the UV pulse duration was plotted logarithmically against the normalized AMC output $\left(\left([\mathrm{AMC}]_{\mathrm{t}}-[\mathrm{AMC}]_{460 \mathrm{~nm}}\right) /[\mathrm{AMC}]_{365 \mathrm{~nm}}\right)$ observed after the respective light pulse (Fig. S95).

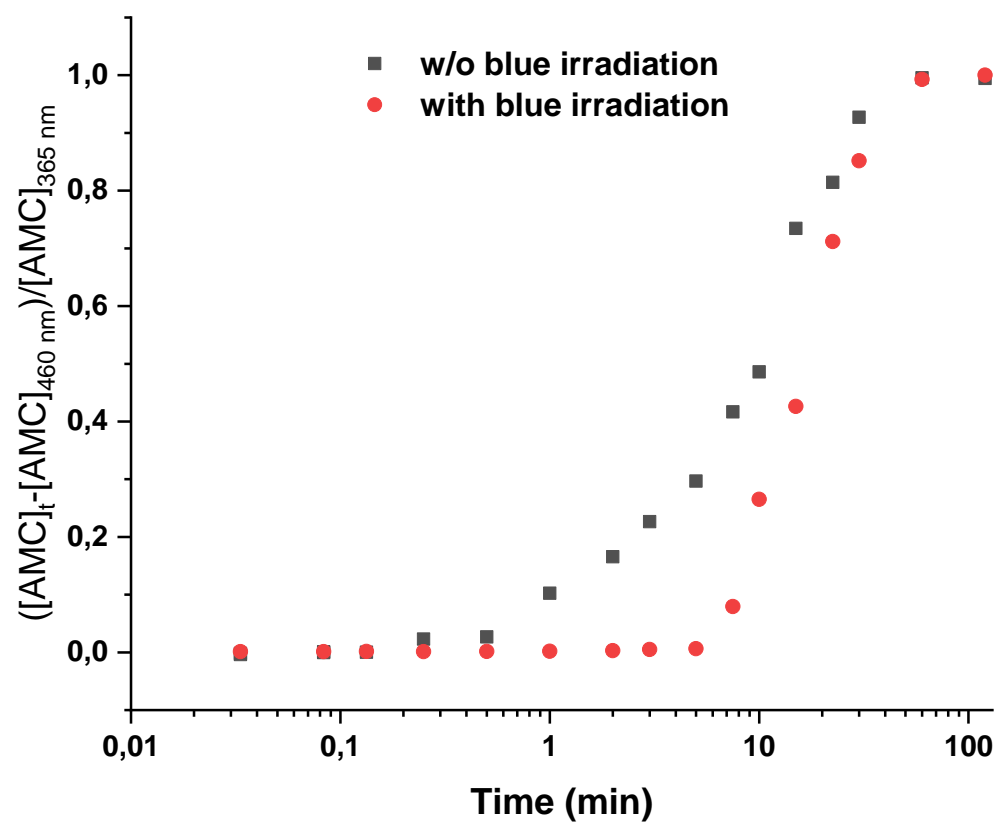

Figure S95. Plotting of the results: Pulse duration vs. [AMC] for both pulse sequences. 


\section{Repetition of experiments presented in Main Text Fig. 4C}

To emphasize the reproducibility and accuracy of our experimental setup and results, we performed the experiments described above one more time. As depicted in Figure S96, similar Hill coefficients could be obtained, thereby pointing towards high reproducibility and robustness of our experimental setup and analysis. As we are using frozen enzyme stock solutions and substrates, we believe that the error in weighing out the photoswitchable inhibitor is the most sensitive parameter with respect to the experimental conditions. The fluorescence setup itself is highly reproducible, as all subunits are being precisely controlled by software (e.g. the timing, duration and intensity of irradiation) and the difference in light intensity is being accounted for by normalization with respect to the highest observed peak.

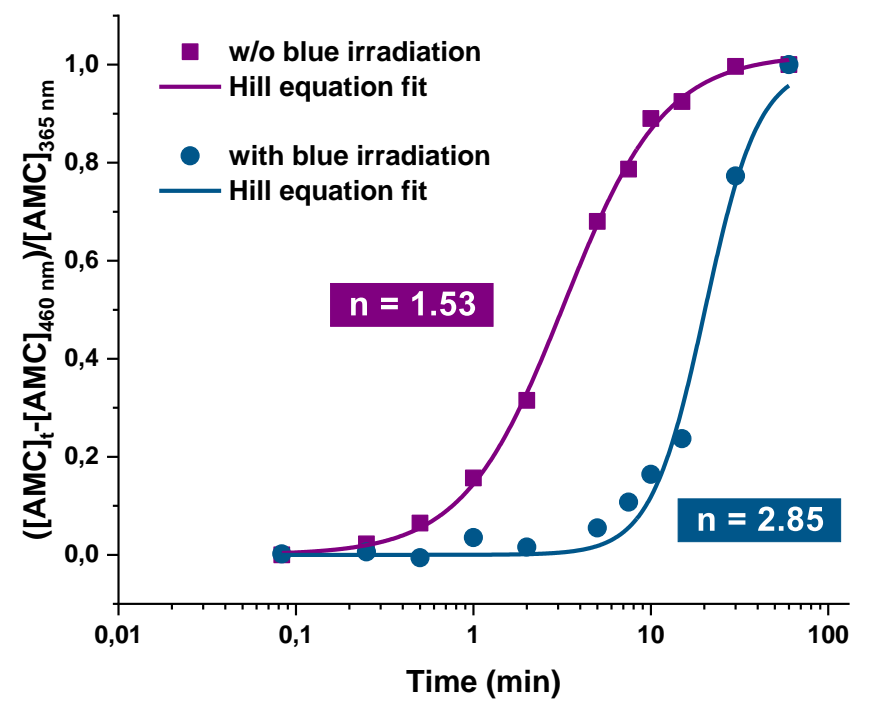

Figure S96. Plotting of the results: UV pulse duration vs. [AMC] for both pulse sequences and plotting to determine the hill coefficients for both pulse sequences. 


\subsubsection{Alteration of Pulse Frequency (Main Text Fig. 5)}

\section{Experimental Details and Results: Pulse Sequence no blue irradiation}

The flow experiment was performed using a self-made CSTR with an inner volume of $196 \mu \mathrm{L}$ (stirring bar taken into account). Throughout the whole experiment, the overall flow rate was kept at $1200 \mu \mathrm{L} / \mathrm{h}$, resulting in a $k_{\text {flow }}$ of $6.1 \mathrm{~h}^{-1}$. The experimental conditions (concentration of compound in feed syringe, flowrate and final concentration of the respective compound in flow cuvette) for the flow experiment are outlined in Table S17.

Table S17. Experimental conditions for the flow experiment: concentration of compound in feed syringe, flowrate and final concentration of the respective compound in flow cuvette.

\begin{tabular}{|c|c|c|c|}
\hline Compound & $\begin{array}{c}\text { Flowrate / } \\
(\mu \mathrm{L} / \mathrm{h})\end{array}$ & $\begin{array}{c}\text { Concentration in Feed Syringe } \\
\qquad / \mu \mathrm{M}\end{array}$ & $\begin{array}{c}\text { Concentration in } \\
\text { Flow Cuvette / } \mu \mathrm{M}\end{array}$ \\
\hline $\mathrm{Cr}$ & 400 & $0.12 \mu \mathrm{M}$ & $0.04 \mu \mathrm{M}$ \\
\hline PI (20) & 400 & $600 \mu \mathrm{M}$ & $200 \mu \mathrm{M}$ \\
\hline Suc-AAPF-AMC & 200 & $480 \mu \mathrm{M}$ & $80 \mu \mathrm{M}$ \\
\hline Buffer Syringe 1 & 100 & $\begin{array}{c}200 \mathrm{mM} \text { Tris, } 20 \mathrm{mM} \mathrm{Ca}^{2+} \text { and } \\
0.1 \mathrm{wt} \% \text { BSA, } \mathrm{pH}=7.8\end{array}$ & \\
\hline Buffer Syringe 2 & 100 & $\begin{array}{c}200 \mathrm{mM} \text { Tris, } 20 \mathrm{mM} \mathrm{Ca}^{2+} \text { and } \\
0.1 \mathrm{wt} \% \text { BSA, } \mathrm{pH}=7.8\end{array}$ & \\
\hline
\end{tabular}

The light pulse sequence (light source, intensity, timing and duration) applied throughout the experiment is outlined in Table S18. For $365 \mathrm{~nm}$ irradiation, a Thorlabs bandpass filter was placed in between the CSTR and the LED. The power of light $\left(100 \%(365 \mathrm{~nm})=35.1 \mathrm{~mW} / \mathrm{cm}^{2}\right)$ was measured using a Thorlabs PM100A power meter equipped with a photodiode sensor.

Table S18. Light pulse sequence applied throughout the performance of the flow experiment.

\begin{tabular}{cccc}
\hline Time & Light Source & Duration & Offset between 4 UV pulses \\
\hline Start & $/$ & $/$ & $/$ \\
$0.51 \mathrm{~h}^{\mathrm{a}}$ & $365 \mathrm{~nm}$ Avonec & $30 \mathrm{sec}$ at 100\% intensity, then off (4x) & $10 \mathrm{sec}$ \\
$1.32 \mathrm{~h}$ & $365 \mathrm{~nm}$ Avonec & $30 \mathrm{sec}$ at 100\% intensity, then off (4x) & $30 \mathrm{~min}$ \\
$3.94 \mathrm{~h}$ & $365 \mathrm{~nm}$ Avonec & $30 \mathrm{sec}$ at 100\% intensity, then off (4x) & $15 \mathrm{~min}$ \\
$5.86 \mathrm{~h}$ & $365 \mathrm{~nm}$ Avonec & $30 \mathrm{sec}$ at 100\% intensity, then off $(4 \mathrm{x})$ & $10 \mathrm{~min}$ \\
$7.44 \mathrm{~h}$ & $365 \mathrm{~nm}$ Avonec & $30 \mathrm{sec}$ at 100\% intensity, then off $(4 \mathrm{x})$ & $5 \mathrm{~min}$ \\
$8.70 \mathrm{~h}$ & $365 \mathrm{~nm}$ Avonec & $30 \mathrm{sec}$ at 100\% intensity, then off $(4 \mathrm{x})$ & $2 \mathrm{~min}$ \\
$9.74 \mathrm{~h}$ & $365 \mathrm{~nm}$ Avonec & $30 \mathrm{sec}$ at 100\% intensity, then off $(4 \mathrm{x})$ & $1 \mathrm{~min}$ \\
$10.43 \mathrm{~h}$ & $365 \mathrm{~nm}$ Avonec & $30 \mathrm{sec}$ at 100\% intensity, then off $(4 \mathrm{x})$ & $1 \mathrm{sec}$ \\
$11.02 \mathrm{~h}$ & $365 \mathrm{~nm}$ Avonec & $30 \mathrm{sec}$ at 100\% intensity, then off $(4 \mathrm{x})$ & $60 \mathrm{~min}$ \\
\hline
\end{tabular}

a) A short blue light pulse $(10 \mathrm{sec})$ was applied after the maximum AMC concentration was reached to quickly reset the system. 
The time/AMC concentration plot of this experiment, performed under out-of-equilibrium conditions using the previously described flow setup, is outlined in Figure S97.

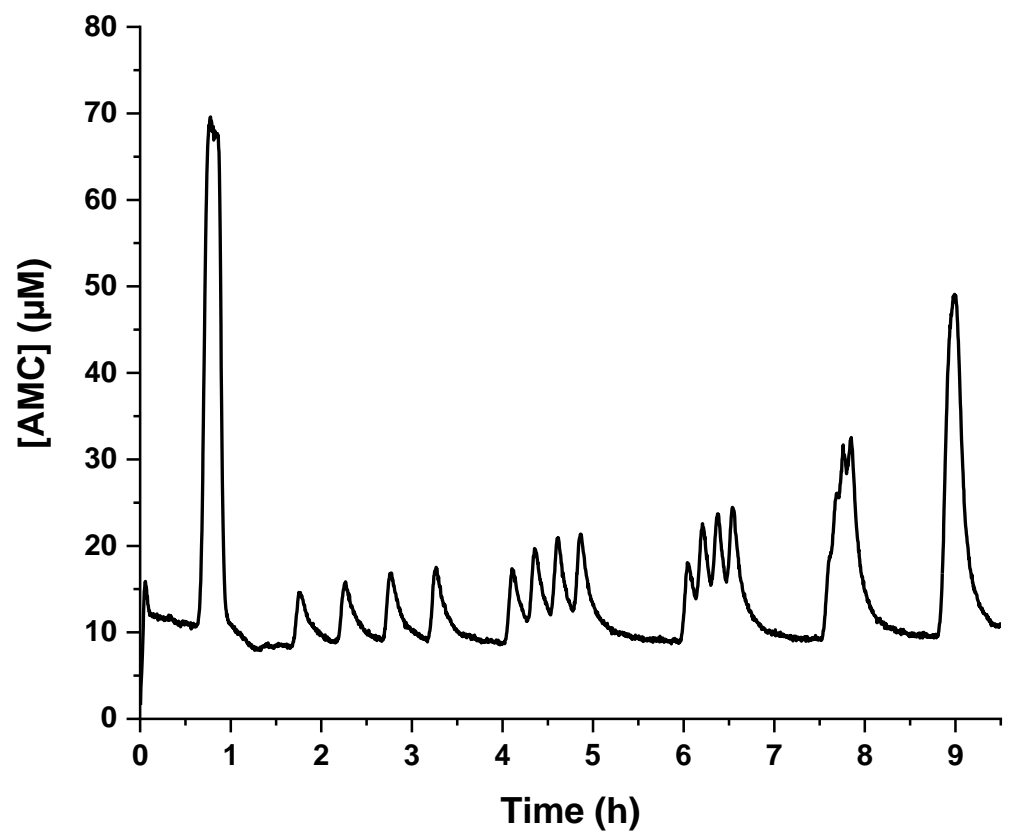

Figure S97. Results of the flow experiment: Temporal Filtering: Cr/PI system: Pulse Sequence no blue irradiation.

\section{Experimental Details and Results: Pulse Sequence with blue irradiation}

The flow experiment was performed using a self-made CSTR with an inner volume of $196 \mu \mathrm{L}$ (stirring bar taken into account). Throughout the whole experiment, the overall flow rate was kept at $1200 \mu \mathrm{L} / \mathrm{h}$, resulting in a $k_{\text {flow }}$ of $6.1 \mathrm{~h}^{-1}$. The experimental conditions (concentration of compound in feed syringe, flowrate and final concentration of the respective compound in flow cuvette) for the flow experiment are outlined in Table S19.

Table S19. Experimental conditions for the flow experiment: concentration of compound in feed syringe, flowrate and final concentration of the respective compound in flow cuvette.

\begin{tabular}{|c|c|c|c|}
\hline Compound & $\begin{array}{l}\text { Flowrate / } \\
(\mu \mathrm{L} / \mathrm{h})\end{array}$ & $\begin{array}{l}\text { Concentration in Feed Syringe } \\
\qquad / \mu \mathrm{M}\end{array}$ & $\begin{array}{l}\text { Concentration in } \\
\text { Flow Cuvette / } \mu \mathrm{M}\end{array}$ \\
\hline $\mathrm{Cr}$ & 400 & $0.12 \mu \mathrm{M}$ & $0.04 \mu \mathrm{M}$ \\
\hline PI (20) & 400 & $600 \mu \mathrm{M}$ & $200 \mu \mathrm{M}$ \\
\hline Suc-AAPF-AMC & 200 & $480 \mu \mathrm{M}$ & $80 \mu \mathrm{M}$ \\
\hline Buffer Syringe 1 & 100 & $\begin{array}{c}200 \mathrm{mM} \text { Tris, } 20 \mathrm{mM} \mathrm{Ca}^{2+} \text { and } \\
0.1 \mathrm{wt} \% \text { BSA, } \mathrm{pH}=7.8\end{array}$ & \\
\hline Buffer Syringe 2 & 100 & $\begin{array}{c}200 \mathrm{mM} \text { Tris, } 20 \mathrm{mM} \mathrm{Ca}^{2+} \text { and } \\
0.1 \mathrm{wt} \% \text { BSA, } \mathrm{pH}=7.8\end{array}$ & \\
\hline
\end{tabular}

The light pulse sequence (light source, intensity, timing and duration) applied throughout the experiment is outlined in Table S20. For $365 \mathrm{~nm}$ irradiation, a Thorlabs bandpass filter was placed in between the CSTR and the LED. The power of light $\left(100 \%(365 \mathrm{~nm})=35.9 \mathrm{~mW} / \mathrm{cm}^{2}, 100 \%(460 \mathrm{~nm})=\right.$ 
$155.8 \mathrm{~mW} / \mathrm{cm}^{2}$ ) was measured using a Thorlabs PM100A power meter equipped with a photodiode sensor.

Table S20. Light pulse sequence applied throughout the performance of the flow experiment.

\begin{tabular}{|c|c|c|c|}
\hline Time & Light Source & Duration & $\begin{array}{c}\text { Offset between } 4 \mathrm{UV} \\
\text { pulses }\end{array}$ \\
\hline Start & / & I & I \\
\hline $0.25 \mathrm{~h}^{\mathrm{a}}$ & $\begin{array}{c}365 \mathrm{~nm} \& 460 \mathrm{~nm} \\
\text { Avonec }\end{array}$ & $\begin{array}{l}15 \text { min UV at } 100 \% \text {, then } 15 \mathrm{sec} \text { blue at } \\
100 \% \text { intensity, then light off }\end{array}$ & I \\
\hline $1.08 \mathrm{~h}$ & $\begin{array}{c}365 \mathrm{~nm} \& 460 \mathrm{~nm} \\
\text { Avonec }\end{array}$ & $\begin{array}{c}30 \mathrm{sec} \text { UV at } 100 \% \text {, then } 5 \mathrm{sec} \text { blue at } \\
100 \% \text { intensity, then light off }\end{array}$ & $30 \mathrm{~min}$ \\
\hline $3.11 \mathrm{~h}$ & $\begin{array}{c}365 \mathrm{~nm} \& 460 \mathrm{~nm} \\
\text { Avonec }\end{array}$ & $\begin{array}{l}30 \mathrm{sec} \mathrm{UV} \text { at } 100 \% \text {, then } 5 \mathrm{sec} \text { blue at } \\
100 \% \text { intensity, then light off }\end{array}$ & $15 \mathrm{~min}$ \\
\hline $4.15 \mathrm{~h}$ & $\begin{array}{c}365 \mathrm{~nm} \& 460 \mathrm{~nm} \\
\text { Avonec }\end{array}$ & $\begin{array}{c}30 \mathrm{sec} \text { UV at } 100 \% \text {, then } 5 \mathrm{sec} \text { blue at } \\
100 \% \text { intensity, then light off }\end{array}$ & $10 \mathrm{~min}$ \\
\hline $4.93 \mathrm{~h}$ & $\begin{array}{c}365 \mathrm{~nm} \& 460 \mathrm{~nm} \\
\text { Avonec }\end{array}$ & $\begin{array}{l}30 \mathrm{sec} \text { UV at } 100 \% \text {, then } 5 \mathrm{sec} \text { blue at } \\
100 \% \text { intensity, then light off }\end{array}$ & $5 \min$ \\
\hline $5.46 \mathrm{~h}$ & $\begin{array}{c}365 \mathrm{~nm} \& 460 \mathrm{~nm} \\
\text { Avonec }\end{array}$ & $\begin{array}{l}30 \mathrm{sec} \text { UV at } 100 \% \text {, then } 5 \mathrm{sec} \text { blue at } \\
100 \% \text { intensity, then light off }\end{array}$ & $2 \min$ \\
\hline $5.93 \mathrm{~h}$ & $\begin{array}{c}365 \mathrm{~nm} \& 460 \mathrm{~nm} \\
\text { Avonec }\end{array}$ & $\begin{array}{l}30 \mathrm{sec} \text { UV at } 100 \% \text {, then } 5 \mathrm{sec} \text { blue at } \\
100 \% \text { intensity, then light off }\end{array}$ & $1 \mathrm{~min}$ \\
\hline $6.36 \mathrm{~h}$ & $\begin{array}{c}365 \mathrm{~nm} \& 460 \mathrm{~nm} \\
\text { Avonec }\end{array}$ & $\begin{array}{c}30 \mathrm{sec} \text { UV at } 100 \% \text {, then } 5 \mathrm{sec} \text { blue at } \\
100 \% \text { intensity, then light off }\end{array}$ & $30 \mathrm{sec}$ \\
\hline $6.74 \mathrm{~h}$ & $\begin{array}{c}365 \mathrm{~nm} \& 460 \mathrm{~nm} \\
\text { Avonec }\end{array}$ & $\begin{array}{l}30 \mathrm{sec} \text { UV at } 100 \% \text {, then } 5 \mathrm{sec} \text { blue at } \\
100 \% \text { intensity, then light off }\end{array}$ & $15 \mathrm{sec}$ \\
\hline $7.1 \mathrm{~h}$ & $\begin{array}{c}365 \mathrm{~nm} \& 460 \mathrm{~nm} \\
\text { Avonec }\end{array}$ & $\begin{array}{c}30 \mathrm{sec} \text { UV at } 100 \% \text {, then } 5 \mathrm{sec} \text { blue at } \\
100 \% \text { intensity, then light off }\end{array}$ & $5 \mathrm{sec}$ \\
\hline $7.53 \mathrm{~h}$ & $\begin{array}{c}365 \mathrm{~nm} \& 460 \mathrm{~nm} \\
\text { Avonec }\end{array}$ & $\begin{array}{l}30 \mathrm{sec} \text { UV at } 100 \% \text {, then } 5 \mathrm{sec} \text { blue at } \\
100 \% \text { intensity, then light off }\end{array}$ & $3 \mathrm{sec}$ \\
\hline $7.83 \mathrm{~h}$ & $\begin{array}{c}365 \mathrm{~nm} \& 460 \mathrm{~nm} \\
\text { Avonec }\end{array}$ & $\begin{array}{c}30 \mathrm{sec} \text { UV at } 100 \% \text {, then } 5 \mathrm{sec} \text { blue at } \\
100 \% \text { intensity, then light off }\end{array}$ & $2 \mathrm{sec}$ \\
\hline $8.22 \mathrm{~h}$ & $\begin{array}{l}365 \mathrm{~nm} \& 460 \mathrm{~nm} \\
\text { Avonec }\end{array}$ & $\begin{array}{c}30 \mathrm{sec} \text { UV at } 100 \% \text {, then } 5 \mathrm{sec} \text { blue at } \\
100 \% \text { intensity, then light off }\end{array}$ & $1 \mathrm{sec}$ \\
\hline
\end{tabular}

a) Identification of maximum AMC value obtained at $365 \mathrm{~nm}$ irradiation.

The time/AMC concentration plot of this experiment, performed under out-of-equilibrium conditions using the previously described flow setup, is outlined in Figure S98. 


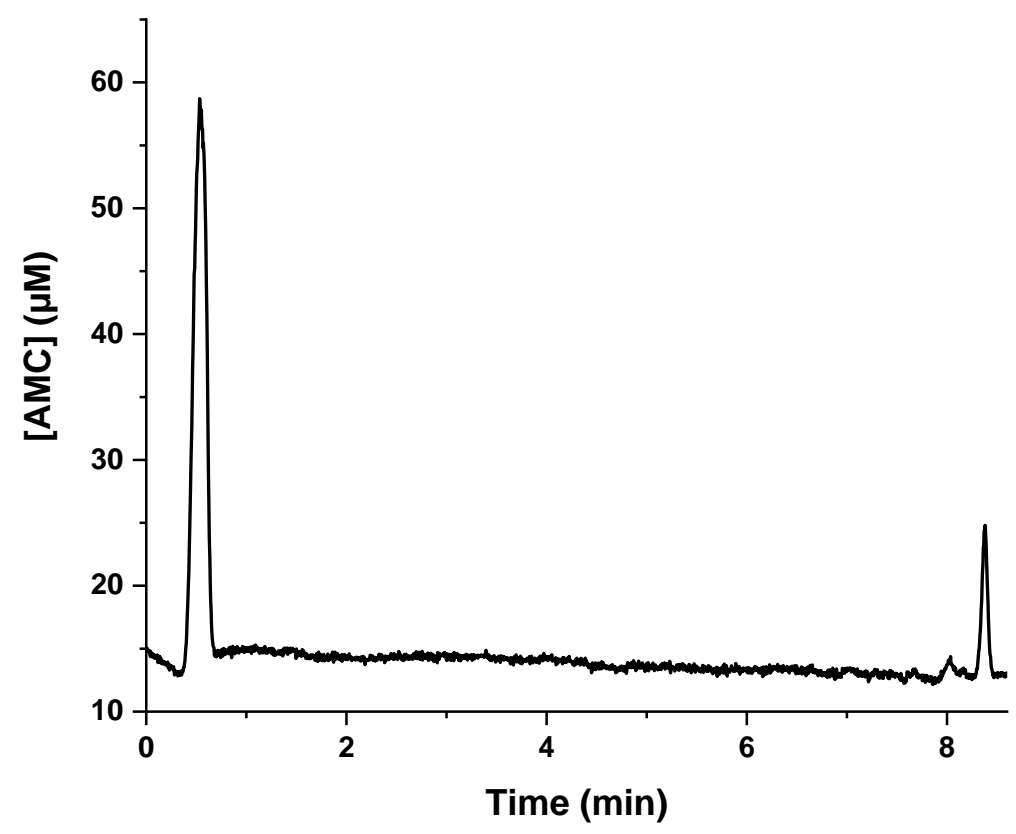

Figure S98. Results of the flow experiment: Temporal Filtering: Cr/PI system: Pulse Sequence with blue irradiation.

\section{Plotting of the results: Pulse duration vs. [AMC] for both pulse sequences}

To compare the experimental results of the two different applied pulse sequences, the offset of UV pulses was plotted logarithmically against the normalized maximum AMC output (([AMC $]_{t^{-}}$ $\left.[\mathrm{AMC}]_{460 \mathrm{~nm}}\right) /[\mathrm{AMC}]_{365 \mathrm{~nm}}$ ) observed after the respective set of light pulses with a certain offset in between them (Fig. S99).

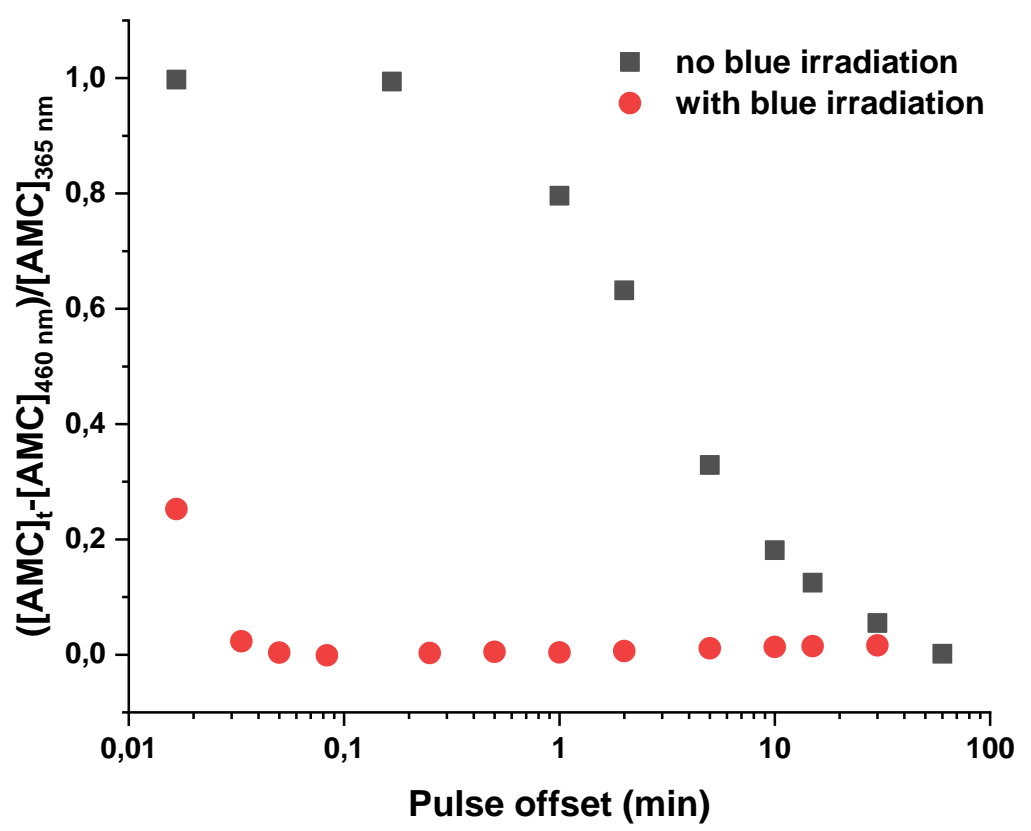

Figure S99. Plotting of the results: Pulse offset vs. normalized AMC output observed after a set of pulses with a certain offset. 


\section{Arduino \& Python Scripts for Controlling LEDs}

All scripts for controlling the LED setup have been uploaded to Github: https://github.com/A2P2/ultrasensitivity-by-light-pulses

\section{A brief summary of the scripts can be found below:}

For precisely regulating the intensity, timing, and duration of 365 and $460 \mathrm{~nm}$ irradiation, we wrote two scripts (Arduino and Python) to control the respective LEDs, which are both powered by connecting them to two Thorlabs LED1B drivers.

The trigger interface of the Thorlabs LED1B drivers are connected to two analog pins of an Arduino device. The intensity of these pins can be regulated from 0 (off) to 255 (max 100\% intensity), in steps of 1 unit. The Arduino script is uploaded on the Arduino board. The script is fed with values for irradiation source, intensity, timing and duration from a Python script operated on a PC.

The Python script provides a command line interface on a PC to program the sequence of irradiation. The values for irradiation source, intensity, timing and duration are provided by the user via an input function or a prepared csv table. 


\section{Model Simulations of the Ultrasensitive System (Main Text Fig. 4B)}

All scripts for modelling of the ultrasensitive system have been uploaded to Github: https://github.com/A2P2/ultrasensitivity-by-light-pulses

\section{A brief description can be found below:}

To model the ultrasensitive response of the system to light pulses, we simulated a system of chemical equations using Ordinary Differential Equations. The modeling was performed in Python. A detailed explanation on how to use the model and which parameters can be altered is given along the Python script, which can be accessed using the github link from above.

The chemical reactions describing the concentrations in the flow reactor (CSTR) are the following:

$$
\begin{gathered}
P I_{\text {trans }} \stackrel{k_{\text {trans }}-\text { cis }}{\rightleftharpoons} P I_{\text {cis }} \\
k_{\text {cis }- \text { trans }} \\
P I_{\text {trans }}^{0} \stackrel{k_{\text {flow }}}{\longrightarrow} P I_{\text {trans }} \stackrel{k_{\text {flow }}}{\longrightarrow} \emptyset \\
P I_{\text {cis }} \stackrel{\text { f }_{\text {flow }} \emptyset,}{\longrightarrow} \emptyset
\end{gathered}
$$

, where:

- PI $I_{\text {trans }}, P I_{\text {cis }} \rightarrow$ trans and cis photoinhibitors

- $k_{\text {trans-cis }}=k_{\text {cis-trans }}=10 \mathrm{~min}^{-1} \rightarrow$ photoswitching rates (note that these values were not determined experimentally and the value of 10 is used as a rough estimate)

- $k_{\text {flow }}=0.1 \mathrm{~min}^{-1} \rightarrow$ relative flowrate of the reactor

- $P I_{\text {trans }}^{0} \rightarrow$ initial trans-PI that is pumped into the reactor, where $\left[P I_{\text {trans }}^{0}\right]=$ const .

The total chymotrypsin concentration (bound and unbound forms) is constant in the reactor and is not modelled explicitly.

The outflow of the reactor is mixed with fluorogenic substrate $S u b$, and the following reactions have to be added to the system:

$$
\begin{gathered}
\text { Sub } \stackrel{V}{\rightarrow} A M C \\
S u b^{0} \stackrel{k_{\text {flow }}}{\longrightarrow} \text { Sub } \stackrel{k_{\text {flow }}}{\longrightarrow} \emptyset \\
A M C \stackrel{k_{\text {flow }}}{\longrightarrow} \emptyset
\end{gathered}
$$

, where:

- $A M C \rightarrow$ formed fluorescent product

- $V \rightarrow$ rate of enzymatic conversion according to Michaelis-Menten kinetics, taking competitive inhibition by the photoinhibitor in its cis and trans isomeric states into account:

$$
V=\frac{k_{\text {cat }}[\mathrm{Cr}] \cdot \frac{[\text { Sub }]}{K_{M}}}{1+\frac{[\text { Sub] }]}{K_{M}}+\frac{\left[P I_{\text {trans }}\right]}{K_{\text {trans }}}+\frac{\left[P I_{\text {cis }}\right]}{K_{\text {cis }}}}
$$


The model is more sensitive to light pulses than observed throughout the performance of experiments. For example, the model is able to detect short transient pulses of AMC (in the seconds range), that are not observed experimentally. Thus, to take experimental conditions into account, we resample the output of the model with a rolling average of 60 second.

A detailed explanation on how to use the model and which parameters can be altered is given along the Python script, which can be accessed using the Github link from above. 


\section{References}

1) Priesch, B.; Rück-Braun, K. Efficient Preparation of Nitrosoarenes for the Synthesis of Azobenzenes. J. Org. Chem. 2005, 70, 2350-2352.

2) Cheng, S.-C.; Chen, K.-J.; Suzaki, Y.; Tsuchido, Y.; Kuo, T.-S.; Osakada, K.; Horie, M. Reversible LaserInduced Bending of Pseudorotaxane Crystals. J. Am. Chem. Soc. 2018, 140, 90-93.

3) Westphal, M. V.; Schafroth, M. A.; Sarott, R. C.; Imhof, M. A.; Bold, C. P.; Leippe, P.; Dhopeshwarkar, A.; Grandner, J. M.; Katritch, V.; Mackie, K.; Trauner, D.; Carreira, E. M.; Frank, J. A. Synthesis of Photoswitchable $\Delta$ 9-Tetrahydrocannabinol Derivatives Enables Optical Control of Cannabinoid Receptor 1 Signaling. J. Am. Chem. Soc. 2017, 139, 18206-18212.

4) Kano, N.; Yoshino, J.; Kawashima, T. Photoswitching of the Lewis Acidity of a Catecholborane Bearing an Azo Group Based on the Change in Coordination Number of Boron. Org. Lett. 2005, 7, 3909-3911. 


\section{NMR Spectra}

NOTE: Due to the trans/cis isomerization of some compounds containing an azobenzene functionality, more signals were observed in the ${ }^{1} \mathrm{H}$ and ${ }^{13} \mathrm{C}$ spectra than would be expected for the pure trans-isomer. Only signals for the major trans-isomer are reported.

(E)-1-phenyl-2-(3-(trifluoro-14-boraneyl)phenyl)diazene, potassium salt (1)
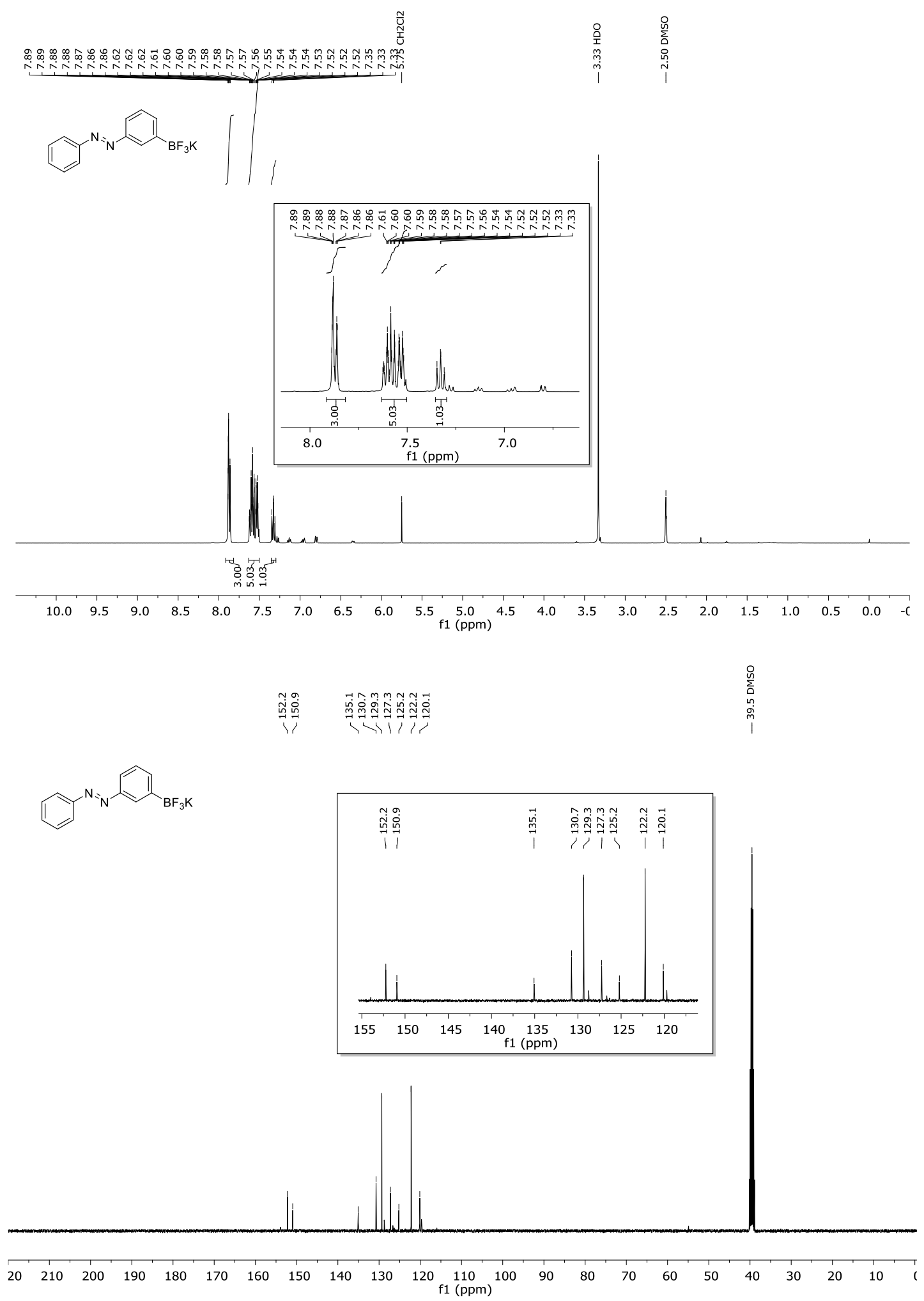


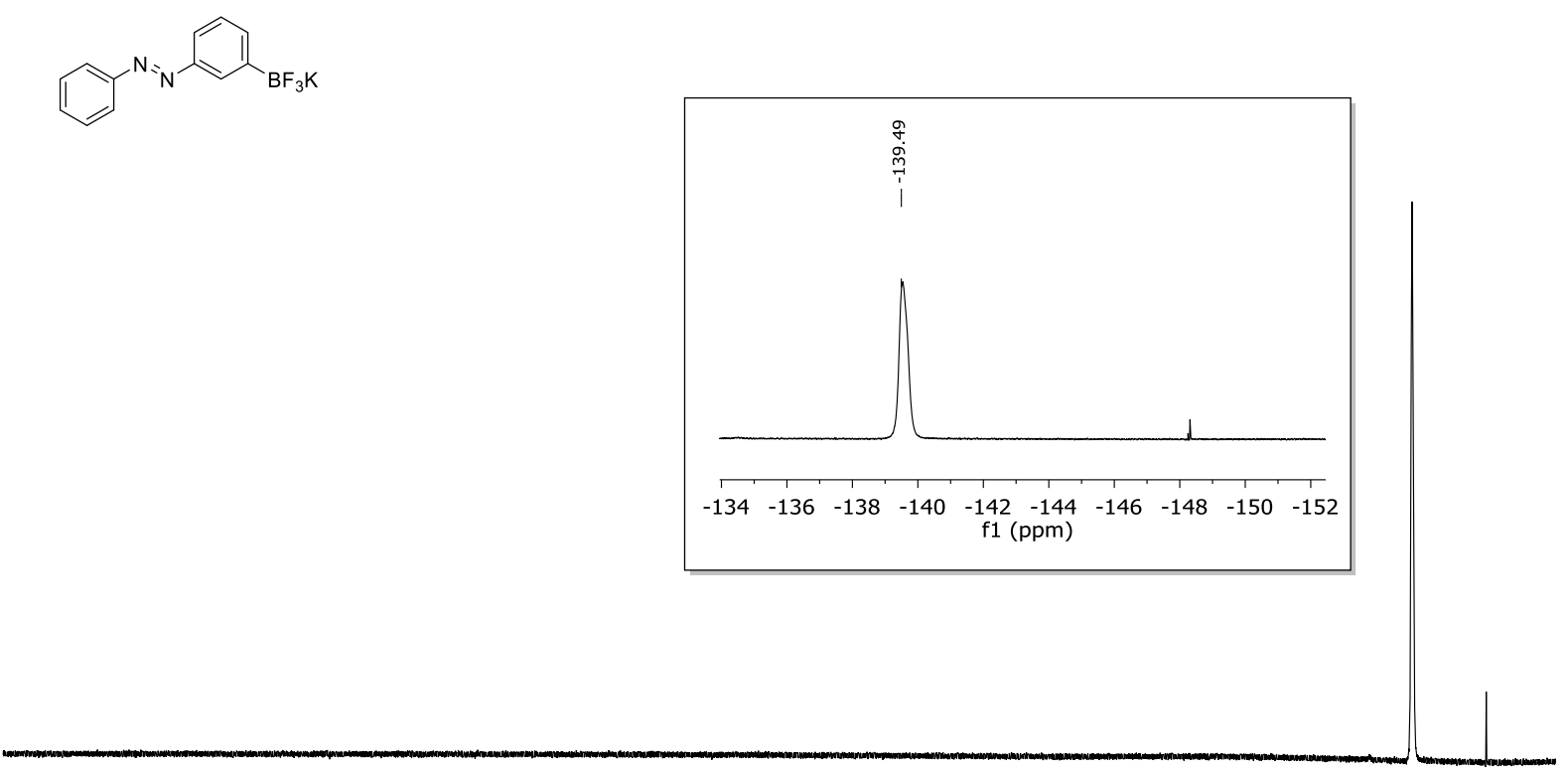

\begin{tabular}{|c|c|c|c|c|c|c|c|c|c|c|c|c|c|c|c|c|}
\hline 20 & 10 & 0 & -10 & -20 & -30 & -40 & -50 & $\begin{array}{c}-60 \quad-70 \\
\mathrm{f} 1(\mathrm{ppm})\end{array}$ & -80 & -90 & -100 & -110 & $\begin{array}{c}-120 \\
\underset{N}{N} \\
1\end{array}$ & -130 & -140 & -150 \\
\hline
\end{tabular}

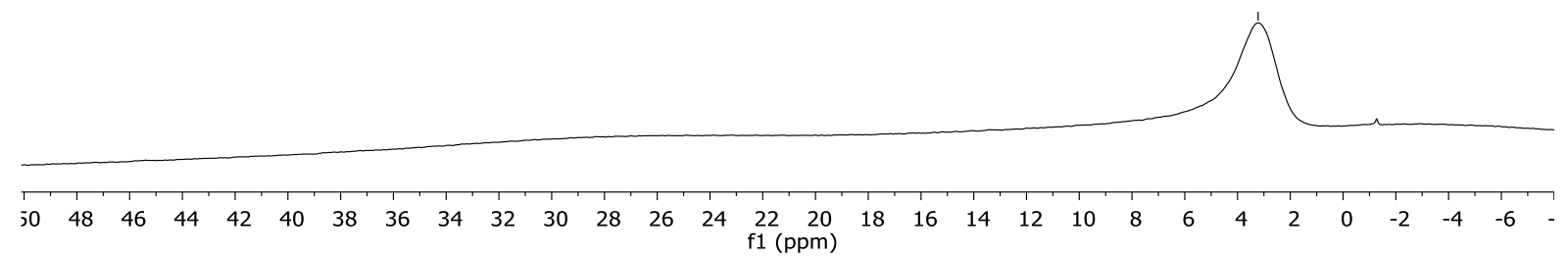


(E)-1-phenyl-2-(3-(trifluoro-14-boraneyl)phenyl)diazene, potassium salt (2)
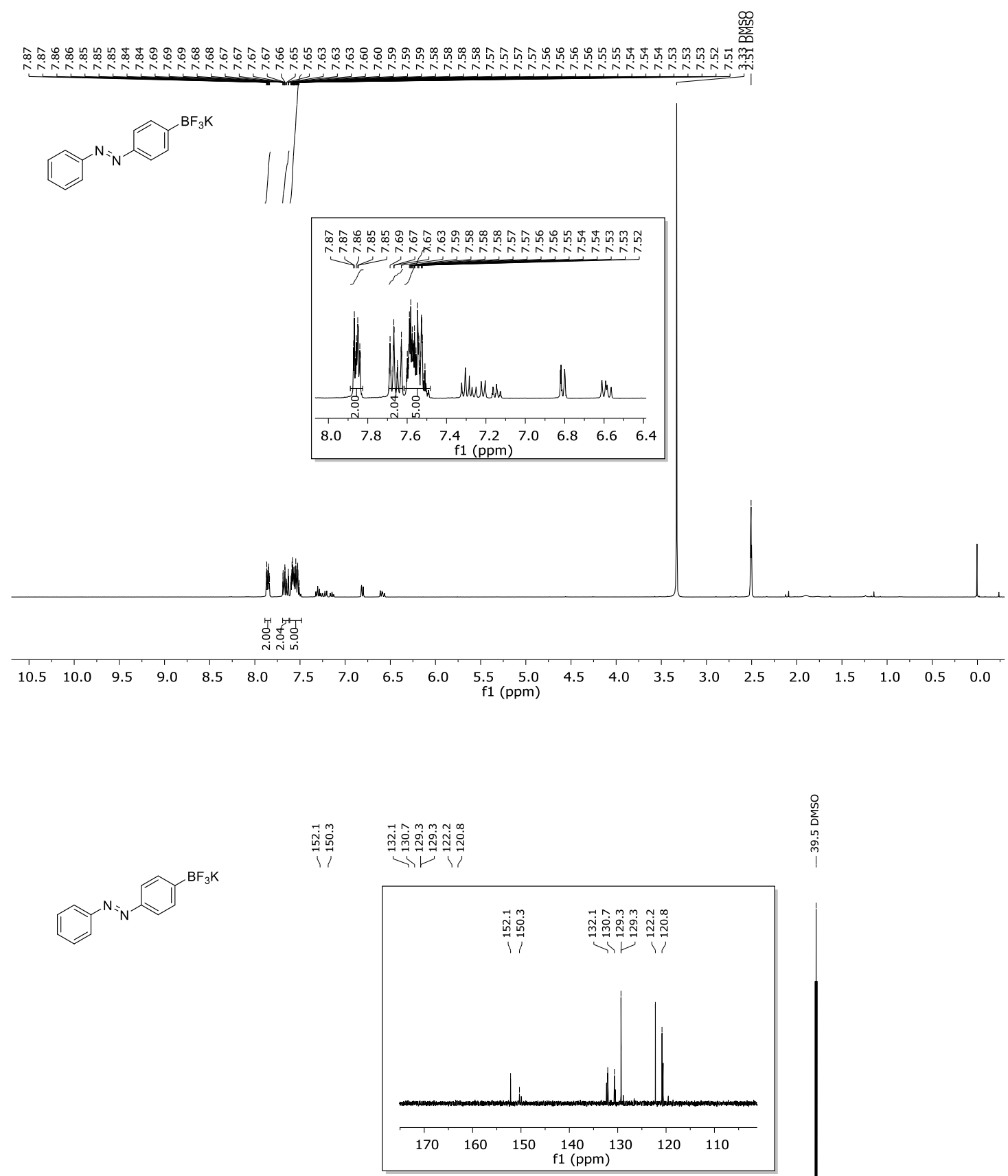

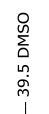
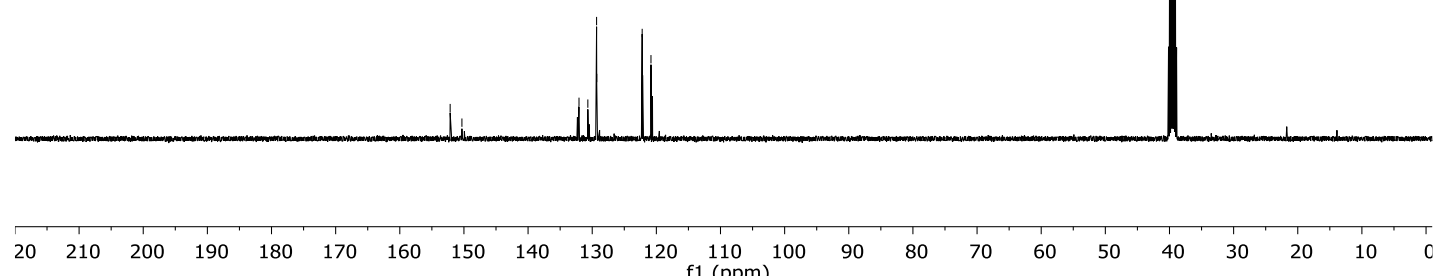
$y^{N^{N}}$

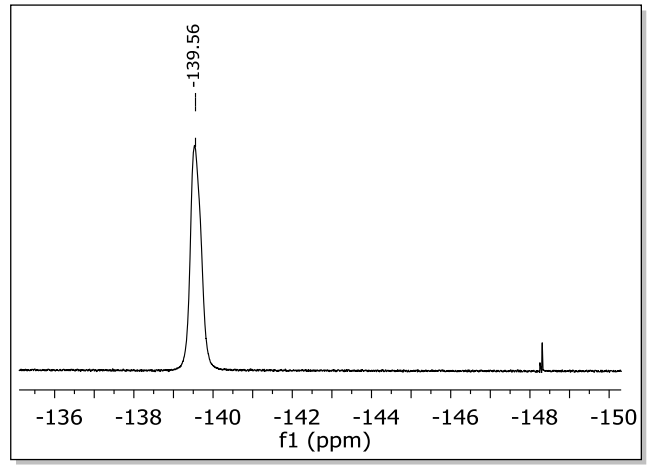

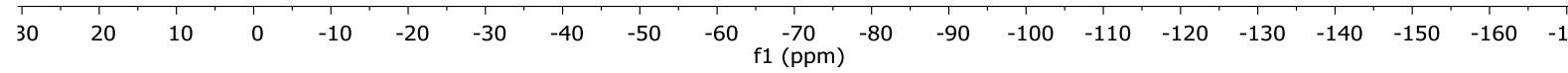

$y^{\mathrm{N}_{\mathrm{N}}}$

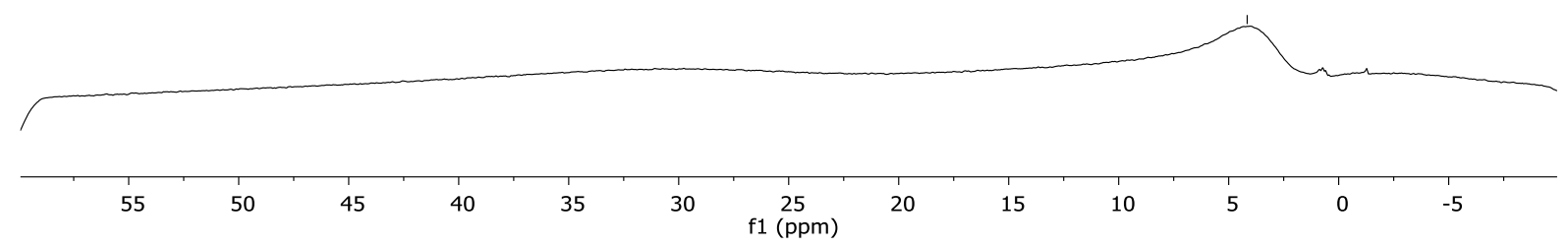




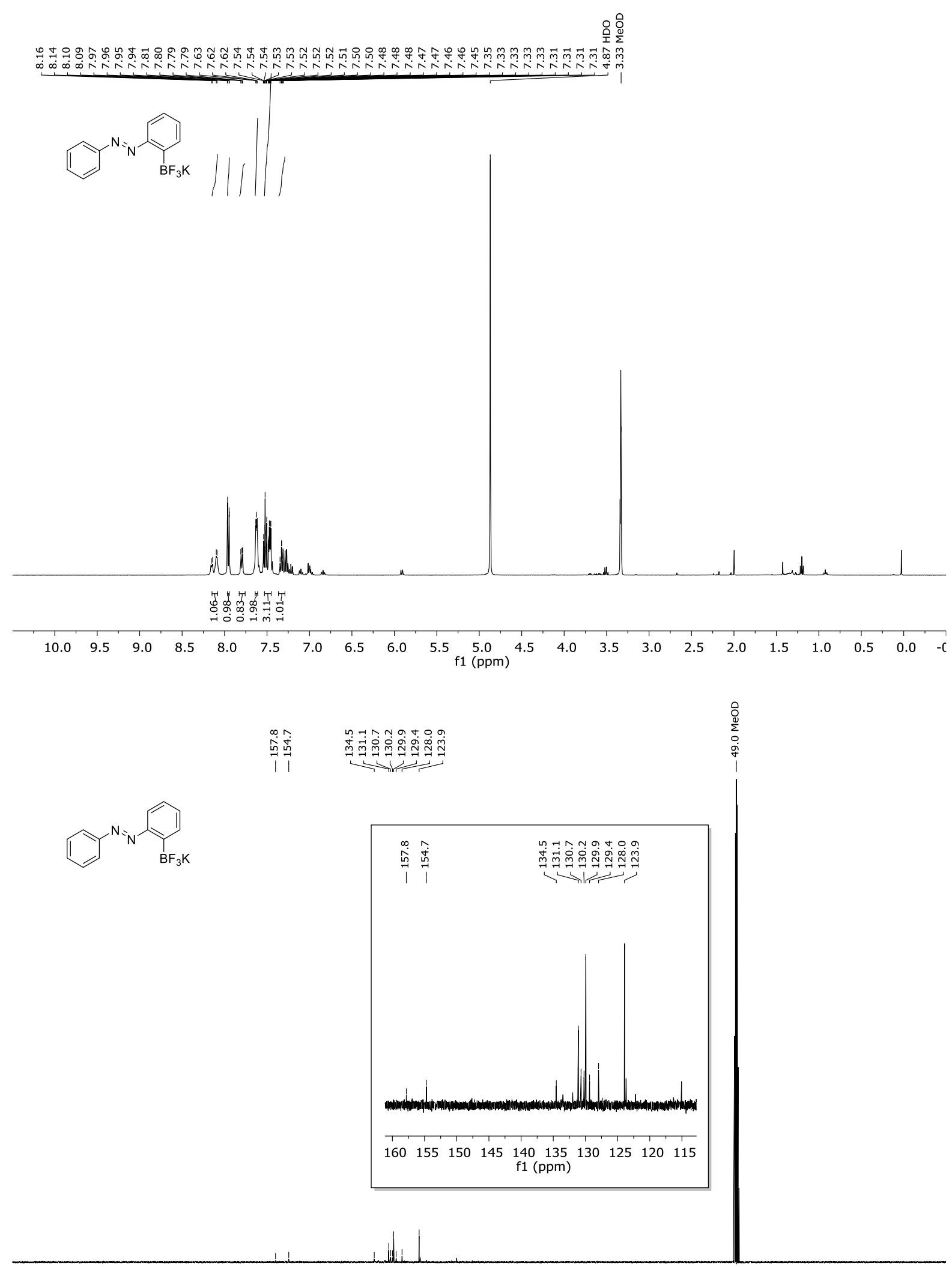

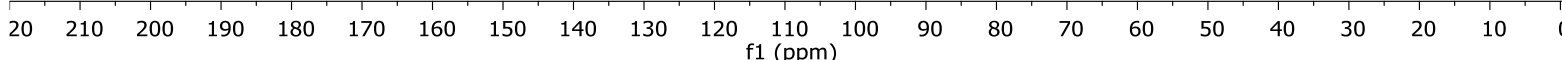


$\mathrm{CO}_{\mathrm{BF} \mathrm{F}_{3} \mathrm{~K}}^{\mathrm{N}}$

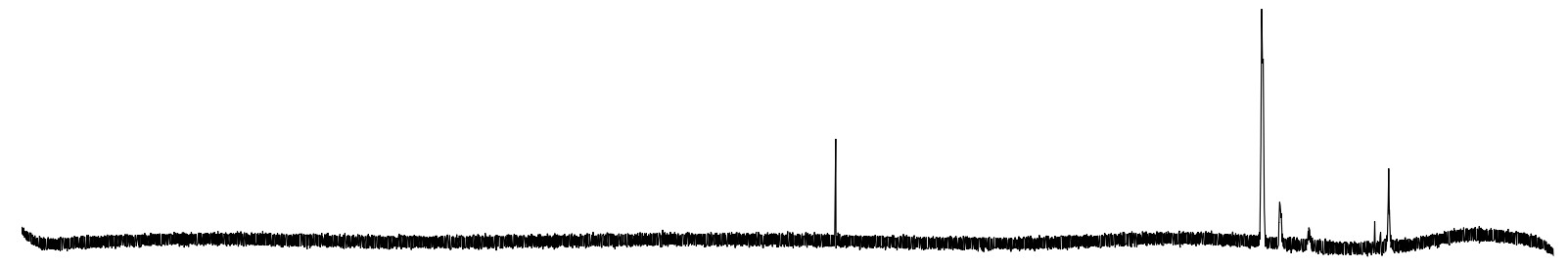

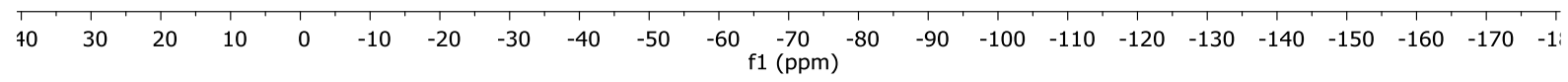

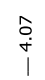<smiles>[Y]c1ccccc1N=Nc1ccccc1</smiles>

$\begin{array}{llllllllllllllllllllllllllllllllllllllllllllllll}52 & 50 & 48 & 46 & 44 & 42 & 40 & 38 & 36 & 34 & 32 & 30 & 28 & 26 & 24 & 22 & 20 & 18 & 16 & 14 & 12 & 10 & 8 & 6 & 4 & 2 & 0 & -2 & -4\end{array}$ f1 (ppm) 
<smiles>COc1ccccc1NNc1cccc(Br)c1</smiles>

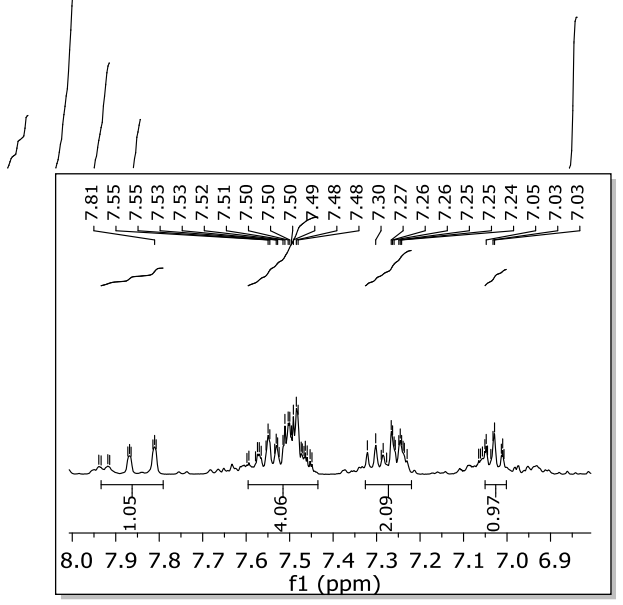

whe

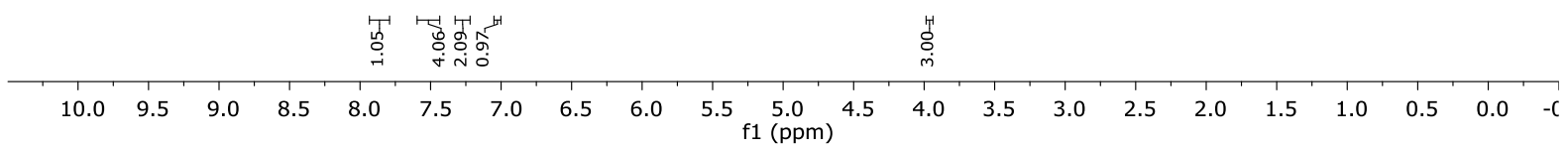

苟㔯

นุ่

$\mathrm{N}_{\mathrm{BF}_{3} \mathrm{~K}}$
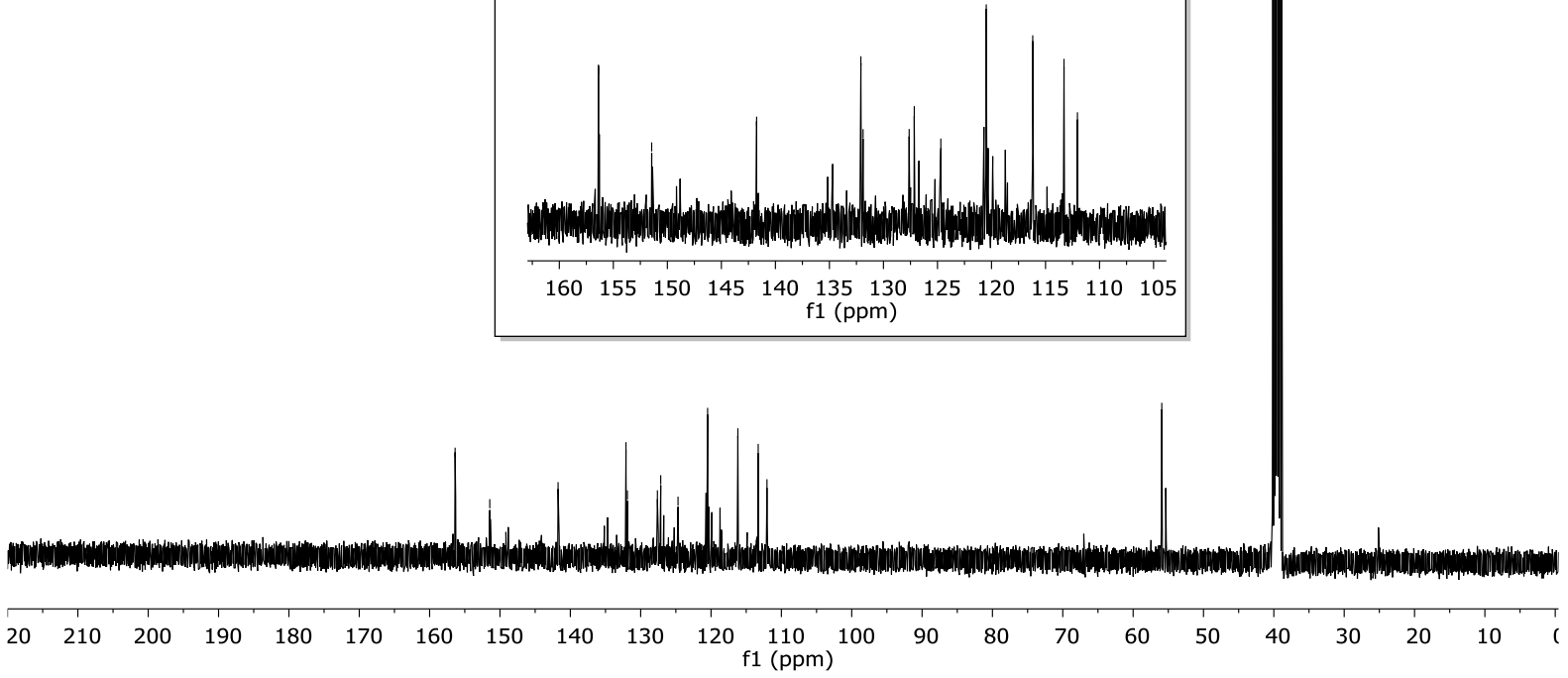

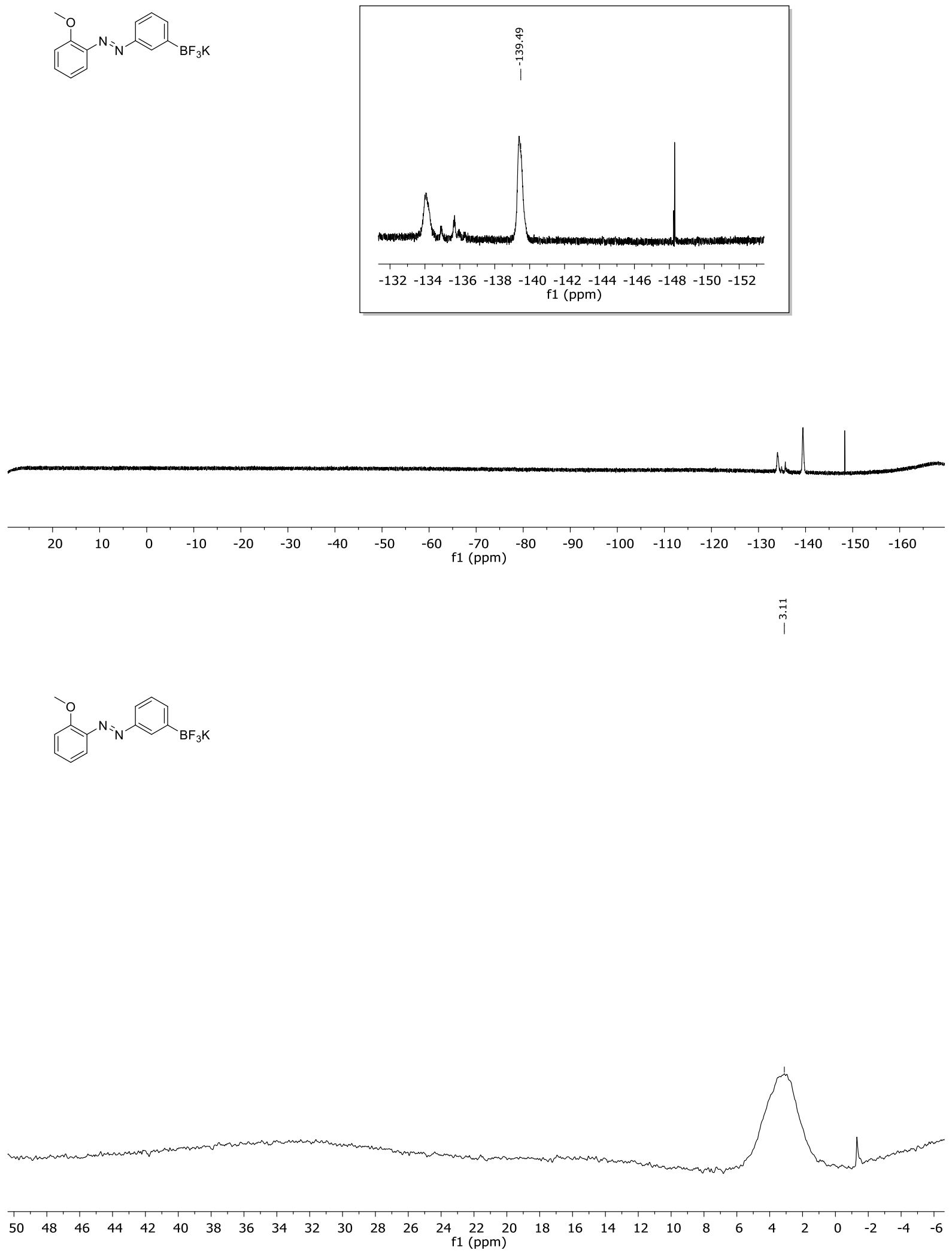

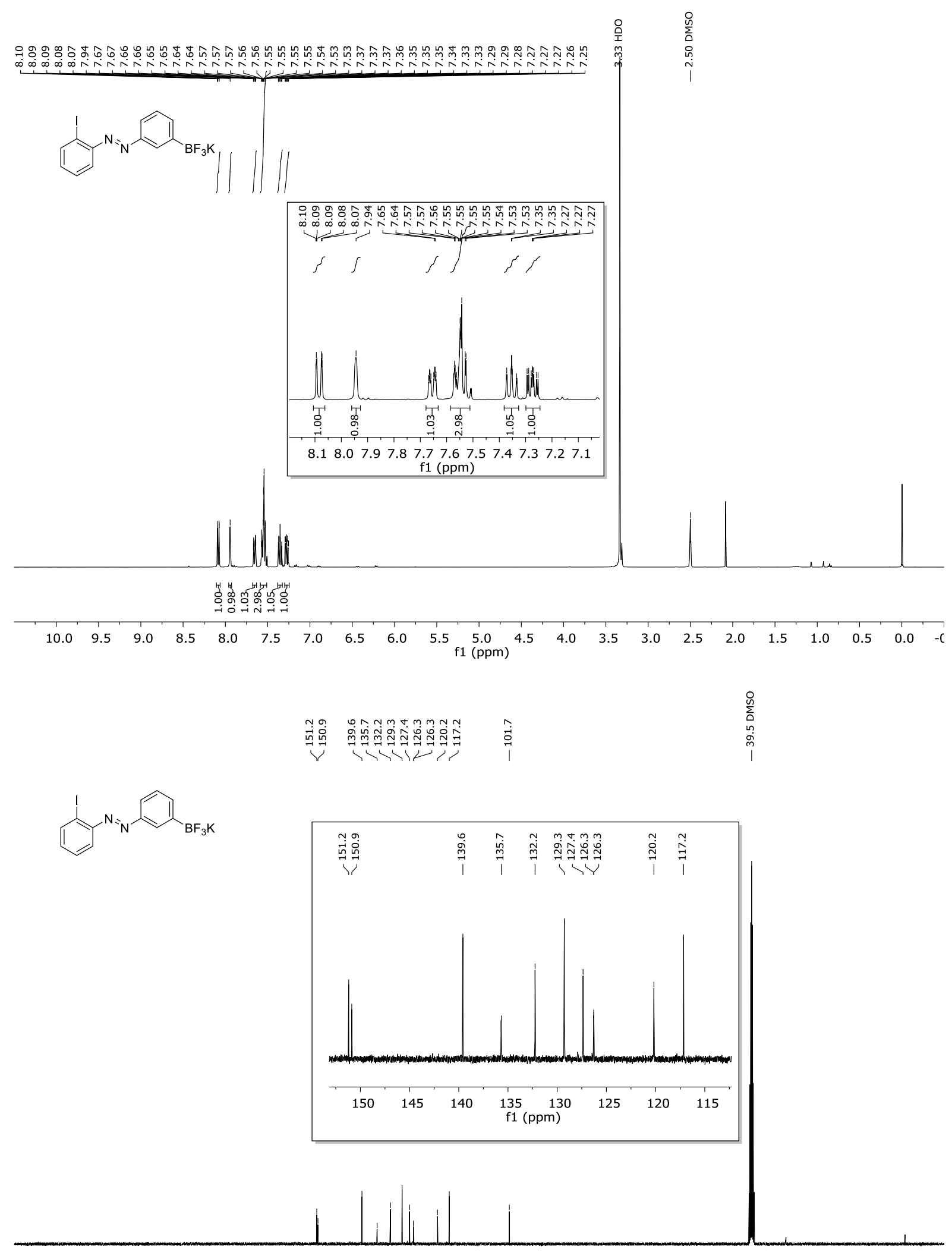

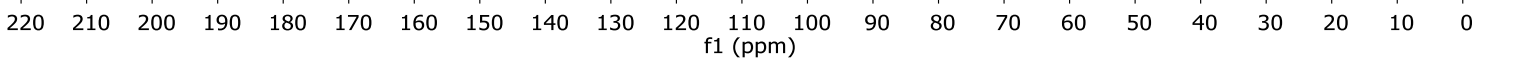


in
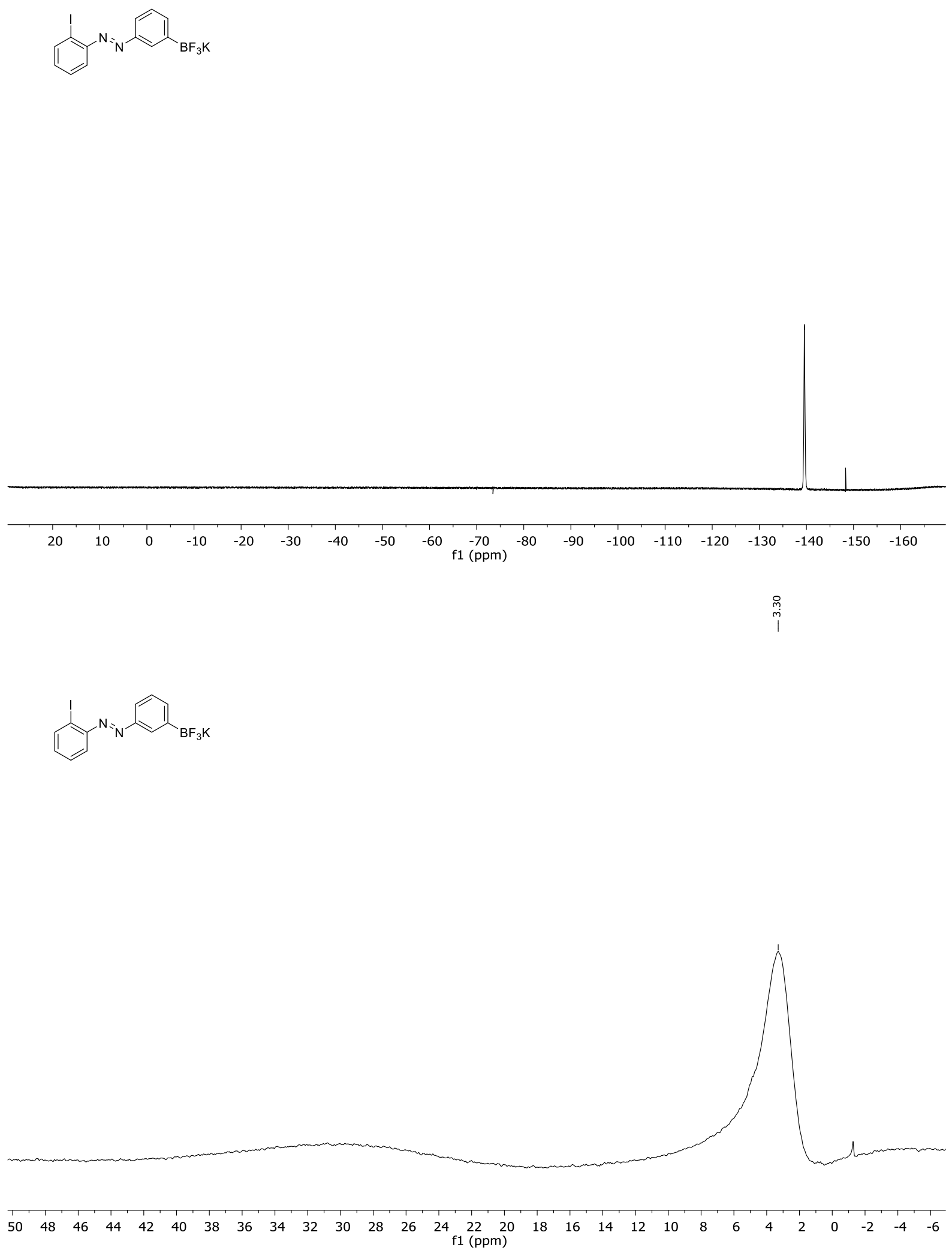

- S122 - 
(E)-1-(2,6-difluorophenyl)-2-(3-(trifluoro-14-boraneyl)phenyl)diazene, potassium salt (6)

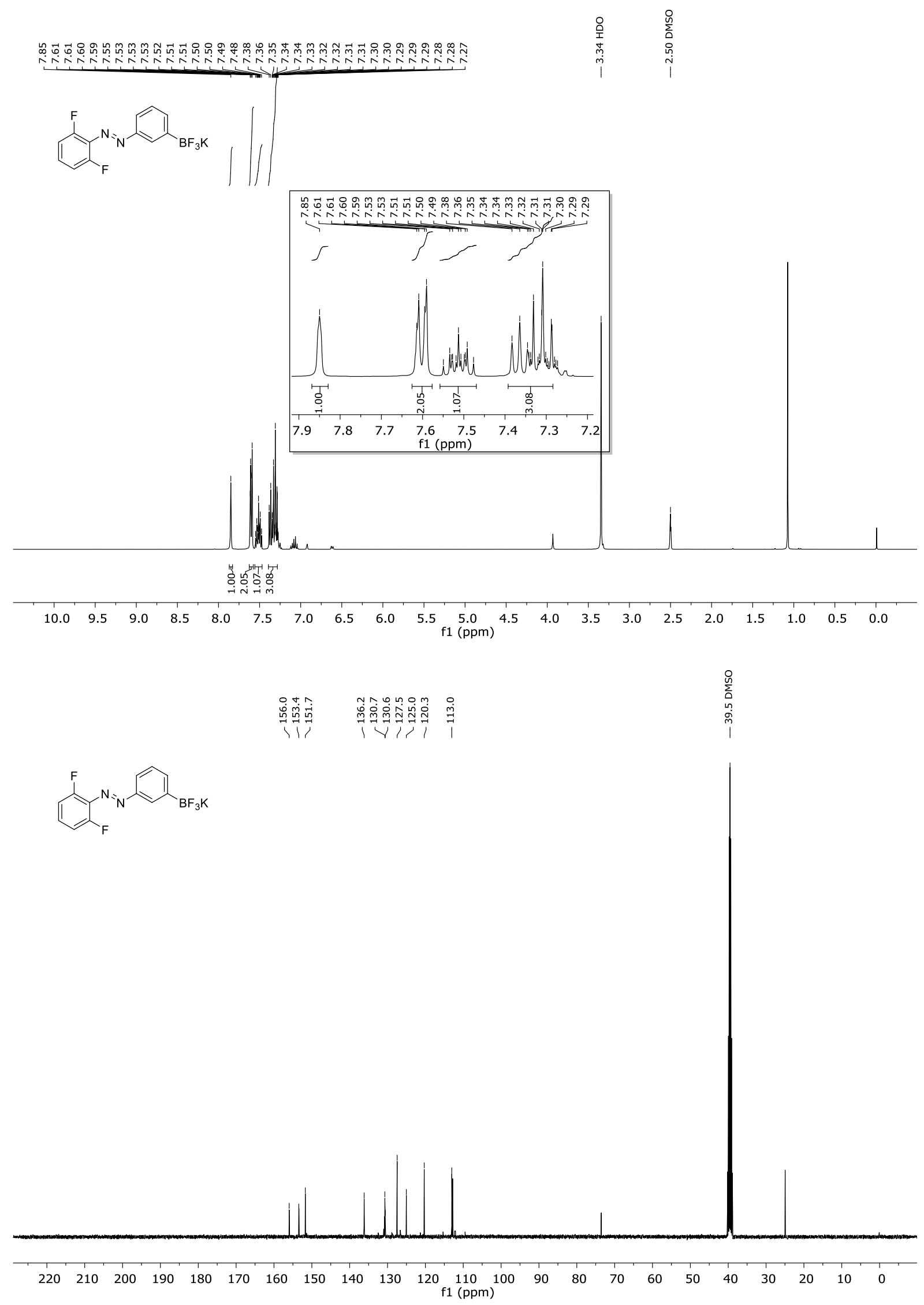



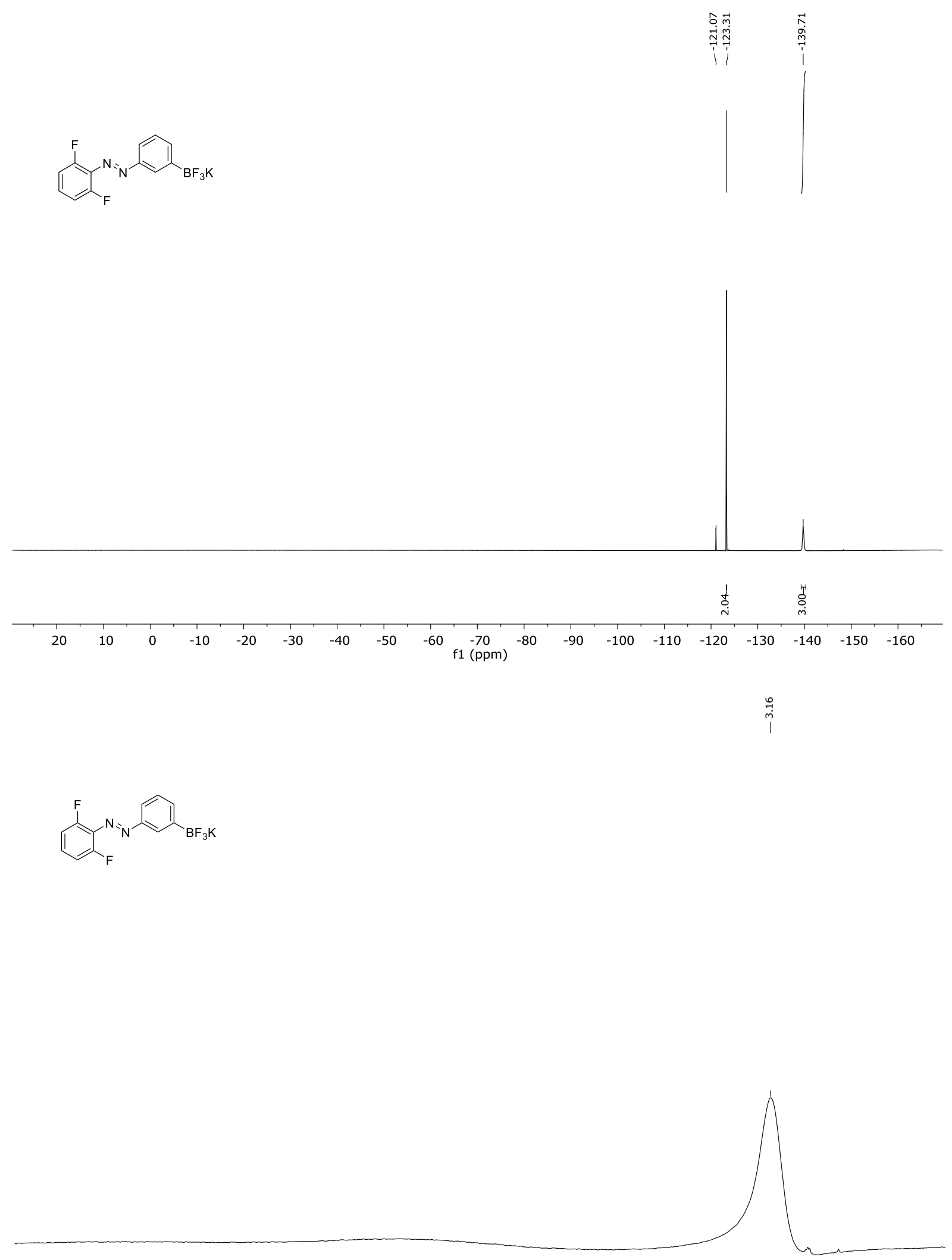

$\begin{array}{lllllllllllllllllllllllllllllllllllllllllll}52 & 50 & 48 & 46 & 44 & 42 & 40 & 38 & 36 & 34 & 32 & 30 & 28 & 26 & 24 & 22 & 20 & 18 & 16 & 14 & 12 & 10 & 8 & 6 & 4 & 2 & 0 & -2 & -4 & -6 & -i\end{array}$ 
(E)-1-(2-isopropylphenyl)-2-(3-(trifluoro-14-boraneyl)phenyl)diazene, potassium salt (7)

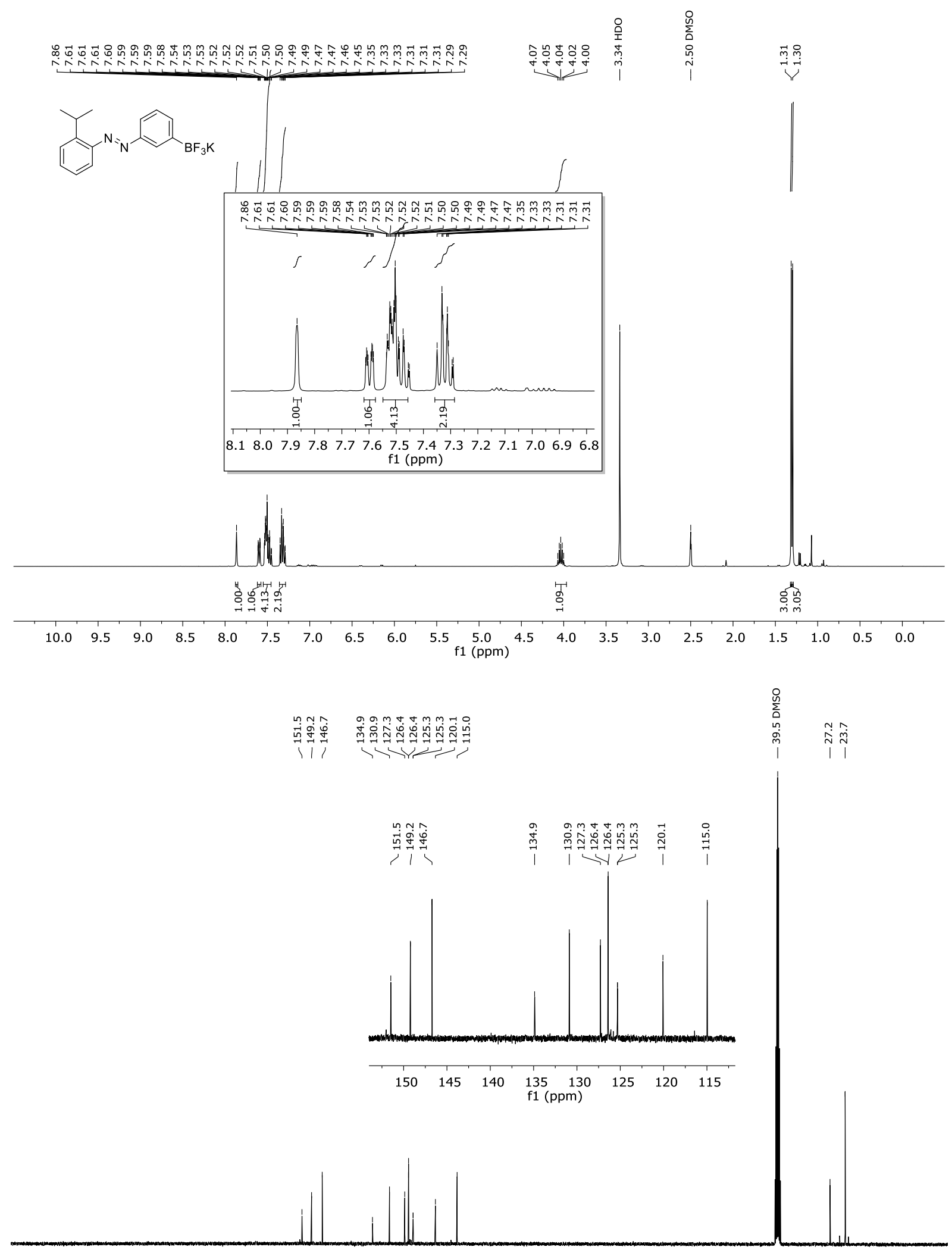

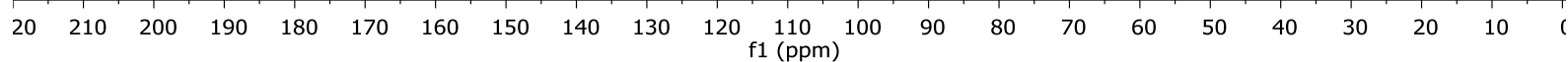


ờ
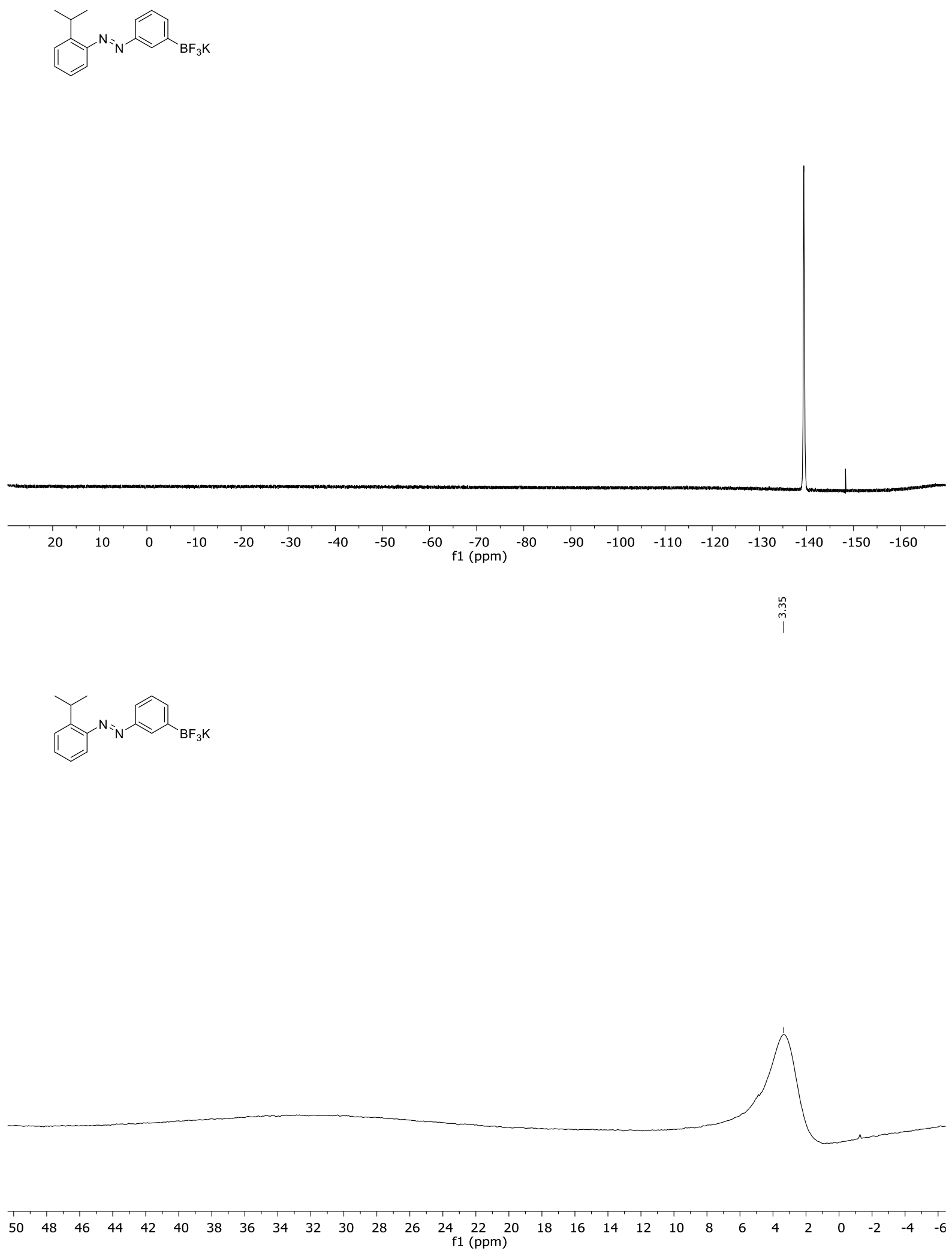

- S126 - 
(E)-1-(o-tolyl)-2-(3-(trifluoro-14-boraneyl)phenyl)diazene, potassium salt (8)

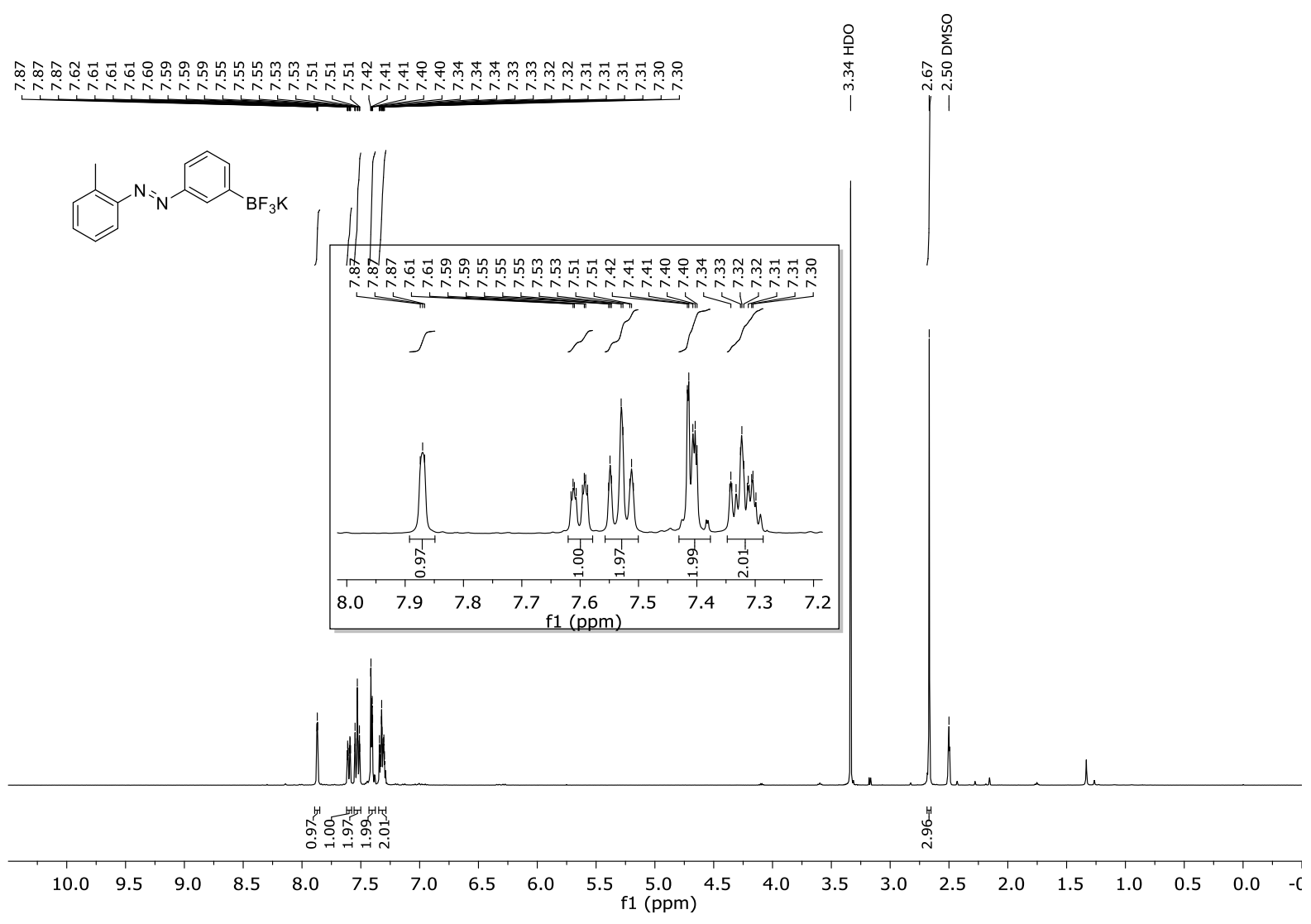

至兽

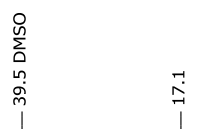

$\mathrm{N}^{\mathrm{N}_{\mathrm{N}}} \mathrm{BF}_{3 \mathrm{~K}}$
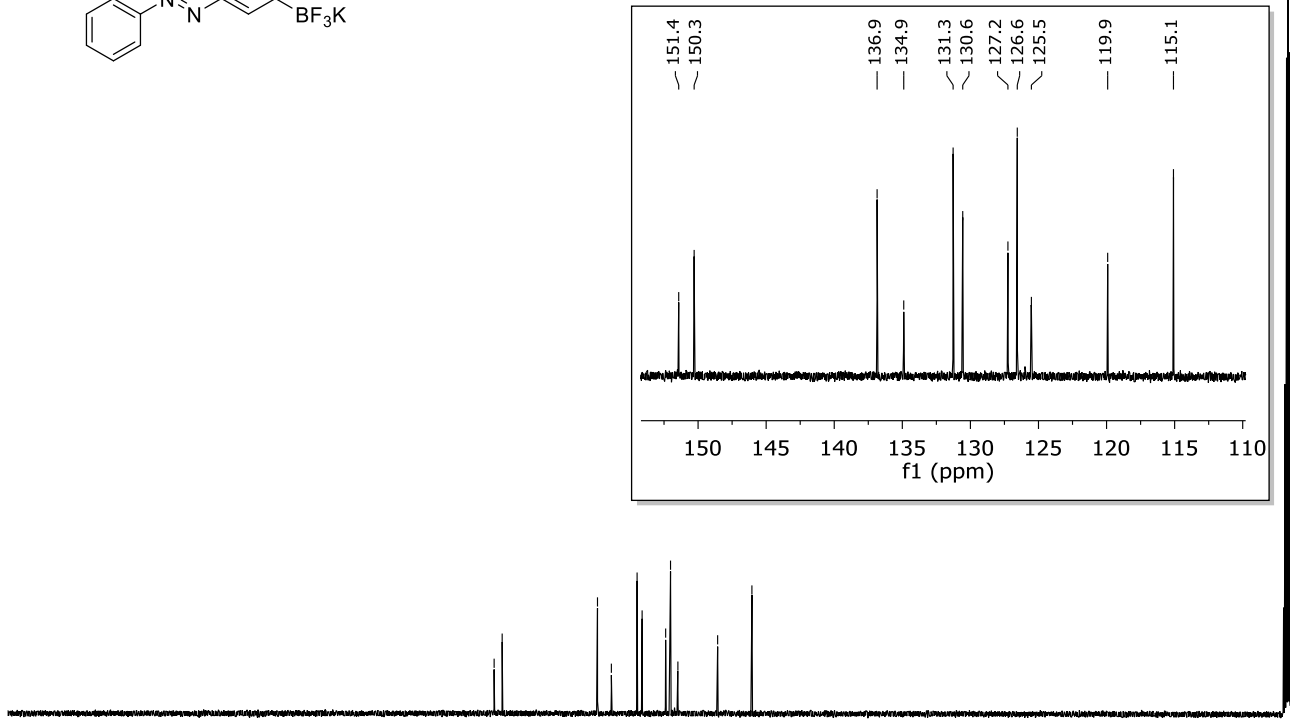

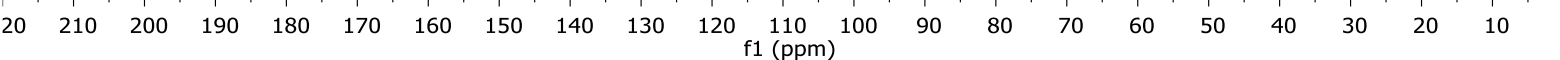




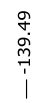
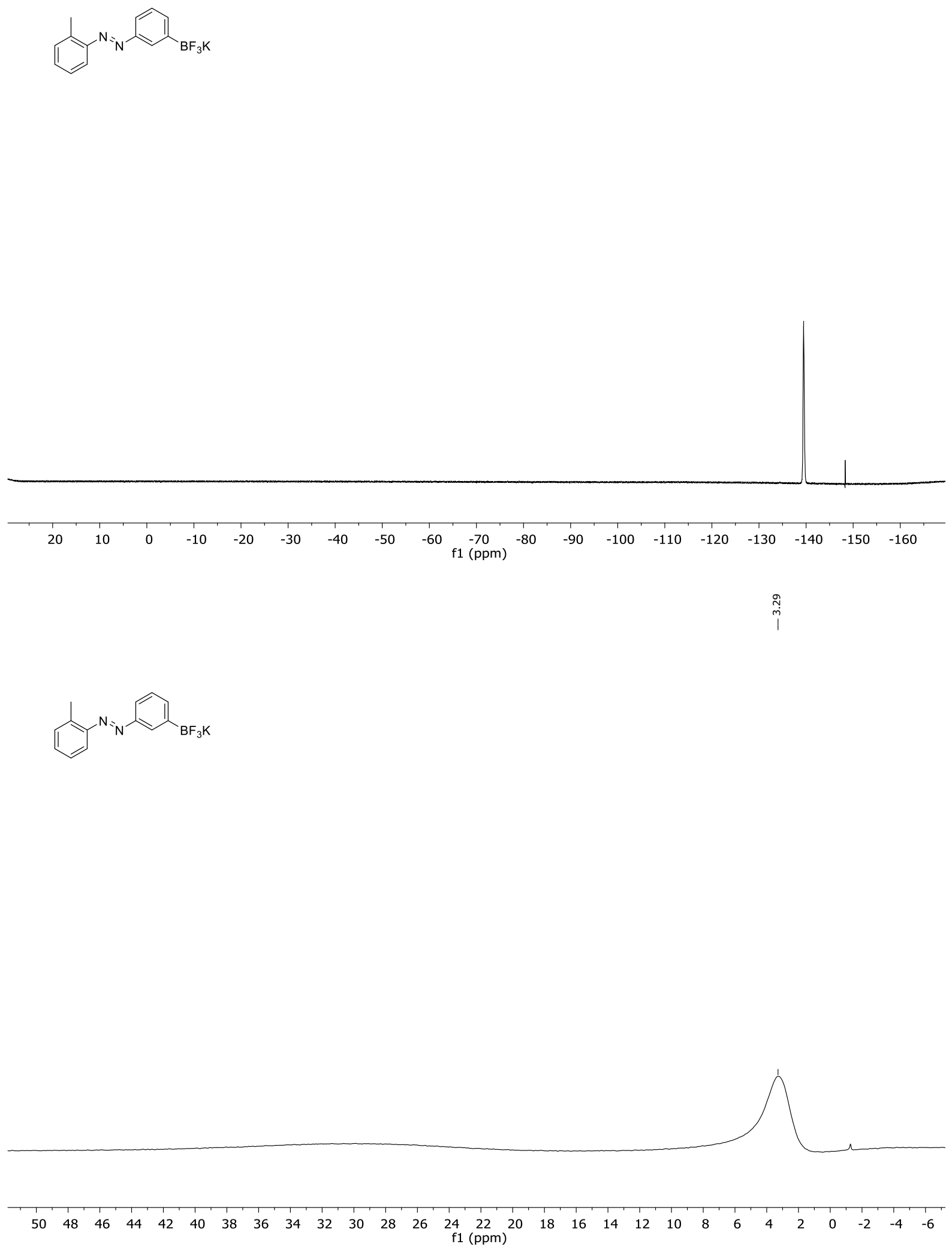

- S128 - 
(E)-1-(m-tolyl)-2-(3-(trifluoro-14-boraneyl)phenyl)diazene, potassium salt (9)
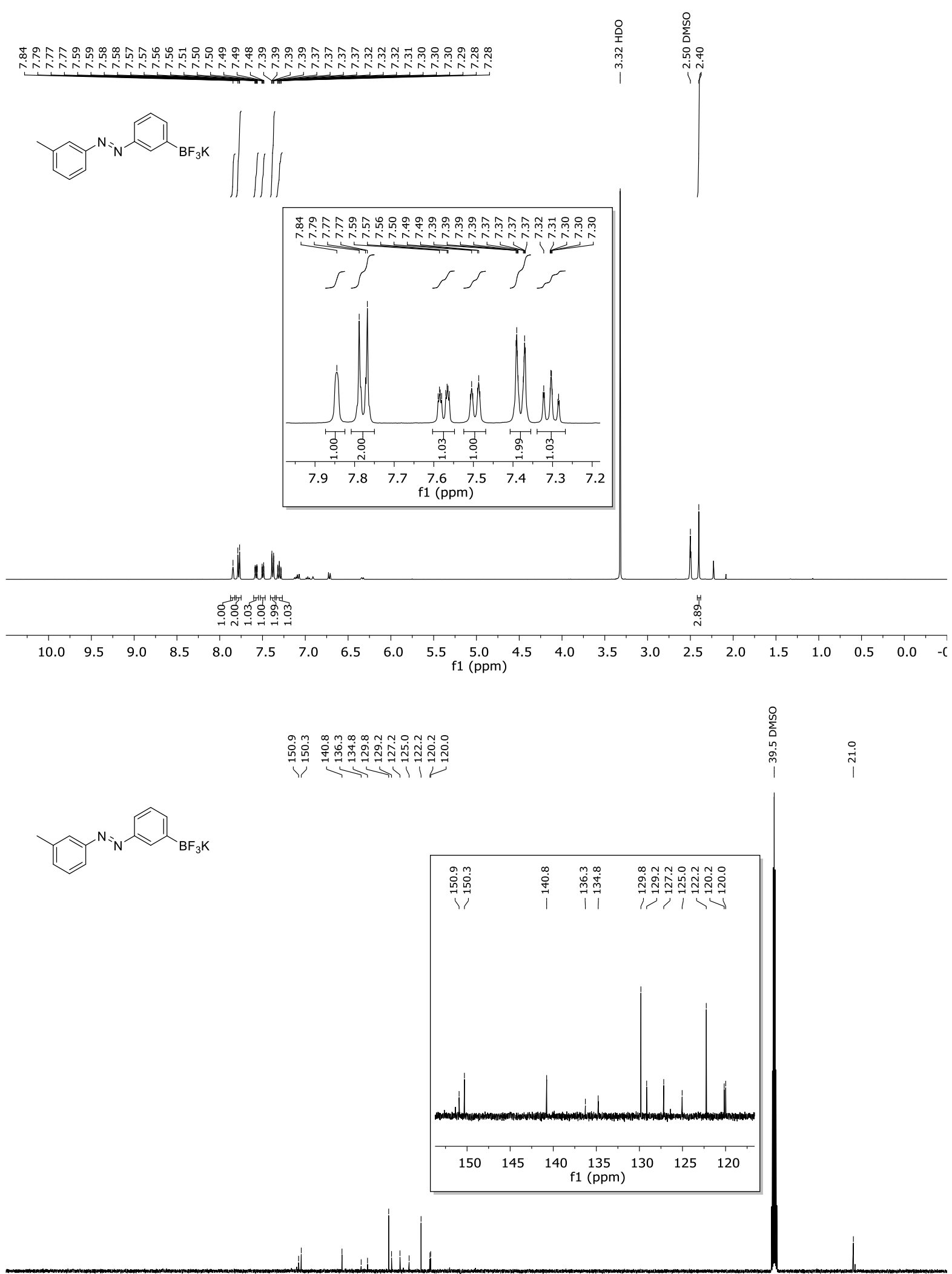

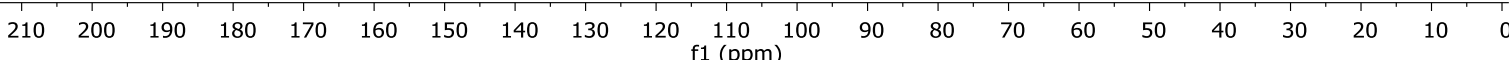




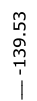
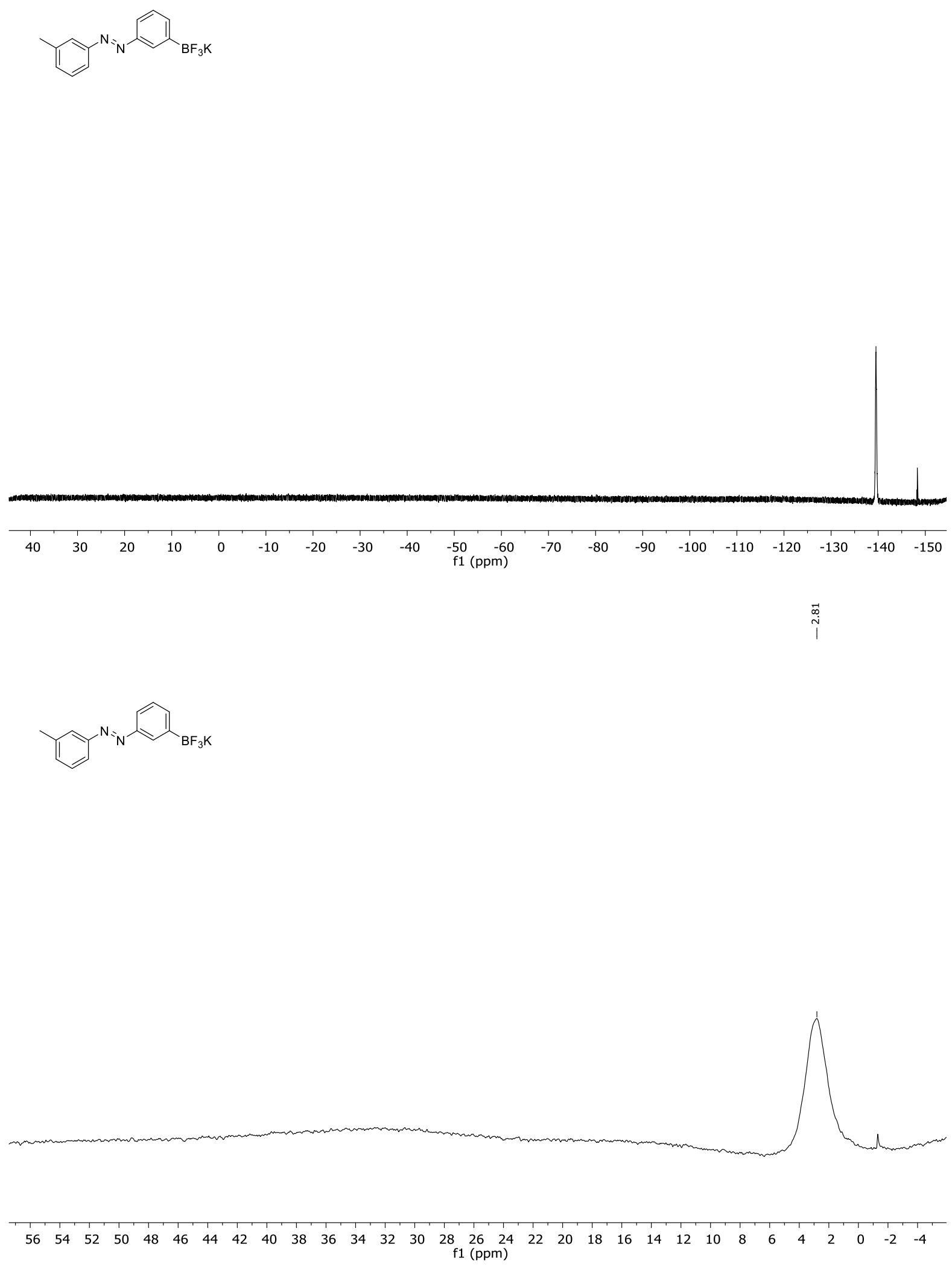

- S130 - 
(E)-1-(3,5-dimethylphenyl)-2-(3-(trifluoro-l4-boraneyl)phenyl)diazene, potassium salt (10)

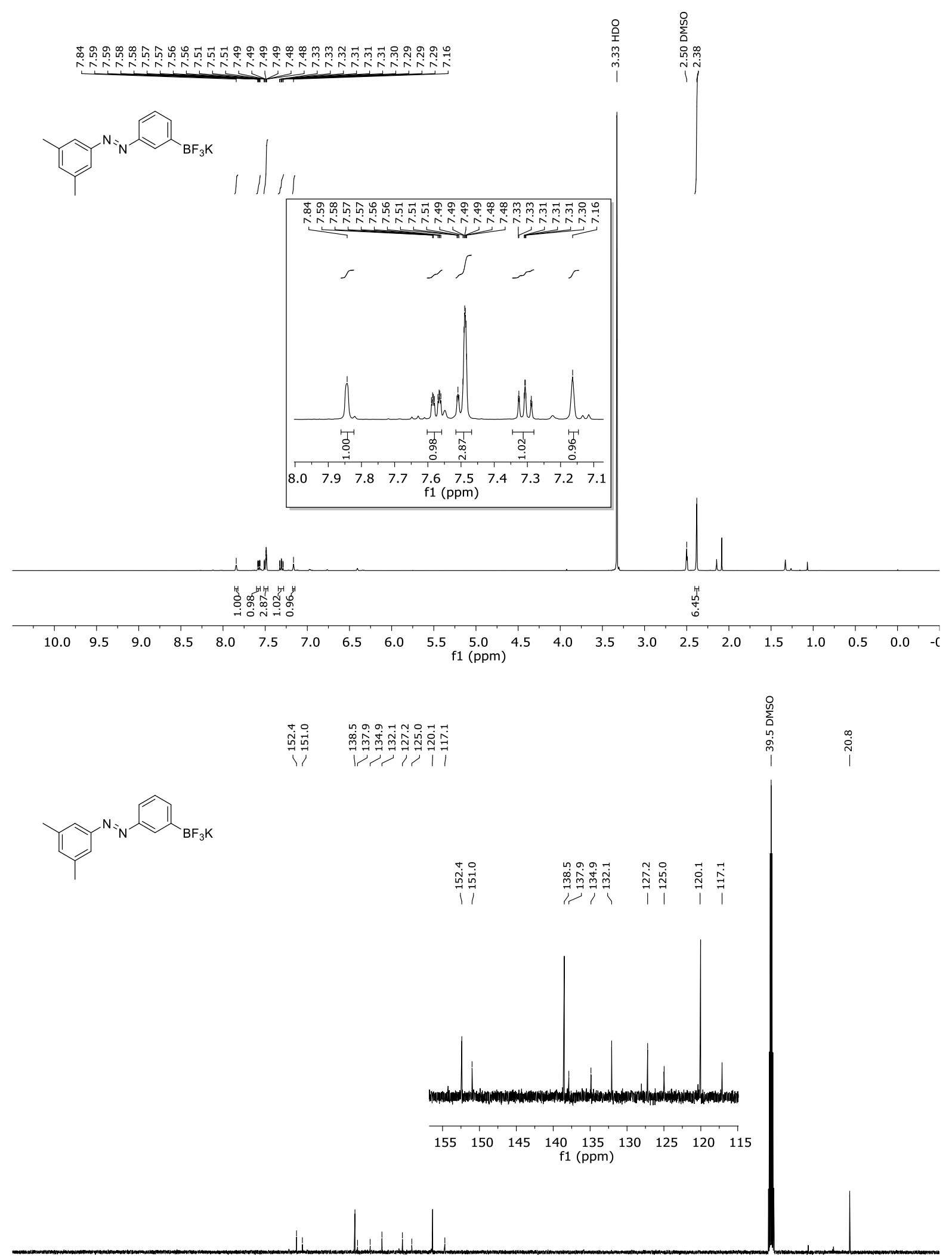

\begin{tabular}{llllllllllllllllllllllll}
\hline 20 & 210 & 200 & 190 & 180 & 170 & 160 & 150 & 140 & 130 & 120 & 110 & 100 & 90 & 80 & 70 & 60 & 50 & 40 & 30 & 20 & 10 & (
\end{tabular} 


$$
\mathrm{C}_{\mathrm{BF}_{3} \mathrm{~K}}
$$

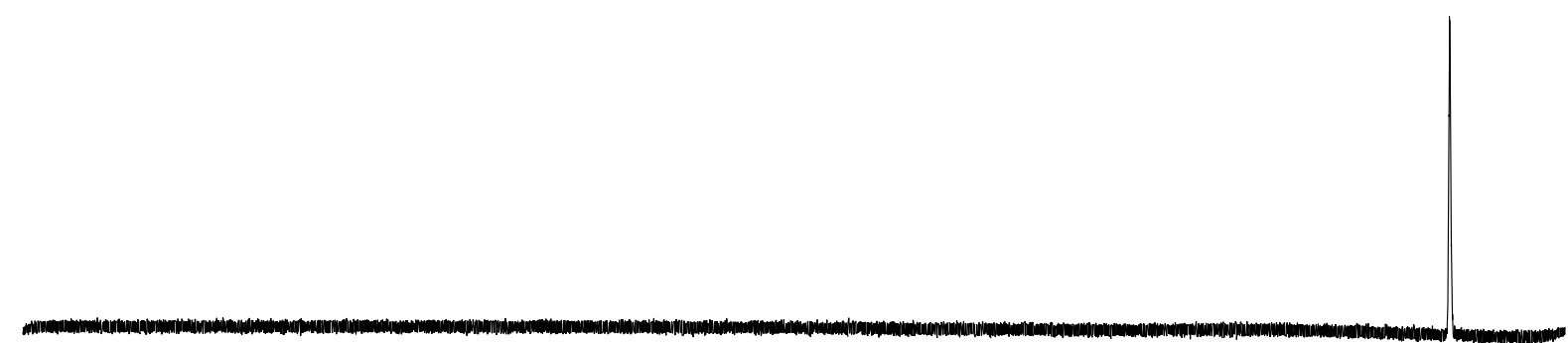<smiles>Cc1cc(C)cc(N=Nc2cccc([B-]C([N+])(F)F)c2)c1</smiles>

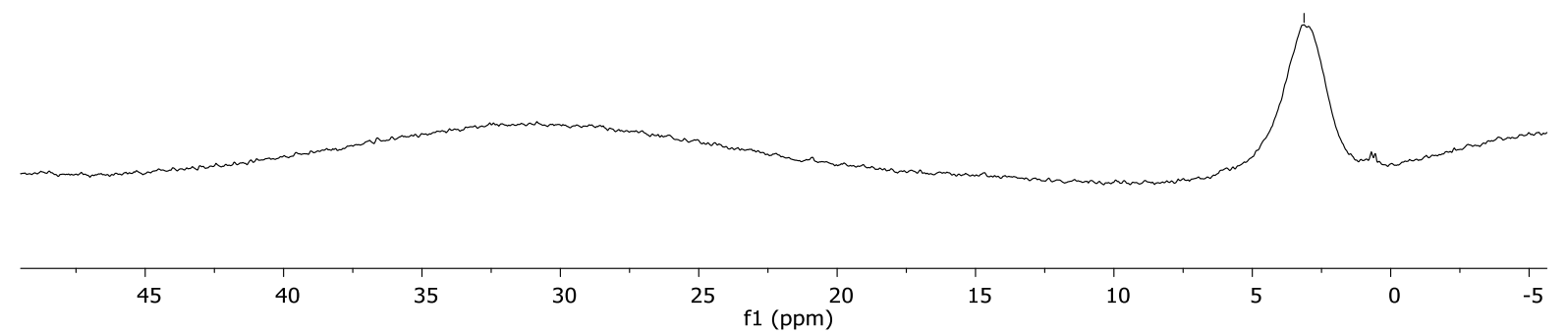




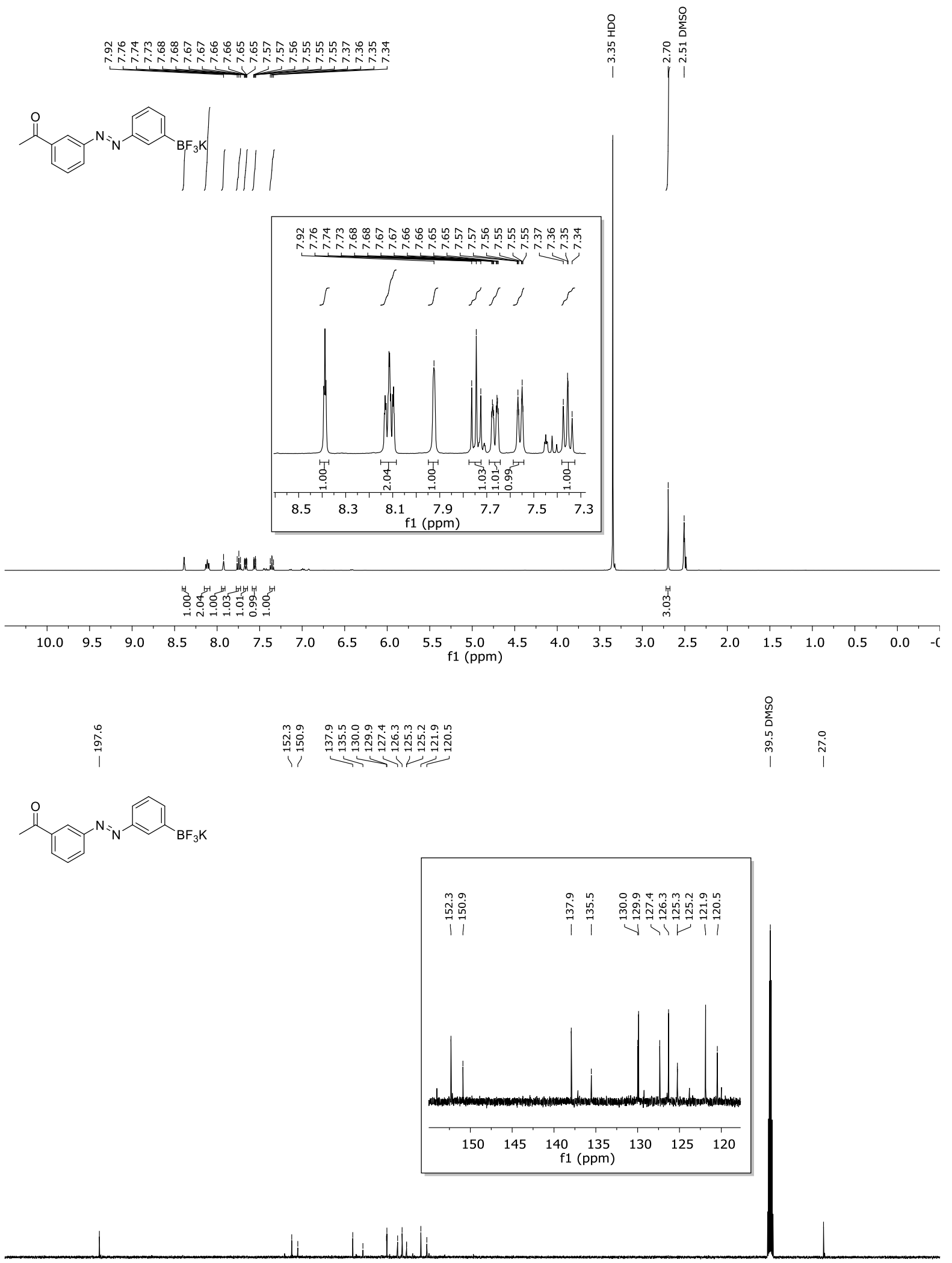

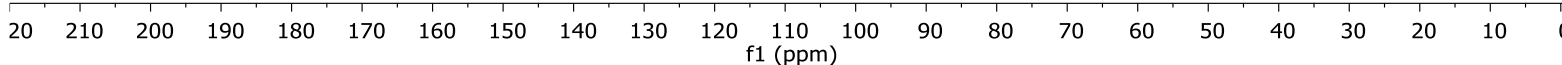



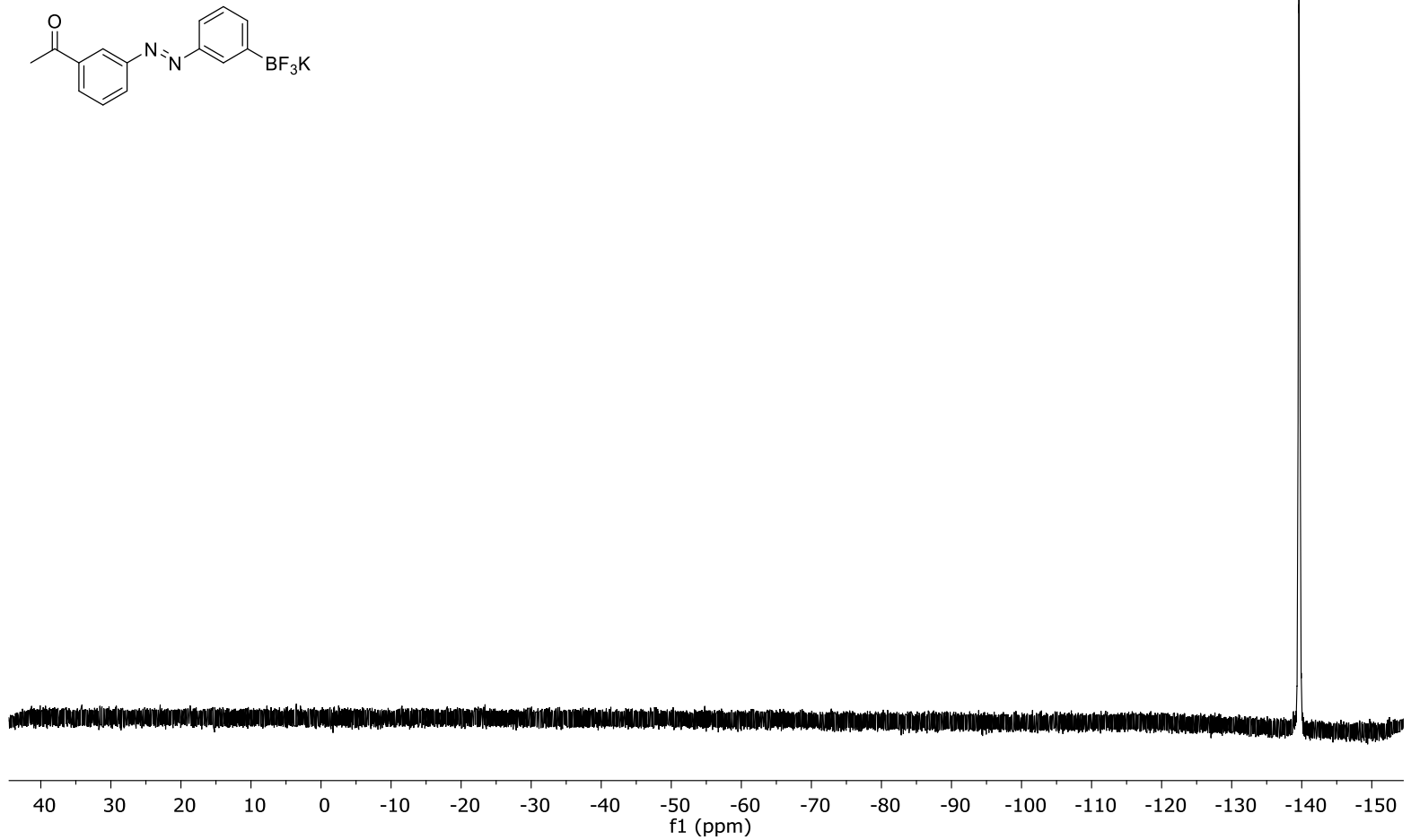

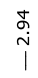
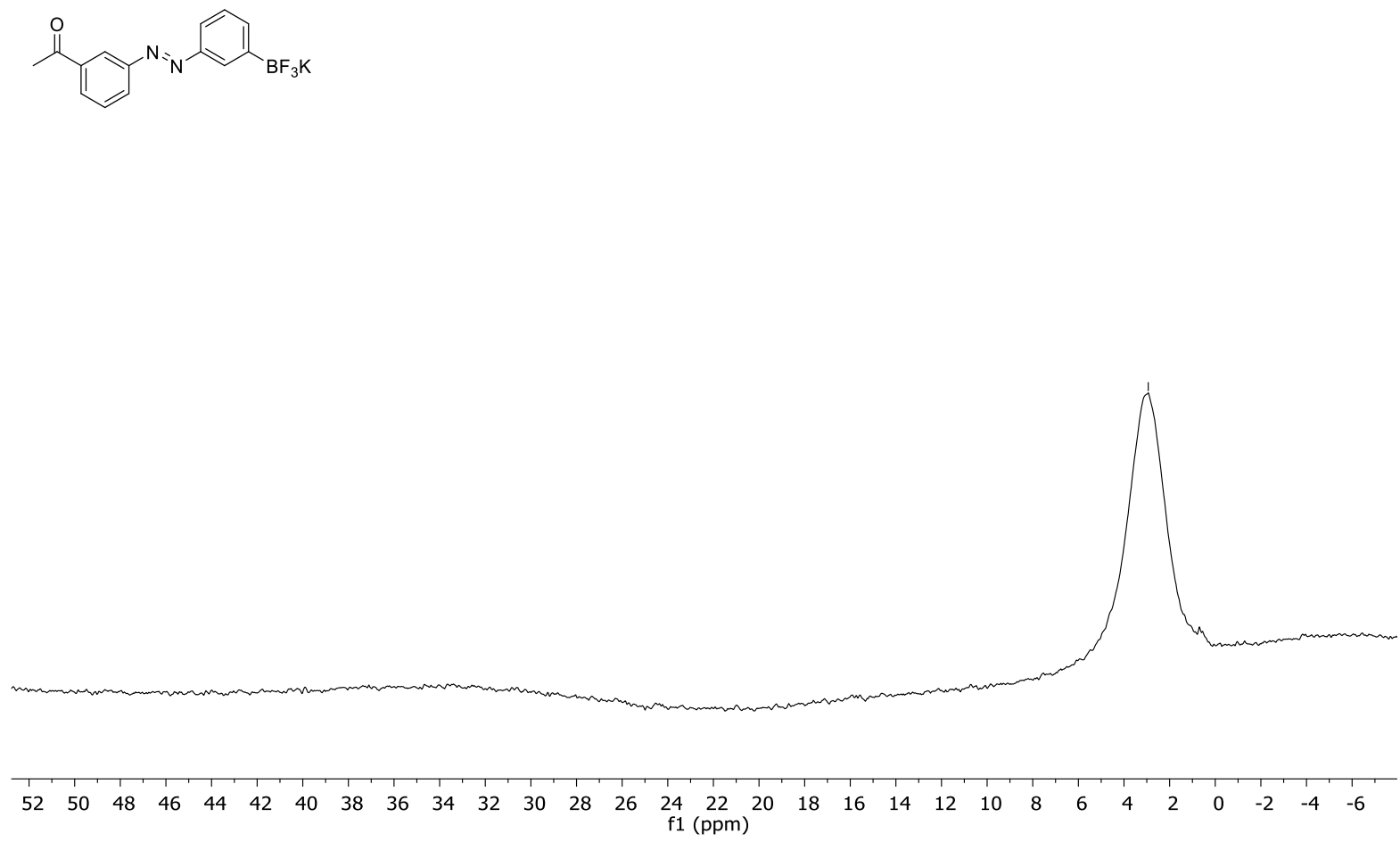

- S134 - 
œ

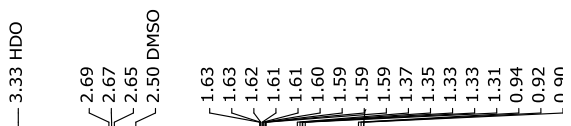

${ }_{B_{\mathrm{Bu}}} \mathrm{N}_{\mathrm{N}^{\mathrm{N}}}$

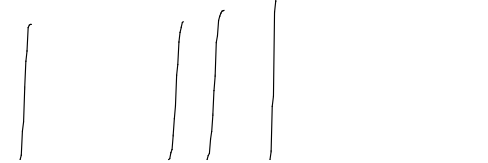

ळ

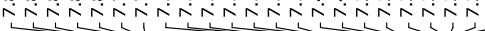

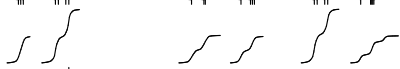
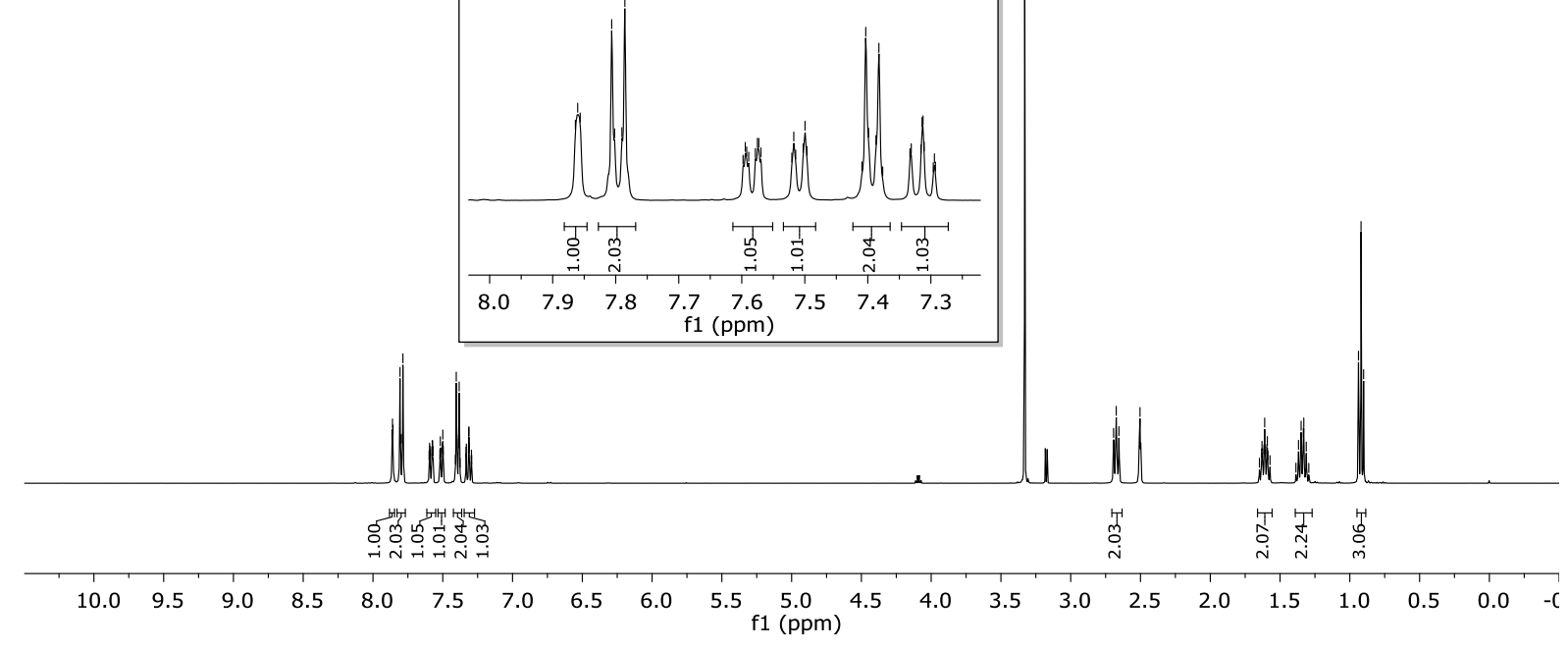

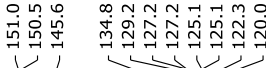

$\sum_{\substack{10 \\ 0}}^{\infty}$ ஸूल<smiles>[Mg]c1ccc(NNc2cccc([Se])c2)cc1</smiles>
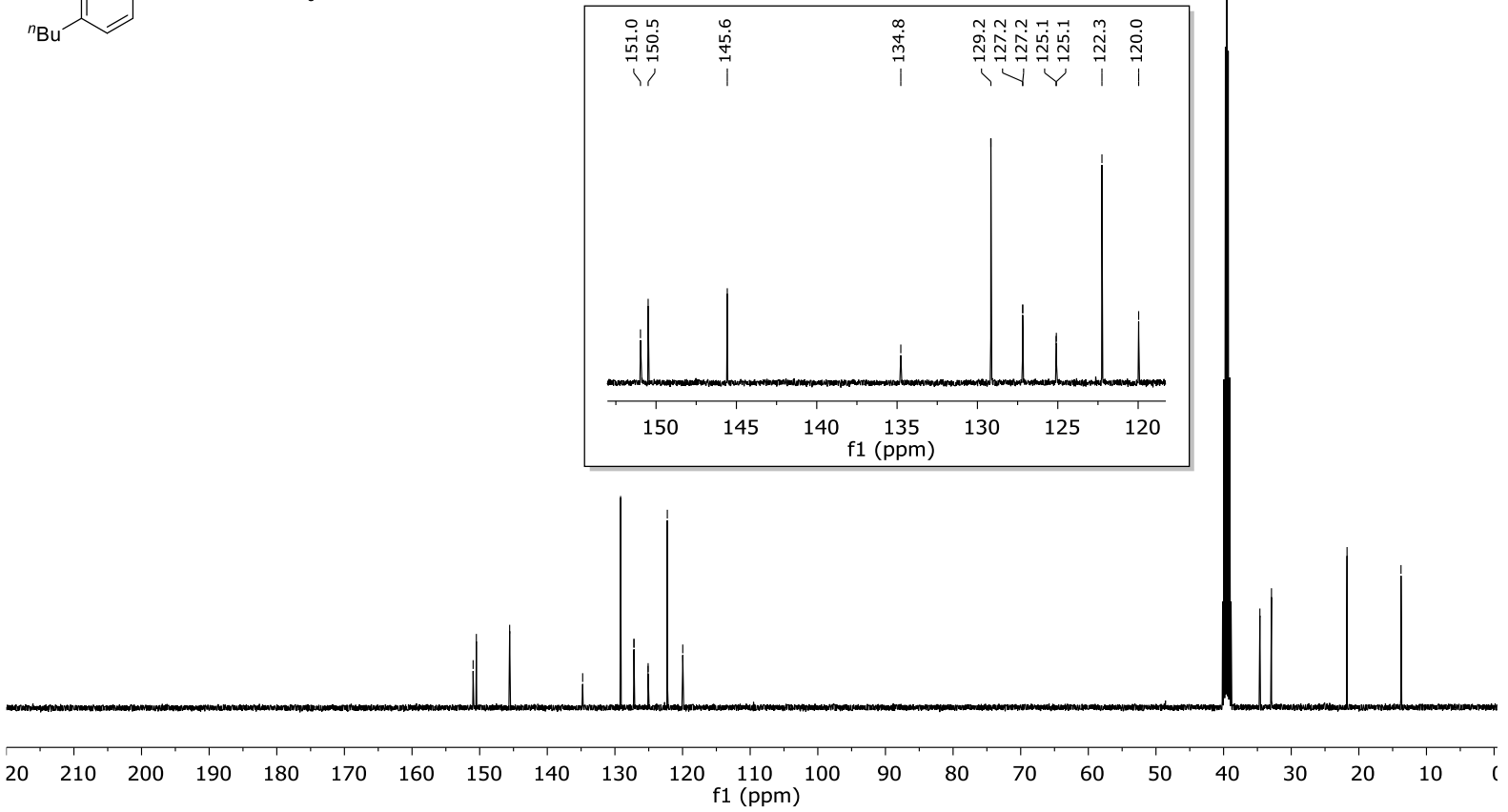


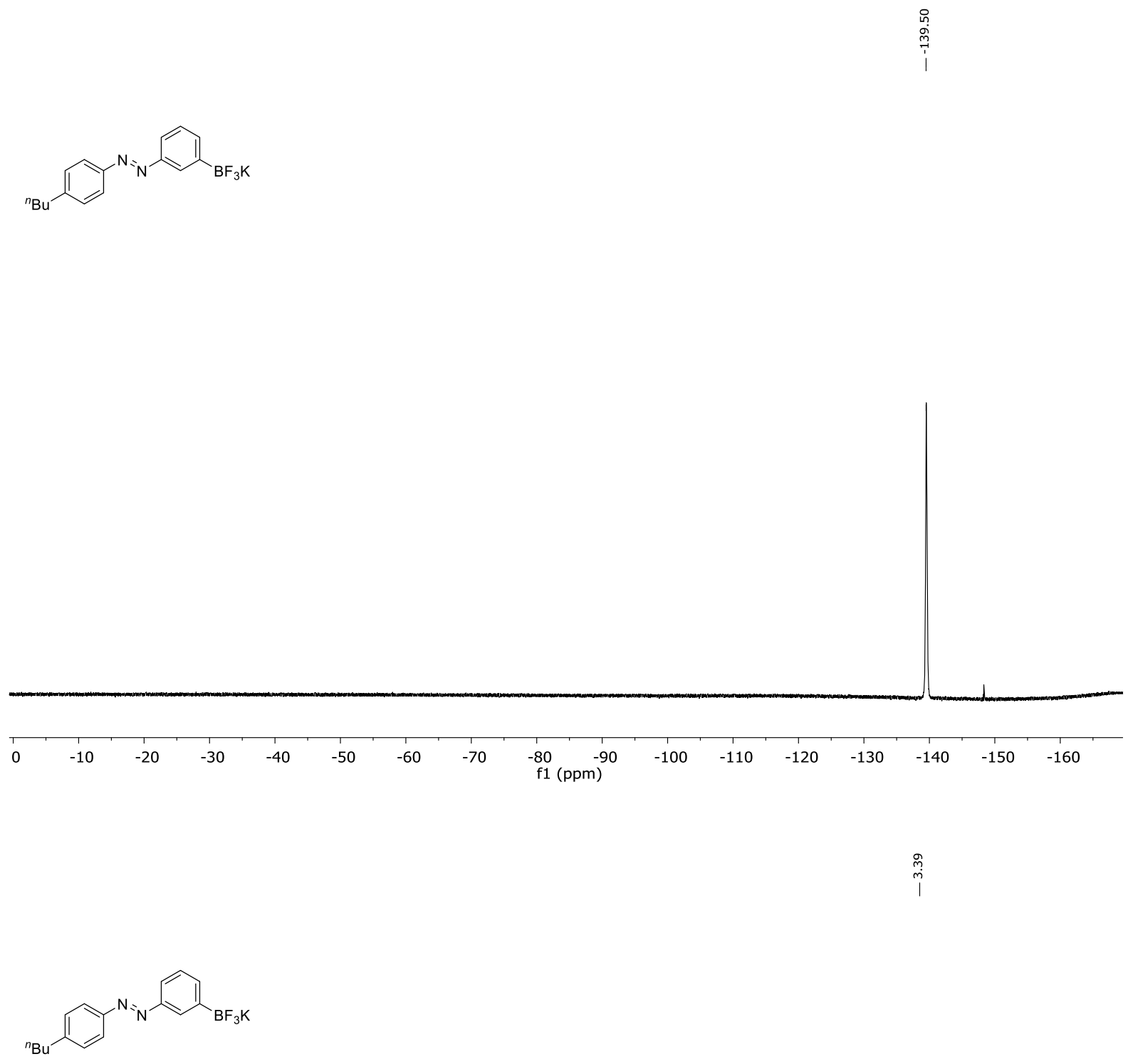




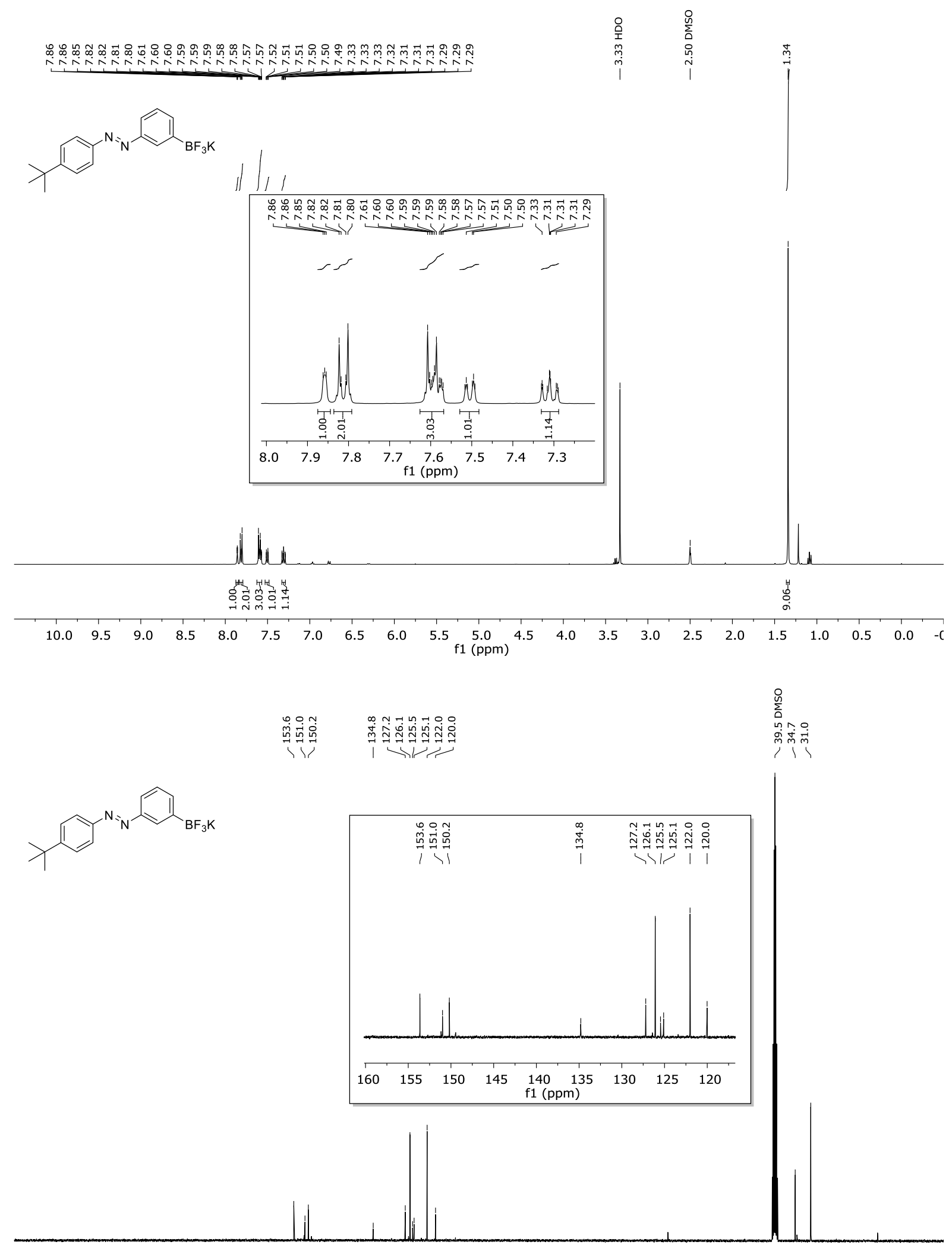

$\begin{array}{llllllllllllllllllllllll}20 & 210 & 200 & 190 & 180 & 170 & 160 & 150 & 140 & 130 & 120 & \begin{array}{l}110 \\ \mathrm{f} 1(\mathrm{ppm})\end{array} & 100 & 90 & 80 & 70 & 60 & 50 & 40 & 30 & 20 & 10 & (\end{array}$ 
${ }_{\mathrm{BF}_{3} \mathrm{~K}}^{\mathrm{N}}$

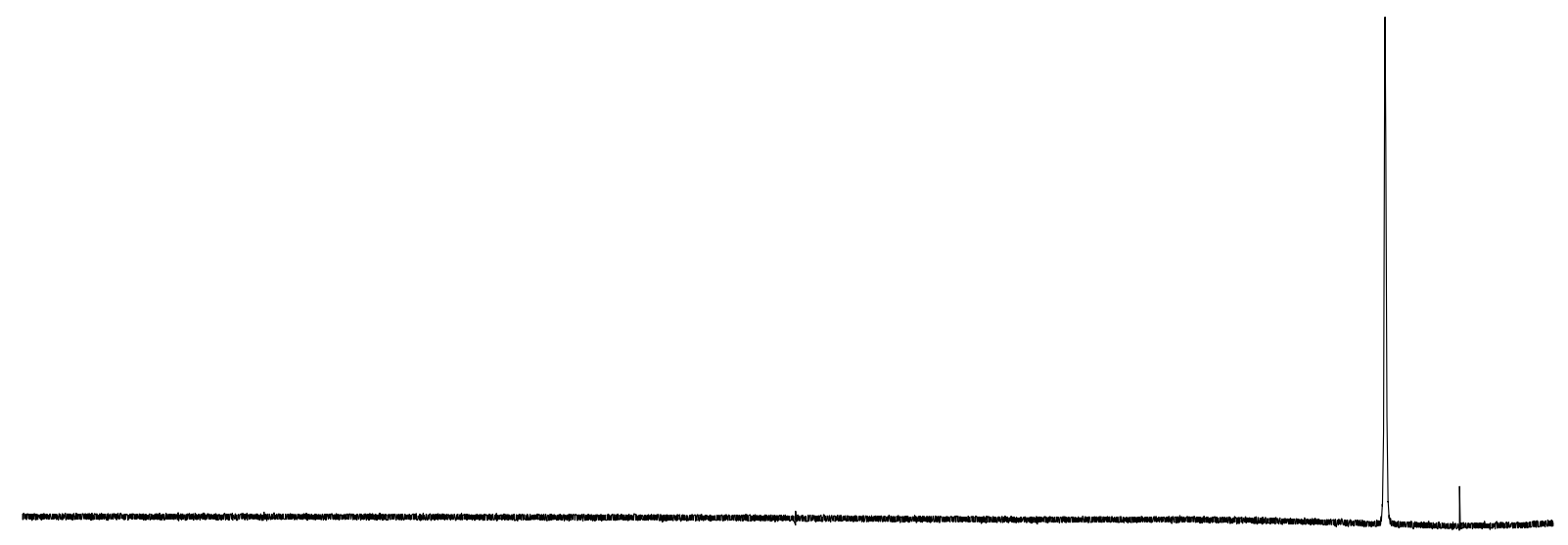

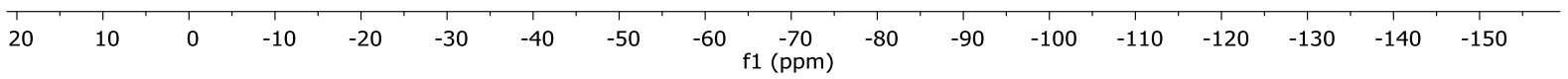

$\overbrace{\mathrm{BF}_{3} \mathrm{~K}}^{\mathrm{N}}$

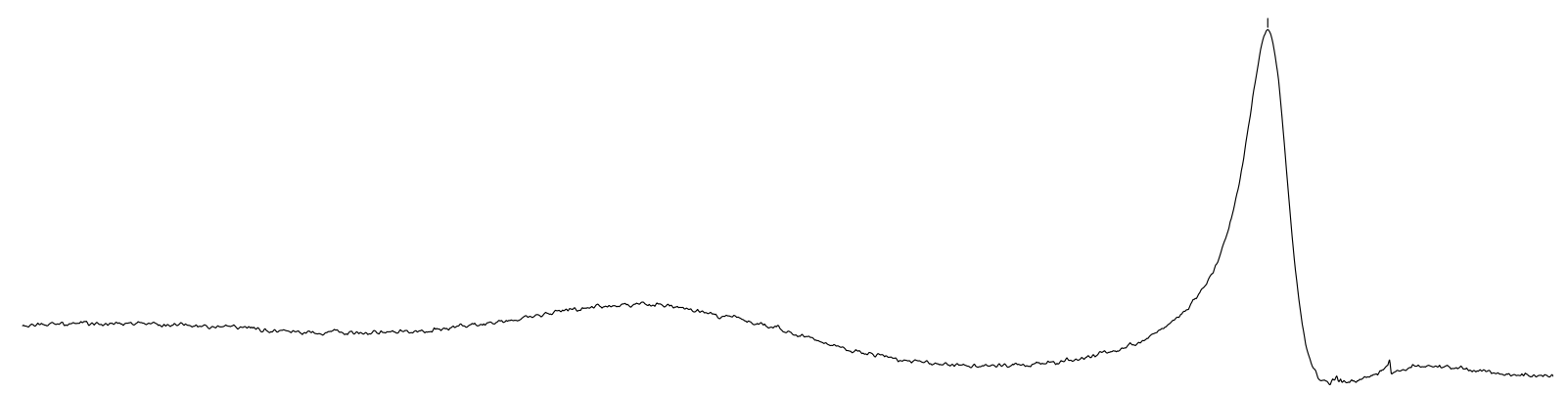

$\begin{array}{rllllllllllllllllllllllllllllllllllllll}50 & 48 & 46 & 44 & 42 & 40 & 38 & 36 & 34 & 32 & 30 & 28 & 26 & 24 & 22 & 20 & 18 & 16 & 14 & 12 & 10 & 8 & 6 & 4 & 2 & 0 & -2 & -4 & -6\end{array}$ 
(E)-4-((3-(trifluoro-14-boraneyl)phenyl)diazenyl)benzoic acid, potassium salt (14)
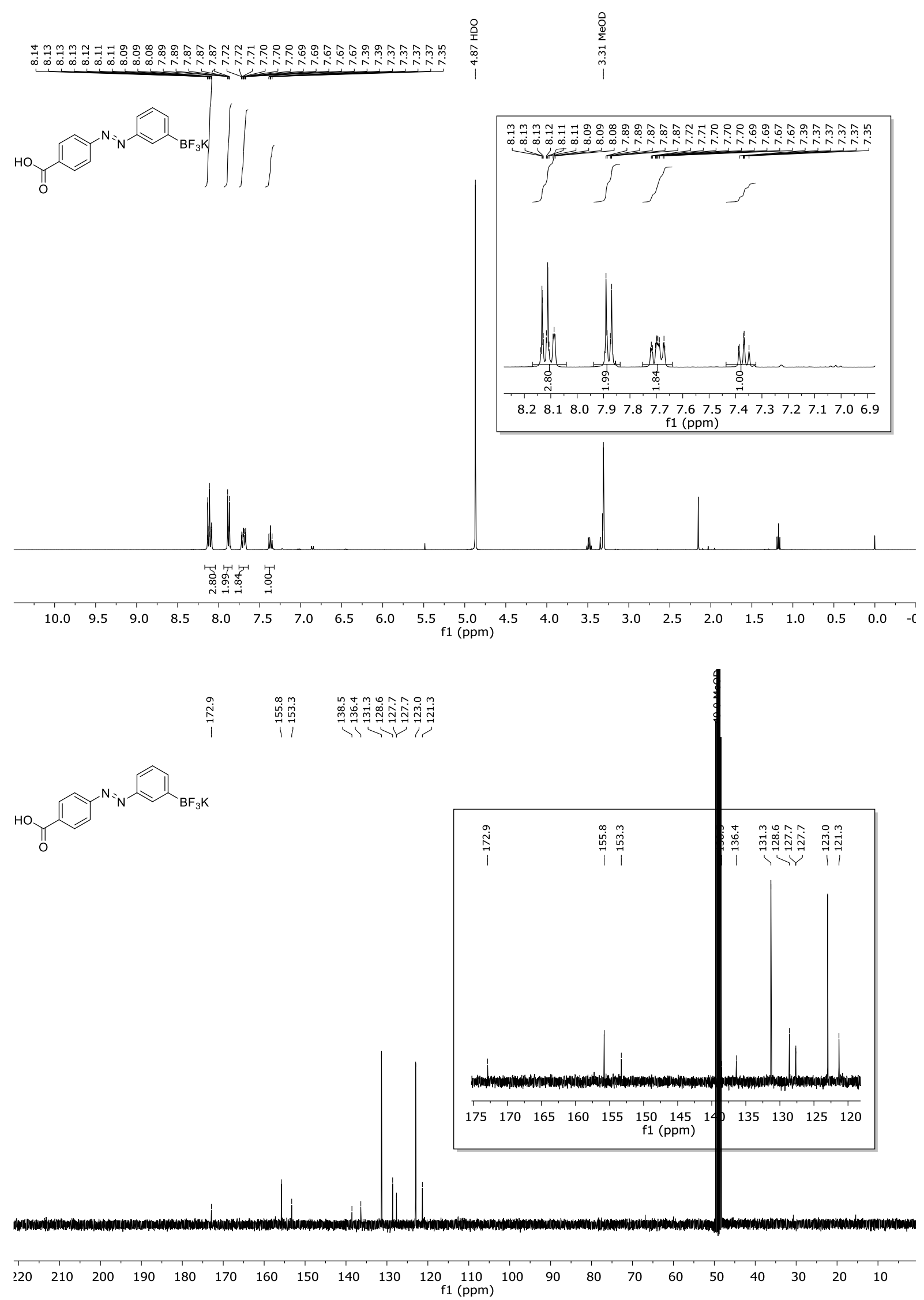
i
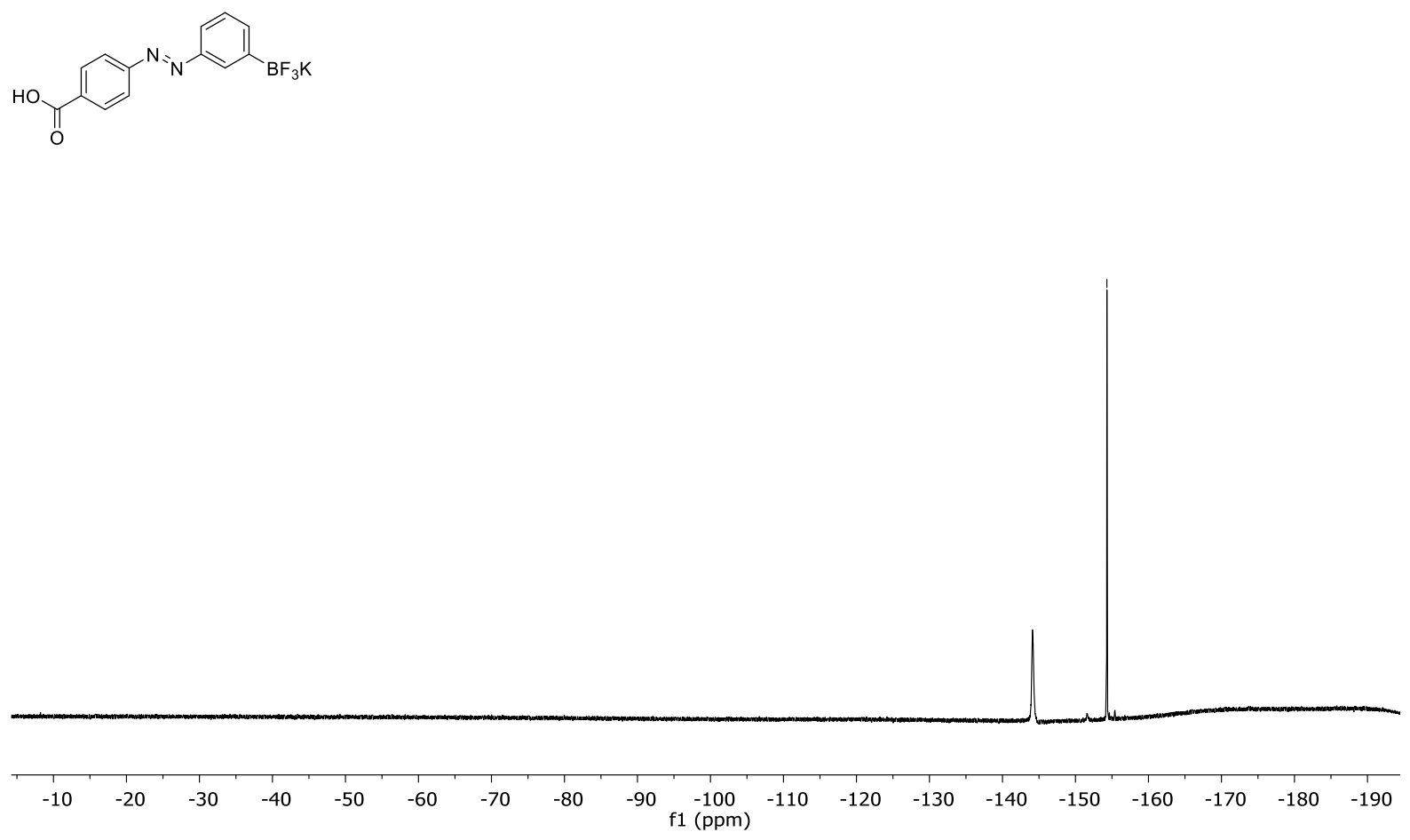

ִָ
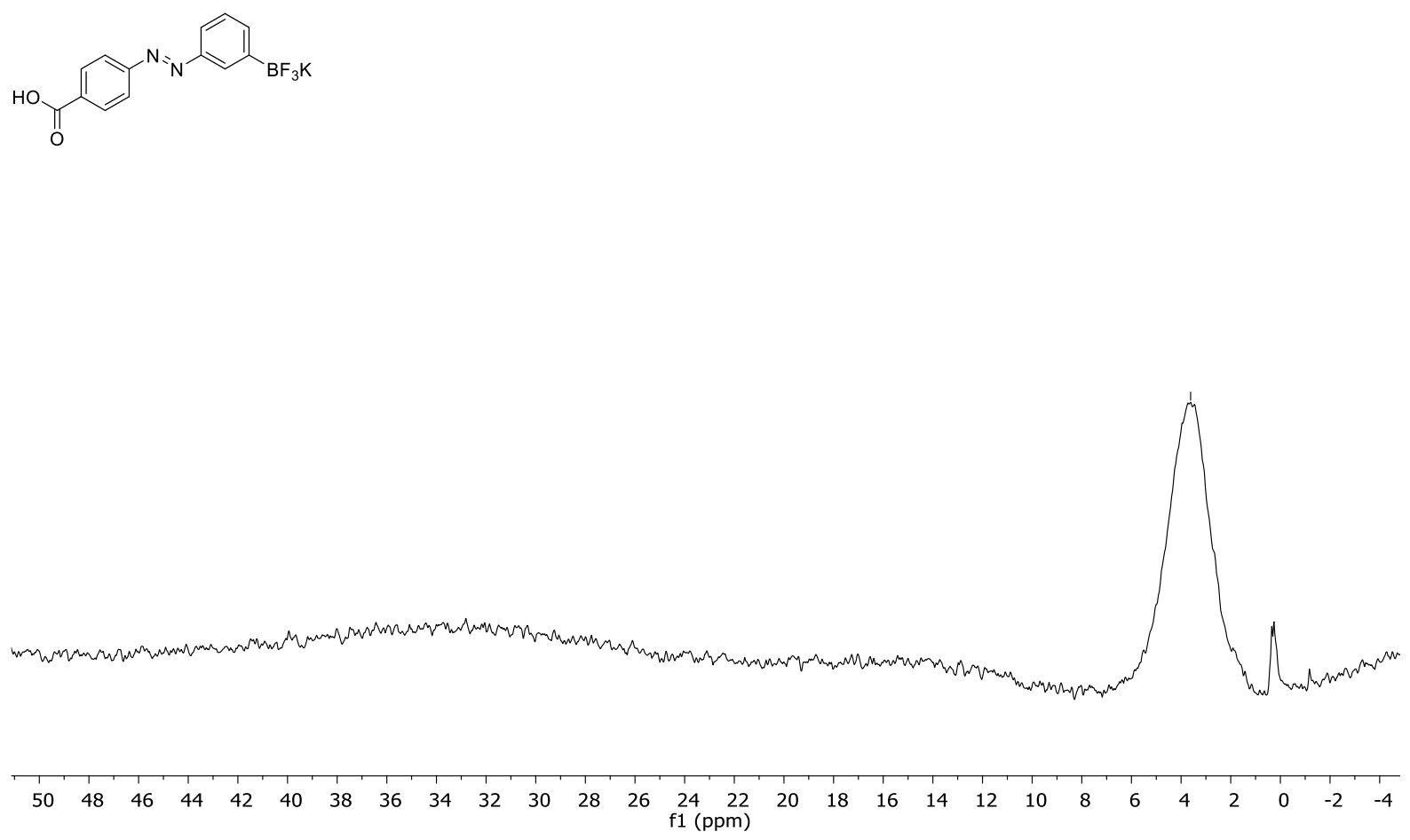

- S140 - 
Methyl (E)-4-((3-(trifluoro-l4-boraneyl)phenyl)diazenyl)benzoate, potassium salt (15)

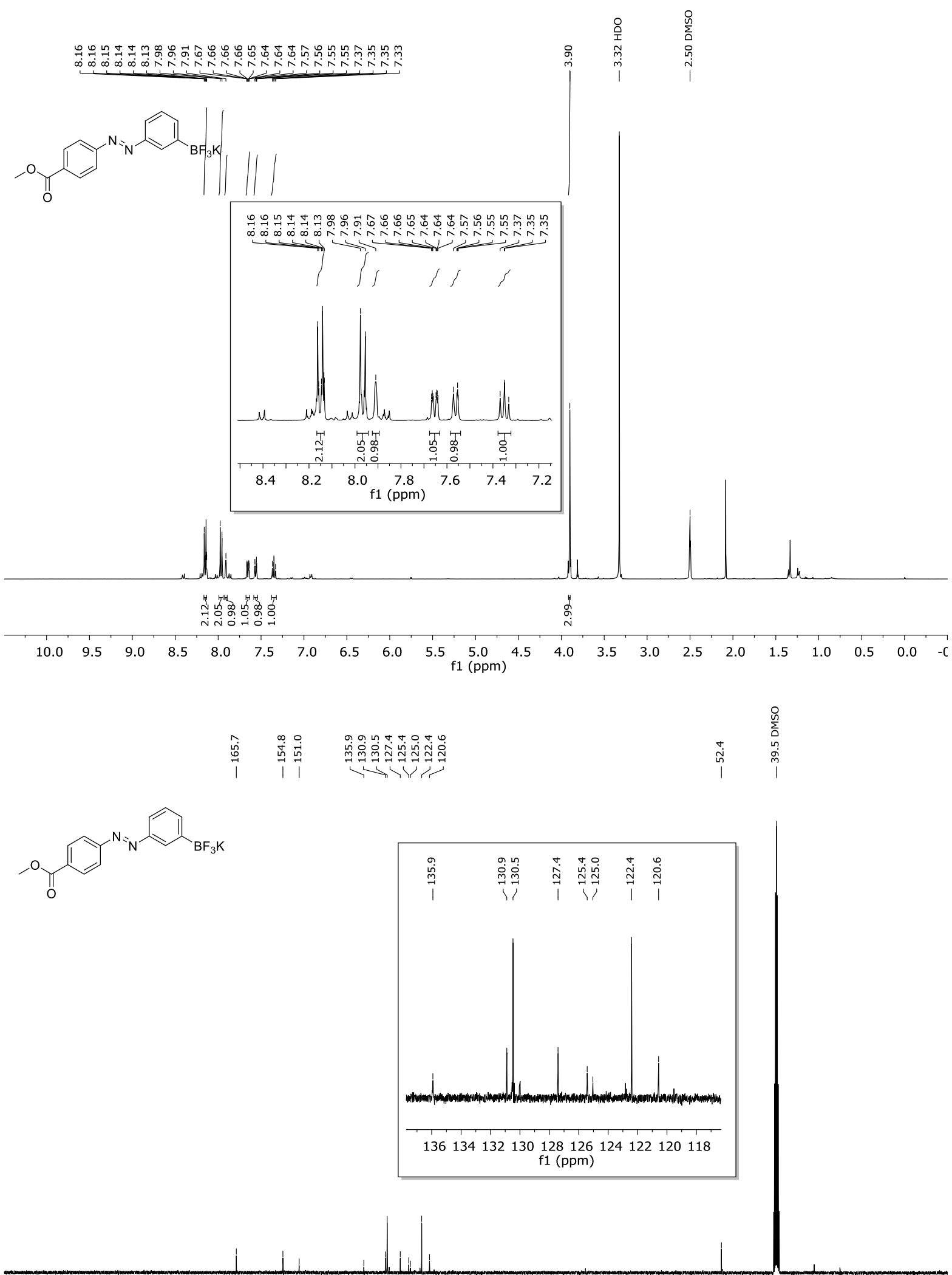

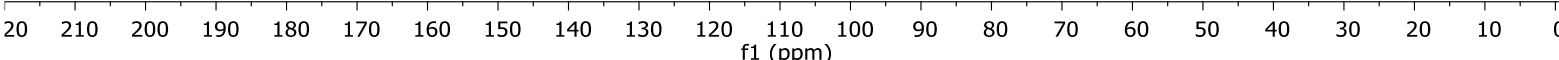


(n)

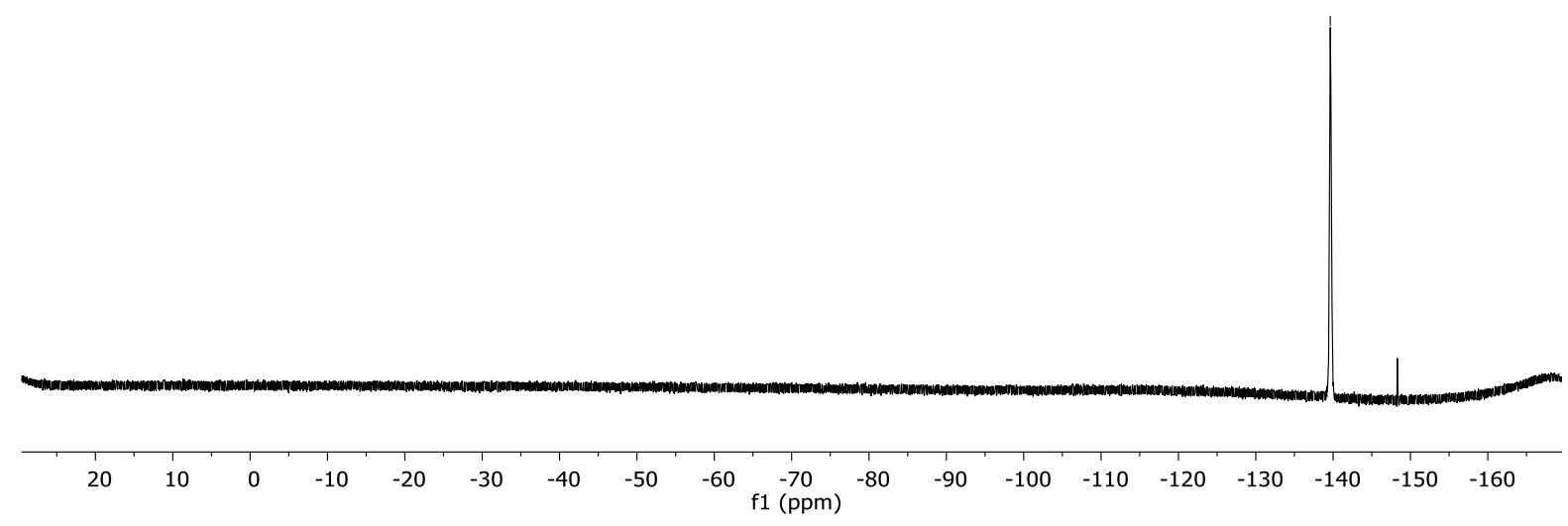

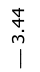<smiles>COC(=O)c1ccc(N=Nc2cccc([13CH]=[W])c2)cc1</smiles>

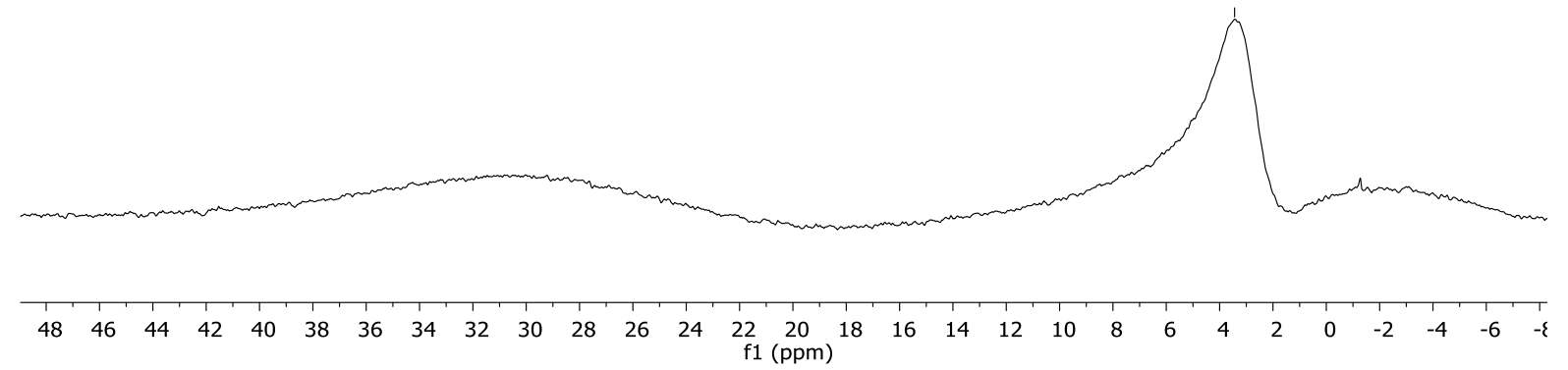



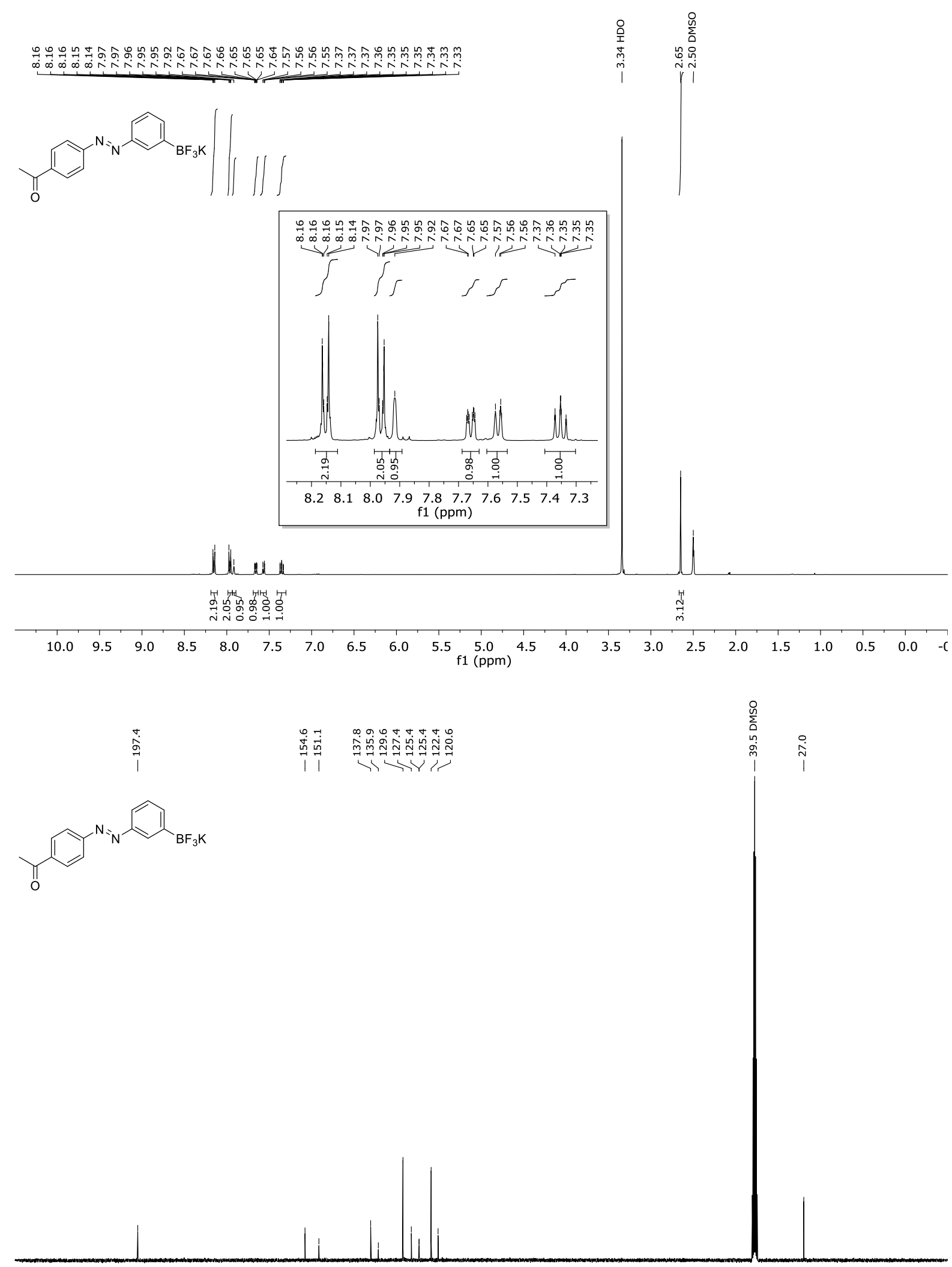

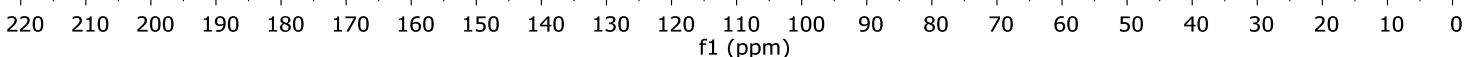


<smiles>CC(=O)c1ccc(NNc2cccc(Br)c2)cc1</smiles>
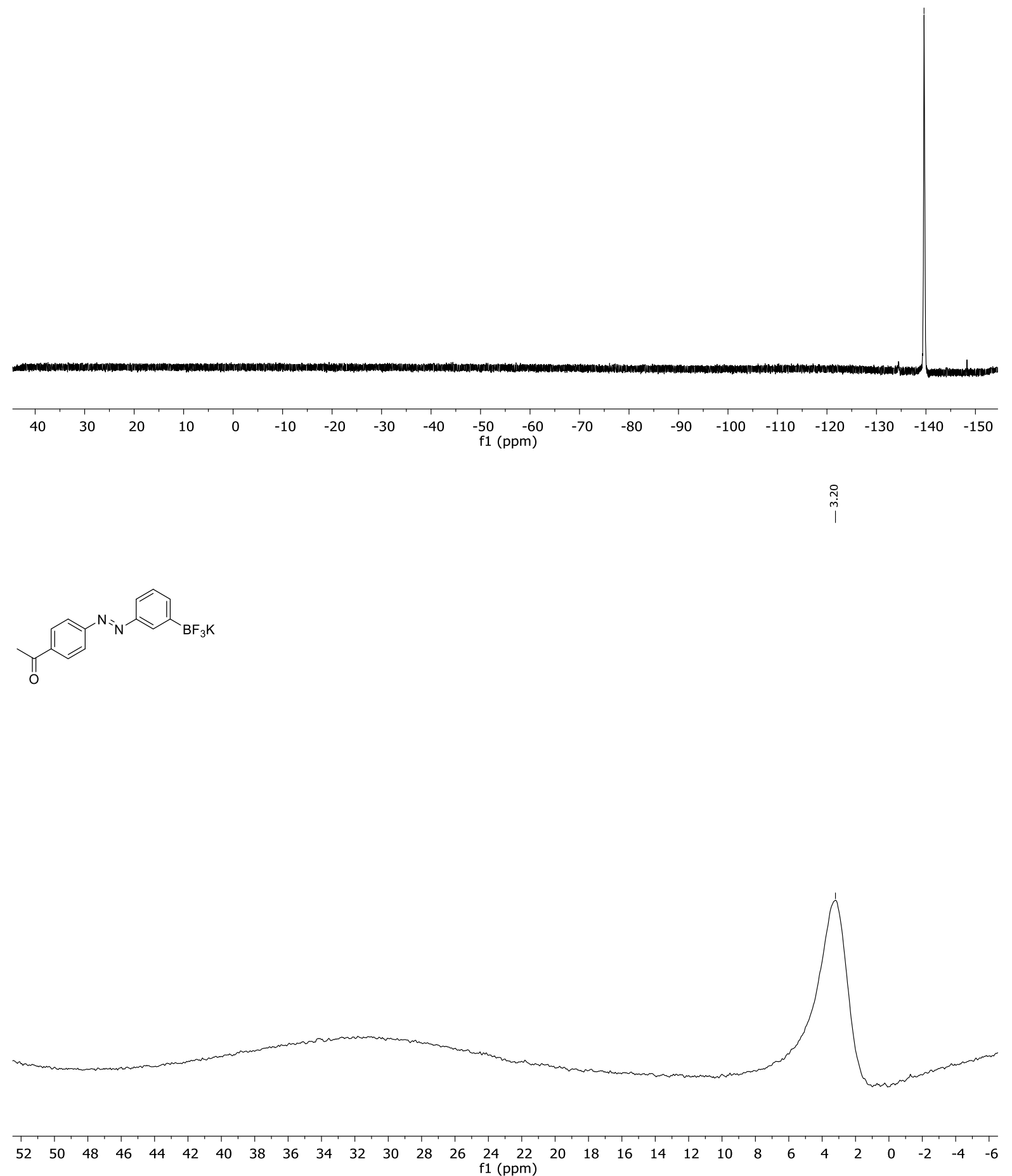

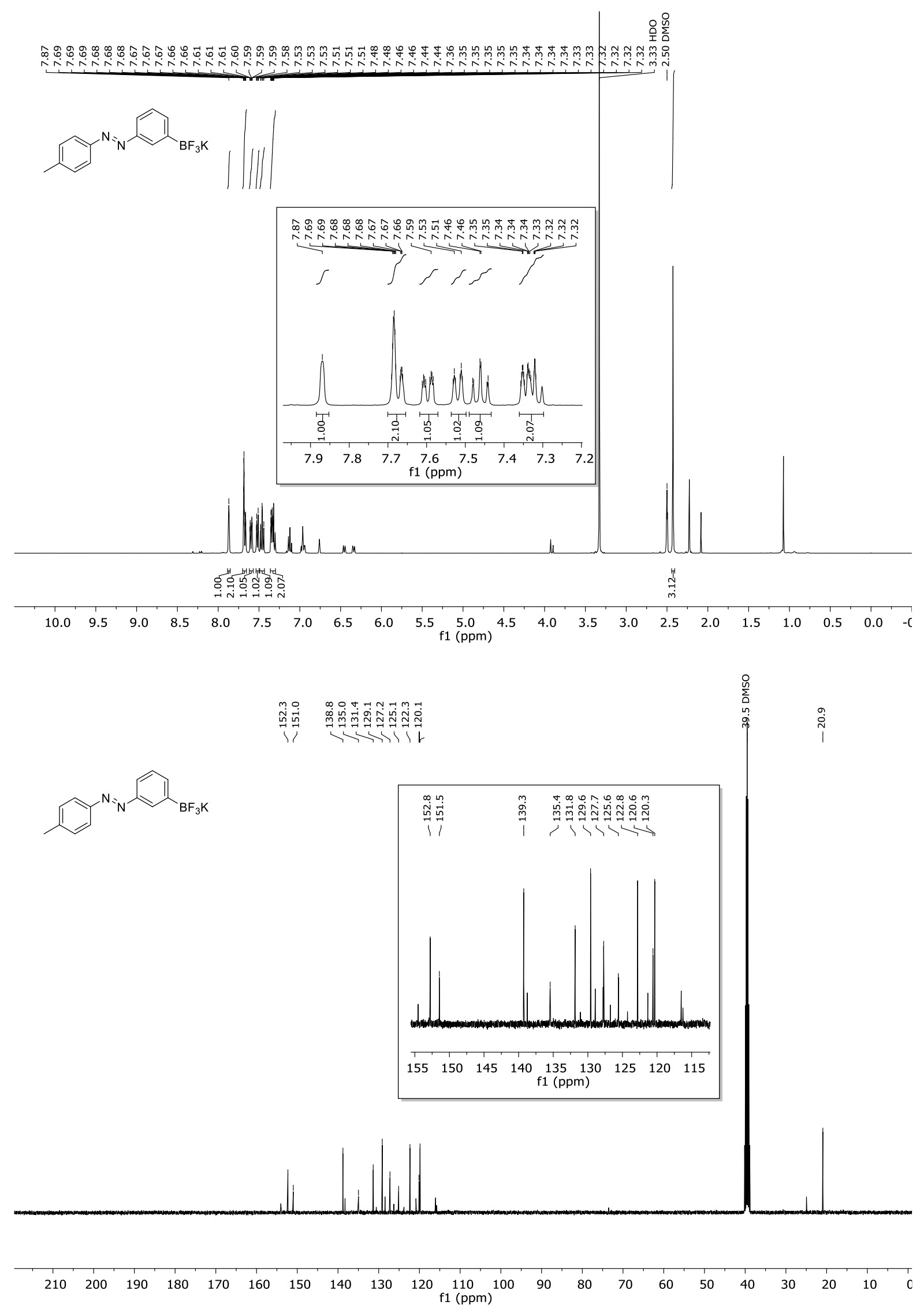
$\overbrace{\mathrm{N}}^{\mathrm{N}}$

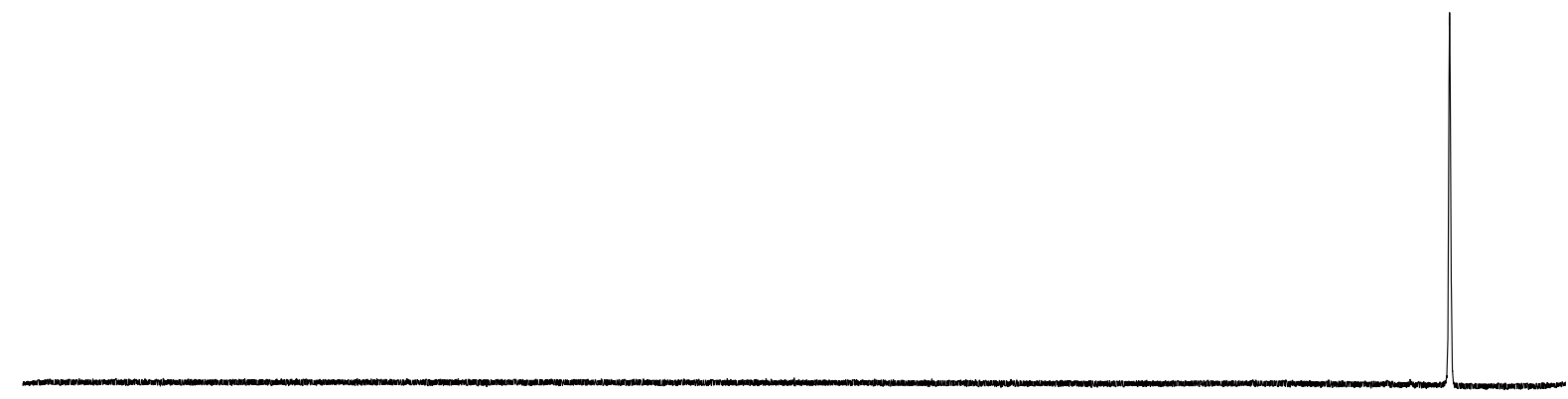

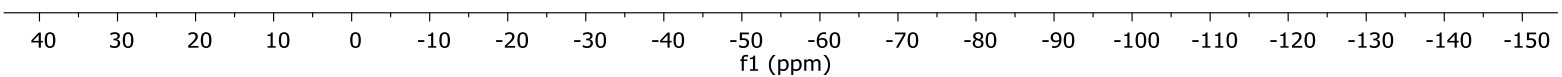

Nin

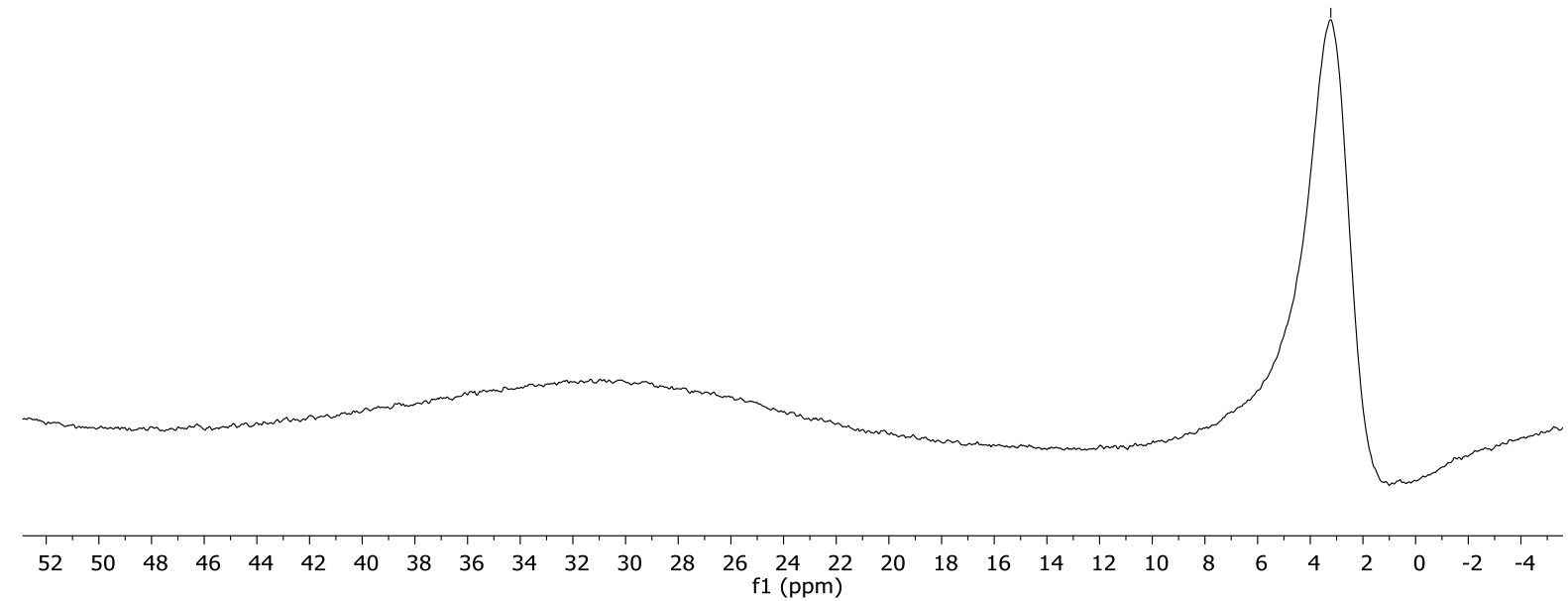


(E)-1-(2-methyl-3-(trifluoro-14-boraneyl)phenyl)-2-phenyldiazene, potassium salt (18)

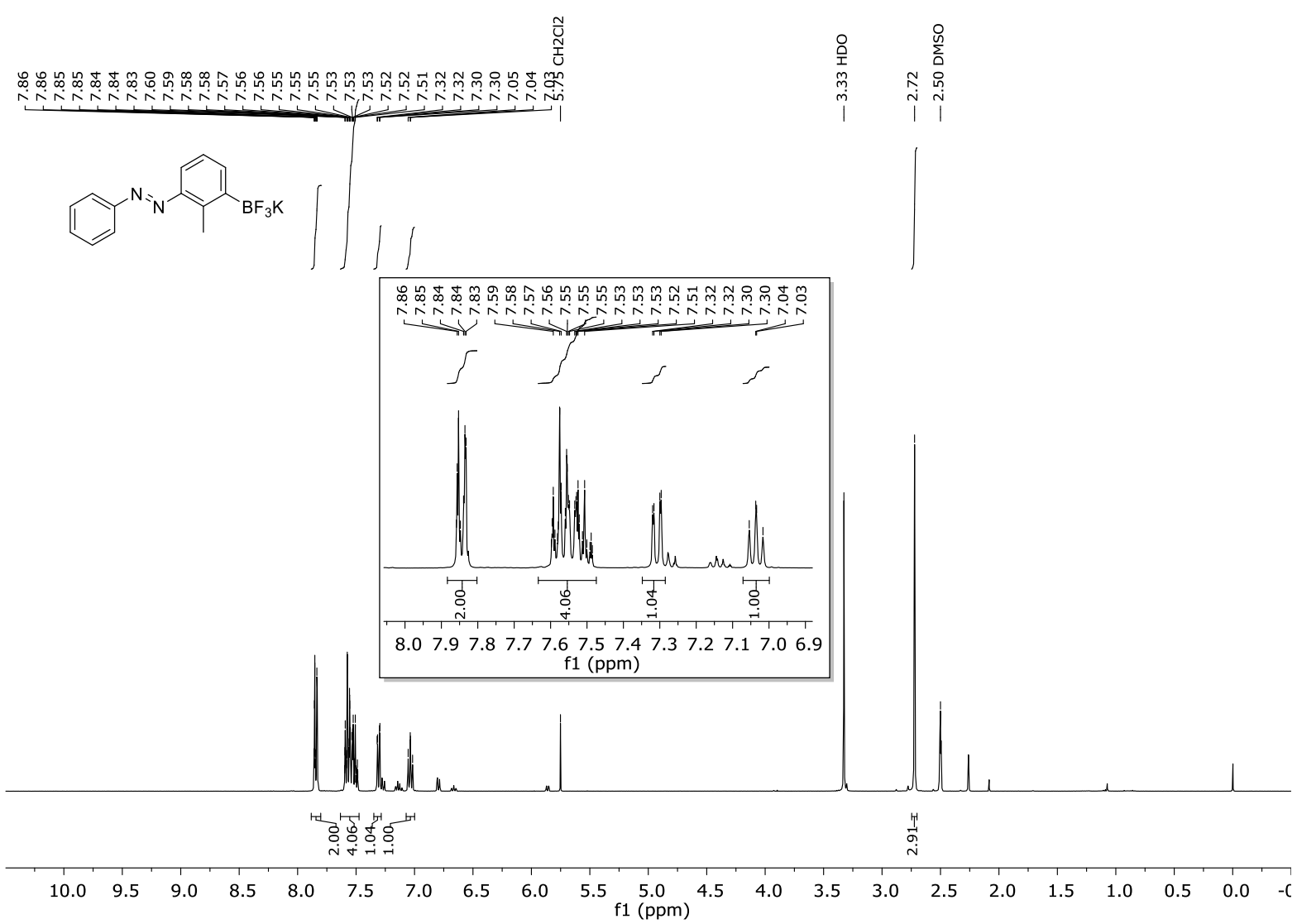

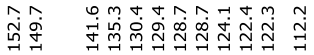
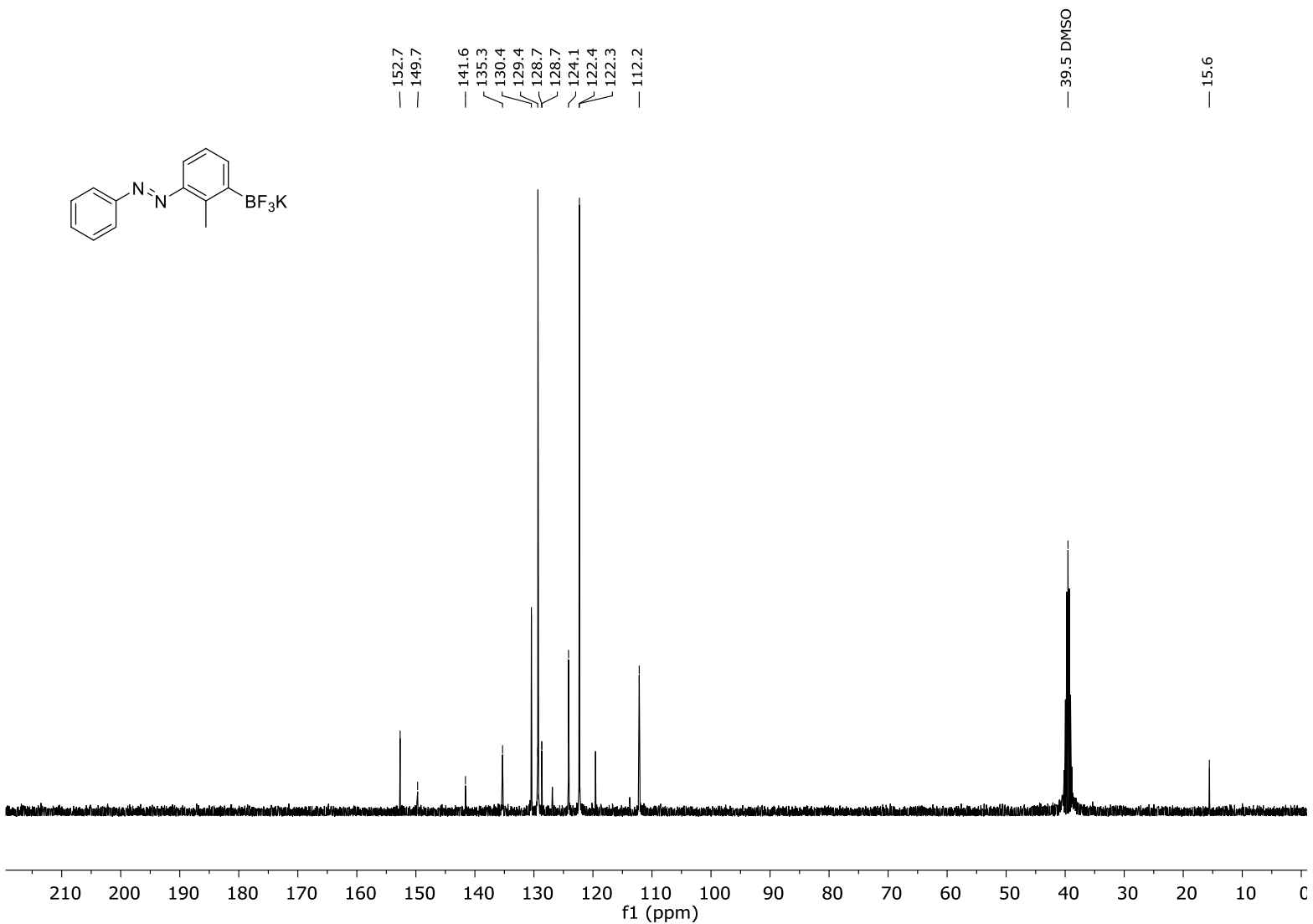


$$
\mathrm{N}^{\mathrm{N}{ }_{\mathrm{N}}}
$$
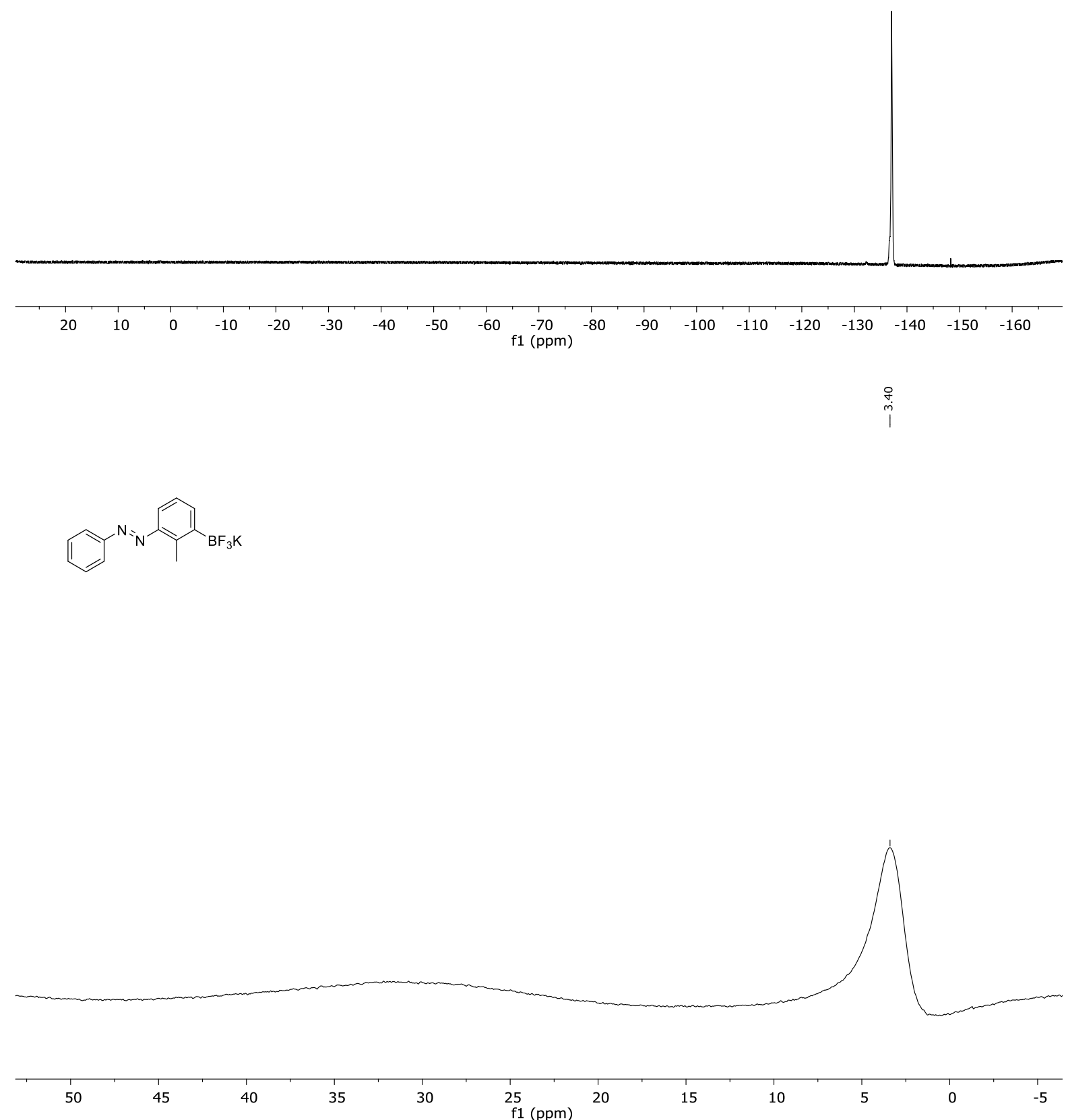


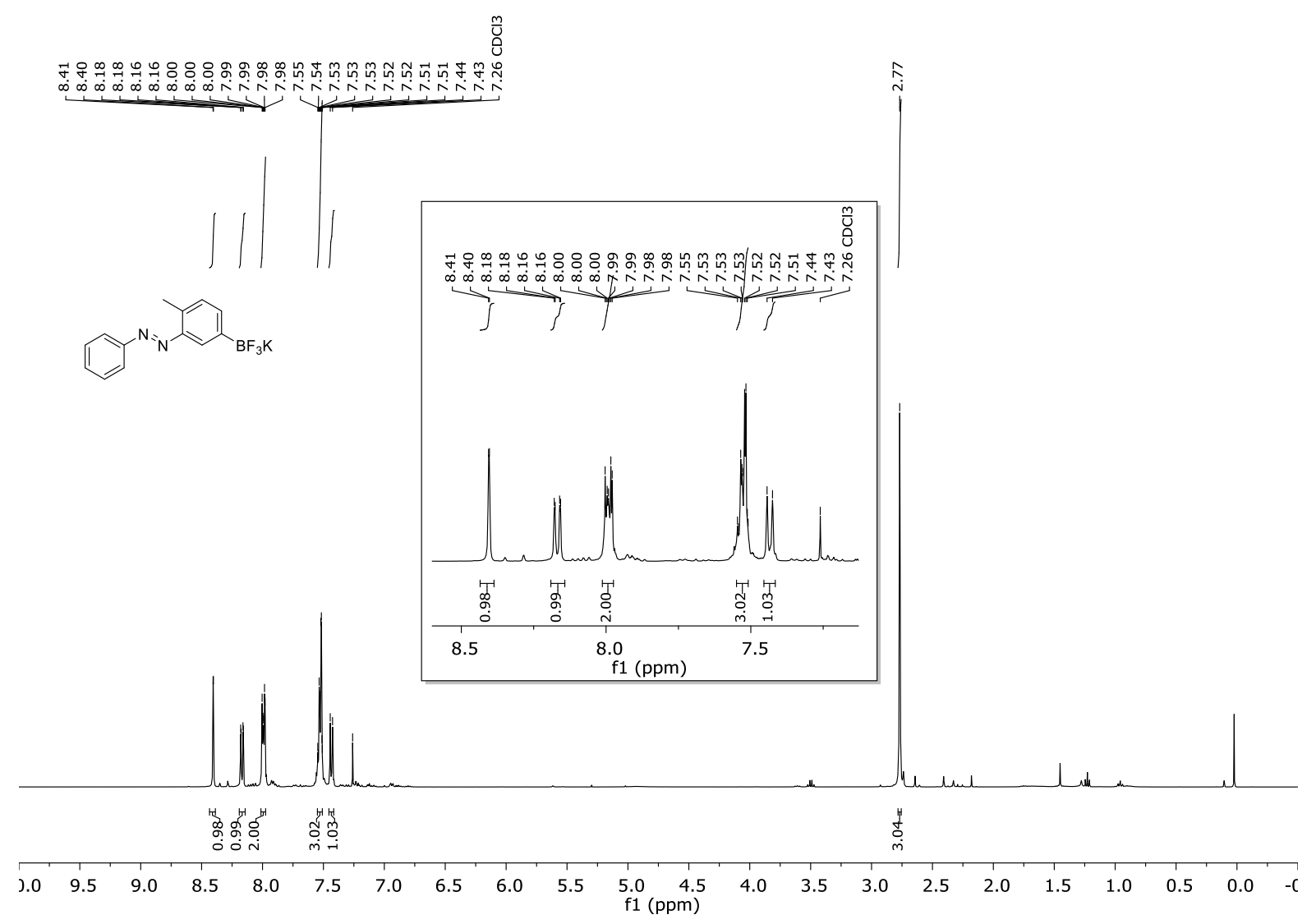

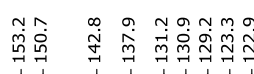

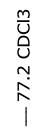

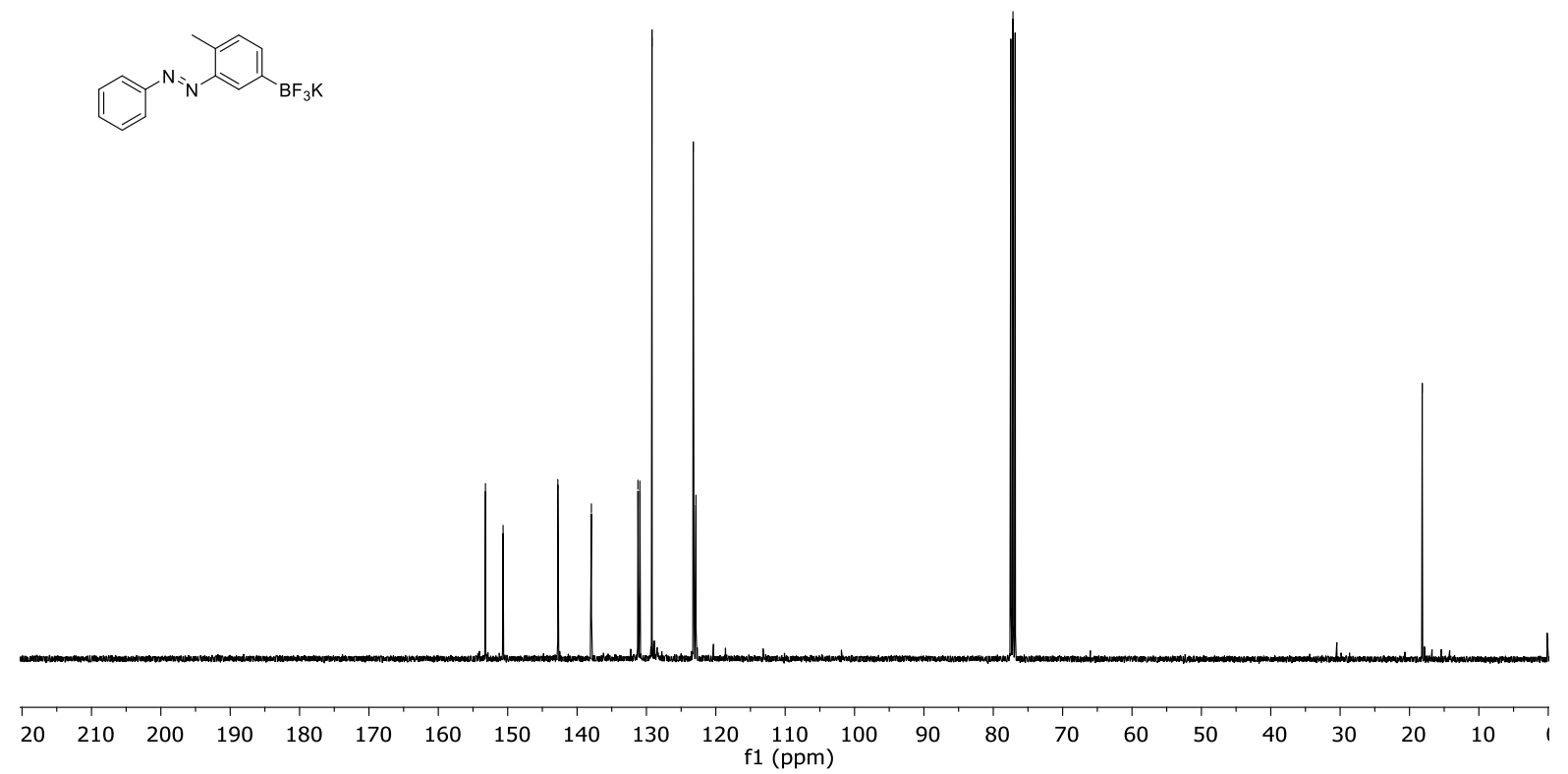


$\mathrm{N}^{\mathrm{N}_{\mathrm{N}}}$

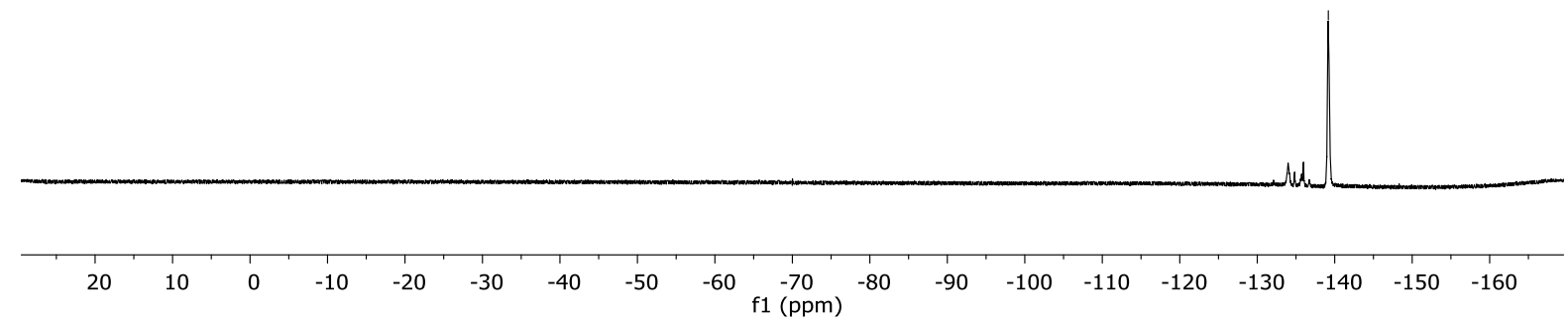

$\mathrm{y}^{\mathrm{N}_{\mathrm{N}}} \mathrm{Cl}_{\mathrm{BF}_{3} \mathrm{~K}}$

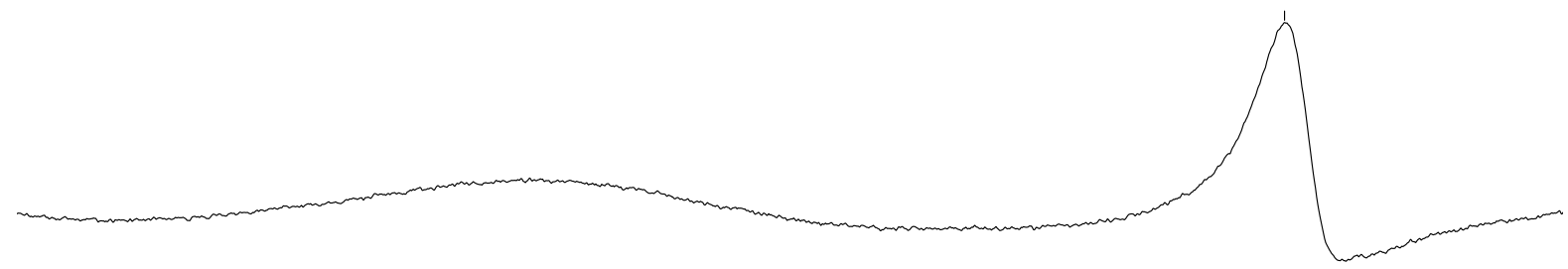

$\begin{array}{lllllllllllllllllllllllllllllllllllllllllllllll}50 & 48 & 46 & 44 & 42 & 40 & 38 & 36 & 34 & 32 & 30 & 28 & 26 & 24 & 22 & 20 & 18 & 16 & 14 & 12 & 10 & 8 & 6 & 4 & 2 & 0 & -2 & -4 & -6\end{array}$ 
(E)-1-(4-methyl-3-(trifluoro-14-boraneyl)phenyl)-2-phenyldiazene, potassium salt (20)

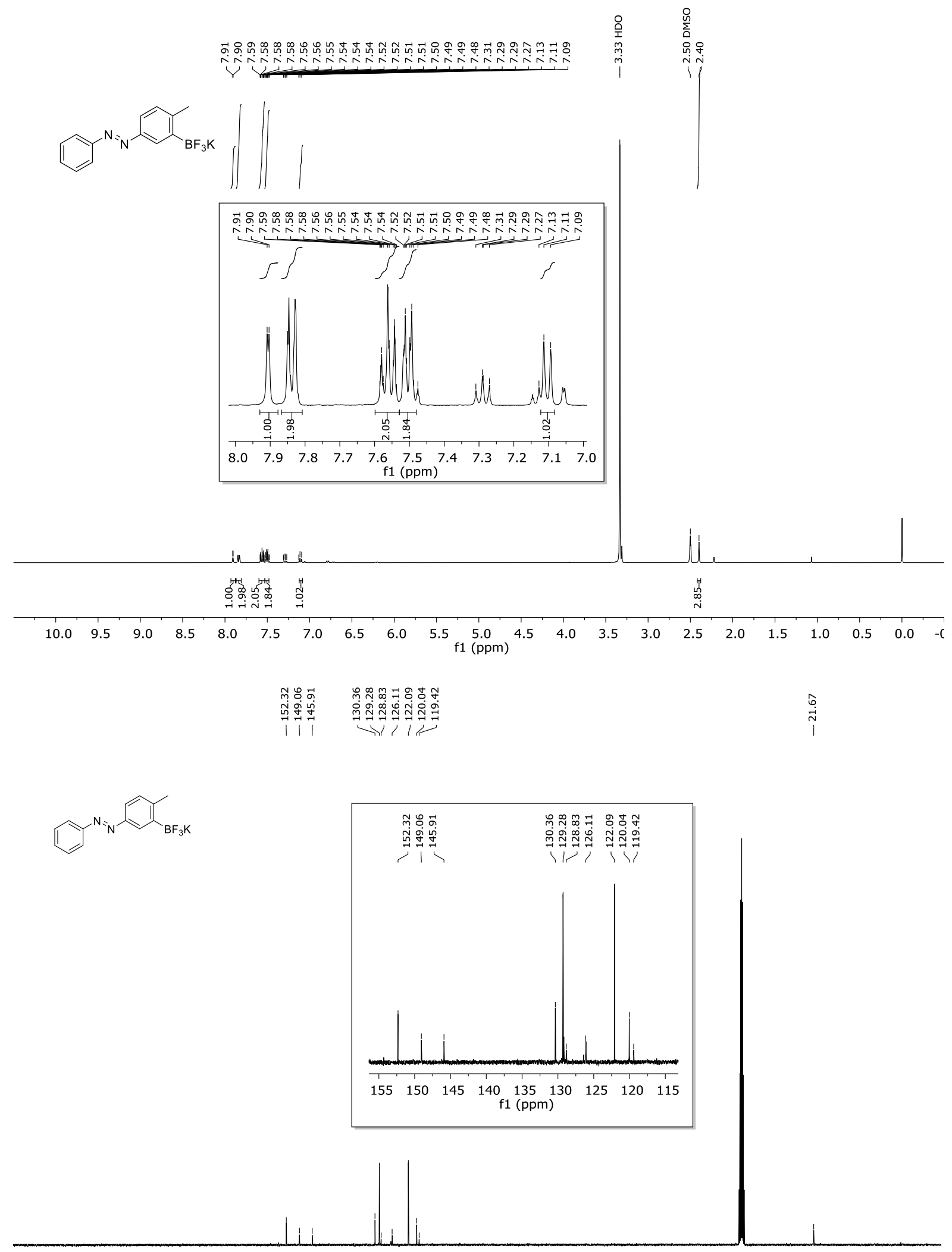

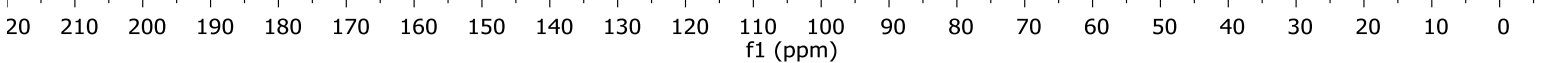


$\mathrm{Cr}_{\mathrm{N}}^{\mathrm{N}} \mathrm{BF}_{3 \mathrm{~K}}$
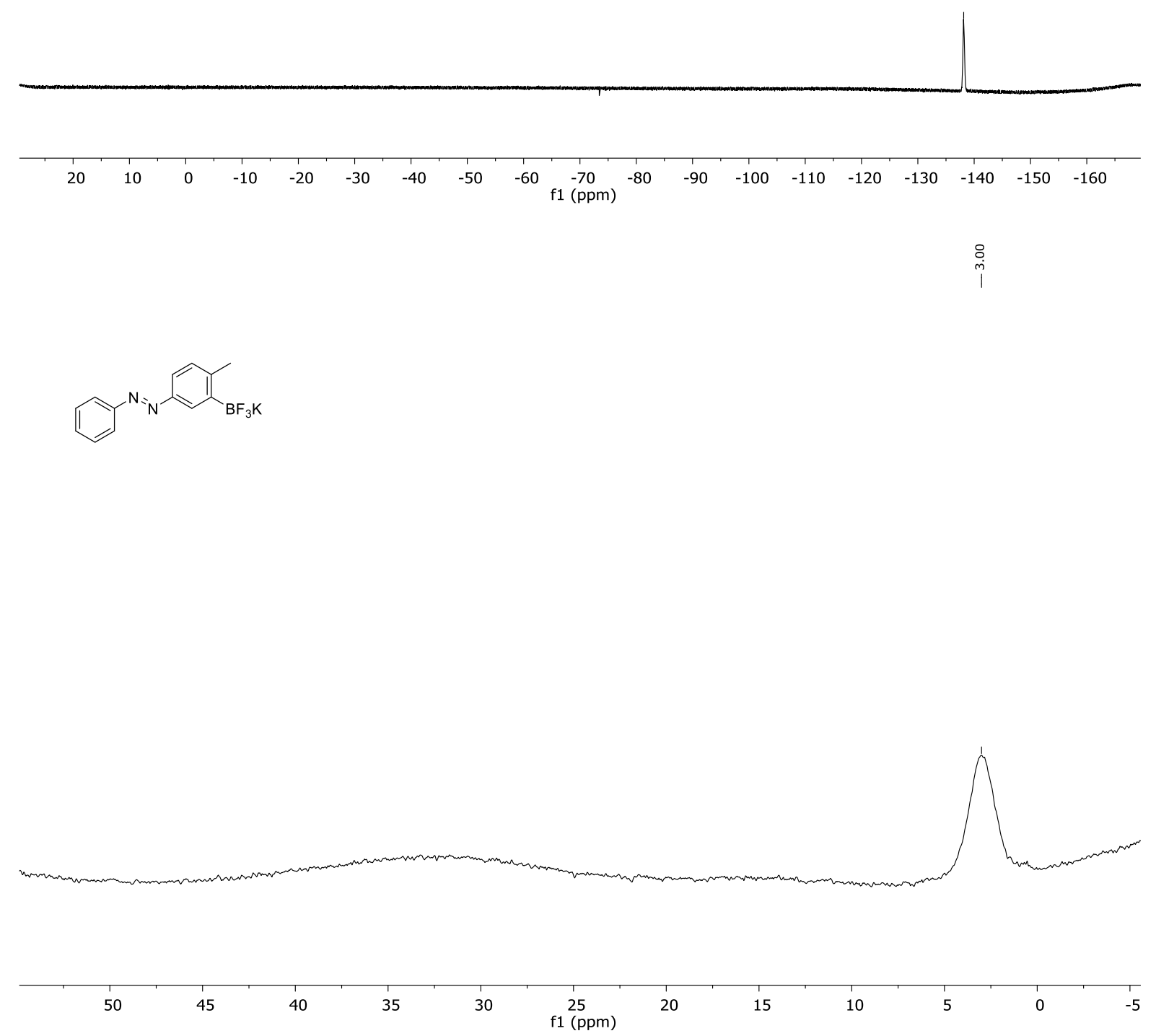
(E)-1-(4-methyl-3-(trifluoro-14-boraneyl)phenyl)-2-(p-tolyl)diazene, potassium salt (21)
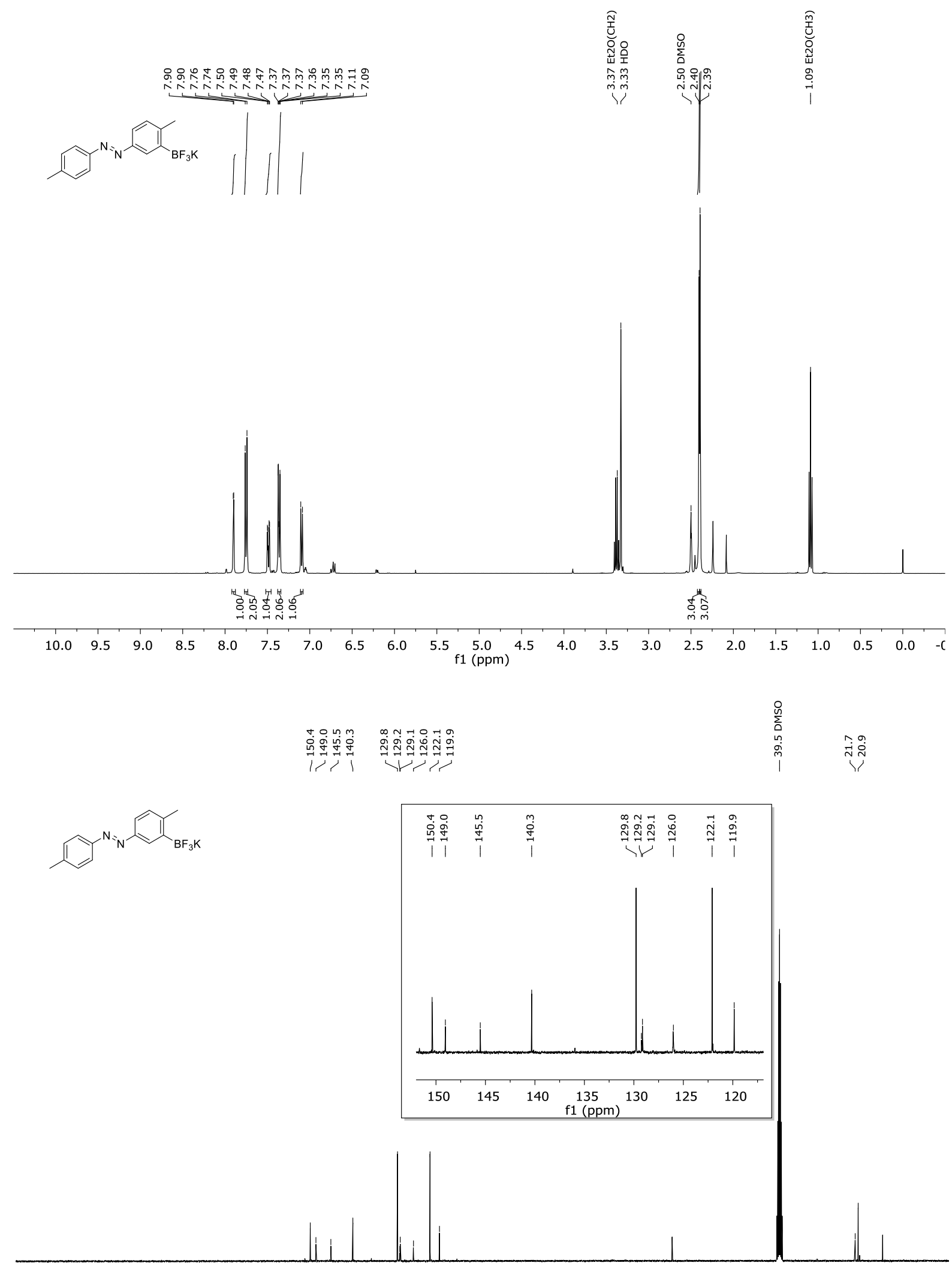

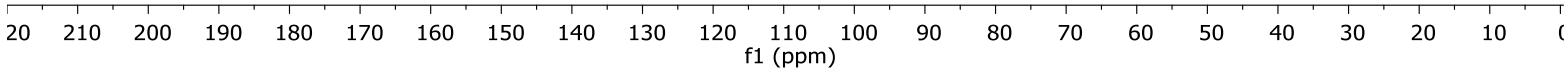




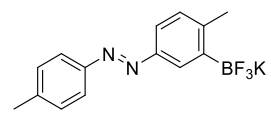

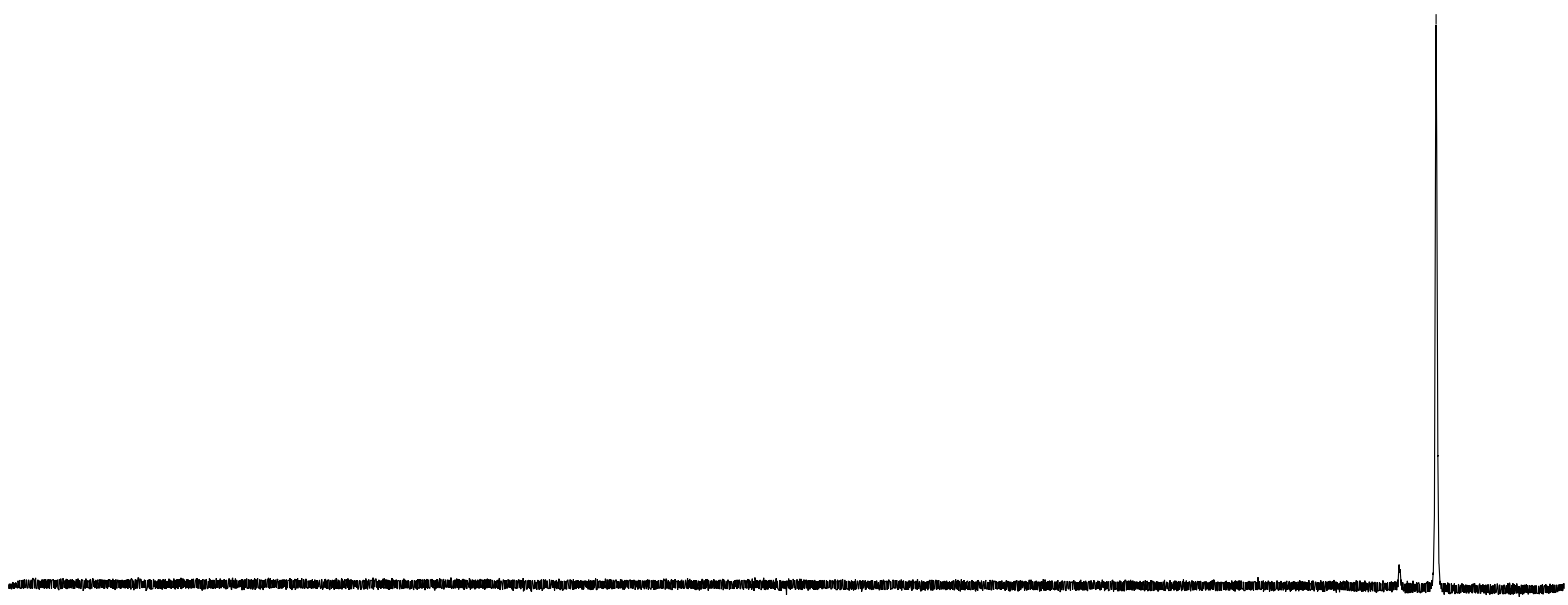

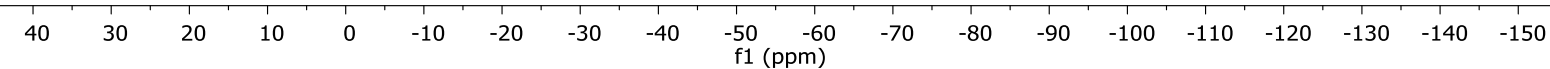

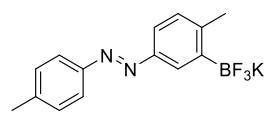

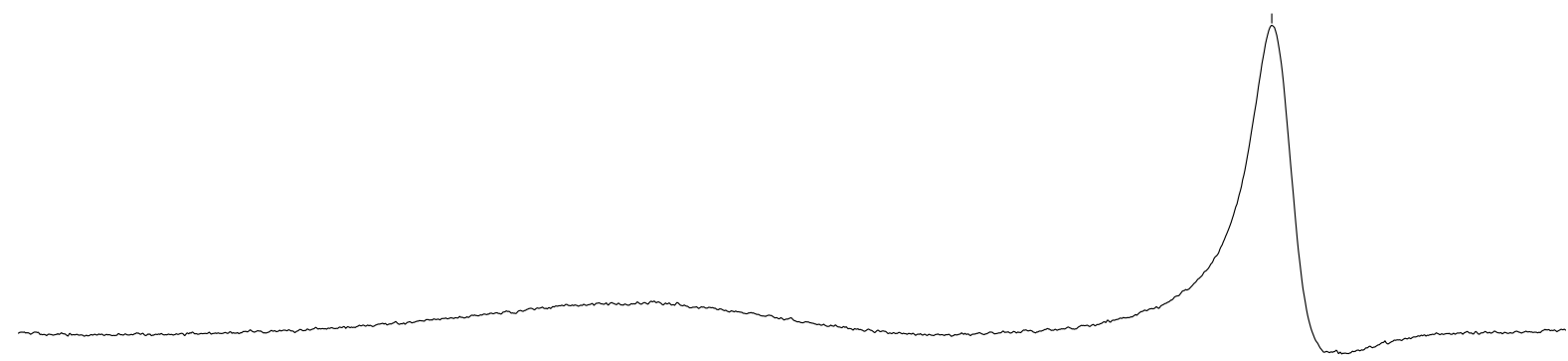

$\begin{array}{llllllllllllllllllllllllllllllllllll}54 & 52 & 50 & 48 & 46 & 44 & 42 & 40 & 38 & 36 & 34 & 32 & 30 & 28 & 26 & 24 & 22 & 20 & 18 & 16 & 14 & 12 & 10 & 8 & 6 & 4 & 2 & 0 & -2 & -4 & -6 & -8\end{array}$ 